\title{
Nonlinear THz spectroscopy on n-type GaAs
}

\author{
DISSERTATION \\ zur Erlangung des akademischen Grades \\ doctor rerum naturalium \\ (Dr. rer. nat.) \\ im Fach Physik \\ eingereicht an der \\ Mathematisch-Naturwissenschaftlichen Fakultät I \\ Humboldt-Universität zu Berlin \\ von \\ Herr Dipl.-Ing. Peter Gaál \\ geboren am 26.04.1976 in Bratislava
}

Präsident der Humboldt-Universität zu Berlin:

Prof. Dr. Dr. h.c. Christoph Markschies

Dekan der Mathematisch-Naturwissenschaftlichen Fakultät I:

Prof. Dr. Lutz-Helmut Schön

Gutachter:

1. Prof. Dr. Thomas Elsässer

2. Prof. Dr. Oliver Benson

3. Univ. Prof. Dr. Karl Unterrainer

eingereicht am:

25.04 .2008

Tag der mündlichen Prüfung: $\quad$ 23.06.2008 


\begin{abstract}
In this thesis, the ultrafast dynamics of conduction band electrons in semiconductors are investigated by nonlinear terahertz ( $\mathrm{THz})$ spectroscopy. In particular, n-doped gallium arsenide samples with doping concentrations in the range of $10^{16} \mathrm{~cm}^{-3}$ to $10^{17} \mathrm{~cm}^{-3}$ are studied. A novel source for the generation of intense $\mathrm{THz}$ radiation is developed which yields single-cycle $\mathrm{THz}$ transients with field amplitudes of more then $400 \mathrm{kV} / \mathrm{cm}$. The $\mathrm{THz}$ source uses ultrashort optical laser pulses provided by a Ti:sapphire oscillator. In addition, a two-color THz-pump mid-infrared-probe setup is implemented, which allows for two-dimensional time-resolved experiments in the far-infrared wavelength range.

Field ionization of neutral shallow donors in gallium arsenide with intense, ultrashort $\mathrm{THz}$ pulses and subsequent coherent radiative recombination of electrons to impurity ground states is observed at room temperature. The superradiant decay of the nonlinear polarization results in the emission of a coherent signal with picosecond lifetimes. Such nonlinear signals, which exhibit a lifetime ten times longer than in the linear regime are observed for the first time. At low temperatures and $\mathrm{THz}$ field strengths below $5 \mathrm{kV} / \mathrm{cm}$, Rabi flopping on shallow donor transitions is demonstrated.

For the first time, the polar electron-LO phonon interaction is directly measured in the quantum kinetic transport regime. Quasi-instantaneous acceleration of conduction band electrons in the polar gallium arsenide lattice by the electric field of intense $\mathrm{THz}$ pulses and subsequent probing of the mid-infrared transmission reveals a modulation of the transmission along the THz-mid-infrared delay coordinate with the frequency of the LO phonon. These modulations directly display the relative phase between the electron motion and its surrounding virtual phonon cloud. Quantum kinetic model calculations fully account for the observed phenomena.
\end{abstract}

\title{
Keywords:
}

ultrafast spectroscopy, THz generation, gallium arsenide, Quantum kinetic charge transport,shallow donors 


\section{Zusammenfassung}

In dieser Arbeit wird die ultraschnelle Dynamik von Leitungsbandelektronen in Halbleitermaterialien mit Hilfe nichtlinearer Terahertz-Spektroskopie erforscht. Insbesondere wird n-dotiertes Galliumarsenid bei mittleren Dotierdichten zwischen $10^{16} \mathrm{~cm}^{-3}$ und $10^{17} \mathrm{~cm}^{-3}$ untersucht. Für die Erzeugung intensiever $\mathrm{THz}$ Strahlung wurde eine neuartige Quelle entwickelt, die $\mathrm{THz}$ Transienten mit nur einer Oszillationsperiode und maximalen Feldamplituden von mehr als $400 \mathrm{kV} / \mathrm{cm}$ liefert. Diese THz-Quelle benutzt ultrakurze optische Laserpulse aus einem Ti:Saphir Oszillator. Zusätzlich wurde ein neuartiger zwei-Farben Anrege-Abtast Experimentierplatz aufgebaut, der zweidimensionale, zeitaufgelöste Messungen im mittleren und fernen Infrarotbereich ermöglicht.

Feldionisation flacher, neutraler Störstellen im Galliumarsenid-Gitter mittels intensiver, ultrakurzer $\mathrm{THz}$ Impulse und die anschliessende kohärente, strahlende Rekombination von Elektronen in die Störstellen-Grundzustände bei Raumtemperatur wird gezeigt. Der superradiante Zerfall der nichtlinearen Polarisation führt zur Abstrahlung eines kohärenten Signals mit Lebensdauern von über einer Pikosekunde. Solche nichtlinearen Signale, die 10-fache Lebensdauern im Vergleich zum linearen Fall aufweisen, wurden in dieser Arbeit zum ersten Mal gemessen. Bei niedrigen Temperaturen und THz Feldstärken unter $5 \mathrm{kV} / \mathrm{cm}$ werden Rabi-Oszillationen an Übergängen in flachen Störstellen demonstriert.

Zum ersten Mal konnte die polare Elektron-LO-Phonon Wechselwirkung im quantenkinetischen Regime direkt gemessen werden. Die quasi-instantane Beschleunigung von Leitungsbandelektronen im polaren Galliumarsenid-Gitter und die anschließende Messung der Transmission im mittleren InfrarotBereich, zeigen eine Modulation der Transmission entlang der Anrege-Abtast Verzögerung mit der Frequenz des LO Phonons. Diese Oszillation ist ein direktes Maß der relativen Phase zwischen der Elektronenbewegung und der umgebenden Phonon Wolke. Quantenkinetische Modellrechnungen reproduzieren vollständig die beobachteten Effekte.

\section{Schlagwörter:}

ultraschnelle Spectroskopie, THz Erzeugung, Galliumarsenid, Quanten-kinetischer Ladungsträgertransport,flache Störstellen 


\section{Contents}

1 Introduction 1

$2 \mathrm{THz}$ field response of n-type gallium arsenide 5

2.1 General properties of gallium arsenide . . . . . . . . . 5

2.1.1 Band structure . . . . . . . . . . . . 5

2.1.2 Phonon spectrum . . . . . . . . . . . . 6

2.1.3 Doping and shallow impurities . . . . . . . . . 6

2.1.4 Coherent interaction of $\mathrm{THz}$ pulses in gallium arsenide 12

2.2 Open questions and perspective . . . . . . . . . . . 17

2.3 Electron transport in n-type gallium arsenide . . . . . . . . . 18

2.3.1 Quantum mechanical electron transport . . . . . . 18

2.3.2 Boltzmann transport model . . . . . . . . . . . . . 19

2.3.3 Nonlinear electron transport in static external electric fields . . . . . . . . . . . . . . . 24

2.4 Electron-phonon interaction . . . . . . . . . . . . 28

2.4.1 Polar optical mode scattering . . . . . . . . . . . 28

2.4.2 The electron in a dressed state: Polaron . . . . . . . 30

2.4.3 Experimental studies of quantum kinetic phenomena . 31

\section{Ultrafast nonlinear spectroscopy in the $\mathrm{THz}$ and mid-infrared} range $\quad 37$

3.1 Optical pulse generation and shaping . . . . . . . . . . 38

3.1.1 Ti:sapphire laser system . . . . . . . . . . . . . . 38

3.2 Characterization of MIR and THz pulses: Electro-optic sampling 40

3.2.1 Response function of the electro-optic sampling detector 43

3.3 Pulse generation at $\mathrm{THz}$ and mid-infrared frequencies . . . . . 51

3.3.1 Nonlinear optics and frequency mixing . . . . . . . 51

3.3.2 $\mathrm{THz}$ pulses generated in laser-induced plasma . . . . . 56

$3.4 \mathrm{THz}$ transmission spectroscopy on thin films . . . . . . . . . 64

3.5 Time-resolved two color pump-probe spectroscopy . . . . . . . 66

3.5.1 THz-pump-MIR-probe setup . . . . . . . . . . . 67 
4 High field $\mathrm{THz}$ response of n-type gallium arsenide at room $\begin{array}{ll}\text { temperature } & 69\end{array}$

4.1 Sample geometry . . . . . . . . . . . . . . . . . . . . . . 69

4.2 Experimental Results . . . . . . . . . . . . . . . 70

4.3 Discussion . . . . . . . . . . . . . . . . . 76

4.3.1 Qualitative discussion . . . . . . . . . 76

4.3.2 Quantum mechanical discrete state model (QMDS) . . 79

5 Rabi flopping on radiatively coupled shallow donor transitions $\quad 85$

$5.1 \mathrm{THz}$ transmission spectroscopy in vacuum . . . . . . . . . 85

5.2 Experimental results . . . . . . . . . . . . . . . . . 86

5.3 Shallow donors as two level systems . . . . . . . . . . . . 91

6 Internal motions of a quasiparticle governing its ultrafast nonlinear response $\quad 99$

6.1 n-type GaAs sample and experimental setup . . . . . . . . . . 99

6.2 Discussion . . . . . . . . . . . . . . . . . . . . 104

6.2.1 Qualitative discussion ............. . . 104

6.2.2 Quantum kinetic description of the internal polaron dynamics . . . . . . . . . . . . . . 109

6.2.3 Model calculations . . . . . . . . . . . . . 114

$\begin{array}{lll}7 & \text { Summary } & 119\end{array}$

A List of abbreviations 123

B Charge transport and optical absorption 125

C Electro-optic sampling without synchronization $\quad 127$

D Polarization dynamics in an ensemble of radiatively coupled $\begin{array}{ll}\text { two-level systems } & 131\end{array}$

E Derivation of the Heisenberg equations of motion 133

F Taylor expansion of the expectation value $\left\langle P_{\vec{q}} \sin (\vec{q} \cdot \vec{r})\right\rangle \quad 139$ 


\section{Chapter 1}

\section{Introduction}

The $\mathrm{THz}$ spectral range is of particular importance for semiconductor physics since it covers a wide range of elementary excitations such as phonons, bulk plasmons, internal and bound-continuum transitions of hydrogenic impurities and excitons as well as inter-Landau level transitions in magnetic fields. These transitions are characterized by the high dipole moment and the low effective carrier mass. In addition, $\mathrm{THz}$ spectroscopy provides insight into the free carrier response to an external electric field. The electric field strength applicable to a sample can be extremely high if the applied $\mathrm{THz}$ pulses are short enough to prevent irreversible damage due to the induced high current densities. For instance, an electric field of $10 \mathrm{kV} / \mathrm{cm}$ induces a current density of more than $50 \mathrm{MA} / \mathrm{cm}^{2}$ at doping densities around $10^{17} \mathrm{~cm}^{-3}[1]$. High-field $\mathrm{THz}$ spectroscopy is therefore a promising approach to study the excitation and relaxation dynamics on low energetic transitions and free carrier transport in semiconductors.

Besides the theoretical interest, high electric field effects in semiconductor devices are of growing importance in modern electronics. Since the invention of the first transistor 60 years ago in 1948 [2], semiconductor physics has seen a revolutionary expansion of research and technological development. The constant progress is best expressed in Moore's famous prediction that the number of transistors on a microchip doubles every 18 months [3]. Moore's law proved correct over the past 30 years, and the transistor gate length in conventional microchips shrunk to less than $50 \mathrm{~nm}$ [4]. Together with the miniaturization comes an increase of the internal electric field strength in modern devices and an increase in the operation frequency of up to $300 \mathrm{GHz}$ [5]. Hence, high field effects at frequencies approaching $1 \mathrm{THz}$ are a growing issue for the development in modern electronics.

Other devices operating at high internal electric THz fields are semiconductor sources for $\mathrm{THz}$ radiation, so-called quantum cascade lasers (QCL) 
$[6,7]$. These devices depend on a thorough understanding of the internal electron propagation under high field conditions.

In this thesis the physics of free and weekly bound electrons in a semiconductor lattice is investigated in strong electric fields. The best method to study the field response of the electronic system is time domain $\mathrm{THz}$ spectroscopy (TDTS) [8]. Its main advantage is the ability to fully resolve the electric field in amplitude and phase. This allows for a complete, contactless characterization of the material. In order to extend the research to the nonlinear regime, a novel technique for the generation of $\mathrm{THz}$ transients with high field amplitudes has been applied. The developed $\mathrm{THz}$ source yields ultrashort, sub single-cycle $\mathrm{THz}$ pulses. The following topics are studied in this thesis:

\section{(i) Weakly bound electrons in strong electric fields}

Control of the conductivity via the insertion of donor atoms is fundamental for the operation of semiconductor devices. These substitutional atoms modify the potential landscape in their vicinity, giving rise to bound electronic states of the donor atoms. In this thesis, the interplay of localized and free conduction band carriers is studied at different temperatures and different doping concentrations.

\section{(ii) High field electron transport in gallium arsenide at room tem- perature}

At room temperature high field electron transport in a polar lattice such as gallium arsenide is dominated by the Coulomb interaction between the electron and polar optical phonon modes of the crystal lattice. The electron mobility is drastically modified when the particle reaches a kinetic energy which corresponds to the energy of the lattice vibration. However, an experimental study in that regime is difficult due to the reststrahlen band at the phonon resonance. In this thesis, two color THz pump MIR probe spectroscopy is applied to investigate the electron polar optical phonon interaction on ultrashort time scales. The interaction is resonant at electron energies comparable to the energy of the LO phonon.

\section{Outline of this thesis:}

The material system investigated is n-type gallium arsenide. The basic properties of this material and the theoretical background on electrons bound to silicon impurities and electron transport in an electric field is discussed in Chapter 2. The experimental methods, which make extensive use of ultrashort laser pulses provided by a Ti:sapphire oscillator-chirped pulse am- 
plifier laser system, are discussed in the Chapter 3 from a technical point of view. The focus lies on the generation and detection of ultrashort pulses in the mid-infrared and $\mathrm{THz}$ range. In addition, the thin-film geometry which was used to extract the response of the measured sample is introduced and the experimental setup is presented together with the concept of two-color pump-probe spectroscopy. Chapters 4 and 5 present experimental studies of electrons bound to impurity atoms in an intense THz field. Strong THz transients with field strength above the impurity ionization threshold are applied to a n-doped gallium arsenide sample in Chapter 4 . The coherent sample response after the excitation pulse is detected and compared to model calculations of a single particle model. In the experiments described in Chapter 5, weak $\mathrm{THz}$ pulses are applied, where the amplitude is subsequently increased to field strength close to and slightly above the theoretical ionization field strength. In this experiment, the coherent interaction of the sample with the pump pulse is observed and modeled. In Chapter 6, a high field electron transport study is presented which uses THz pump MIR probe spectroscopy. This technique allows to study the electron mobility at kinetic energies comparable to the energy of the optical phonon in gallium arsenide. The experimental data are compared to quantum kinetic model calculations. 


\section{Chapter 2}

\section{$\mathrm{THz}$ field response of n-type gallium arsenide}

This chapter gives a summary of the basic properties of gallium arsenide with a special focus on the $\mathrm{THz}$ response. After a general overview, n-doping in gallium arsenide is discussed in some detail in the first part of this chapter.

The second part deals with the transport properties of free conduction band electrons in externally applied electric fields.

\subsection{General properties of gallium arsenide}

\subsubsection{Band structure}

Gallium arsenide is a III-V semiconductor compound with a zinc-blende crystal structure and a lattice constant $\mathrm{a}_{l}=0.56 \mathrm{~nm}$. The gallium arsenide unit cell is depicted in Figure 2.1 (a) and the band structure is shown in Figure 2.1 (b). The direct, dipole allowed band gap lies in the $\Gamma$-valley in the zone center. The separation between the conduction band minimum and the valence band maximum is $1.42 \mathrm{eV}$ [10] at room temperature. At the $\Gamma$-point, the valence band is four fold degenerate and splits into a light-hole and a heavy-hole band for higher wavevectors. The degeneracy in the zone center is lifted by stress or quantum confinement, e.g., in quantum wells [11]. A split-off band lies $0.34 \mathrm{eV}$ below the valence band maximum.

The conduction band is spherically symmetric at the zone center. The parabolic approximation in the $\Gamma$-valley yields a band dispersion which is described by a scalar effective mass $\mathrm{m}_{\text {eff }}=0.067 \mathrm{~m}_{e}$, where $\mathrm{m}_{e}$ is the free electron mass. Other local conduction band minima (L-, X-valley) are found at high symmetry points at the zone boundary. 

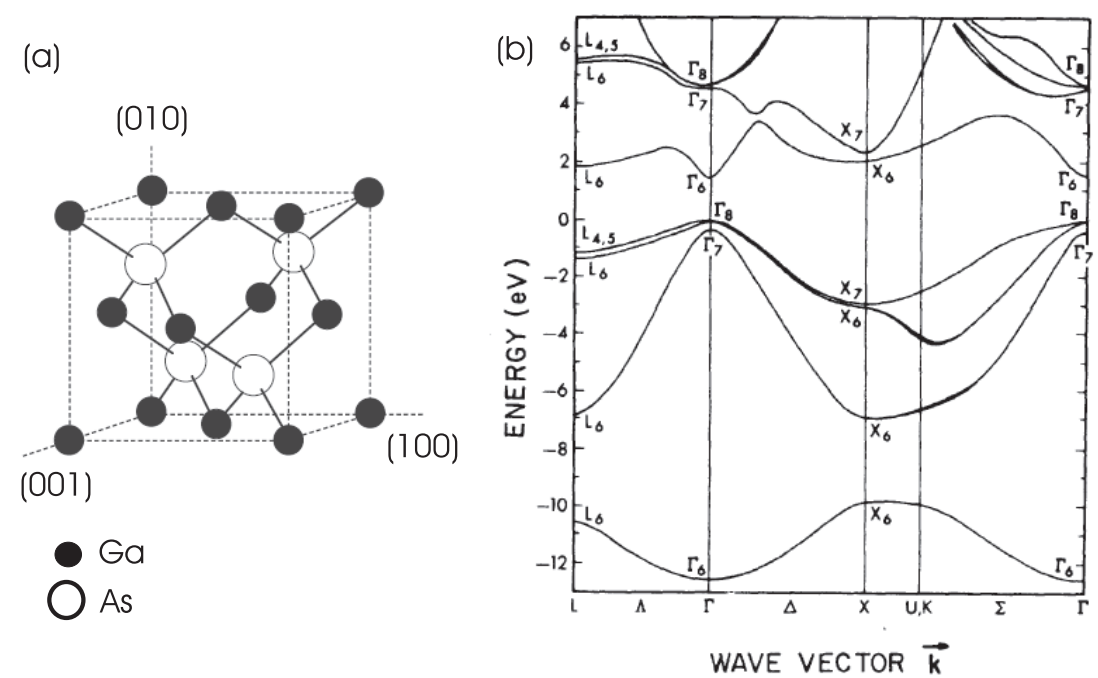

Figure 2.1: Crystal structure of gallium arsenide: (a) Unit cell (b) electronic band structure [9].

\subsubsection{Phonon spectrum}

The phonon spectrum of gallium arsenide is shown in Figure 2.2 [12]. It consists of three optical and three acoustic modes. The energy at the $\Gamma$-point is $33.25 \mathrm{meV}$ for the transversal optical mode and $36.14 \mathrm{meV}$ for the longitudinal optical mode at room temperature. At liquid helium temperature, the energies are $33.88 \mathrm{meV}$ and $36.86 \mathrm{meV}$, respectively.

\subsubsection{Doping and shallow impurities}

Doping is an essential technique for semiconductor electronic devices because it controls the material's conductivity. The insertion of donor atoms in the lattice of a semiconductor leads to localized hydrogenic states below the conduction band edge, which play an important role in the system response to a $\mathrm{THz}$ field. The doping atoms are also referred to as impurities.

\section{Hydrogenic donors in gallium arsenide}

A section of a gallium arsenide crystal doped with silicon is shown in Figure 2.3. In an undoped gallium arsenide lattice three valence electrons from the gallium atoms and five valence electrons from the arsenic atoms form the valence band of the crystal, which is entirely filled. By n-doping the material, a silicon atom having four valence electrons, substitutes a gallium 


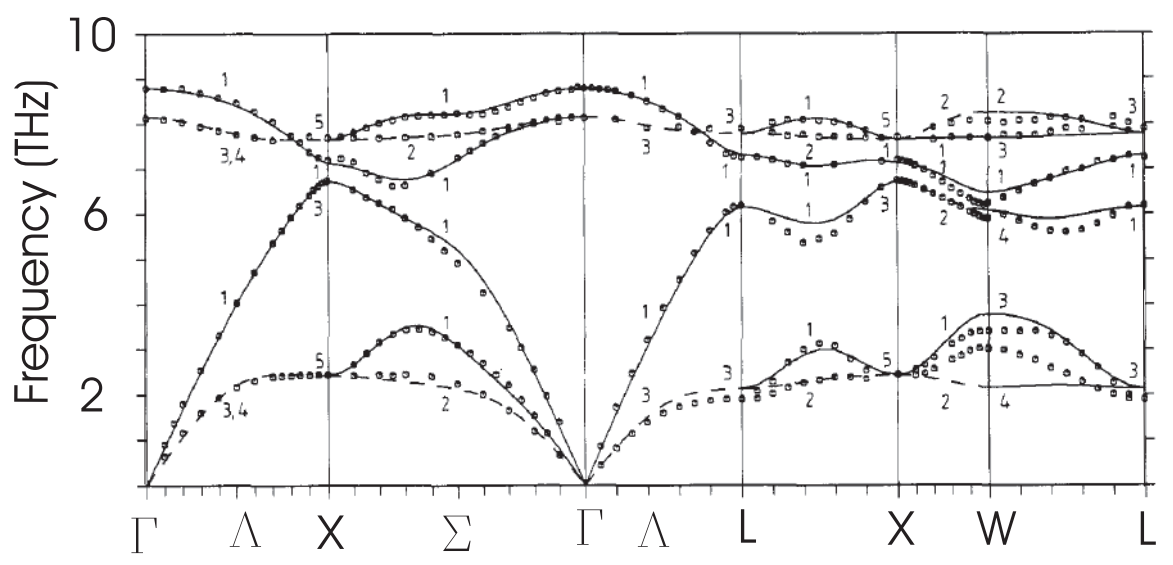

Figure 2.2: Phonon dispersion curves in gallium arsenide along high-symmetry axes [12].

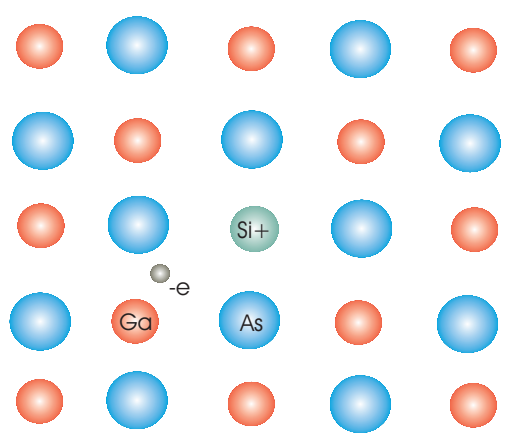

Figure 2.3: Si donor ion (green) replacing a neutral Ga atom (red) thus providing a free electron to the lattice. The As atoms are depicted in blue. 

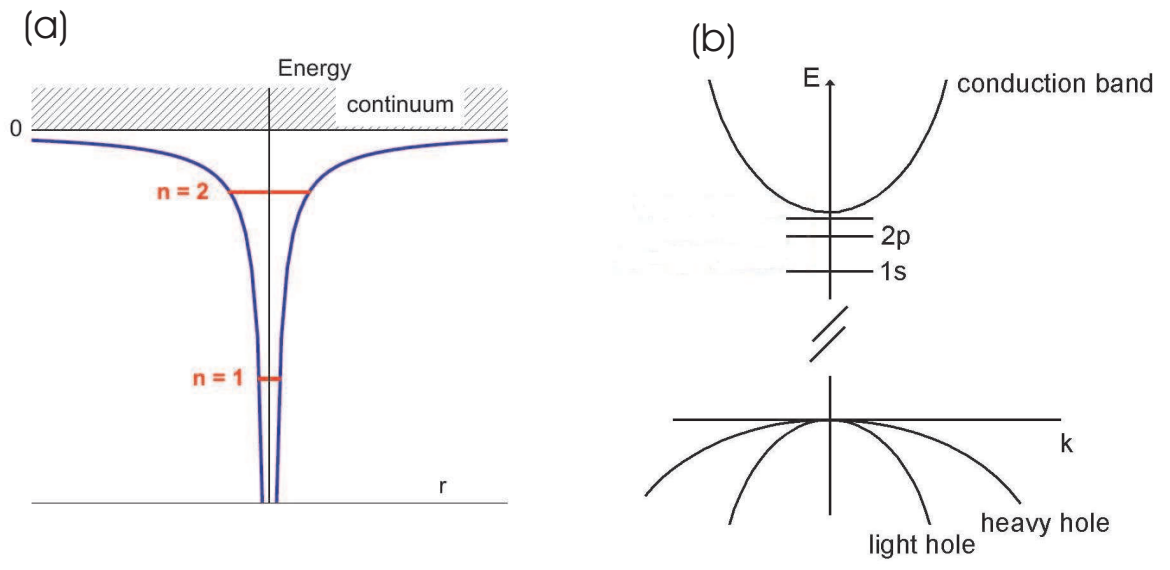

Figure 2.4: (a) Attractive potential of the silicon donor. Bound states exist for low energetic carriers. The continuum states lie in the conduction band. (b) Band structure of a doped gallium arsenide crystal within the effective mass approximation. Bound localized donor states $(n=1, n=2$ in (a)) lead to discrete states below the conduction band edge.

atom. Hence, the additional valence electron of the silicon atom populates conduction band states of the crystal.

An exact calculation of the screened silicon potential is very complicated as it contains many-body interactions between the valence electrons of the host and the silicon electrons. However, a reasonable approximation for the potential is to assume the positive charge of the donor to be screened by the dielectric constant of the host crystal [13]. The approximation is valid in the case that the Coulomb potential $V(r)$ is spread over a large area, i.e., $r \gg a_{l}$. The Coulomb potential of the donor then reads:

$$
V(r)=\frac{e^{2}}{4 \pi \epsilon_{0} \epsilon_{\mathrm{GaAs}}|r|},
$$

with $\epsilon_{0}$ and $\epsilon_{\mathrm{GaAs}}$ being the free space and gallium arsenide dielectric constant and $e$ is the elementary charge of the electron, respectively. Insertion of the potential (2.1) in the Hamiltonian describing an electron propagating in a crystal lattice in the Born-Oppenheim approximation yields the Schrödinger equation for the hydrogenic impurity:

$$
\left[-\frac{\vec{p}^{2}}{2 m_{\mathrm{eff}}}+\frac{e^{2}}{4 \pi \epsilon_{0} \epsilon_{\mathrm{GaAs}}|r|}\right] \phi(r)=E \phi(r) .
$$

$\phi(r)$ denote the envelope function of the conduction band electron and $\vec{p}$ is the electron momentum, respectively. 
Equation (2.2) resembles the Hamiltonian of an isolated hydrogen atom, except for the parameters $m_{\text {eff }}$ and $\epsilon_{\mathrm{GaAs}}$. It can be solved analytically and yields a Rydberg series for the energies of the bound states with a modified Rydberg constant $\mathrm{R}_{y}^{\prime}$

$$
\begin{aligned}
& E_{n}=-\frac{R_{y}^{\prime}}{n^{2}} \quad n=1,2,3, \ldots \\
& R_{y}^{\prime}=\frac{e^{4} m_{\mathrm{eff}}}{32 \hbar^{2} \pi^{2} \epsilon_{0}^{2} \epsilon_{\text {GaAs }}^{2}},
\end{aligned}
$$

where $\hbar=h / 2 \pi$ denotes the normalized Planck's constant. The extent of the bound state electron wavepacket is expressed through a modified Bohr radius

$$
a_{B}=\frac{4 \pi \epsilon_{0} \epsilon_{\mathrm{host}} \hbar^{2}}{m_{\mathrm{eff}} e^{2}}
$$

Figure 2.4 (a) depicts the donor potential in real space and Figure 2.4 (b) sketches the band structure of a doped semiconductor. The bound electronic states below the conduction band edge are marked in analogy to the hydrogen atom $1 \mathrm{~S}, 2 \mathrm{P}, \ldots$ The large extension of these states in momentum space correspond to confined, localized states in real space. For Si-doped gallium arsenide with $\epsilon_{\mathrm{GaAs}}=12.6$ one finds a donor binding energy of $R_{y}^{\prime} \approx 6 \mathrm{meV}$ and an effective Bohr radius of $\mathrm{a}_{B} \approx 10 \mathrm{~nm}$.

\section{Semiconductor statistics}

The relative density of ionized donors depends on the doping concentration and on the lattice temperature, i.e., the thermal energy [14]. The density of ionized donors, which corresponds to the density of free carriers in the conduction band, is given by

$$
N=\frac{N_{c}^{\prime}}{2}\left(\sqrt{1+\frac{4 N_{d}}{N_{c}^{\prime}}}-1\right),
$$

where $\mathrm{N}_{c}^{\prime}$ is

$$
N_{c}^{\prime}=\frac{1}{2}\left(\frac{m_{\mathrm{eff}} k_{B} T}{2 \pi \hbar^{2}}\right)^{3 / 2} \exp \left(\frac{\Delta E_{D}}{k_{B} T}\right)
$$

with the donor binding energy $\Delta E_{D}$ and $\mathrm{k}_{B}$ denoting the Boltzmann factor. A derivation of equation (2.5) is found in [15]. Figure 2.5 shows the relative 


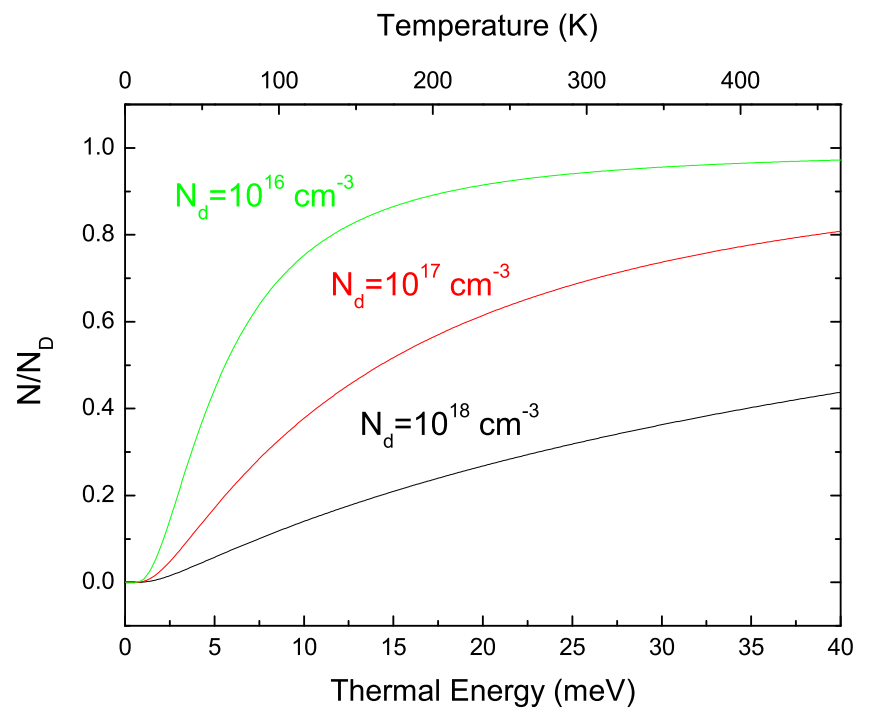

Figure 2.5: Relative density of free carriers over temperature for $N_{d}=10^{16} \mathrm{~cm}^{-3}$ (green), $N_{d}=10^{17} \mathrm{~cm}^{-3}$ (red) and $N_{d}=10^{18} \mathrm{~cm}^{-3}$ (black) in an n-doped gallium arsenide lattice.

density of ionized impurities in silicon doped gallium arsenide over the temperature for three different doping concentrations $N_{d}=10^{16} \mathrm{~cm}^{-3}, 10^{17} \mathrm{~cm}^{-3}$ and $10^{18} \mathrm{~cm}^{-3}$ (green, red and blue, respectively) as calculated from equation (2.5). Because only unbound carriers can participate in charge transport, even doped semiconductors can become isolating at very low temperatures $\left(\mathrm{N} / \mathrm{N}_{d} \approx 0\right)$. On the other hand, even at room temperature, which corresponds to a thermal energy of approximately $26 \mathrm{meV}$ not all donor atoms are ionized $\left(\mathrm{N} / \mathrm{N}_{d}<1\right)$. The higher the doping concentration, the higher is the remaining population in the impurity ground states at a given temperature.

\section{Impurity conduction and impurity bands}

The electronic wavefunction in the donor ground state is significantly larger than the lattice constant. At intermediate and high doping concentrations, where the donor Bohr radius $\mathrm{a}_{B}$ is on the same order as the inter-impurity distance $\Delta_{\text {imp }}$, the probability for an impurity ground state electron to stretch over neighboring impurities can become important. It can be shown that a screened Thomas-Fermi potential has bound states for screening length $\mathrm{k}_{s}<1.19 / \mathrm{a}_{B}[16,17]$. A simple cubic lattice would be isolating, if $\Delta_{\text {imp }}>2.28 \mathrm{a}_{B}$. Experimental data of the metal-insulator transition (MIT) in semiconductors 


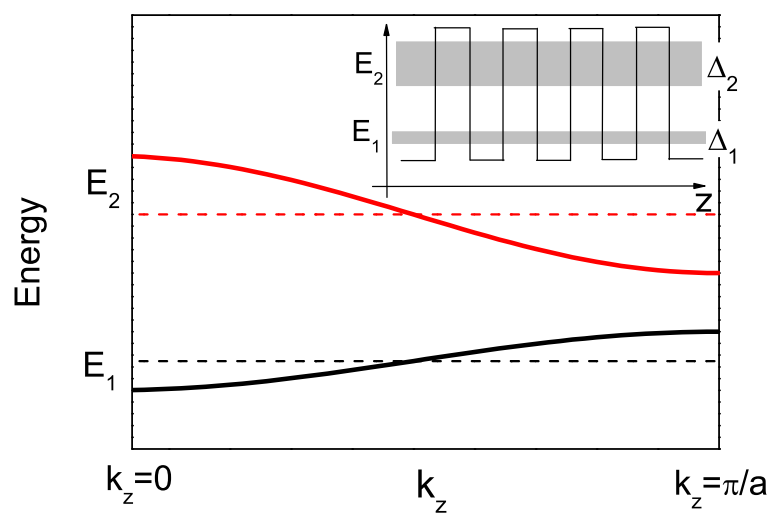

Figure 2.6: Miniband dispersion (solid lines) in a semiconductor superlattice calculated with the Kroenig-Penny model. The dashed lines show the energy of the ground state and the first excited state of an isolated quantum well of the same width. The potential of the superlattice is depicted in the inset.

are rare. A sharp MIT was observed in p-doped silicon. The conductivity increased by more than $10^{3}$ for a variation of the carrier concentration $n_{c}$ of less than $1 \%$ [18]. The critical density observed was $3.74 \times 10^{18} \mathrm{~cm}^{-3}$. A similar value was found in [19].

An interesting aspect of carrier delocalization and the formation of impurity bands is whether or not this would influence the transition energies between the impurity states. A simple one-dimensional model for a quick investigation is the Kroenig-Penney model found in many quantum mechanics textbooks [20]. It describes the miniband formation in coupled rectangular potentials similar to a semiconductor superlattice. The potential is depicted in the inset of Figure 2.6. The subband dispersion can be calculated with the following expression [21]:

$$
E_{n}(\vec{k})=E_{n}+\frac{\Delta_{n}}{2}\left(1+(-1)^{n} \cos \left(k_{z} d\right)\right)+\frac{\hbar^{2}\left[k_{x}^{2}+k_{y}^{2}\right]}{2 m_{\mathrm{eff}}} .
$$

Here, $\Delta_{n}, d, k_{z}$ and $k_{x, y}$ denote the energetic width of the miniband, the barrier thickness and the wavevector in the confined and unconfined direction, respectively.

Figure 2.6 shows the calculated miniband dispersion for the two lowest minibands (solid lines) in the $\mathrm{k}_{z}$-direction compared to the energy levels of an isolated quantum well (dashed lines) of the same size. The transition energy 
increases for low $\vec{k}$-vectors, i.e., in the zone center and decreases below the value of the isolated potential at the zone boundary. If the miniband is completely filled, one observes a broad absorption spectrum because optical transitions occur over the whole superlattice Brillouin zone. However, if the miniband is only half filled, the optical absorption spectrum is blueshifted compared to an isolated quantum well [22].

The impurity absorption spectra in doped semiconductors hence depend on the doping concentration. Since the impurities are randomly distributed in the crystal lattice, a delocalization of the ground state wavefunction over more than one impurity can occur even at rather low doping concentrations. This would be the case if the local inter-impurity distance is below the average. At the same time the disordered potential also counteracts a complete delocalization into impurity bands since a periodicity in the impurity distribution is not given over a larger area. Hence, the electron wavefunction is localized only over neighboring impurities even at higher doping concentrations. This effect is called Anderson localization [23].

\subsubsection{Coherent interaction of $\mathrm{THz}$ pulses in gallium arsenide}

Impurity systems are characterized by electronic transitions in the impurity potentials. In the following section, the coherent interaction of $\mathrm{THz}$ light with electrons bound to impurity atoms is described. The coherent regime during and immediately after the exciting pulse is characterized by a macroscopic polarization with a definite phase relative to the excitation. This macroscopic polarization determines the linear and nonlinear response of the system. Several coherent spectroscopy techniques have been developed in the last decades [24]. The coherent regime is interesting because it exhibits some of the most fundamental quantum mechanical processes like interference and superposition phenomena. In addition it allows the study of various scattering processes, which are responsible for the loss of coherence and the extraction of scattering rates in semiconductors.

\section{Density matrix of an ensemble of independent two-level systems}

The impurity system can be modeled approximately as an ensemble of independent two-level systems. The lower and upper states correspond to the $1 \mathrm{~S}$ and 2P states of the impurity system, respectively. Neglecting other states is reasonable because the $1 \mathrm{~S} \rightarrow 2 \mathrm{P}$ transition has the highest electric dipole moment in the impurity transition spectrum. The interaction of the ensemble of two level systems with an incident $\mathrm{THz}$ pulse is described by the density 
matrix [20]. The diagonal elements of the density matrix $\varrho_{i i}(\mathrm{i}=1,2)$ denote the occupation probability of the two energy eigenstates and the off-diagonal elements $\varrho_{i j}(\mathrm{i}, \mathrm{j}=1,2 ; \mathrm{i} \neq \mathrm{j})$ represent the coherence of the population between the upper and lower state, respectively. The density matrix does not yield the quantum mechanical state of the ensemble but gives certain information in terms of occupation probabilities and coherence in the system. In an ensemble of two level systems, the elements of the density matrix fulfill the relations:

$$
\begin{aligned}
\varrho_{12} & =\varrho_{21}^{*}, \\
\varrho_{11}+\varrho_{22} & =1 \\
\left|\varrho_{12}\right|=\left|\varrho_{21}\right| & \leq \sqrt{\varrho_{11} \varrho_{22}} .
\end{aligned}
$$

The time evolution of the density matrix follows the Liouville equation [25]:

$$
\frac{\partial \varrho}{\partial t}=\frac{i}{\hbar}[\varrho, H] \text {. }
$$

The Hamiltonian is composed of a term $\mathrm{H}_{0}$ which describes the system in absence of an electric field, and of the interaction term $\mathrm{H}^{\prime}=-\vec{d} \cdot \vec{E}(t)$, where $\vec{d}$ is the electric dipole matrix which has the form: $d_{11}=d_{22}=0$ and $d_{12}=d_{21}$. The time evolution of the non-diagonal elements of the density matrix, which describe the polarization, becomes [26]:

$$
\frac{\partial \varrho_{12}}{\partial t}=\left(i \omega_{0}-\frac{1}{T_{2}}\right) \varrho_{12}-i \frac{\vec{d}}{\hbar} E(t)\left(1-2 \varrho_{22}\right) .
$$

The time evolution of the excited state population $\varrho_{22}$ follows

$$
\frac{\partial \varrho_{22}}{\partial t}=-\frac{\varrho_{22}}{T_{1}}-\frac{\vec{d}}{\hbar} E(t) \operatorname{Im}\left(\varrho_{12}\right)
$$

where the time constants $T_{1}$ and $T_{2}$ are phenomenological population relaxation and dephasing time constants, which result from the interaction of the ensemble with the surrounding reservoir. The system of coupled partial differential equations (2.10) and (2.11) is called the Optical Bloch Equations (OBEs).

\section{Bloch vector representation}

An intuitive graphical representation of the OBEs is the Bloch vector $\vec{r}$ :

$$
\vec{r}=\left(\begin{array}{c}
r_{1} \\
r_{2} \\
r_{3}
\end{array}\right)=\left(\begin{array}{c}
2 \operatorname{Re}\left(\varrho_{21}\right) \\
-2 \operatorname{Im}\left(\varrho_{21}\right) \\
\varrho_{22}-\varrho_{11}
\end{array}\right) .
$$




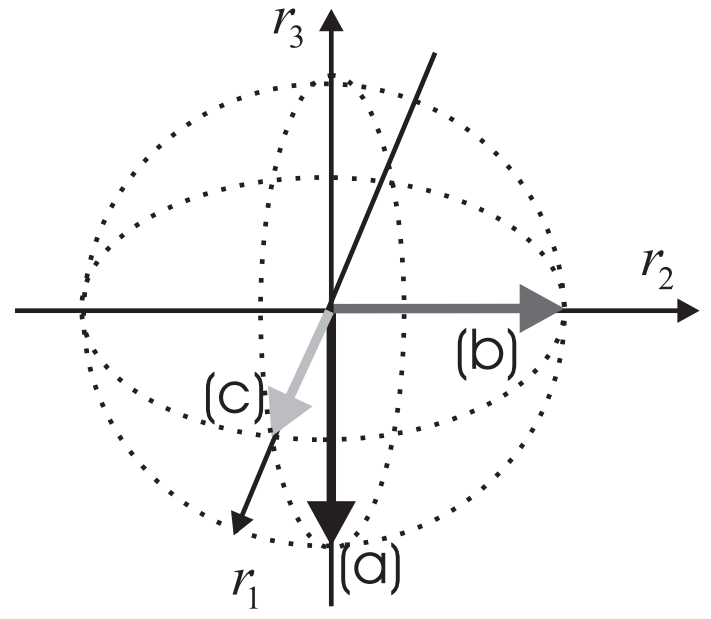

Figure 2.7: Bloch vector of an atomic ensemble in (a) ground state, (b) maximum polarization and coupling to the driving field and (c) maximum polarization but no coupling to the driving field.

It allows the representation of the population and polarization state of an ensemble of two level systems in a sphere. The vector component $r_{3}$ is of particular interest, as it depicts the population in the lower and upper level. An example is given in Figure 2.7 (a), which shows an ensemble in the ground state. Here $r_{3}=-1$ and all polarization components are zero. In the case that the coherence in the ensemble is maximal, the Bloch vector will always rotate on the surface of the sphere, i.e., $|\vec{r}|=1$. Less coherence manifests in a Bloch vector which satisfies $|\vec{r}|<1$. An important variable is the rotation frequency $\Omega$ around the $r_{1}$ axis. It is called the Rabi frequency and describes an oscillation of the population between the lower and the upper state. The Rabi frequency is defined as:

$$
\Omega=-\frac{\vec{d} \vec{E}}{\hbar}
$$

The polarization in the ensemble is maximal if the Bloch vector rotates on the surface of the sphere in the $r_{1}-r_{2}$-plane. This situation is depicted in Figure 2.7, vectors (b) and (c). The expectation value of the microscopic polarization is also obtained from the density matrix

$$
\langle\vec{d}\rangle=\operatorname{Tr}(\varrho \vec{d})=\vec{d}\left(\varrho_{12}+\varrho_{21}\right)
$$

and the macroscopic polarization is obtained by summation over the $n$ individual two-level systems. That is:

$$
P=n\langle\vec{d}\rangle .
$$



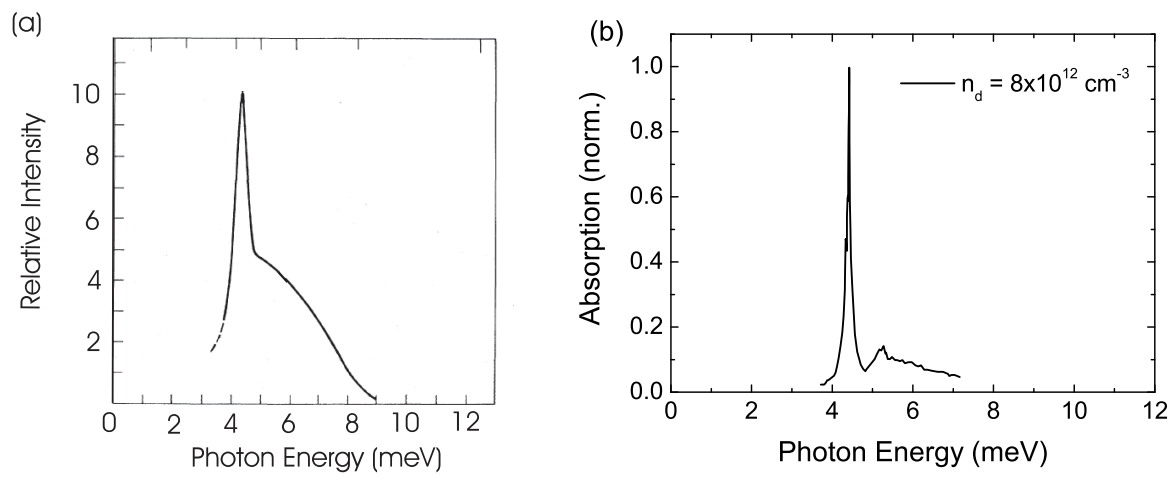

Figure 2.8: (a) Photoluminescence spectrum of impact-ionized n-type gallium arsenide $\left(N_{d}=2.5 \times 10^{14} \mathrm{~cm}^{-3}\right)$ at a lattice temperature of $4.2 \mathrm{~K}$ taken from reference [37]. The $\mathrm{PL}$ peak corresponds to the $2 \mathrm{P} \rightarrow 1 \mathrm{~S}$ transition, the high-energetic components result from recombination between continuum states and ground states. (b) Photocurrent measurement of a GaAs sample with a doping concentration of $N_{d}=8 \times 10^{12} \mathrm{~cm}^{-3}$ at $\mathrm{T}=4.2 \mathrm{~K}$ taken from reference [32].

\section{THz spectroscopy of shallow donors}

The physics of impurity transitions in semiconductors gained broader interest in the 1940's when scientists searched for possible detectors for long wavelength radiation $[13,27]$. Theoretical works predicted a hydrogen-like absorption spectrum from shallow donors and acceptors [28, 29, 30], which makes them an interesting model for atomic systems [31]. Experimentally, isolated impurities were studied via photocurrent (PC) $[32,33,34]$ and photoluminescence (PL) $[35,36,37]$ measurements. PC measurements exploit the fact that carriers in the impurity ground state do not contribute to charge transport. The measured conductivity is therefore proportional to the population in excited impurity states. In the PL measurements the incoherent recombination radiation from the impurities is spectrally detected [38]. Typically these experiments were done at liquid helium temperatures. A farinfrared PL spectrum of impact-ionized donors in Cr-doped gallium arsenide measured by Melngailis et al. [37] is shown in Figure 2.8 (a). It shows a distinct PL peak, which corresponds to the donor exited state to donor ground state transition. In addition, a recombination band from higher impurity and continuum states to the impurity ground state was observed. Figure 2.8 (b) shows a PC measurement of n-doped gallium arsenide with an even lower doping concentration of $N_{d}=8 \times 10^{12} \mathrm{~cm}^{-3}$. Again, a distinct peak corresponding to an impurity ground state to excited state transition and a 
broader band corresponding to continuum transitions was observed.

\section{Nonlinear spectroscopy on shallow donor transitions}

In nonlinear spectroscopy experiments, the splitting of the impurity states in a magnetic field was used to tune the transition energy to a coherent radiation source $([39,40]$ and references therein). By changing the resonance conditions, a significant modification of the excited state lifetime was observed [41]. The lifetime changes from around $10 \mathrm{~ns}$ in resonance to approximately $500 \mathrm{~ns}$ under off-resonance conditions, a precondition for lasing in a shallow impurity system. The saturation intensities of the $1 \mathrm{~s} \rightarrow 2 \mathrm{p}$-transition were shown to increase with decreasing magnetic field, i.e., for lower resonance frequencies [42].

The magneto-impurity effect was also utilized to measure the free induction decay of the donor ground-to-excited state transition in PC experiments [43]. For resonant excitation, a coherent excited state population was traceable up to 50 ps after excitation, compared to 5 ps for off-resonant excitation. Coherent manipulation of the excited state population and Rabi oscillations on donor transitions have been demonstrated [44, 45, 46], which suggest shallow impurities as model systems for quantum information processing. Recently, an optical method for the direct readout of the donor ground state population was proposed [47], which exploits the high scattering contrast between electrons in the donor ground and excited states with excitons.

Far-infrared lasing was observed on transitions between Landau levels of the light-hole and heavy-hole valence band in p-type germanium in crossed electric and magnetic fields [48, 49]. This mechanism provides narrow band far-infrared generation which is linearly tunable by the magnetic field strength and yields output powers of several $100 \mathrm{~mW}[50,51]$. In the last decade p-type inter-valence band lasers were optimized to yield picosecond pulses $[52,53]$ at low magnetic fields [54]. A review on p-type germanium lasers is given in reference [55].

Electronic transitions in impurity potentials can be used to generate farinfrared radiation via stimulated emission from excited impurity states to the impurity ground state. The mechanism is depicted in Figure 2.9. In ndoped material, lasing was demonstrated on transitions in donor potentials in silicon, where a $\mathrm{CO}_{2}$-Laser was used as a pump source [56, 57]. Recently, stimulated emission in a phonon-assisted process was observed [58], which allows the use of $\mathrm{THz}$ radiation as a pump source. 


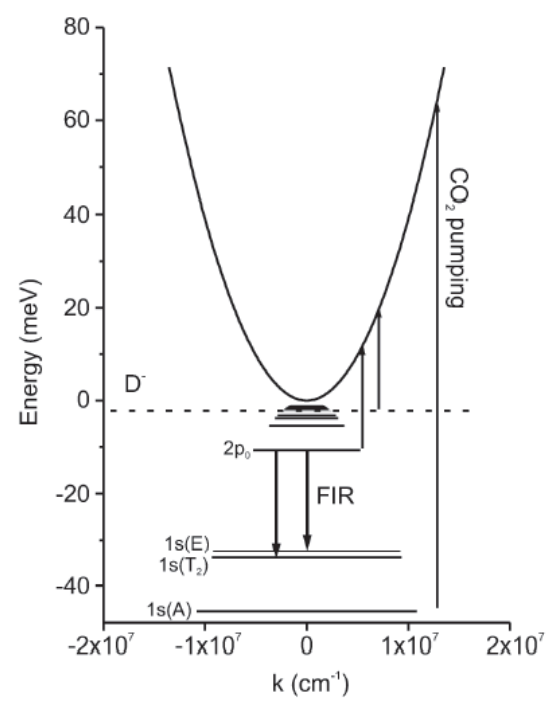

Figure 2.9: Far-infrared laser exploiting transitions in the impurity potential of a donor in n-doped silicon. $\mathrm{A} \mathrm{CO}_{2}$-laser is used as a pump source. Excited carriers recombine to the impurity ground state via the emission of coherent $\mathrm{THz}$ radiation. Sketch taken from reference [56].

\subsection{Open questions and perspective}

In the previous sections, the properties of donor atoms in gallium arsenide were discussed. These so-called impurity systems are of great interest: doping is essential for the properties of modern electronic devices, as it provides free carriers for charge transport. It is also known that the donor atoms modify the potential surface in their vicinity. These modifications lead to bound, localized states which can be used for radiative transitions and coherent population control, leading to new applications like $\mathrm{THz}$ lasers or storages for quantum information.

The low binding energy of the donor ground state of approximately $6 \mathrm{meV}$ in gallium arsenide and the high dielectric function combined with a low effective electron mass suggest that $\mathrm{THz}$ pulses can interact with shallow impurity systems via different mechanisms. The low binding energy leads to resonant interaction with $\mathrm{THz}$ radiation, thus inducing radiative transitions, which prefers the photon character of the $\mathrm{THz}$ light. In contrast, the high dielectric constant and the low effective electron mass lead to a low ionization field strength, which emphasizes the field character of the $\mathrm{THz}$ pulse in the interaction with the impurity system. While the description of the shallow impurity system is self-consistent in either of these two regimes, which are selected by the experimental conditions, e.g., temperature and doping 
concentration, the transition between the regimes is unexplored.

Here, ultrafast, highly intense $\mathrm{THz}$ pulses could provide new insights to the interplay between field driven and optical excitation. Following these ideas, such experiments are carried out in our lab for the first time. In Chapter 4 the response of n-type doped bulk gallium arsenide to a quasi single-cycle $\mathrm{THz}$ pulse with peak amplitudes of approximately $80 \mathrm{kV} / \mathrm{cm}$ is studied, elucidating the interplay between initially bound and unbound electronic states. In Chapter 5 the coherent interaction of ultrashort $\mathrm{THz}$ transients with bound carriers is driven to a limit by constantly increasing the $\mathrm{THz}$ amplitude up to the ionization of an initially neutral impurity.

\subsection{Electron transport in n-type gallium ar- senide}

In this section, electron transport in n-doped gallium arsenide is discussed. In contrast to the first part of this chapter which dealt with electrons bound to donor atoms, here the focus lies on free conduction band electrons.

\subsubsection{Quantum mechanical electron transport}

A complete quantum mechanical formulation of electron transport in a semiconductor is a problem of enormous complexity since it includes many-body interactions. The Hamiltonian including free conduction band electrons and longitudinal optical phonons expressed in second quantization reads [59]:

$$
\begin{aligned}
H & =\sum_{k} \epsilon_{k} c_{k}^{\dagger} c_{k}+\sum_{q} \hbar \omega_{L O} b_{q}^{\dagger} b_{q}+\sum_{k} c_{k}^{\dagger} e \vec{E} \frac{\partial}{\partial k} c_{k}+\sum_{k, k^{\prime}, q} V_{q} c_{k+q}^{\dagger} c_{k^{\prime}-q}^{\dagger} c_{k^{\prime}} c_{k} \\
& +\sum_{k, q}\left[g_{q} c_{k}^{\dagger} b_{q} c_{k-q}+g_{q}^{*} c_{k-q}^{\dagger} b_{q}^{\dagger} c_{k}\right] .
\end{aligned}
$$

$\epsilon, c^{\dagger}, c$ denote the electron energy and the electron creation and annihilation operators, $b^{\dagger}$ and $b$ denote the phonon creation and annihilation operators, $V$ is the Coulomb scattering potential, $\omega_{L O}$ is the frequency of the LO phonon and $k$ and $q$ denote the electron and phonon wavenumber. The five terms of the Hamiltonian denote the energy of the electrons and phonons, the electronelectric field interaction in the electric dipole approximation, the electronelectron interaction and the electron-phonon interaction, respectively, where $g_{q}$ denotes the electron phonon coupling coefficient given by

$$
g_{q}=-i\left[\frac{2 \pi^{2} \hbar \omega_{L O}}{\Omega}\left(\frac{1}{\epsilon_{\infty}}-\frac{1}{\epsilon_{s}}\right)\right] \frac{1}{q} .
$$


Here, $\epsilon_{\infty}, \epsilon_{s}$ and $\Omega$ represent the high frequency and static dielectric constant and a normalization volume, respectively.

Starting with the Hamiltonian in equation (2.15) the Quantum Boltzmann Equation can be derived [60], which is still too complex to be solved without making significant approximations. A commonly used formalism to study transport phenomena is the semiclassical Boltzmann equation which neglects completely any quantum coherence and quantum kinetic effects in transport processes $[61,60,62]$. However, despite the approximations made, the Boltzmann transport model can accurately describe linear and nonlinear transport phenomena on long time scales. It is described in the next section.

\subsubsection{Boltzmann transport model}

In the semiclassical Boltzmann transport theory, the electrons are described by a distribution function $f(\vec{r}, \vec{k}, t)$, which is in general a non-equilibrium distribution function. The temporal change of $f(\vec{r}, \vec{k}, t)$ is governed by the Boltzmann Transport Equation (BTE) [63]:

$$
\frac{d f}{d t}+\frac{1}{\hbar} \nabla_{\vec{k}} \epsilon(\vec{k}) \nabla_{\vec{r}} f+\frac{e}{\hbar}\left(\vec{E}-\frac{1}{\hbar} \nabla_{\vec{k}} \epsilon(\vec{k}) \times \vec{B}\right) \nabla_{\vec{k}} f=\left(\frac{\partial f}{\partial t}\right)_{\mathrm{coll}} .
$$

Here, $\vec{E}$ and $\vec{B}$ denote an external electric and magnetic field, respectively. The collision term on the right hand side of equation (2.17) describes the change of the distribution function due to collisions of the electrons at, e.g., phonons, impurities, dislocations and surfaces. The BTE represents a selfconsistent rate equation system for the population in various quantum mechanical states, i.e., an equation of motion for the electron distribution function. For a spatially homogeneous system the $\vec{r}$-dependence is neglected and $f(\vec{r}, \vec{k}, t)$ becomes $f(\vec{k}, t)$. Typically only an external electric field is assumed and the magnetic field is set to zero.

The most interesting term is the collision term on the right hand side of equation (2.17). The following collision integral

$$
\left(\frac{\partial f}{\partial t}\right)_{\text {coll }}=\int W\left(\vec{k}^{\prime}, \vec{k}\right) f(\vec{k})\left[1-f\left(\vec{k}^{\prime}\right)\right]-W\left(\vec{k}, \vec{k}^{\prime}\right) f\left(\vec{k}^{\prime}\right)[1-f(\vec{k})]
$$

where $W\left(\vec{k}^{\prime}, \vec{k}\right)$ denotes the scattering probability of an electron from state $\vec{k}$ to $\vec{k}^{\prime}$, is commonly evaluated by Fermi's Golden Rule. It is important to note that equation (2.18) is a serious approximation of the many-particle interaction process $[59,60]$. It consists in assuming an infinitely short duration of the scattering process and neglecting any coherence between the scattering partners. The actual time scale of a scattering event is determined by, e.g., 
the lattice response time, i.e., the inverse phonon frequency $\omega_{L O}^{-1}$. Hence, the so-called Markov-approximation which leads to equation (2.18) is valid for electron transport in dc and slowly varying electric fields with moderate amplitudes, where the electron movement is slow compared to the time scale of the scattering event [64].

The external electrical force accelerates an electron to a velocity

$$
\vec{v}(\vec{k})=\frac{1}{\hbar} \nabla_{\vec{k}} \epsilon(\vec{k}) .
$$

The average velocity of the distribution containing $\mathrm{N}_{d}$ electrons is

$$
\langle\vec{v}(\vec{E})\rangle=\frac{\int f(\vec{k}, \vec{E}) \vec{v}(\vec{k}) d \vec{k}^{3}}{\int f(\vec{k}, \vec{E}) d \vec{k}^{3}},
$$

where the non-equilibrium distribution function depends on the external field strength. With the ensemble velocity one can define a linear electron mobility

$$
\langle\vec{v}(\vec{E})\rangle=\mu \vec{E}+\cdots
$$

and a conductivity

$$
\sigma=N_{d} e \mu
$$

for the material. The collision term acts like a friction force against the acceleration of the electrons in the external field. Hence, the electric field leads to a finite steady-state current density

$$
\vec{j}=e \int v(\vec{k}) f(\vec{k}) d \vec{k}
$$

\section{The relaxation time approximation}

The collision term in equation (2.18) is often simplified by introducing a relaxation time $\tau$ :

$$
\left(\frac{\partial f(\vec{k})}{\partial t}\right)_{\text {coll }}=\frac{f_{\text {equ }}(\vec{k})-f(\vec{k}, t)}{\tau(\vec{k})} .
$$

It describes the relaxation of a non-equilibrium distribution to thermal equilibrium through scattering. The equilibrium distribution is characterized by a temperature and, in the case of a free electron gas, follows the Fermidistribution:

$$
f_{\text {equ }}(\vec{k})=\frac{1}{e^{\frac{\epsilon(\vec{k})-\epsilon_{F}}{k_{B} T}}+1} .
$$



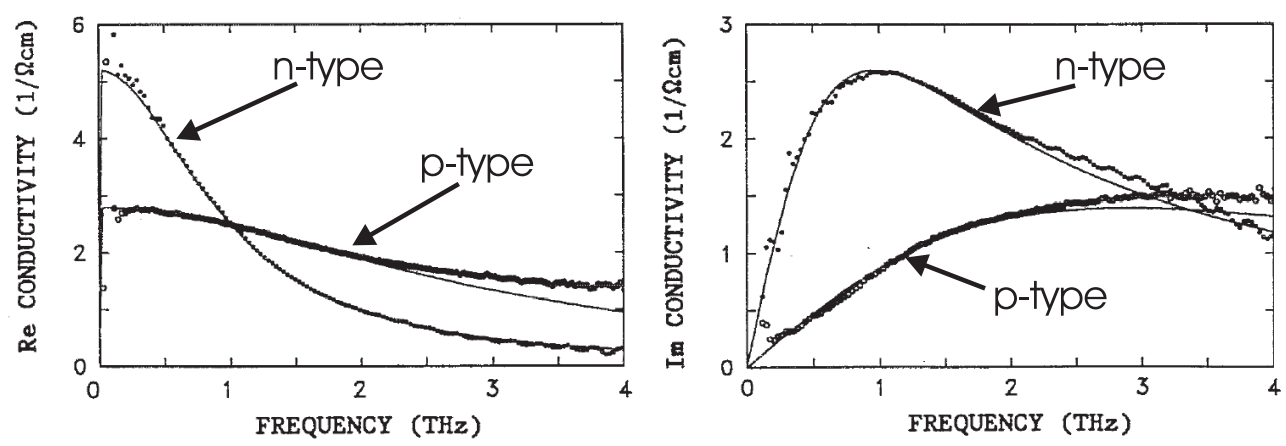

Figure 2.10: Frequency dependent conductivity of n-type and p-type GaAs compared to a Drude fit (solid line) showing good agreement for frequencies up to $4 \mathrm{THz}$. Data taken from reference [67].

Here $\epsilon_{F}$ and $T$ denote the the Fermi energy and the characteristic distribution temperature, respectively. In the relaxation time approximation, $\tau$ is independent of the structure of the non-equilibrium distribution function $f(\vec{k})$. The inverse relaxation time corresponds to a mean scattering rate $\Gamma=1 / \tau$.

\section{Electron transport in time-dependent electric fields: the Drude model}

The steady-state current density $\vec{j}$ fades out exponentially with the relaxation time $\tau$ when the dc external field $\mathrm{E}_{0}(\mathrm{t})$ is turned off at $t=0$

$$
\frac{\partial \vec{j}}{\partial t}=-\frac{\vec{j}}{\tau}+\frac{e}{m_{\mathrm{eff}}} \vec{E}(t) .
$$

In the frequency domain, this time dependance corresponds to a Lorentzian conductivity spectrum of the form

$$
\sigma(\omega)=\sigma_{d c} \frac{i \Gamma}{\omega+i \Gamma}
$$

Such a frequency response was predicted by Paul Drude using a ballistic electron transport model [65] and has been experimentally confirmed at low frequencies in doped semiconductors like n-doped silicon [66] or n- and pdoped gallium arsenide [67]. Results of linear $\mathrm{THz}$ transmission experiments on gallium arsenide are shown in Figure 2.10. The Drude fit (solid line) matches the measured conductivity of the gallium arsenide sample very well, showing that the Drude frequency response resulting from the relaxation time approximation is in good agreement with the linear $\mathrm{THz}$ response in n-type gallium arsenide. 


\section{Electron transport at mid-infrared frequencies}

At mid-infrared frequencies, the interaction of a medium with an external electric field is usually treated in an optical picture instead of a transport picture. For semiconductors with intermediate doping concentration of $\mathrm{N}_{d}<10^{17} \mathrm{~cm}^{-3}$ as studied here, the frequency dependent absorption coefficient $\alpha$ in the mid-infrared range is related to the frequency dependent mobility by

$$
\alpha(\omega)=\frac{2 \omega n^{\prime \prime}(\omega)}{c} \approx \frac{N_{d} e}{n_{b} \epsilon_{0} c} \mu(\omega)
$$

where $n^{\prime \prime}(\omega)$ denotes the imaginary part of the refractive index. A deviation of this relation is given in appendix B.

Measurements of the mid-infrared absorption of n-type gallium arsenide with a doping concentration between $N_{d} \leq 5 \times 10^{14} \mathrm{~cm}^{-3}$ (sample 1) and $N_{d}=5.4 \times 10^{18} \mathrm{~cm}^{-3}$ (sample 6) are shown in Figure 2.11 [68]. The absorption coefficient shows an $\omega^{-3}$-frequency dependence, which is in contrast to the frequency dependence resulting from the Drude model.At mid-infrared frequencies, electrons are accelerated on time scales comparable to the lattice response time $(\omega / 2 \pi)^{-1}$. Therefore, the approximations of equation (2.18) are violated and the Boltzmann transport theory makes incorrect predictions. A more accurate description must account for the quantum kinetic nature of the scattering process. The absorption coefficient at mid-infrared frequencies is usually calculated in second-order perturbation theory [69, 70, 71].

\section{Intraband relaxation via scattering}

The gain in kinetic energy of the electrons in an external electric field is transferred to the crystal lattice via scattering. Electron-electron scattering does not lead to an energy dissipation out of the electron ensemble but only leads to a momentum redistribution. The dominant channel for energy relaxation in a material depends on the experimental conditions like the temperature or the carrier concentration. Figure 2.12 shows the calculated contribution to the Hall mobility of different scattering mechanisms in ndoped gallium arsenide [72]. The Hall mobility measures the lateral electron acceleration in a magnetic field via the voltage induced due to the charge separation. The simulated data is compared to experimentally obtained Hall mobilities taken from reference [73]. Figure 2.12 shows that the dominant scattering process in n-type gallium arsenide at temperatures above $100 \mathrm{~K}$ is polar scattering at optical phonons. At very low temperatures, the intraband relaxation is dominated by scattering at ionized impurities. Scattering 


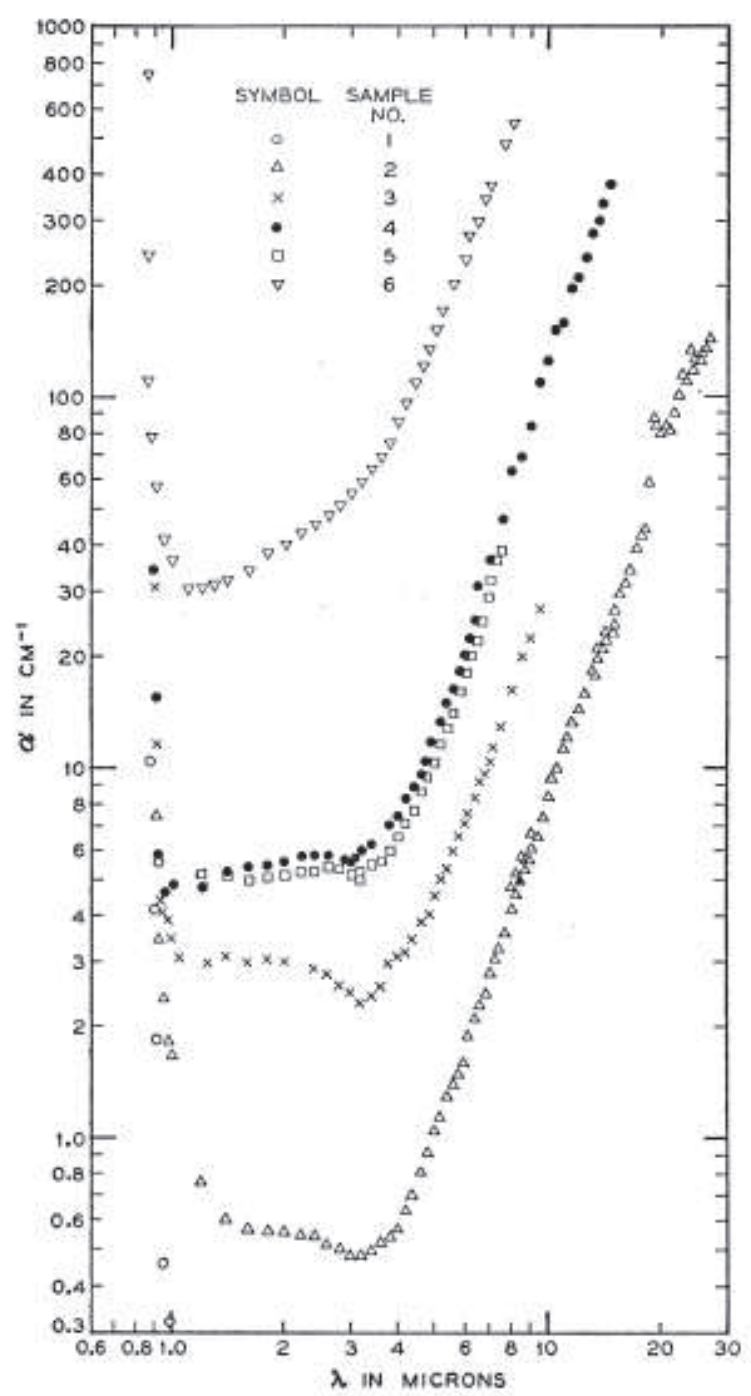

Figure 2.11: Absorption measurement in the mid-infrared range on n-type GaAs with a doping concentration between $N_{d} \leq 5 \times 10^{14} \mathrm{~cm}^{-3}$ and $N_{d}=5.4 \times 10^{18} \mathrm{~cm}^{-3}$ (sample 6). The absorption coefficient shows a $\omega^{-3}$-dependence. The measurement is taken from reference [68]. 


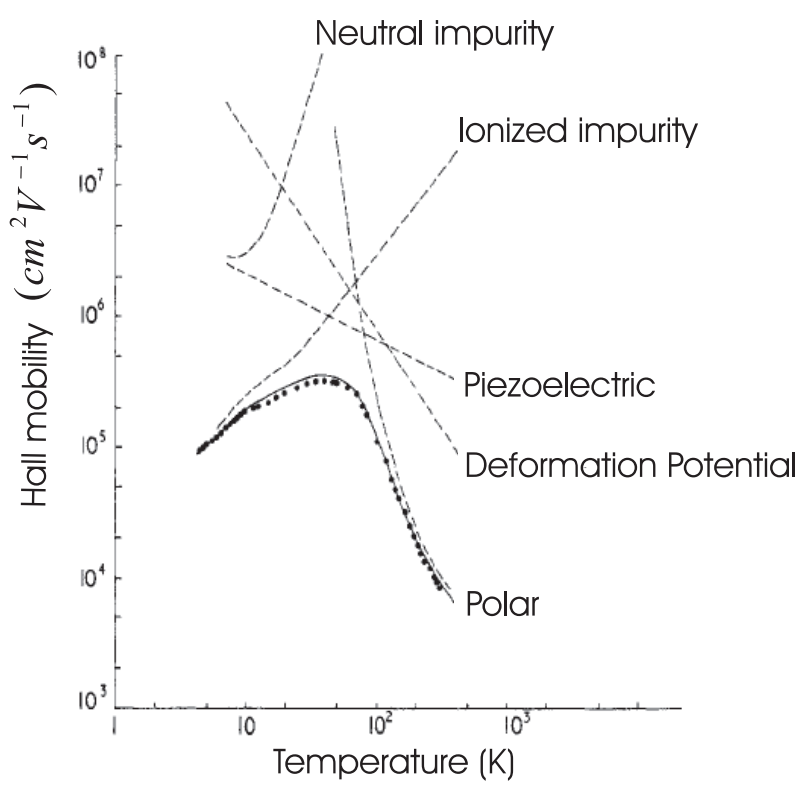

Figure 2.12: Calculated hall mobility of n-type GaAs over temperature (solid line) [72]. Additional experimental data (dotted curve) is taken from reference [73]. The dashed curve shows the contributions of the individual scattering processes. Above $100 \mathrm{~K}$ the mobility is limited by polar optical mode scattering.

at acoustic phonons via deformation potentials [74] or via the piezoelectric interaction $[75,14]$ becomes relevant when the kinetic energy of the electrons is too low to emit optical phonons. Deformation potential scattering at optical phonons $[76,77]$ in gallium arsenide vanishes for symmetry reasons for conduction band electrons at the $\Gamma$-point. Scattering at neutral impurities adds the least contribution to the mobility. The calculated scattering rates are well reproduced in the experimental data. Hence, the transport regime studied here is dominated by polar scattering of electrons at optical phonons.

\subsubsection{Nonlinear electron transport in static external electric fields}

Electrons accelerated in the electric field of an ultrashort $\mathrm{THz}$ pulse gain kinetic energies comparable to the energy of the optical phonon on ultrashort time scales. The approximation of instantaneous scattering, as done in the Boltzmann transport model, is wrong. In addition, at high field strength, the relation between the amplitude of the external field and the electron velocity becomes nonlinear, i.e. Ohm's law is not valid. In contrast a saturation of the velocity is observed. Therefore, in this section, the limits of the BTE are 


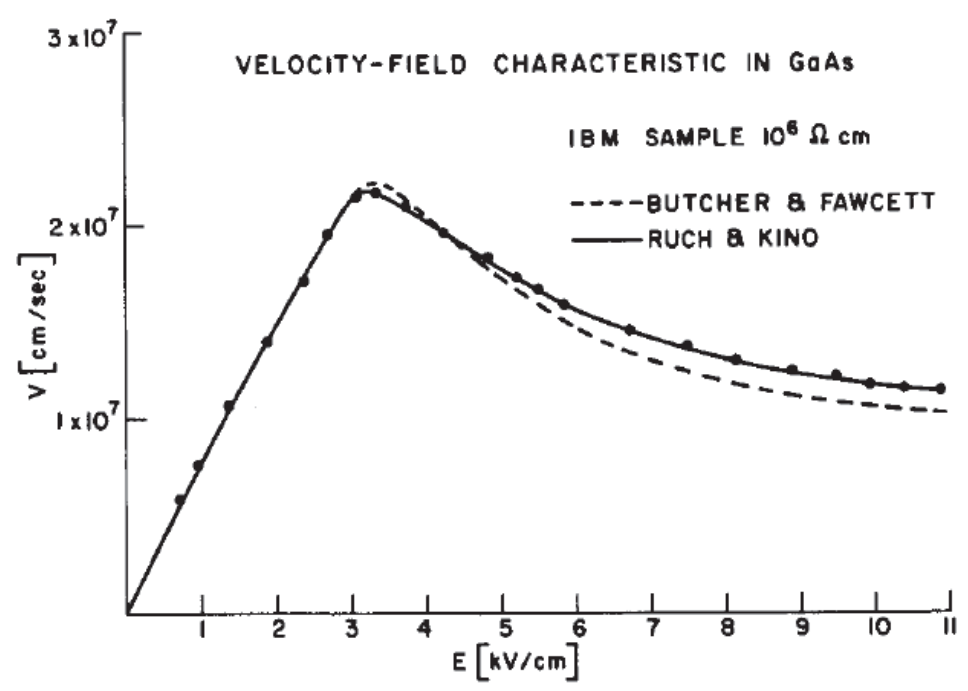

Figure 2.13: Electric field dependence of the drift velocity [78]. For low fields, the velocity increases linearly until it saturates and decreases with rising field strength. This regime corresponds to a negative differential resistance. Experimental data from reference [78] are compared to calculations from Butcher and Fawcett [79]

discussed.

\section{High field electron transport on a long time scale}

In direct bandgap materials like gallium arsenide, indium phosphite, etc., the saturation velocity starts to decrease if the driving field strength is raised, thus leading to a negative differential resistance (NDR). This is shown in Figure 2.13. Note that the velocity dependence on the electric field is depicted for a stationary situation. The effect was first proposed in [80]. It results from scattering of electrons from the $\Gamma$-valley to the L-valley (see Figure 2.1). Due to the smaller curvature of the conduction band in the L- and X-valley compared to the $\Gamma$-valley, an electron in a side valley has a higher effective mass. Therefore it has a lower mobility, which leads to a lower saturation velocity of electrons in the L-valley. Due to the higher density of states in the L-valley, the inter-valley scattering rate for L-valley electrons is lower than in the inverse direction $\tau_{L \rightarrow \Gamma}^{-1}=(2.5 \mathrm{ps})^{-1}<\tau_{\Gamma \leftarrow L}^{-1}<(100 \mathrm{fs})^{-1}[81,82,13]$. For electric fields high enough to promote electrons to the side-valleys by inter-valley scattering, the drift velocity decreases with increasing electric field [83]. However, the dominant scattering process for electrons with small wavevectors in the $\Gamma$-valley is polar optical mode scattering. Therefore, the NDR based on inter-valley scattering is observed in a stationary regime on 
longer time scales, when a significant population in the L-valley is generated. A practical application of this effect is the so-called Gunn-diode [84].

\section{Friction force due to polar optical mode scattering}

The friction force due to polar optical mode scattering can be calculated with the BTE only for low, slowly varying external fields. This restriction results from the Markov approximation in the calculation of the scattering rate. Resonant electron-phonon interaction is expressed by the following condition:

$$
\vec{v}(\vec{k})=\vec{q} \cdot \omega_{L O}
$$

where $\omega_{L O}$ and $\vec{q}$ denote the frequency and the wavevector of the optical phonon, respectively.

The friction force resulting from the Boltzmann transport model in the relaxation time approximation reads:

$$
\vec{F}_{\text {fric }}=\hbar \vec{k} \tau(\vec{k})
$$

where the relaxation time $\tau$ is given by the expression [85]:

$$
\begin{aligned}
\frac{1}{\tau(\vec{k})} & =\frac{2 \pi e^{2} \omega_{L O}}{\hbar \epsilon_{0}\left(\epsilon_{s}-\epsilon_{\infty}\right) \omega}\left\{n\left(1+\frac{\hbar \omega_{L O}}{\epsilon(\vec{k})}\right)^{1 / 2}+(n+1)\left(1-\frac{\hbar \omega_{L O}}{\epsilon(\vec{k})}\right)^{1 / 2}\right. \\
& \left.+\frac{\hbar \omega_{L O}}{\epsilon(\vec{k})}\left[-n \sinh ^{-1}\left(\frac{\hbar \omega_{L O}}{\epsilon(\vec{k})}\right)^{1 / 2}+(n+1) \sinh ^{-1}\left(\frac{\hbar \omega_{L O}}{\epsilon(\vec{k})}-1\right)^{1 / 2}\right]\right\} .
\end{aligned}
$$

Here, $\hbar \omega_{L O}, n, \epsilon_{s}, \epsilon_{\infty}$ and $\epsilon(\vec{k})$ denote the energy of the optical phonon, the material index of refraction, the static and the high frequency dielectric constant and the kinetic energy of the electron, respectively. The friction force $\vec{F}_{\text {fric }}$ is plotted versus the electron velocity in the black line in Figure 2.14. The velocity scale is normalized to the velocity $v_{0}=435.6 \mathrm{~km} / \mathrm{s}$, corresponding to a kinetic energy similar to the energy of an optical phonon. At this point, the friction increases in a step-like fashion and saturates at higher electron velocities. However, if the electron moves faster than the response time of the lattice, i.e. faster than the inverse phonon frequency, it should experience less friction from the lattice. Hence, the Boltzmann transport picture clearly fails at high electron velocities.

A more accurate description of the electron-lattice interaction at high electron velocities is obtained by decomposing the Coulomb field of a classical, point-like electron into the spectral components. The resonant coupling 
to the lattice is introduced via the dielectric function of the medium [86]. The resulting force acting on the electron is given by

$$
\vec{F}_{\text {fric }}^{\text {class }}=\frac{e^{2} \omega_{L O}^{2}}{v(\vec{k})^{2}}\left(\frac{1}{\epsilon_{\infty}}-\frac{1}{\epsilon_{s}}\right) \ln \left(\frac{\vec{k} v(\vec{k})}{\omega_{L O}}\right) .
$$

The classical friction force is plotted in the red line in Figure 2.14. As expected, it decreases for higher electron velocities. However, for low velocities the friction force becomes infinite because a slow electron couples to an infinitely large number of optical phonon modes.

\section{Carrier transport on a short time scale: the quantum kinetic regime}

The transport models discussed so far relied on various assumptions. The BTE describes the electron-phonon interaction in the long-time limit, i.e. an infinite interaction time is assumed. Hence, propagation effects on the time scale of the interaction are neglected. On the other hand, the classical friction force $\vec{F}_{\text {fric }}^{\text {class }}$ assumes point-like charges, which interact with an infinite number of optical phonons. A complete quantum mechanical description must hence account for the finite size of the electron and for the finite interaction time. This so-called short-time limit refers to the quantum kinetic regime [87]. Characteristic quantum features like virtual transitions appear due to timeenergy uncertainty on the time scale of the experiment [88]. In addition, the evolution of the quantum system at time $t$ depends on its history. The dynamics is non-Markovian [89, 90] and exhibits memory effects. Several experimental studies of electron-phonon interaction in the quantum kinetic regime will be briefly discussed in Section 2.4 .3 [91, 92, 93, 88, 94, 95, 96]. The non-Markovian dynamics on short time scales allows for the coherent control of electron-phonon scattering [97, 98].

\section{Final remarks on high field electron transport}

Carrier transport is difficult to characterize over a broad velocity range. The interaction of a moving electron can be described below and above the phonon resonance in independent models. However, both descriptions are unsatisfactory the closer one approaches resonant coupling to the lattice as described by equation (2.29). Figure 2.14 shows the dilemma: While the Boltzmann equation diverges at high velocities (black solid line), the classical model (red solid line) fails to describe the electron-lattice interaction for low electron velocities. The real velocity dependence of the deceleration force on the electron must be a combination of both curves. In particular there should be 


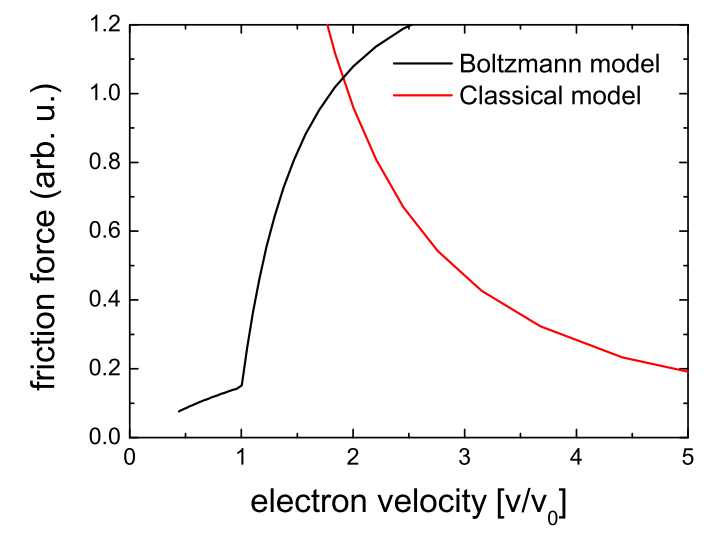

Figure 2.14: Velocity dependent force acting on an electron propagating through a lattice: Boltzmann model (black solid line) and the classical model (red solid line). $\mathrm{v}_{0}=435.6 \mathrm{~km} / \mathrm{s}$ is the threshold velocity for phonon emission.

a saturation velocity from where the deceleration force decreases to higher velocities.

Until now, experimental barriers prevented a deeper investigation of both the linear frequency dependent mobility and the high field transport properties at the lattice resonance. In both regimes the electron-lattice interaction seems to change fundamentally when the phonon barrier is reached. The experiment presented in the second part of this thesis sheds more light on this problem. In addition, a uniform interaction model for linear and nonlinear electron transport is proposed.

\subsection{Electron-phonon interaction}

In this section, polar optical mode scattering is discussed in more details. It is the dominant scattering process which governs the transport behavior in the gallium arsenide lattice in the regime discussed in this thesis.

\subsubsection{Polar optical mode scattering}

An electron propagating through the lattice of a polar host crystal, e.g., gallium arsenide, will lead to a distortion of the latter. The driving mechanism behind the lattice deformation is the Coulomb interaction. While ions with a positive net charge will be attracted by the negative charge of the electron, 

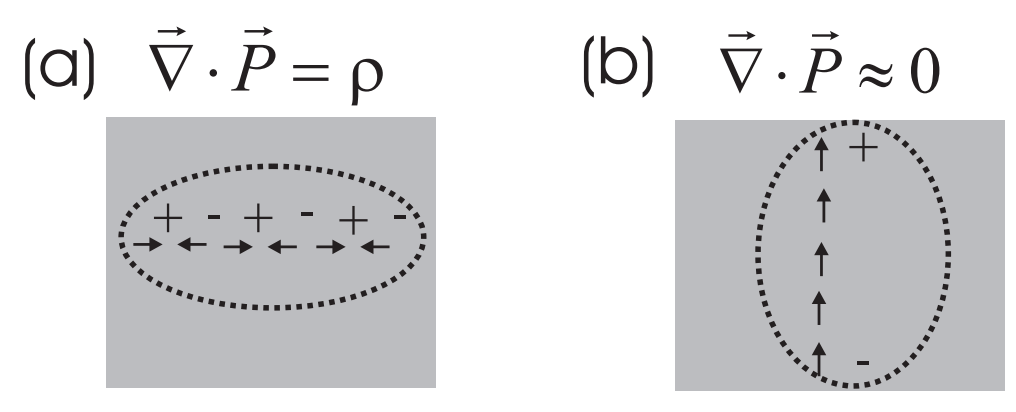

Figure 2.15: (a) Longitudinal optical phonon modes lead to a net space charge density $\varrho$. (b) For transversal modes, a net space charge density exists only at the edges of the mode and vanishes for infinite TEM waves.

negatively charged ions will be repelled. The deformation corresponds to a virtual cloud of longitudinal optical (LO) phonons. The displacement of a lattice ion is given by [85]

$$
\vec{u}(\vec{r})=\sqrt{\frac{\hbar}{2 N M \omega_{L O}}} \sum_{\vec{q}} \frac{\vec{q}}{q}\left(b_{\vec{q}} e^{i \vec{q} \cdot \vec{r}}+b_{\vec{q}}^{\dagger} e^{-i \vec{q} \cdot \vec{r}}\right),
$$

where $b_{\vec{q}}^{\dagger}$ and $b_{\vec{q}}$ are the creation and annihilation operators of the LO-phonon with wave vector $\vec{q}$ and energy $\hbar \omega_{L O}$. The summation stretches over $\vec{q}$ phonon modes, $\mathrm{N}$ depicts the total number of modes and $M=\left(\left(m_{G a}^{-}\right)^{-1}+\left(m_{\overrightarrow{A s}}^{+}\right)^{-1}\right)^{-1}$ is the reduced mass. The displacement $\vec{u}$ leads to a polarization $\vec{P}$ of the lattice, which reads:

$$
\vec{P}=\sqrt{\frac{N M \omega_{L O}}{4 \pi V}\left(\frac{1}{\epsilon_{\infty}}-\frac{1}{\epsilon_{s}}\right)} \times \vec{u}
$$

where $\epsilon_{s}$ and $\epsilon_{\infty}$ describe the static and the high frequency dielectric constant, respectively. In the static case, the polarization includes components from electronic motion and movements of the ions. In the high frequency case, the ions are too slow and $\epsilon_{\infty}$ includes the electronic motion only. $\nabla \cdot \vec{P}$ leads to a space charge density which decomposes uniquely into longitudinal optical modes as shown in Figure 2.15. The electron LO-phonon exchange energy is 
obtained from the induced polarization [99].

$$
\begin{aligned}
H_{e l-p h} & =-e \int d r \frac{\vec{P}(\vec{r}) \cdot\left(\vec{r}-\vec{r}_{e l}\right)}{\left|\vec{r}-\vec{r}_{e l}\right|} \\
& =i \sqrt{\frac{2 \pi e^{2} \hbar \omega_{L O}}{V}\left(\frac{1}{\epsilon_{\infty}}-\frac{1}{\epsilon_{s}}\right)} \sum_{\vec{q}} \frac{\left(b_{\vec{q}} e^{i \vec{q} \cdot \vec{r}_{e l}}-b_{\vec{q}}^{\dagger} e^{\left.-i \vec{q} \cdot \vec{r}_{e l}\right)}\right.}{|\vec{q}|} \\
& =\sqrt{\frac{e^{2}}{V \epsilon_{0}}\left(\frac{1}{\epsilon_{\infty}}-\frac{1}{\epsilon_{s}}\right)} \times \sum_{\vec{q}} \frac{P_{\vec{q}} \cos (\vec{q} \cdot \vec{r})+\omega_{L O} Q_{\vec{q}} \sin (\vec{q} \cdot \vec{r})}{|\vec{q}|}
\end{aligned}
$$

where $\mathrm{r}_{e l}$ denotes the spatial electron coordinate and the phonon coordinate $\mathrm{Q}_{\vec{q}}$ and its conjugate momentum $\mathrm{P}_{\vec{q}}$ have the following relation to the quantum mechanical creation and annihilation operators

$$
\begin{aligned}
\left(b_{\vec{q}}+b_{\vec{q}}^{\dagger}\right) & =\sqrt{\frac{2 \omega_{L O}}{\hbar}} Q_{\vec{q}}, \\
-i\left(b_{\vec{q}}-b_{\vec{q}}^{\dagger}\right) & =\sqrt{\frac{2 \omega_{L O}}{\hbar}} P_{\vec{q}} .
\end{aligned}
$$

$\mathrm{H}_{e l-p h}$ corresponds to the electron-phonon interaction term of the Hamiltonian in equation (2.15).

\subsubsection{The electron in a dressed state: Polaron}

In incoherent electron-phonon interaction processes, like e.g., free-carrier absorption (FCA), a phonon interacts independently with one photon and one electron. The lattice deformation caused by an electron in a polar crystal can in contrast be expressed as a virtual cloud of coherent phonons. Coherent phonons have been reported under various conditions in gallium arsenide and other III-V semiconductor compounds [100, 101, 102, 103]. The interplay of the phonon cloud and the electron forms a quasiparticle [104, 105], which is called polaron [106]. The lattice deformation caused by the electron induces a slight dip in the potential surface leading to a trapping of the electron as shown in Figure 2.16 (a) and (b). The strength of the electron lattice interaction is given through a coupling constant $\alpha_{e p}[107,17]$

$$
\alpha_{e p}=\frac{e^{2}}{\sqrt{2} \hbar \omega_{L O} R_{d}}\left(\frac{1}{\epsilon_{\infty}}-\frac{1}{\epsilon_{s}}\right)
$$

with $R_{d}=\sqrt{\hbar / m^{*} \omega_{L O}}$. The coupling constant can be understood as a measure of the number of LO phonons surrounding the electron. It determines 
(a)

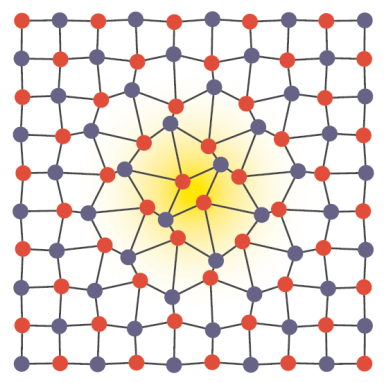

(b)

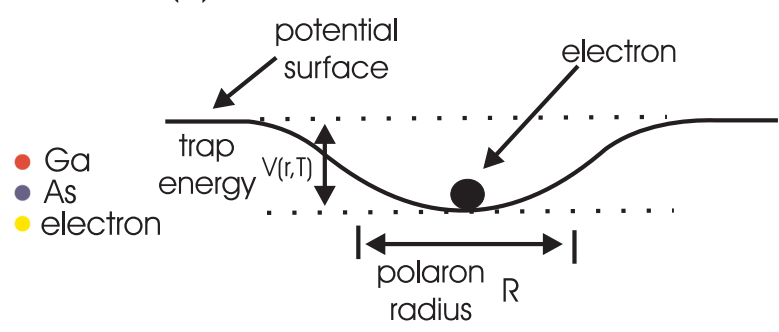

Figure 2.16: (a) Deformation of the gallium arsenide lattice due to a charged electron. The positive As-ions (blue) are attracted and the negative Ga-ions (red) are repelled by the electron. The electron localizes at the gravity center of the potential dip. The ion displacement relative to the equilibrium position is strongly exaggerated. (b) Polaron in the potential sphere. $V(r, T)$ describes the depth of the potential trap and the $\mathrm{R}_{d}$ depicts the radius of the quasiparticle.

the polaron radius $R$, which is defined as the mean-square deviation of the electron from the polaron center

$$
R \approx R_{d} e^{-\frac{k_{B} T}{\hbar \omega_{L O}}}
$$

and the depth of the self-consistent potential trap $\mathrm{V}(\mathrm{r}, \mathrm{T})$

$$
V(r, T)=-\frac{\sqrt{2} \alpha \hbar \omega_{L O}}{r / R_{d}}\left[1-e^{-\sqrt{2 r / R_{d}}}\right]-\frac{\alpha k_{B} T}{2}\left[1+\sqrt{2} r / R_{d}\right] e^{-\sqrt{2} r / R_{d}}
$$

In gallium arsenide one finds $\alpha_{e p}=0.067$ yielding a polaron binding energy of $5 \mathrm{meV}$ and a polaron radius of $\mathrm{R}=2.7 \mathrm{~nm}$ at $\mathrm{T}=300 \mathrm{~K}$ [107].

\subsubsection{Experimental studies of quantum kinetic phe- nomena}

\section{Coherent phonons in gallium arsenide}

Coherent LO phonon oscillations in bulk gallium arsenide are triggered by the space-charge field of photoexcited electron-hole pairs [101, 108]. The electric field associated with the phonon oscillation modulates the linear susceptibility through the first order electro-optic effect. The modulation is monitored with an optical probe pulse. Phonon dephasing times of 4 ps were extracted for moderate excitation densities $\left(\mathrm{N}=10^{17} \mathrm{~cm}^{-3}\right)$. At higher densities, faster dephasing components appear. Time resolved coherent anti-stokes Raman 
(CARS) experiments identified a decay of an LO phonon into a TA phonon and a high wavevector LO phonon at the L-point of the Brillouin zone as the main relaxation channel for the LO phonon [109]. Coherent non-linear $\mathrm{THz}$ spectroscopy on bulk material is a completely new field which is demonstrated here for the first time. However, coherent charge oscillations have been observed in single [110] and double quantum well structures [111] and in semiconductor superlattices [112]. A detailed review on coherent spectroscopy in semiconductors and semiconductor nanostructures is found in $[24]$.

\section{Collective electronic response to an external electric field}

In doped gallium arsenide, an interaction of the plasmon mode of the electron ensemble with the optical modes of the lattice was observed via Raman scattering [113]. The buildup of the plasmon after excitation with an ultrashort femtosecond optical pulse is investigated theoretically in reference [114]. A four-wave mixing experiment allowing to observe the interaction of the plasmon mode with the LO phonon is suggested in reference [115]. In the experiments, a high carrier density is generated with an ultrashort optical pump pulse. The free induction decay shows a superimposed oscillation, where the oscillation period is inversely proportional to the carrier density. This reveals quantum kinetic features of the electron-phonon interaction and the duration of the oscillation corresponds to the interaction memory time. The buildup of a collective electronic response, i.e., a plasmon, is observed experimentally in an optical pump mid-infrared probe experiment in reference [104]. Depending on the pump-probe delay, the complex dielectric function is obtained with a Drude fit. While for short delay times, the mid-infrared response shows only broad structures, a clear plasmon resonance emerges at late delay times. The initial width of the resonance results from the timeenergy uncertainty, due to the finite interaction time of the electron gas. Similar results have been reported on InP [116].

\section{Electron-phonon interaction in the short-time limit}

Early theoretical studies of quantum kinetic transport predict memory effects $[117,118,119]$ and an oscillatory modulation of the polarization decay $[120,121]$. Experimentally, the quantum kinetic regime is difficult to access because it requires a time resolution which is significantly higher than the period of the lattice oscillation ( $\approx 115 \mathrm{fs}$ in gallium arsenide). However, the predicted modulation was observed in 1995 by L. Bányai et al. in a fourwave-mixing (FWM) experiment in undoped bulk gallium arsenide [91]. The 
(a)

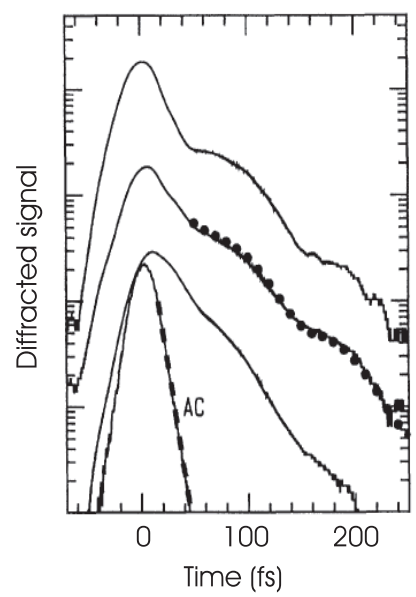

(b)

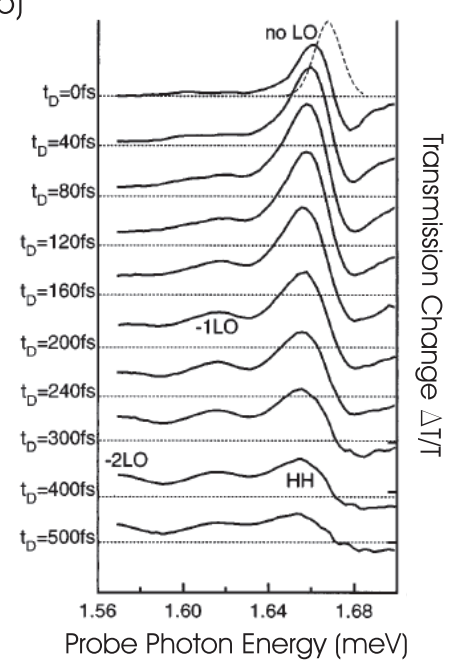

Figure 2.17: (a) Diffracted four-wave mixing signal over time delay for three different carrier densities (from top to bottom: $1.2 \times 10^{16}, 1.9 \times 10^{16}, 6.3 \times 10^{16}$ ). All transients (solid lines) exhibit the same oscillation frequency. The curve labeled $\mathrm{AC}$ is the autocorrelation of the laser pulse, the dotted curve are results from quantum kinetic calculations [91]. (b) Spectrally resolved transmission change $\Delta \mathrm{T} / \mathrm{T}$ in gallium arsenide at a lattice temperature of $15 \mathrm{~K}$ measured for different time delays $\mathrm{t}_{D}$ (solid line). The excitation spectrum is shown as the dashed line. After approximately one phonon oscillation period $(\approx 115 \mathrm{fs})$ a phonon satellite emerged below the principal transmission maximum at approximately $1.65 \mathrm{meV}$. The broad side band at shorter times indicates non-energy conserving scattering events [122].

authors photo-excited electron-hole pairs with a 11 fs optical pulse and observed an oscillation with the frequency $\omega_{\text {osc }}=\left(1+\mathrm{m}_{e} / \mathrm{m}_{h}\right) \omega_{L O}$ independent of the excited carrier density in the diffracted FWM signal. The oscillation results from a beating between various LO-phonon sidebands in the polarization. Results of that experiment are shown in Figure 2.17 (a).

Leitensdorfer et al. [122, 93] observed signatures of the LO-phonon in the time dependent transmittance of a bulk gallium arsenide sample. The authors generated electron-hole pairs by optical excitation and probed the relaxation dynamics with a weak optical probe pulse. The excitation energy is higher then the optical bandgap, which leads to an electron gas with high kinetic energy in the conduction band. The temporal evolution of the transmission spectra reveals the formation of the phonon sidebands as depicted in Figure 2.17 (b). On early time scales, shorter than a LO-phonon oscillation period, the broadening of the phonon sideband points to non-energy con- 


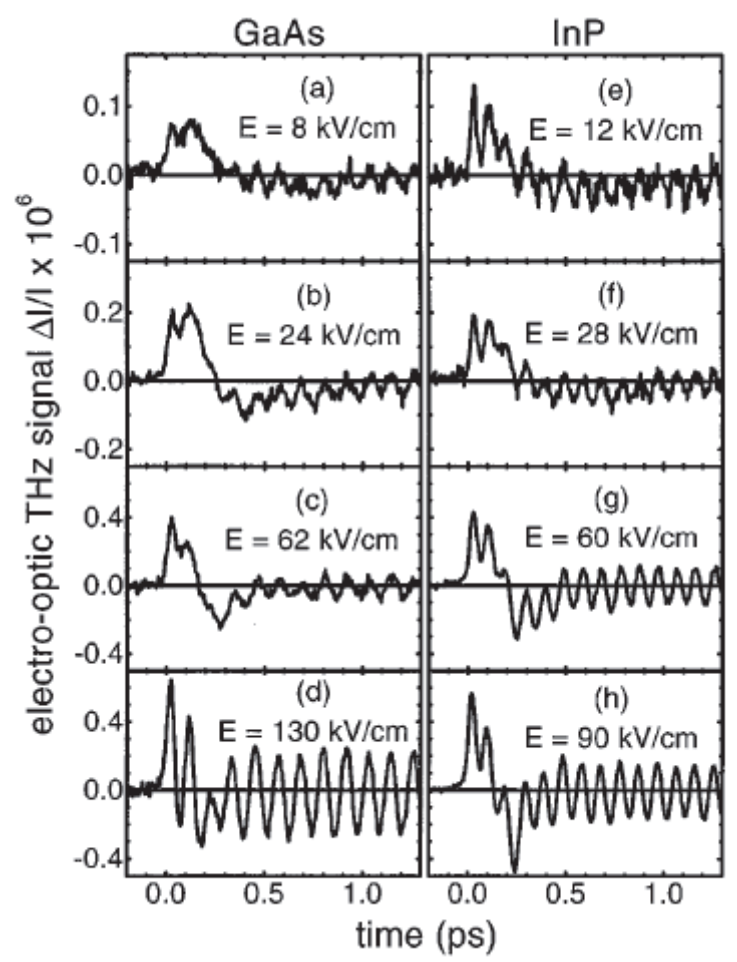

Figure 2.18: $\mathrm{THz}$ transients from GaAs [(a)-(d)] and $\operatorname{InP}[(\mathrm{e})-(\mathrm{h})] p$ - $i$ - $n$ diodes for different bias fields $\mathrm{E}$ at room temperature. The data are taken from reference [100].

serving scattering via virtual transitions as predicted in the quantum kinetic theory. These experiments are particularly interesting because they prove the quantum kinetic nature of the relaxation of a carrier gas with high kinetic excess energy. While the energy is provided during the excitation process of the electron-hole pairs, the application of an ultrashort but intense electric field should create a similar situation.

Photogenerated electrons accelerated by strong electric fields were studied in reference [100]. Results of that experiment are shown in Figure 2.18. The authors used GaAs (Figure 2.18 (a)-(d)) and InP (Figure 2.18 (e)-(h)) $p-i-n$ diodes, respectively, which were biased with a strong electric field at room temperature. After generating photocarriers with an ultrashort optical pulse, the irradiated electric field from the sample was detected using electrooptic sampling (see Section 3.2). The positive slope at early times results from the acceleration of the generated carriers in the electric field. The subsequent negative slope, which emerges for higher bias fields, corresponds to a significant carrier population in the L-valley, where the charges experience 
a lower mobility. At high bias fields, electron scattering from the L-valley to the $\Gamma$-valley becomes resonant to the LO-phonon frequency, triggering coherent phonons, which dominate the observed signal (Figure 2.18 (d) and (h)). The experiment shows that under high electric fields, charge transport is strongly coupled to coherent ionic motions. The high electric field regime will be investigated in a new experiment presented in Chapter 6 with the means of time resolved $\mathrm{THz}$ pump MIR probe spectroscopy.

Due to the non-Markovian dynamics in the quantum kinetic regime, the carrier-phonon interaction can be subject to coherent quantum control $[123,124]$. In a FWM experiment similar to [121], Wehner et al. demonstrated the reversion or the enhancement of a scattering event [97] by introducing a second pump pulse. Variation of the relative delay between the two pump pulses on an attosecond time scale [125] allows for the control of the scattering amplitude. Experiments on the II-VI semiconductors CdSe [96] and ZnSe [95] showed nearly identical results, except that multiple phonon scattering becomes important due to the increased electron-phonon coupling constant $\alpha_{\mathrm{ZnSe}}=0.43$ compared to $\alpha_{\mathrm{GaAs}}=0.065$. Studies in lower dimensional systems have demonstrated that quantum control is feasible for independent scattering mechanisms between a subsystem and different reservoirs [94]. Recently, coherent control of scattering between electrons and acoustic phonons in a gallium arsenide quantum well was demonstrated [98]. An extension of the carrier-phonon quantum kinetics to spatially inhomogeneous systems is presented in reference [126]. 


\section{Chapter 3}

\section{Ultrafast nonlinear spectroscopy in the $\mathrm{THz}$ and mid-infrared range}

This chapter describes the generation and characterization of tunable, intense ultrashort pulses in the mid-infrared (MIR) $(\lambda \approx 9 \mu \mathrm{m}-30 \mu \mathrm{m})$ and $\mathrm{THz}(\lambda \approx 30 \mu \mathrm{m}-300 \mu \mathrm{m})$ range. Such pulses are widely applied in timeresolved studies of a variety of systems, including elementary excitations in semiconductors and semiconductor nanostructures [127], vibrational and rotational molecular spectroscopy [128], imaging [129] and microscopy [130]. The study of coherent phenomena in atoms or semiconductor systems is another important field of application which necessitates intense and reliable sources of few-cycle MIR/THz radiation. In this particular wavelength range the electric field of the radiation can be directly measured in amplitude and phase, thus providing full information on the response of the system under study.

In Section 3.1.1 the laser system used for the generation of the long wavelength radiation is presented. It utilizes a Ti:sapphire oscillator, followed by an amplification and compression stage which yields highly energetic, ultrashort optical pulses.

Section 3.2 gives an introduction in electro-optic sampling which allows to detect the electric field of the $\mathrm{THz} / \mathrm{MIR}$ transients in amplitude and phase. A particular emphasis is laid on the response function of the electro-optic detector.

Section 3.3 describes the generation of MIR radiation via difference frequency mixing and the generation of $\mathrm{THz}$ radiation via optical rectification, as used later in the experimental chapters. In addition, a novel generation mechanism for $\mathrm{THz}$ radiation is introduced, which yields almost single-cycle 
pulses with unprecedented high field amplitudes through four wave rectification in a laser induced plasma. The ability to generate $\mathrm{THz}$ and MIR pulses with high electric field amplitudes has paved the way for nonlinear optics in the $\mathrm{THz}$ regime.

Section 3.4 introduces particular aspects of $\mathrm{THz}$ spectroscopy on samples which are much thinner than the excitation wavelength. There, the carriers responding to the excitation field experience a strong radiative coupling.

Finally, in section 3.5 the experimental setup used in this thesis is presented.

\subsection{Optical pulse generation and shaping}

\subsubsection{Ti:sapphire laser system}

Ultrafast spectroscopy relies on extremely short optical pulses. With the advent of Ti:sapphire lasers, optical pulses with only a few oscillation periods of the electric field have become possible [131]. The main advantage of Ti:sapphire as a gain medium is its large gain bandwidth of about $400 \mathrm{~nm}$ between 670-1070 nm with a maximum around $800 \mathrm{~nm}$ [132], together with its high damage threshold. In addition, it has a high third-order nonlinear coefficient so that ultrashort pulses can be generated via passive Kerr lens mode-locking. Today, pulses with less than 5 fs temporal length can be generated using pulse shaping and compression techniques [133, 134, 135].

The setup employed in this thesis used a Femtolasers Ti:sapphire laser oscillator (Femtosource Scientific Pro) together with a 9 pass chirped pulse amplifier (CPA) system (Femtopower Compact Pro). Figure 3.1 shows a sketch of the oscillator layout.

It is pumped by a frequency doubled $\mathrm{Nd}_{\mathrm{YVO}} \mathrm{YV}_{4}$ laser (Coherent Verdi 5 ) with $4 \mathrm{~W}$ output power at $532 \mathrm{~nm}$. The oscillator generates 12 fs pulses via passive Kerr-lens mode-locking at a repetition frequency of $f_{\text {rep }}=71 \mathrm{MHz}$ and an average output power of $400 \mathrm{~mW}$ which corresponds to a pulse energy of approximately $6 \mathrm{~nJ}$.

After transmission through the output coupler (OC) the beam is divided by two beam splitters (BS). One fraction is guided over a pair of chirped mirrors (CM) which pre-compensate dispersion of the pulse. The main fraction is chirped in a glass block and then spectrally shaped in an acousto-optic modulator (AOM). The glass stretcher reduces the peak intensity of the pulse which otherwise could damage the Ti:sapphire crystal in the amplification stage. The third fraction of the oscillator pulse, which is transmitted through the second beam splitter (BS2), is detected by a photodiode (PD) and con- 


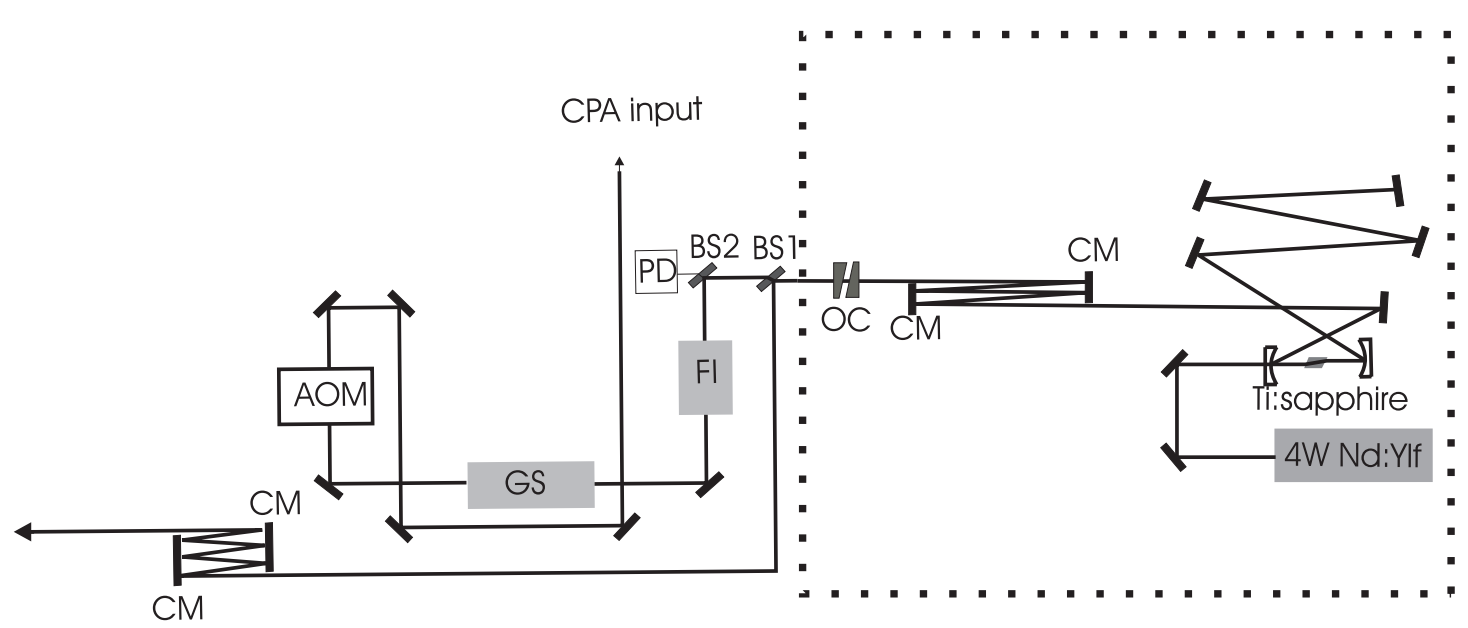

Figure 3.1: Oscillator setup as used in the experiment: The Femtosource Scientific Pro oscillator (framed) yields 12 fs pulses at a repetition rate $f_{\text {rep }}$ of $71 \mathrm{MHz}$, its output power is $400 \mathrm{~mW}$. The abbreviations in the schematic denote: chirped mirror (CM), output coupler (OC), beam splitter (BS), photo diode (PD), Faraday insulator (FI), glass stretcher (GS) and acousto-optic modulator (AOM), respectively.

Table 3.1: Parameters of the oscillator / multipass amplifier laser system

\begin{tabular}{|l|c|c|c|c|}
\hline & pulse length & repetition rate & pulse energy & average output power \\
\hline Oscillator & $12 \mathrm{fs}$ & $71 \mathrm{MHz}$ & $6 \mathrm{~nJ}$ & $400 \mathrm{~mW}$ \\
\hline Amplifier & $25 \mathrm{fs}$ & $1 \mathrm{kHz}$ & $500 \mu \mathrm{J}$ & $500 \mathrm{~mW}$ \\
\hline
\end{tabular}

verted into an electronic pulse train with the oscillator repetition frequency. This signal is used as a synchronization trigger.

The chirped pulse amplifier is shown in Figure 3.2. Stretched pulses from the oscillator are amplified in 9 passes through a Ti:sapphire crystal which is pumped at $532 \mathrm{~nm}$ by a $15 \mathrm{~W}$ frequency doubled Nd:YLF laser with a repetition rate of $1 \mathrm{kHz}$. After the fourth pass the repetition rate is reduced to $1 \mathrm{kHz}$ by a Pockels cell using polarization sensitive optics. Reducing the repetition rate after the fourth pass reduces the amplified spontaneous emission (ASE) per amplified pulse. After the ninth pass the amplified pulses enter the compressor stage which uses glass prisms for chirp compensation. The system yields very intense optical pulses shorter than 30 fs with a pulse energy of approximately $500 \mu \mathrm{J}$.

The main parameters of the laser system are listed in Table 3.1. 


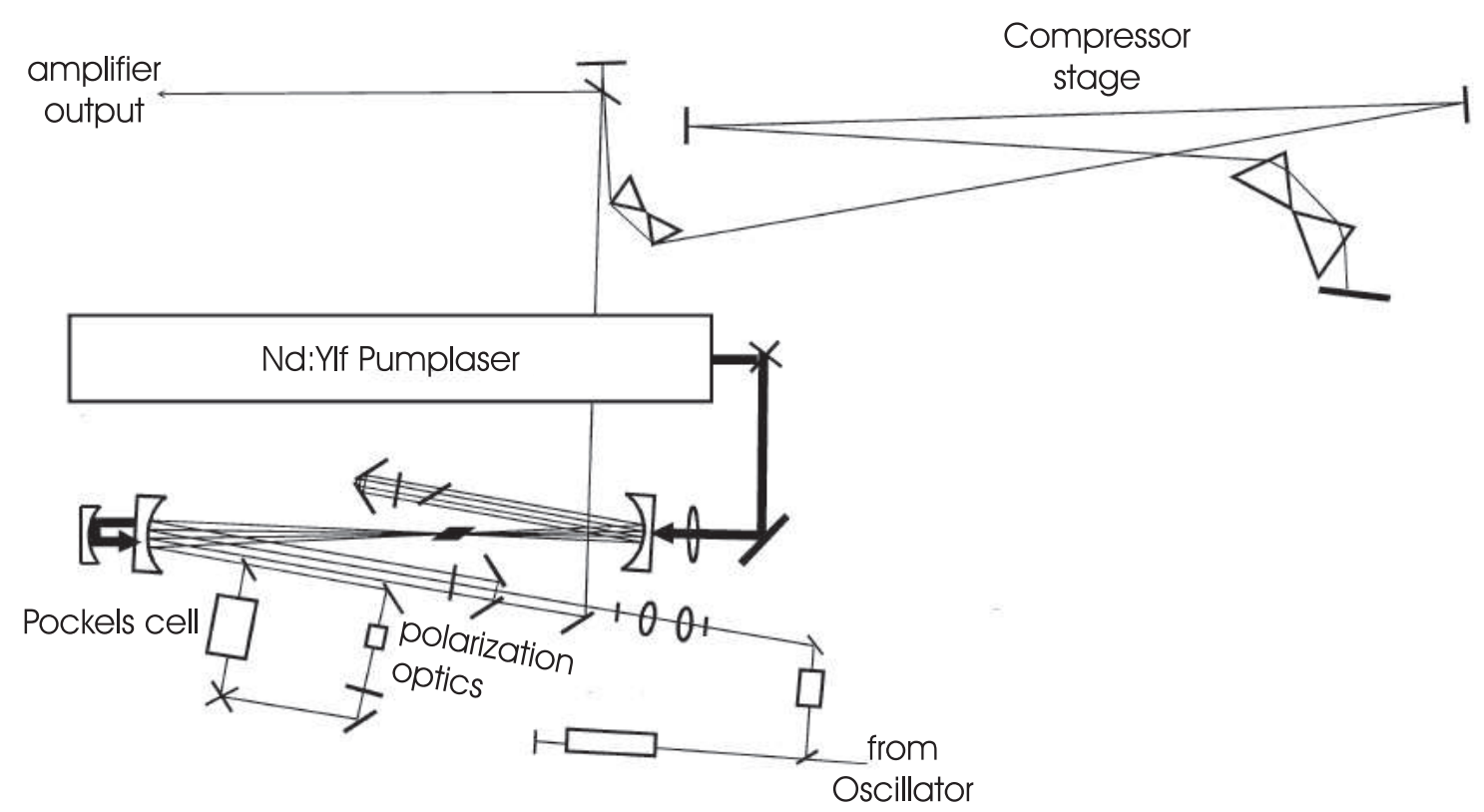

Figure 3.2: Sketch of the Femtopower Compact Pro amplifier [136]: The oscillator pulses are amplified during 9 passes through the Ti:Sapphire crystal which is pumped by a $15 \mathrm{~W} \mathrm{Nd}: Y L F$ laser at $532 \mathrm{~nm}$. After the fourth pass the repetition rate is reduced to $1 \mathrm{kHz}$. The compressor stage compensates for the dispersion of the glass stretcher, reducing the pulse duration to below 30 fs. The pulse energy of the amplified pulses is $500 \mu \mathrm{J}$

\subsection{Characterization of MIR and THz pulses: Electro-optic sampling}

The following section discusses the time-resolved measurement of electromagnetic radiation in the frequency range of 1 to $40 \mathrm{THz}$ via electro-optic sampling (EOS). In a first paragraph the measurement principle is discussed in detail. The following paragraph focuses on the influence of the spatial mapping of the radiation from the source to the detector, which is of particular importance for the $\mathrm{THz}$ range and poses a limit for the sensitivity of the setup for the lowest frequencies. The high frequency limit is determined by the pulse length of the optical probe pulse.

Electro-optic sampling is a powerful technique because it allows for a measurement of the amplitude and phase of the electric field of a $\mathrm{THz}$ or MIR pulse. However, full information on the field can only be gained if the pulses to be measured are synchronized to the optical probe pulses. This case is discussed in the following paragraph. In Appendix $\mathrm{C}$ an experiment is 
presented which addresses specifically the unsynchronized case. Even without synchronization, the autocorrelation spectrum and center frequency can be reconstructed using unsynchronized EOS. At the end of this section, the response function of the EOS detector, which depends on the measurement geometry and on the parameters of the electro-optic crystal is discussed.

\section{The principle of electro-optic sampling}

Electro-optic sampling exploits the second order nonlinear susceptibility $\chi^{(2)}$ of non centro-symmetric crystals to induce birefringence upon exposition to an electric field. This is called the linear electro-optic (EO) effect or, after its discoverer, the Pockels effect [137]. As a result of the induced birefringence, an optical probe pulse suffers a change of its polarization state, which is linear in the electric field applied on the crystal. Figure 3.3 (a) shows how this effect can be exploited to measure MIR or THz transients.

The optical probe pulse polarization is proportional to the electric field of the $\mathrm{THz}$ pulse. The polarization change is detected using a $\lambda / 4$ plate, a Wollaston prism and a pair of balanced photodiodes. Figure 3.3 (b) shows the polarization state at each point in the setup, and Figure 3.3 (c) depicts the sampling principle to record the time dependent THz field (see Figure 3.16).

A popular material for the purpose of EO sampling is ZnTe because the phase velocity of the optical probe pulse matches the group velocity of the $\mathrm{THz}$ pulse. This allows to accumulate the phase shift over the whole crystal length and therefore increases the sensitivity when using thicker crystals. However, the reststrahlen band of $\mathrm{ZnTe}$ between $4 \mathrm{THz}$ and $8 \mathrm{THz}$ [138] prevents a measurement in this frequency range. A higher bandwidth is obtained when using GaP crystals.

For ZnTe crystals (or, in general, crystals having zincblende structure) the $\mathrm{THz}$ field polarization is parallel to the (110) direction, and the probe pulse polarization direction is either (001) or (110). At the end of a crystal of the thickness $d$ this causes a phase difference $\Gamma$ between the polarization components of the optical probe pulse of

$$
\Gamma=\frac{2 \pi d}{\lambda} n^{3}(\lambda) r_{41} E_{T H z},
$$

where $\lambda$ is the vacuum wavelength of the probe pulse, $\mathrm{n}(\lambda)$ is the wavelength dependent refractive index, $d$ is the thickness of the ZnTe-crystal and $\mathrm{r}_{41}$ denotes the nonlinear coefficient. Due to the additional $90^{\circ}$ phase difference added by the $\lambda / 4$ plate, the difference signal of the photodiodes is given by:

$$
\frac{I_{1}-I_{2}}{I_{1}+I_{2}}=\sin (\Gamma) \approx \Gamma .
$$


(a)

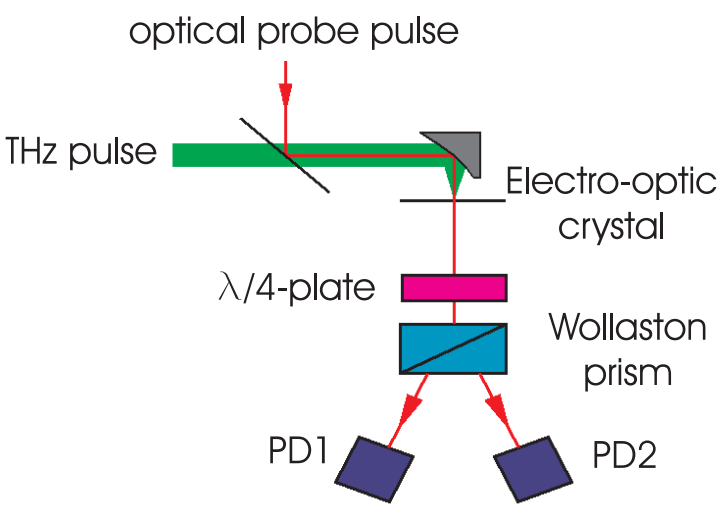

(c)

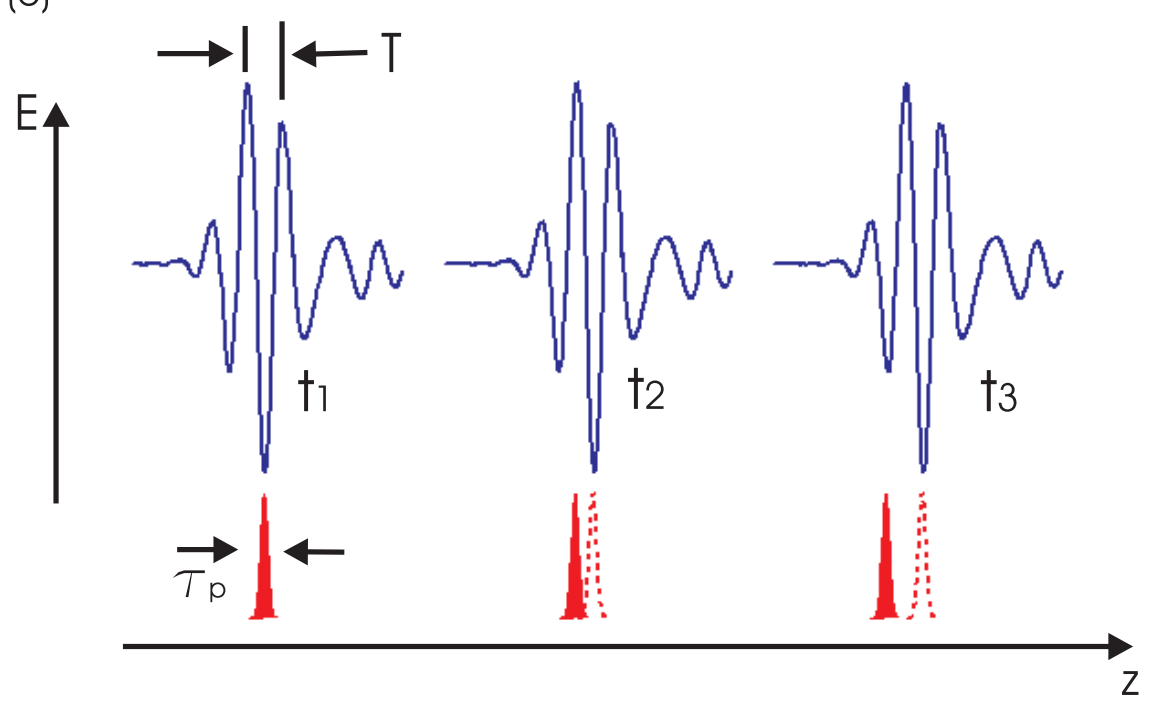

(b)

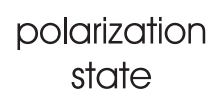

state

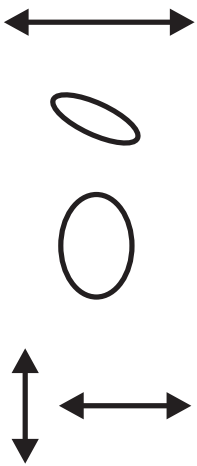
transient: the electric field of the $\mathrm{THz}$ pulse induces birefringence in the $\mathrm{EO}$ crystal, which acts on the linearly polarized optical probe pulse. The polarization change is detected using a $\lambda / 4$ plate, a Wollaston prism and a pair of balanced photodiodes. (b) Polarization state of the probe pulse: linearly polarized before the EO crystal, slightly elliptical after the EO crystal, elliptical after the $\lambda / 4$ plate. The difference between the horizontal and vertical components, i.e., the difference signal of the balanced photodiodes PD1 and PD2, is proportional to the electric field of the $\mathrm{THz}$ pulse. (c) Sampling principle: by delaying the $\mathrm{THz}$ pulse against the optical probe pulse the $\mathrm{THz}$ phase is sampled. To resolve the $\mathrm{THz}$ pulse completely, the duration of the probe pulse $\tau$ has to be shorter than half the period of the $\mathrm{THz}$ pulse: $\tau_{p} \leq \mathrm{T} / 2$. 

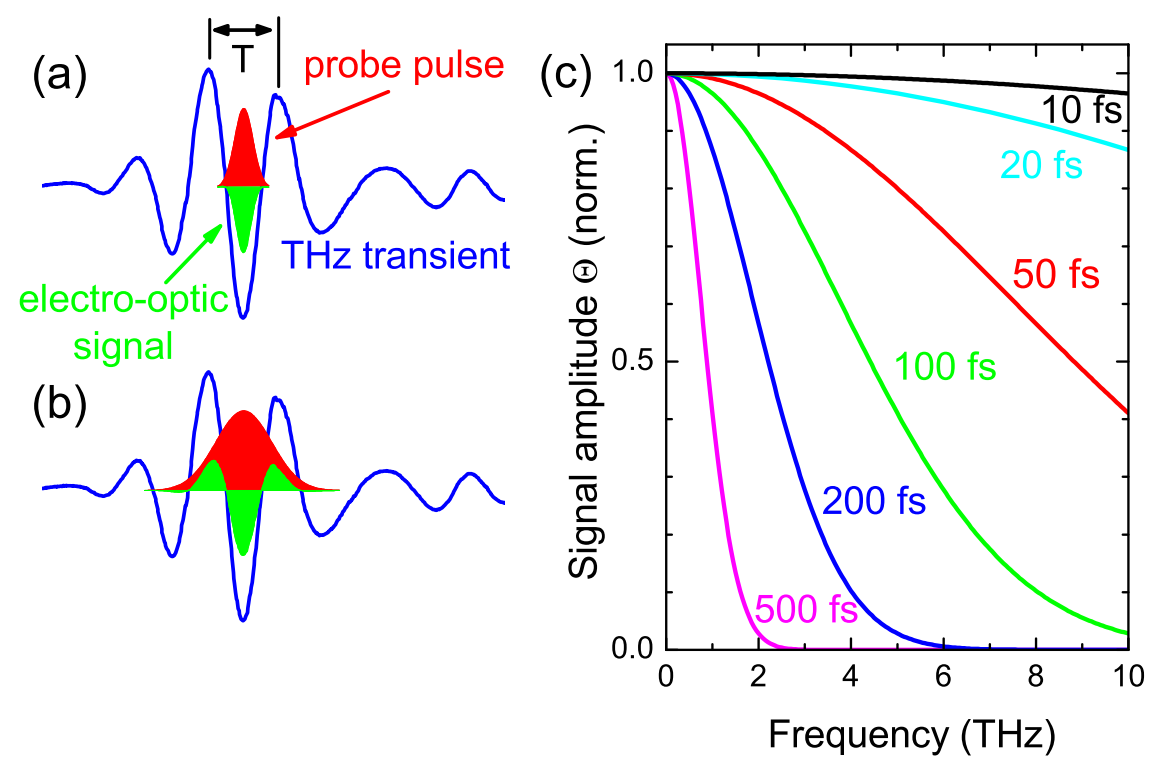

Figure 3.4: (a) THz transient (blue line) sampled by a probe pulse (red) with a length $\tau_{p}=\mathrm{T} / 2$. The electro-optic signal (green) is strictly negative. (b) The same transient sampled by a probe pulse of the length $\tau_{p}>\mathrm{T} / 2$. The elector-optic signal has positive and negative components. (c) Spectral sensitivity of the electro-optic detection for different temporal lengths of the optical probe pulse. The spectral sensitivity is almost flat up to $10 \mathrm{THz}$ for a probe pulse length of $\tau=10 \mathrm{fs}$ (black line). In contrast, for $\tau_{p}=500$ fs probe pulses one can only measure frequencies below $2 \mathrm{THz}$ (pink line). The light blue, red, green and dark blue lines show the spectral sensitivity of the detector for optical probe pulse length of $20 \mathrm{fs}, 50 \mathrm{fs}$, $100 \mathrm{fs}$ and $200 \mathrm{fs}$, respectively.

\subsubsection{Response function of the electro-optic sampling detector}

In the following, the frequency response of the electro-optic sampling (EOS) detector will be discussed. Several design parameters, which enter in the detectable bandwidth, can be influenced by the user, e.g., the length of the optical probe pulse or by the focusing geometry. Other parameters are determined by the materials used, mainly the electro-optic crystal. 


\section{Detection bandwidth and probe pulse length}

The electro-optic signal $\Gamma$ is accumulated over the length of the optical probe pulse. Therefore, the detectable bandwidth is limited by the length of the probe pulse, as shown in Figure 3.4 (a) and (b). In 3.4 (a), the length of the probe pulse (red) $\tau_{p}$ is exactly half the wavelength of the $\mathrm{THz}$ transient (blue line): $\tau_{p}=\mathrm{T} / 2$. This yields an electro-optic signal $\Gamma$ (green), which is strictly negative. The same transient is sampled with a longer probe pulse in Figure 3.4 (b). There, the electro-optic signal has positive and negative components, due to contributions from the positive and negative electric field of the $\mathrm{THz}$ transient. The measured integrated signal is smaller compared to the situation depicted in (a). Thus, the longer probe pulse measures the $\mathrm{THz}$ transient with a lower sensitivity. Frequencies where the contribution of the positive and the negative field are equal cannot be detected. As a rule of thumb, the probe pulse should be shorter than or equal to the half of the period of the maximum signal frequency: $\tau_{p} \leq \mathrm{T} / 2$. This result is extracted from the simulation shown in Figure 3.4 (c). Here, a monochromatic wave is sampled by a gaussian-shaped probe pulse. The signal amplitude $\Theta$ is given by

$$
\Theta=\int_{-\infty}^{\infty} \cos \left(\omega_{0} t\right) e^{-\left(\frac{t}{\sigma}\right)^{2}} d t
$$

where $\omega_{0} / 2 \pi$ denotes the frequency of the monochromatic wave and $\sigma$ denotes the width of the gaussian-shaped probe pulse. $\Theta$ is plotted in Figure 3.4 (c) over the frequency $\omega_{0}$ for different widths $\sigma$ of the probe pulse. For $\sigma=10 \mathrm{fs}$ (black line), the amplitude spectrum is almost flat up to a frequency of $10 \mathrm{THz}$. For a growing pulse width up to $\sigma=500 \mathrm{fs}$ (pink line), the detection bandwidth decreases to approximately $1 \mathrm{THz}$. Intermediate pulse widths are also shown in Figure 3.4 (c).

\section{Spatial mapping of a gaussian shaped $\mathrm{THz}$ pulse in a focusing ge- ometry}

Measurements of the $\mathrm{THz}$ pulse with a knife edge technique as well as measurements of the beam profile carried out by other groups [139] suggest that the $\mathrm{THz}$ beam has a gaussian spatial profile. A discussion on gaussian beams can be found in many text books. This section follows basically [25].

The simulated setup is shown in Figure 3.5 (c). The THz pulse which is emitted from a point source (see Section 3.3.2) is collimated by a $f=25 \mathrm{~mm}$ parabolic mirror with a high F-number and then refocused by a similar mirror on the ZnTe crystal. The short focal length leads to a tight focusing of the $\mathrm{THz}$ beam and hence to high intensities in the focus. The model employed to 


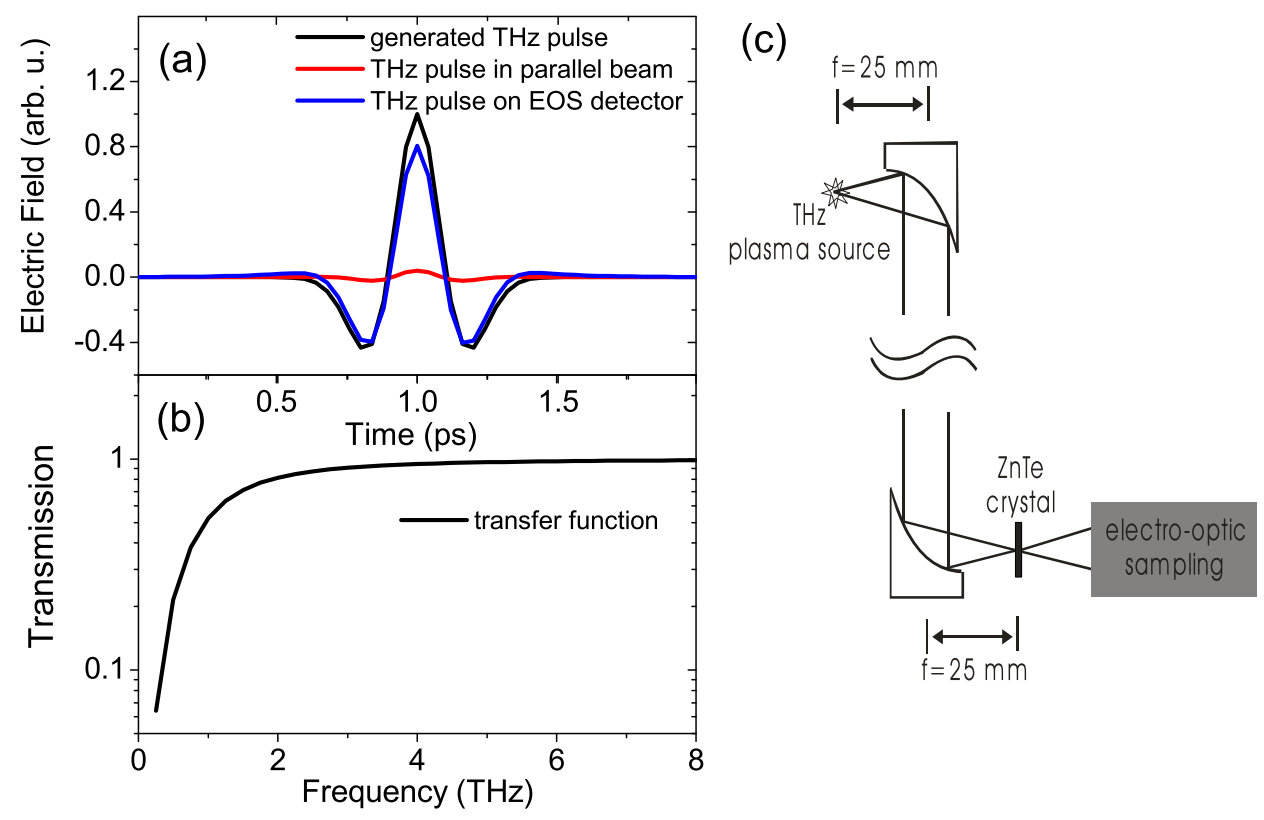

Figure 3.5: (a) Calculated temporal $\mathrm{THz}$ waveforms: generated pulse (black), collimated pulse (red) and pulse focused on the EOS detector (blue). (b) Transfer function of the setup shown in (c). (c) Setup of the simulation: The THz transient is generated in a plasma source (see section 3.3.2), collimated by a $f=25 \mathrm{~mm}$ parabolic mirror and then focused on the EO crystal by a similar parabolic mirror.

calculate the propagation of gaussian beams is described in reference [140]. It accounts for wavelength dependent beam widening and diffraction effects of the propagating pulses. Calculated transients are shown in Figure 3.5 (a) and the transfer function of the setup is shown in 3.5 (b). It is perfectly flat on the high frequency side of the spectrum. The detector looses sensitivity only for very low frequencies. This results from the increasing focal spot size of the $\mathrm{THz}$ beam for longer wavelengths. At $1 \mathrm{THz}$ the transmission has dropped to $50 \%$. Spatial mapping of the radiation is therefore only an issue for frequencies below $1 \mathrm{THz}$.

Another geometric effect on the measured pulse shape is the Gouy phase shift. Because it affects the carrier-envelope phase, it is most relevant for few-cycle or single-cycle pulses. The Gouy shift is a property of any focused wave [141] and results from the transverse confinement which is related to the transverse momentum through the uncertainty principle [142]. A focused 


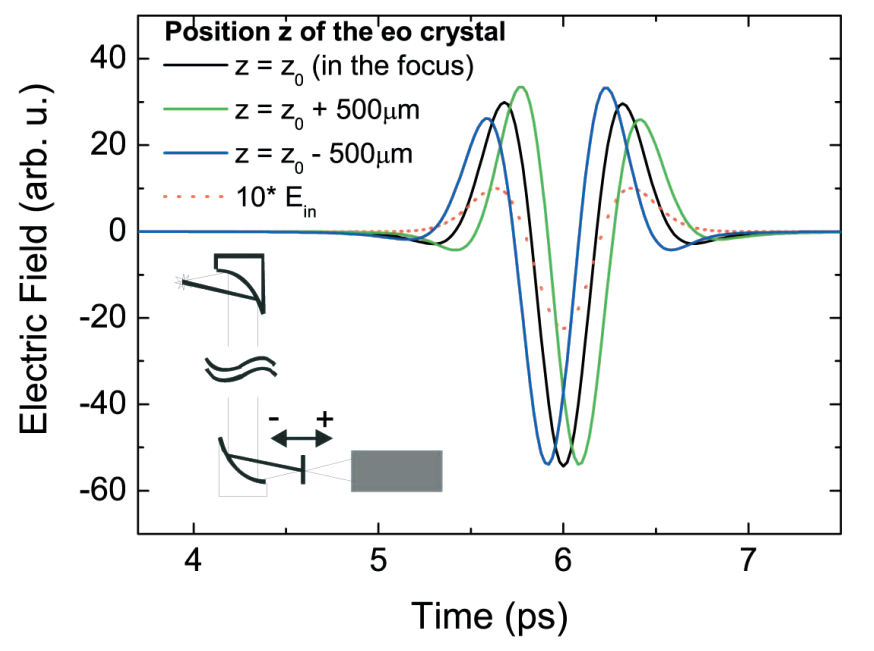

Figure 3.6: Effect of the Gouy phase shift on the pulse shape: translation of the EO crystal along the propagation direction of $500 \mu \mathrm{m}$ leads to a slight asymmetric pulse shape (blue, green) of the single cycle $\mathrm{THz}$ pulse compared to the symmetric pulse obtained for perfect alinement (black). The incident pulse (dotted red) is enlarged. The inset shows the mapping geometry.

gaussian beam suffers a carrier-envelope phase shift of $\pi / 2$ in the focus. Therefore the measured pulse shape can depend on the position of the electrooptic crystal [143, 144].

Figure 3.6 shows the effect of a misaligned EO crystal on the pulse shape. The deviation of the central focus position was taken to be $500 \mu \mathrm{m}$, which is more than the thickness of the crystal in our measurements. One observes a slight deviation of the pulse shape compared to the pulse measured at the optimal position. However, a transversal shift of the crystal of $500 \mu \mathrm{m}$ probably exaggerates the misalignment of the crystal compared to a real measurement. Therefore a significant modification of the measured pulse shape by the Gouy shift is not expected.

\section{Frequency dependence of the electro-optic coefficient $\mathbf{r}_{41}$}

The electro-optic coefficient is composed of an electronic and an ionic response of the material. The ionic response is governed by the resonance of the TO phonon. The ratio between the electronic and the ionic contribution to the electro-optic coefficient is expressed in the Faust-Henry coefficient 
Table 3.2: Parameters for the calculation of the transfer function of a ZnTe electro optic detector

\begin{tabular}{|c|c|c|c|c|c|c|}
\hline & $\omega_{T O} /(\mathrm{THz})$ & $\omega_{L O} /(\mathrm{THz})$ & $\gamma /(\mathrm{THz})$ & $\epsilon_{\infty}$ & $C$ & $n_{g} @ 835 \mathrm{~nm}$ \\
\hline $\mathrm{ZnTe}$ & 5.306 & 6.176 & 0.09 & 6.7 & -0.07 & 3.224 \\
\hline
\end{tabular}

[145]. The frequency dependent electro-optic coefficient reads [146]:

$$
r_{41}(\omega)=r_{e}\left[1+C\left(1-\frac{\omega^{2}-i \omega \gamma}{\omega_{T O}^{2}}\right)\right] .
$$

$\mathrm{r}_{e}$ is the purely electronic contribution to the nonlinearity, $C$ is the FaustHenry coefficient, $\omega_{T O}$ is the frequency of the TO phonon and $\gamma$ is the TO phonon damping constant. The values of these parameters are listed in Table 3.2. $C$ is negligibly small for ZnTe. Therefore the frequency dependence of $r_{41}$ is very small. However, this does not apply for other materials commonly used for electro-optic sampling, e.g., GaP.

\section{THz-probe pulse interaction in the electro-optic crystal}

The modulation of the probe pulse by the $\mathrm{THz}$ field in the electro-optic crystal depends on the phase matching between the phase velocity of the $\mathrm{THz}$ or MIR pulse and the group velocity of the optical probe pulse:

$$
v_{\mathrm{gr}}\left(\omega_{\mathrm{opt}}\right)=\frac{d \omega}{d k\left(\omega_{\mathrm{opt}}\right)} \stackrel{!}{=} v_{\mathrm{ph}}\left(\omega_{\mathrm{THz}}\right)=\frac{\omega}{k\left(\omega_{\mathrm{THz}}\right)} .
$$

Typically this condition holds only for one particular frequency and introduces a frequency dependent detection sensitivity. In addition the probe pulse modulation is also influenced by reflections at the crystal facets and absorption inside the crystal. These effects alter the measured $\mathrm{THz}$ or midinfrared pulse form. Such deformations are expressed in a transfer function $\mathrm{G}(\omega)$, which yields the measured signal of an spectrally infinite broad incident pulse.

The complex refractive index of $\mathrm{ZnTe}$ is obtained through a harmonic oscillator model:

$$
n(\omega)=\sqrt{\left[1+\frac{\omega_{L O}^{2}-\omega_{T O}^{2}}{\omega_{L O}^{2}-\omega^{2}-i \gamma \omega}\right] \epsilon_{\infty} .}
$$

With equation (3.6), one obtains a phase matching frequency in ZnTe of $\omega_{\mathrm{pm}} / 2 \pi=3.1 \mathrm{THz}$. For lower frequencies, $\mathrm{n}(\omega)$ is almost flat and the phase mismatch is very low. Hence, the interaction length between the $\mathrm{THz}$ and 
optical pulse can be increased by using thicker crystals, which leads to a higher sensitivity in the $\mathrm{THz}$ range.

Reflection, absorption and the velocity mismatch are accounted for in the following transfer function [147]:

$$
G(\omega)=\frac{2}{(n(\omega)+1) \delta(\omega)} \int_{0}^{\delta(\omega)} d t e^{i 2 \pi \omega t}=\frac{2}{(n(\omega)+1) \delta(\omega)} \frac{e^{i 2 \pi \omega \delta(\omega)}-1}{i 2 \pi \omega},
$$

where

$$
\delta(\omega)=\frac{n_{g r}-n(\omega)}{c} d
$$

$n_{g r}=\mathrm{v}_{\mathrm{gr}} c^{-1}$ denotes the optical group index, $n(\omega)$ is the THz or MIR phase index, $d$ is the crystal thickness and $c$ denotes the vacuum velocity of light. The parameters of $\mathrm{G}(\omega)$ for $\mathrm{ZnTe}$ are listed in Table 3.2.

\section{Transfer function of electro-optic sampling in $\mathrm{ZnTe}$}

The transfer function of electro-optic sampling in ZnTe is obtained by combining the results of the previous paragraphs:

$$
R(\omega)=G(\omega) \times r_{41}(\omega) .
$$

It is plotted in Figure 3.7. The transfer function depends strongly on the thickness of the electro-optic crystal. For thin crystals $\left(d_{\mathrm{ZnTe}}=10 \mu \mathrm{m}\right)$ the transfer function is almost flat over the whole frequency range, except at the phonon resonance (black solid line). Below the phonon resonance, the sensitivity can be increased by using thicker crystals (red solid line). However, in the mid-infrared range the transfer function calculated with equation (3.7) shows deep spectral fringes which appear above the phonon resonance. These features result from the assumption that the measured pulse propagates collinearly with the probe pulse through the electro-optic crystal. In consequence, the time integral in equation (3.7) stretches over a rectangular window, which is determined by the propagation time in the crystal. In the Fourier domain the rectangular window corresponds to the sinc-function

$$
\operatorname{sinc}(x)=\frac{\sin (x)}{x}
$$

resulting in the spectral fringes. These structures are an artefact of the transfer function and do not appear in the measurement. For short crystals these artifacts are negligible, because they occur at much higher frequencies. 


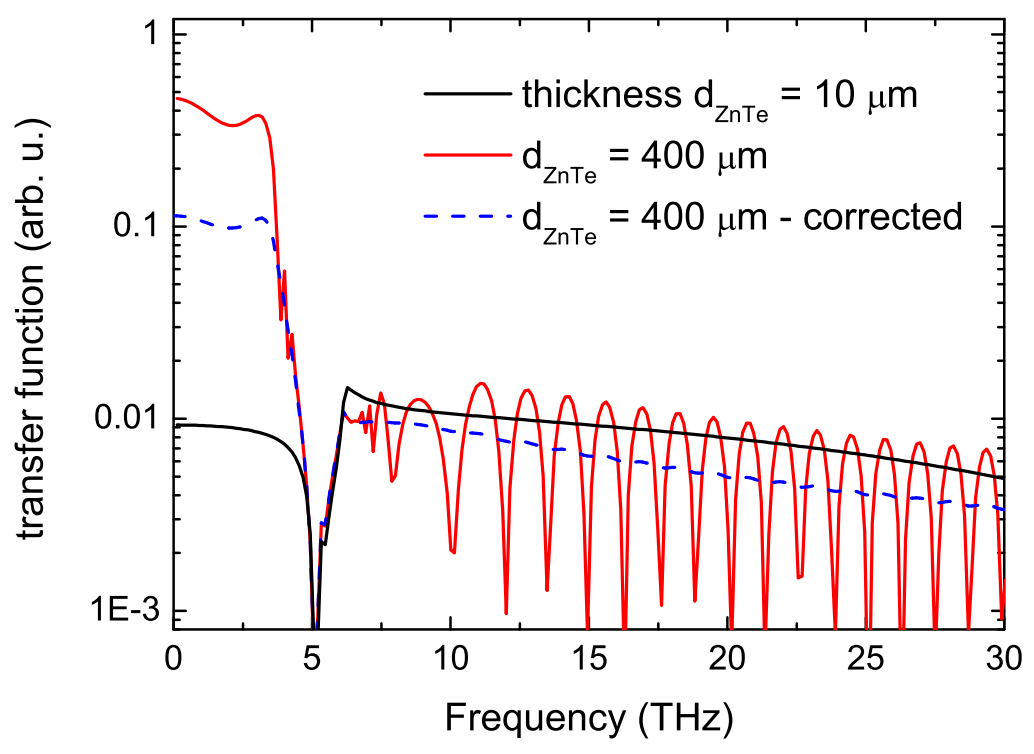

Figure 3.7: Transfer function of the electro-optic detector. It is flat except at the phonon resonance for a thickness $d_{\mathrm{ZnTe}}=10 \mu \mathrm{m}$ of the $\mathrm{ZnTe}$ electro-optic crystal (black solid line). The $\mathrm{THz}$ sensitivity is increased for thicker crystals (red solid line) but shows spectral features due to the focal mismatch between the $\mathrm{THz}$ pulse and the optical probe pulse. A corrected transfer function is shown in the blue dashed line.

However, they become important if an MIR pulse is measured with a thick electro-optic crystal.

A realistic propagation path in a thick electro-optic crystal is depicted in Figure 3.8. Assuming a smooth interaction window, e.g., similar to the gray area, leads to a plane frequency response in the mid-infrared region as shown in the blue dotted line in Figure 3.7. Equation (3.7) must be modified in order to avoid the spurious spectral features by introducing a window function which smoothes the edges of the interaction region. This is achieved by introducing an effective crystal length. In a first step the time integral of equation (3.7) is converted into an integral over the real space coordinate $\mathrm{z}$ :

$$
\int_{0}^{\delta(\omega)} d t e^{i 2 \pi \omega t}=\int_{0}^{d} d z e^{i 2 \pi \frac{z}{\lambda}\left(n_{g}-n(\omega)\right)}=G(d) .
$$

The window function introduced is $\mathrm{e}^{-z / z_{0}}$, where $\mathrm{z}_{0}$ is the effective crystal 


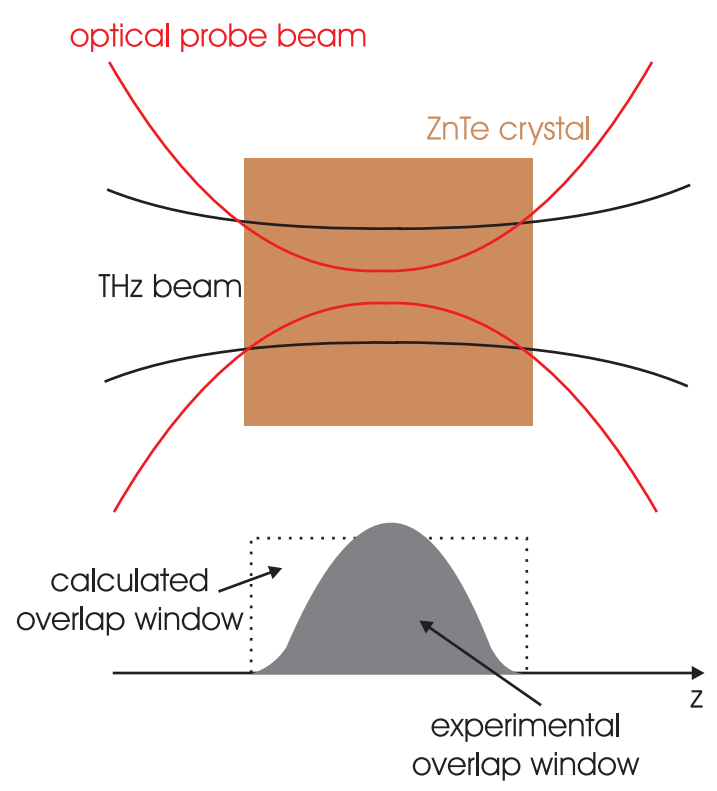

Figure 3.8: Calculation artefact due to the assumption of a parallel propagation if the $\mathrm{THz}$ pulse and the probe pulse in the focus. The assumption leads to a rectangular interaction region (dotted rectangle) which translates into the observed resonance features. The tight focusing in the experiment leads to a beam widening at the at the edges of the ZnTe crystal. The interaction region is therefore smoothed (gray area).

length. It was set to a quarter of the real crystal length, i.e., $\mathrm{z}_{0}=\mathrm{d} / 4$. Equation (3.7) then reads:

$$
G(d)=\int_{0}^{d} d z e^{i 2 \pi \frac{z}{\lambda}\left(n_{g}-n(\omega)\right)} e^{-z / z_{0}} .
$$

Figure 3.7 shows that a $10 \mu \mathrm{m}$ thick ZnTe crystal is an ideal detector for the simultaneous measurement of $\mathrm{THz}$ and mid-infrared radiation due to its flat frequency response. In particular, the calibration to absolute field strengths can be performed at mid-infrared frequencies and assigned to the $\mathrm{THz}$ range. A reliable calibration in the $\mathrm{THz}$ range is more difficult due to the lack of reasonable detectors for $\mathrm{THz}$ radiation. For measurements in the $\mathrm{THz}$ range, thicker electro-optic crystals can be used since they provide a higher sensitivity of the detector.

Experimentally a sensitivity of $1 \mathrm{kV} / \mathrm{cm}$ was found in a single-shot measurement (see Appendix C) for the setup utilized in this thesis. The signalto-noise ratio (SNR) can be increased by averaging over consecutive probe pulses: $\mathrm{SNR} \propto \sqrt{n_{\text {avg }}}$, where $\mathrm{n}_{\text {avg }}$ is the averaging number. The single shot SNR is limited by the shot noise of the optical probe pulses. However, in the 
presented setup the probe pulse energy can not be increased any further due to white light generation in the ZnTe crystal.

The above considerations show that electro-optic sampling is the ideal detection scheme for experiments in the MIR and $\mathrm{THz}$ regime. It is able to detect radiation in the range between $300 \mu \mathrm{m}(1 \mathrm{THz})$ to $9 \mu \mathrm{m}(33 \mathrm{THz})$ or less, depending on the duration of the optical probe pulse. At the same time it is rather insensitive to changes in the beam path geometry, except for extreme situations where the focusing is extremely tight and the pulses are extremely short. The sensitivity can be increased for the $\mathrm{THz}$ pulses by utilizing thick electro-optic crystals for the detection, a measure which does not affect the MIR sensitivity.

Currently extensive research is done in order to find alternative electrooptic detection materials in order to increase the detector bandwidth. An interesting approach is followed by Dai et al. [148], who use a laser-generated plasma for that purpose, thus avoiding perturbing phonon modes. Experimentally the approach of Dai et al. requires additional effort, because a second intense optical pump pulse is necessary for plasma generation. The inverse process, i.e., the generation of $\mathrm{THz}$ radiation in laser-generated plasmas, is discussed in Section 3.3.2. An alternative approach is the use of organic molecules embedded in polymers [149], where the amorphous structure should prevent disturbing phonon oscillations. However, the frequency response of such materials still shows narrow absorption bands, even in the range between $1 \mathrm{THz}$ and $5 \mathrm{THz}$. In addition, the detection sensitivity is lower than that of the standard electro-optic materials like ZnTe and GaP [150].

\subsection{Pulse generation at $\mathrm{THz}$ and mid-infrared frequencies}

\subsubsection{Nonlinear optics and frequency mixing}

The polarization induced in a medium by an incident electric field can be expanded in a Taylor series, which includes the nonlinear components of the susceptibility $\chi$ :

$$
\vec{P}(t)=\underbrace{\epsilon_{0} \chi^{(1)} \vec{E}(t)}_{\text {linear }}+\underbrace{\epsilon_{0} \chi^{(2)} \vec{E}(t)^{2}+\epsilon_{0} \chi^{(3)} \vec{E}(t)^{3}+\cdots+\epsilon_{0} \chi^{(n)} \vec{E}(t)^{n}}_{\text {nonlinear }} .
$$

In this case $\chi^{(n)}$ is described by a tensor of the rank $(n+1)$. Equation (3.13) is useful to describe nonlinear polarizations for non-resonant electric waves propagating through a medium. 
The second order nonlinear interaction is governed by the $\chi^{(2)}$ component of the $\chi$ tensor. Two superimposed waves $\vec{E}(t)=E_{1} e^{-i \omega_{1} t}+E_{2} e^{-i \omega_{2} t}+$ c.c. propagating through a second-order nonlinear medium yield the nonlinear polarization

$$
\begin{aligned}
\vec{P}^{(2)}(t)= & \epsilon_{0} \chi^{(2)}\left[\vec{E}_{1}^{2} e^{-2 i \omega_{1} t}+\vec{E}_{2}^{2} e^{-2 i \omega_{2} t}+2 \vec{E}_{1} \vec{E}_{2} e^{-i\left(\omega_{1}+\omega_{2}\right) t}\right. \\
& \left.+2 \vec{E}_{1} \vec{E}_{2}^{*} e^{-i\left(\omega_{1}-\omega_{2}\right) t}+\text { c.c. }\right]+\epsilon_{0} \chi^{(2)} 2\left[\vec{E}_{1} \vec{E}_{1}^{*}+\vec{E}_{2} \vec{E}_{2}^{*}\right]
\end{aligned}
$$

where the individual terms denote second harmonic generation (SHG), sum frequency generation (SFG), difference frequency generation (DFG) and optical rectification (OR). In order to account for energy conservation, the relation between the participating frequency components reads:

$$
\omega_{\text {out }}=\omega_{1} \pm \omega_{2}
$$

where the subscript out indicates the generated component. Frequency conversion is most efficient when the phase velocity of the optical pulse matches the phase velocity of the generated frequency component, i.e., the overall momentum of the three frequency components vanishes:

$$
\Delta \vec{k}=\vec{k}_{1}+\vec{k}_{2}+\vec{k}_{\text {out }}=0
$$

Compliance to the last equation is called phase matching.

The next section deals with the generation of radiation in the mid- and far-infrared range via difference frequency generation (DFG) and optical rectification (OR).

\section{Difference-frequency generation and optical rectification in a GaSe crystal}

Due to its broad transmission range $(0.62 \mu \mathrm{m}-20 \mu \mathrm{m}$ [151]), its high damage threshold and its high nonlinear coefficient (54 pm/V) [152], GaSe is an effective medium for the conversion of optical wavelengths to the mid-infrared and far-infrared range. In the optical range, GaSe is transparent for a wavelength of $800 \mathrm{~nm}$, but opaque for the second harmonic. Therefore, the SHG and SFG components, which are also generated, are blocked. Optical pulses provided by a Ti:sapphire oscillator multipass amplifier system working at $1 \mathrm{kHz}$ repetition rate are used as pump pulses (see Table 3.1). The high spectral bandwidth $(\approx 48 \mathrm{~nm})$ of the ultrashort pulses allow for the generation of mid-infrared (MIR) pulses between $10 \mathrm{THz}$ and $30 \mathrm{THz}$ by phase-matched type I difference frequency mixing $[153,154]$. In this configuration two optical waves with perpendicular polarization interact, yielding a p-polarized 
(a)

$\mathrm{MgF}_{2}$ polarization
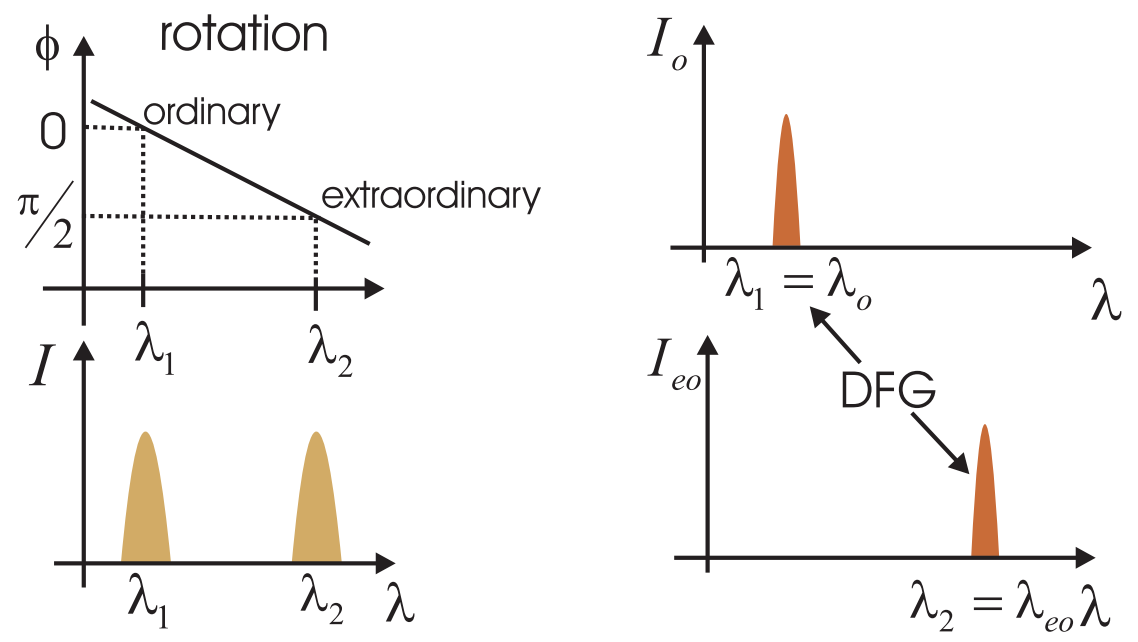

(b)

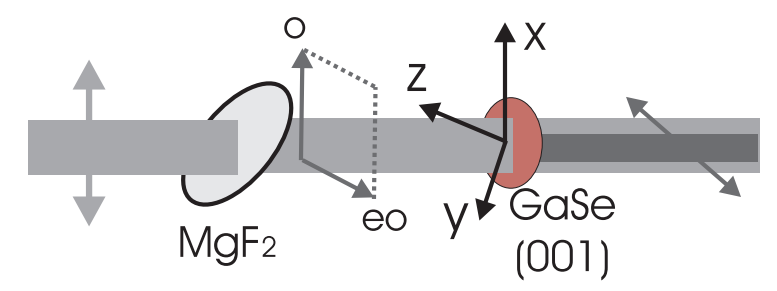

$800 \mathrm{~nm}$

MIR

Figure 3.9: (a) Wavelength selective polarization rotation: The initial intensity spectrum (left side) of the optical pulse contains two peaks at $\lambda_{1}$ and $\lambda_{2}$. The $\mathrm{MgF}_{2}$-plate performs a $90^{\circ}$ rotation of the polarization of the spectral components at $\lambda_{2}$ and conserves the polarization of the $\lambda_{1}$ component. Spectra after propagation through the $\mathrm{MgF}_{2}$-plate (right side) for ordinary $\left(\mathrm{I}_{o}\right)$ and extraordinary $\left(\mathrm{I}_{e o}\right)$ polarizations. DFG occurs between the red spectral components. (b) DFG setup: The vertical polarization of the incident optical pulse is split in a ordinary and extra-ordinary component. MIR pulses with horizontal polarization are generated through type I difference frequency mixing in the (001)-cut GaSe crystal. 

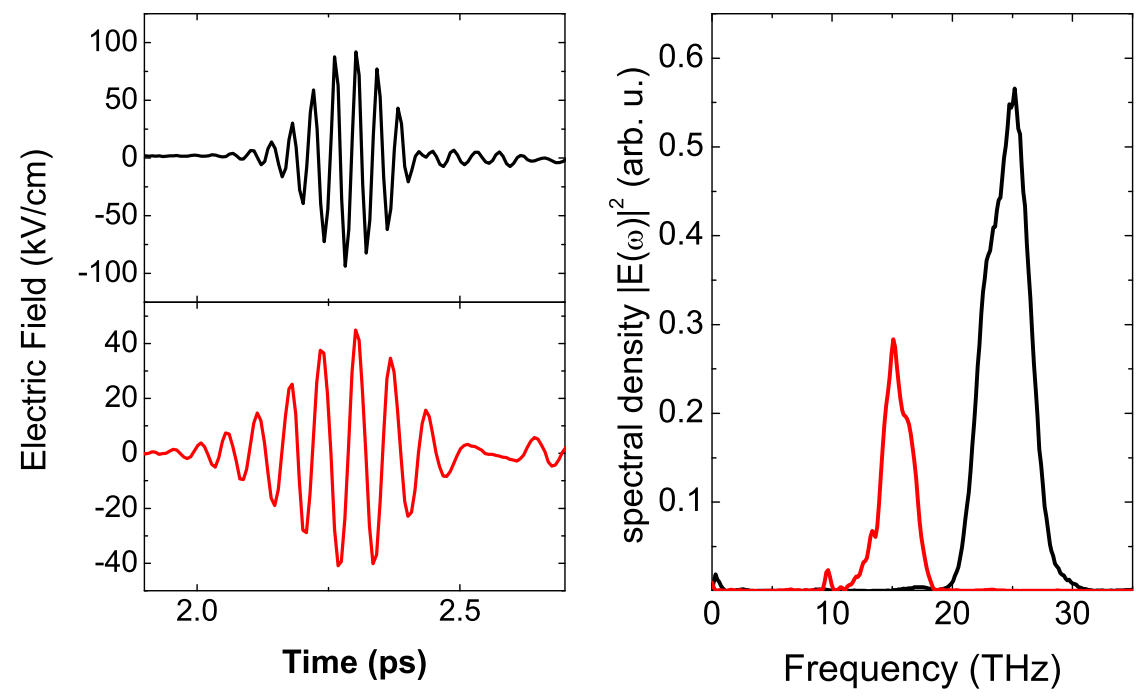

Figure 3.10: MIR pulses generated via DFG in a GaSe crystal. The generation can be optimized via the optical pulse spectrum and phase matching in the birefringent crystal in order to generate pulses in range from $10 \mathrm{THz}$ to $30 \mathrm{THz}$.

MIR pulse (see Figure 3.9). To achieve phase matching, the crystal has to be rotated around the x-axis, such that the optical pulse travels through the yzplane [155]. Depending on the rotation angle, phase matching is optimized for different MIR frequencies [154]. $\mathrm{A} \mathrm{MgF}_{2}$-plate is used to ensure perpendicular polarization of the spectral components which are involved in the DFG process, as shown in Figure 3.9. The difference of the index of refraction in the ordinary direction $\mathrm{n}_{o}$ and in the extra-ordinary direction $\mathrm{n}_{e o}$ of $\mathrm{MgF}_{2}$ depends on the optical frequency and can be adjusted by tilting the $\mathrm{MgF}_{2^{-}}$ plate against the propagation direction of the optical beam. Ideally, it acts as a $\lambda / 2$-plate on one part of the spectrum $\left(\lambda_{e o}\right.$ in Figure 3.9 (a)), i.e, it rotates the polarization for $90^{\circ}$, while conserving the polarization of the other spectral component involved in the mixing process $\left(\lambda_{o}\right.$ in Figure 3.9 (a)). However, the controlled manipulation of the polarization cannot be achieved over the whole bandwidth of the pulse. DFG occurs between the spectra polarized in ordinary and extra-ordinary direction. These spectral regions are narrower than the initial pulse spectra due to the wavelength selective polarization rotation. Figure 3.9 (b) depicts the experimental MIR generation setup. Examples of generated MIR pulses are shown in Figure 3.10. The DFG process can be optimized by manipulating the optical pulse spec- 

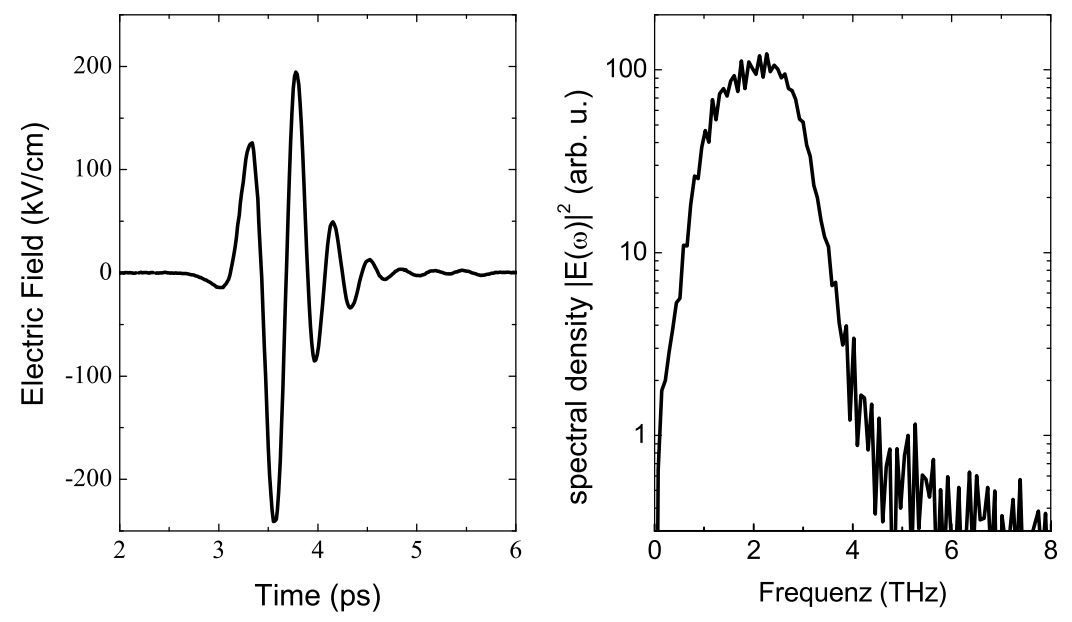

Figure 3.11: THz pulse generated via optical rectification in a GaSe crystal. Electric field amplitudes of $200 \mathrm{kV} / \mathrm{cm}$ can be easily achieved with strong ultrashort optical pump pulses. The pulse spectrum stretches from $1 \mathrm{THz}$ to $5 \mathrm{THz}$, where the detection is screened by the TO phonon in the ZnTe electro-optic crystal.

tra with the acousto-optic modulator and by tilting the GaSe crystal against the propagation direction of the optical beam, in order to generate radiation at different wavelengths. In Figure 3.10, the generation of a MIR pulse at a frequency of $25 \mathrm{THz}(\lambda=12 \mu \mathrm{m})$ (black line) and at a frequency of $15 \mathrm{THz}$ $(\lambda=20 \mu \mathrm{m})$ (red line) is demonstrated. Depending on the thickness of the GaSe crystal, mid-infrared pulses at a wavelength of $15 \mu \mathrm{m}$ with energies up to $300 \mathrm{~nJ}$ can be generated.

The generation of $\mathrm{THz}$ radiation via optical rectification has been studied using a variety of nonlinear materials [156, 157, 158, 159, 160]. Here, the setup shown in Figure 3.9 (b) was employed with slight modifications for $\mathrm{THz}$ generation. The GaSe crystal was placed under vertical incidence in the optical beam and the $\mathrm{MgF}_{2}$-plate was omitted. The mixing occurs between spectrally narrow components, which prevents a frequency selective polarization rotation. Exemplary pulses are shown in Figure 3.11. Pulse energies of $30 \mathrm{~nJ}$ have been realized via optical rectification in GaSe. However, the generated $\mathrm{THz}$ transients have a pulse length of almost $2 \mathrm{ps}$, which is rather long. 


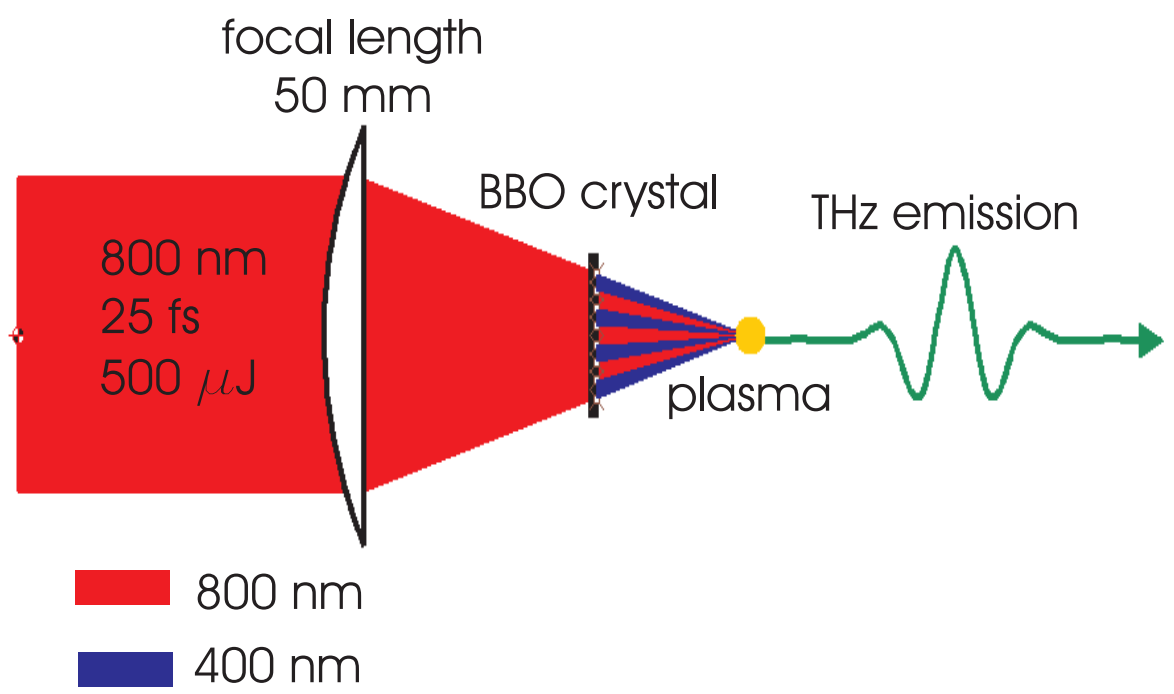

Figure 3.12: Setup for $\mathrm{THz}$ generation: an intense optical pulse $(800 \mathrm{~nm})$ is focused in the surrounding medium, e.g., air. A BBO crystal placed in front of the focus generates second harmonic light by type-I SHG. Due to the high laser intensity in the focus a plasma is generated which acts as a nonlinear medium and leads to the emission of a $\mathrm{THz}$ pulse in a four-wave rectification process.

\subsection{2 $\mathrm{THz}$ pulses generated in laser-induced plasma}

In the last decade, a novel $\mathrm{THz}$ generation mechanism emerged. It is depicted in Figure 3.12. An intense optical pulse $(800 \mathrm{~nm})$ is focused through a $\beta$ barium borate $(\mathrm{BBO})$ crystal in the surrounding medium, e.g., air. In the crystal, second harmonic light is generated via type-I SHG. The high intensity in the focus generates a plasma which acts as a four wave rectification medium for the fundamental and second harmonic pulses.

The first proposal of a $\mathrm{THz}$ source driven by intense, ultrashort optical pulses dates back to 1990 [161]. The authors suggest that the optical pulse focused in air lead to a net space charge, which emits a $\mathrm{THz}$ pulse. An experimental demonstration followed three years later [162]. However, a $\mathrm{THz}$ source as depicted in Figure 3.12 has first been demonstrated by Cook et al. in 2000 [163]. The authors employed 65 fs pulses from a Ti:sapphire laser system with an energy of $150 \mu \mathrm{J}$ at a repetition rate of $1 \mathrm{kHz}$. The energy of the emitted $\mathrm{THz}$ pulse increased by a factor of 4000 compared to a generation with only the fundamental optical frequency as suggested by [161]. The authors suggest a $\chi^{(3)}$ description of the generation process. The inverse mechanism, i.e., THz induced second harmonic generation, has been reported earlier in water and acetonitrile [164], and in silicon [165]. In reference [161] the $\mathrm{THz}$ generation was observed in the $\chi_{i i i i}^{3}$ and $\chi_{i i j j}^{3}$ 


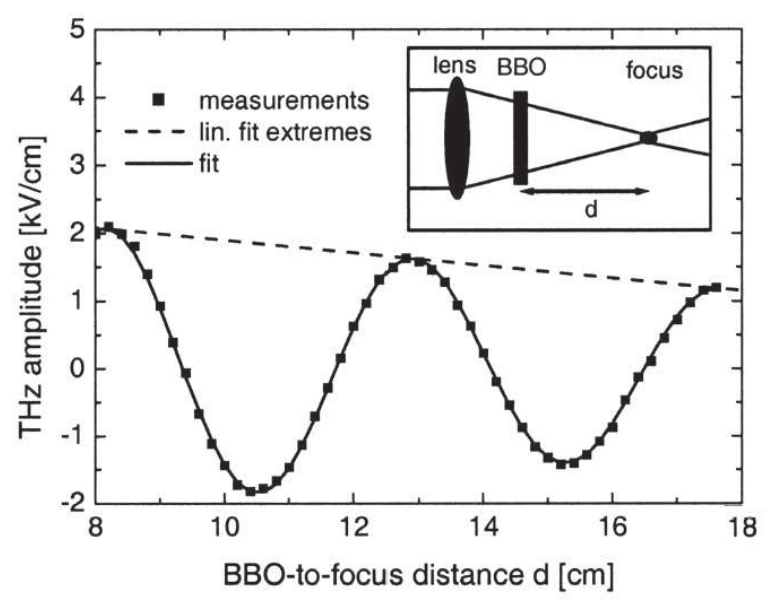

Figure 3.13: Dependance of the $\mathrm{THz}$ amplitude on the distance of the BBO crystal from the focus of a $200 \mathrm{~mm}$ lens (from reference [166]).

configuration. Therefore, the authors suggest that the emission originates from a spatially isotropic medium, e.g., the plasma in the focal region.

Kress et al. showed that $\mathrm{THz}$ radiation in the focus is generated only for intensities higher than the ionization threshold of the medium [166]. Therefore, the plasma is identified as the origin of the nonlinear $\chi^{(3)}$-component. However, the $\mathrm{BBO}$ crystal contributes to the $\mathrm{THz}$ emission via optical rectification already below the plasma threshold. The authors also observe a dependence of the $\mathrm{THz}$ amplitude on the relative phase of the fundamental and second harmonic light shown in Figure 3.13. The relative phase is changed through a variation of the distance of the BBO crystal from the focus, exploiting the group velocity difference between the fundamental and the second harmonic. The observed phase dependence strongly supports a $\chi^{(3)}$-process as the origin of the $\mathrm{THz}$ radiation.

Recently a current surge model has been proposed, which identifies a net dc current in the plasma resulting from an asymmetric laser driving field [167]. The authors measured the phase dependence of the $\mathrm{THz}$ radiation similar to Figure 3.13 and deduce the relative phase of the two optical components by an extrapolation of their data to a BBO-to-focus distance $d=0$. The resulting relative phase allows them to reconstruct the optical field in the focus. Even though such phase dependent currents have been observed in semiconductors [168] and semiconductor nanostructures [169], the approach in reference [167] seems rather questionable because additional arbitrary phase shifts due to plasma nonlinearities [170] should prevent a reconstruction of the optical field in the focus. 


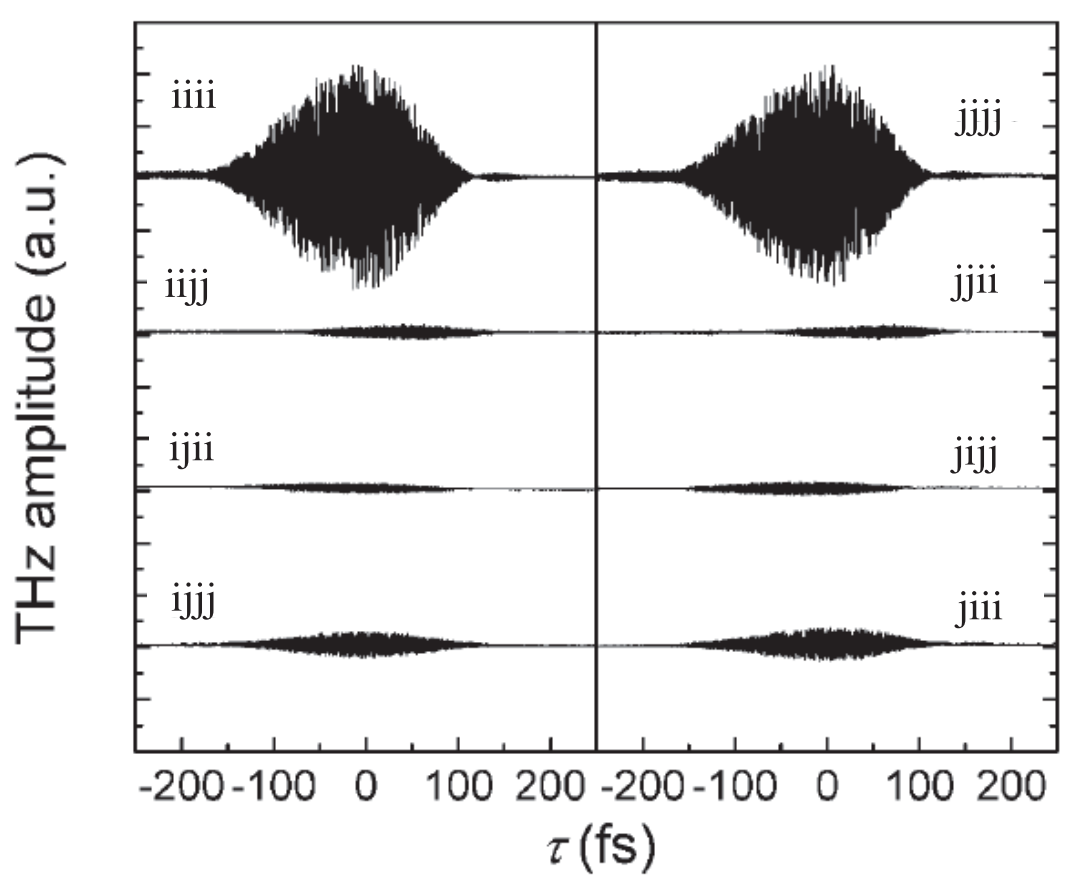

Figure 3.14: $\mathrm{THz}$ amplitude of the four-wave mixing signal of the fundamental and the second harmonic in different components of the $\chi^{(3)}$-tensor. For symmetry reasons the signals on the left and the right side are equal (from reference [174]).

THz generation in ionized gases, e.g., xenon [171], is still a field of active research. A broadband $\mathrm{THz}$ generation and detection setup is presented in reference [172]. The authors use ionized air as generation source and as a detection medium. The generation of radiation via four-wave mixing in ionized gas is extended to the mid-infrared range in referrence [173].

\section{General properties of the plasma nonlinearity}

The third-order nonlinear polarization generated in the focus of the optical pulse reads:

$$
\vec{P}_{i}^{(3)}(t)=\epsilon_{0} \chi_{i j k l}^{(3)} \vec{E}_{2}(t)_{j} \vec{E}_{1}(t)_{k} \vec{E}_{1}(t)_{l} \sin (\varphi),
$$

where $\vec{E}_{1}(t)_{x}$ denotes the electric field at the fundamental frequency of the optical pulse, $\vec{E}_{2}(t)_{x}$ the electric field of the second harmonic and $\varphi$ is the relative phase between the two. Because the second harmonic component is generated by the same optical pulse as the THz pulse, $\vec{P}_{i}^{(3)}(t)$ depends on the fourth power of $\vec{E}_{1}(t)$. 
A detailed study of the plasma nonlinearity was published in 2006 [174]. The authors measured the $\mathrm{THz}$ yield of a laser induced plasma source by individually controlling the fundamental and second harmonic amplitude and polarization as well as the relative phase. The threshold behavior and the power dependence of the $\mathrm{THz}$ signal confirmed previous results. In addition, different $\chi^{(3)}$-components were probed in different polarization configurations, in order to study the symmetry properties of the susceptibility tensor. The results are shown in Figure 3.14.

In a spatially isotropic medium all but the $\chi_{i i i i}^{(3)}, \chi_{i j i j}^{(3)}$ and $\chi_{i i j j}^{(3)}$ vanish. However, in Figure 3.14 also the component $\chi_{j i i i}^{(3)}$ and $\chi_{i j i i}^{(3)}$ yield THz radiation. In contrast the $\mathrm{THz}$ signal generated in the $\chi_{i i j j}^{(3)}$ component is very low. The plasma is therefore not spatially isotropic. The anisotropy may result from the spatial profile of the optical beam, which generates the plasma. This, however, means that the generation conditions depend heavily on the employed optical setup.

A simulation of the generation of $\mathrm{THz}$ radiation through four wave rectification in a $\chi^{(3)}$-medium is depicted in Figure 3.15. The optical pump pulse is composed of the $800 \mathrm{~nm}$ fundamental component $\mathrm{E}_{1}(\mathrm{t})$ and a frequency doubled $400 \mathrm{~nm}$ component $\mathrm{E}_{2}(\mathrm{t})$ with a relative phase shift $\varphi$

$$
E(t)=E_{1}(t)+E_{2}(t) e^{i \varphi}
$$

The superposition signal $\mathrm{E}(\mathrm{t})$ is mixed assuming a nonlinear susceptibility as shown in the inset in Figure 3.15 (d). The calculation was performed at a cubic $\chi^{(3)}$ (black line) susceptibility. In addition, a quadratic $\chi^{(2)}$ susceptibility (red line) is shown. Figure 3.15 (a) and (b) show the superposition signal E(t) (green line) with a relative phase shift of $\varphi=0$ and $\varphi=\pi / 2$, respectively, between the fundamental (black line) and the second harmonic (red line). The calculated nonlinear polarization $\mathrm{P}^{(3)}$ is shown in Figure 3.15 (c) for a relative phase $\varphi=0$ (black line), $\varphi=\pi / 4$ (red line) and $\varphi=\pi / 2$ (blue line), respectively. The inset in Figure 3.15 (c) shows a detailed view on the polarization. The corresponding spectra are shown in Figure 3.15 (d). The maximum spectral density is obtained for a relative phase of $\varphi=\pi / 2$ (blue line). A relative phase of $\varphi=\pi / 4$ (red line) yields less spectral density in the $\mathrm{THz}$ range and a superposition with a relative phase of $\varphi=0$ (black line) does not yield any signal at $\mathrm{THz}$ frequencies. In contrast, mixing at a quadratic $\chi^{(2)}$ susceptibility would yield a phase-independent rectification signal at $\mathrm{THz}$ frequencies. Hence, the observed $\mathrm{THz}$ generation mechanism can be described in a $\chi^{(3)}$-process. A detailed study of $\mathrm{THz}$ generation in laser induced plasmas is given in reference [175] and a detailed review article is found in reference [176]. 

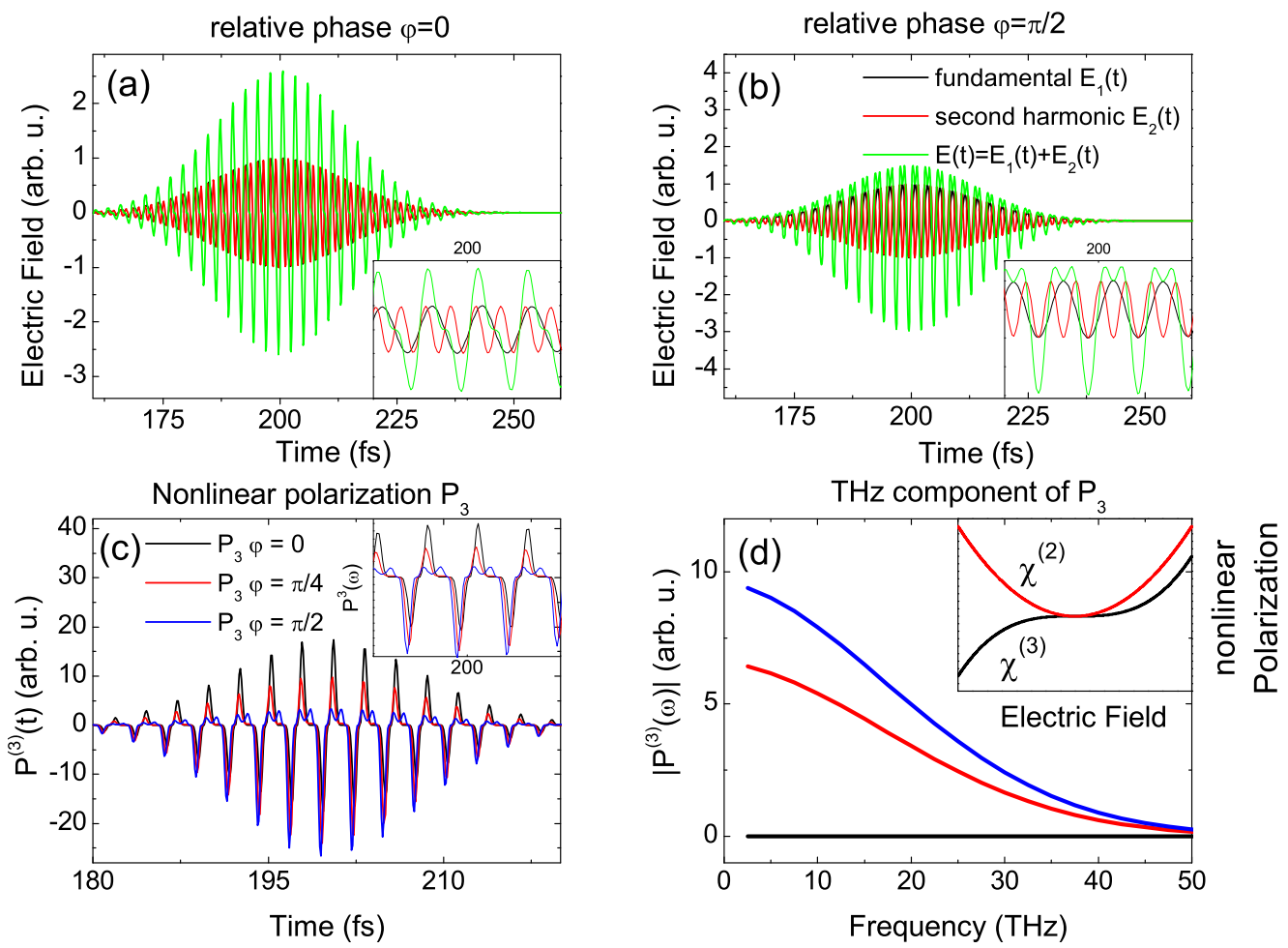

Figure 3.15: (a) Superposition (green line) of the fundamental (black) and the second harmonic light (red line) with a relative phase $\varphi=0$. (b) Superposition with a relative phase $\varphi=\pi / 2$. (c) Nonlinear polarization for different relative phases of the superposition signal: $\varphi=0$ (black line), $\varphi=\pi / 4$ (red line) and $\varphi=\pi / 2$ (blue line). The inset in (a)-(c) shows a detailed view. (d) $\mathrm{THz}$ component of the nonlinear polarization: $\varphi=0$ (black line), $\varphi=\pi / 4$ (red line) and $\varphi=\pi / 2$ (blue line). The insets show the nonlinear susceptibility components $\chi^{(2)}$ (red line) and $\chi^{(3)}$ (black line). 


\section{Generation of ultrashort, intense $\mathrm{THz}$ transients in a nitrogen plasma}

This section presents results of the generation of $\mathrm{THz}$ transients with unprecedented high electric field amplitudes [177] based on four-wave rectification in a laser-generated nitrogen plasma. The generation scheme basically follows Figure 3.12. The optical pulses employed were provided by the Ti:sapphire oscillator/CPA system discussed in Section 3.1.1. The bandwidth-limited optical pulses with a temporal length of $25 \mathrm{fs}$ and a pulse energy of $500 \mu \mathrm{J}$ were focused by a fused-silica lens with a focal length of $50 \mathrm{~mm}$. Without plasma generation one expects a peak intensity in the focus of $10^{17} \mathrm{~W} / \mathrm{cm}^{2}$. A $100 \mu \mathrm{m}$ thick type-I BBO crystal is placed approximately $5 \mathrm{~mm}$ in front of the focal spot for second harmonic generation. THz radiation is generated in the plasma in the focal region through four-wave mixing. The focal length of the lens and the distance of the BBO from the focus have been optimized for maximum $\mathrm{THz}$ signal. Note that this configuration utilizes the $\chi_{i i j j^{-}}^{(3)}$ component of the plasma. The entire setup was purged with dry nitrogen to prevent spurious absorption by water molecules. Generated $\mathrm{THz}$ transients and the corresponding spectra are shown in Figure 3.16 for different lengths of the optical pump pulse.

Transients in Figure 3.16 (a) and (b) were measured in a $0.4 \mathrm{~mm}$ thick ZnTe crystal, the transient shown in Figure 3.16 (c) and (d) was measured using a $0.1 \mathrm{~mm}$ thick GaP crystal as electro-optic detector. The transient shown in Figure 3.16 (c) is the strongest $\mathrm{THz}$ pulse reported to date with a peak amplitude of approximately $450 \mathrm{kV} / \mathrm{cm}$ and a pulse energy of $30 \mathrm{~nJ}$. The short optical pulse length also allows for the generation of $\mathrm{THz}$ transients with an extremely large bandwidth. This is shown in Figure 3.17 (a) and (b). The spectrum of the ultrashort pulse clearly shows the absorption band of the ZnTe detection crystal at $5 \mathrm{THz}$.

For additional measurements, the $\mathrm{THz}$ source was placed in a vacuum chamber to allow for pressure control. In this way $\mathrm{THz}$ radiation generated in the $\mathrm{BBO}$ crystal can be measured without manipulation of the setup [166]. The results are shown in Figure 3.18. The maximum electric field of the transient measured with a pressure of 900 mbar (red line) is $180 \mathrm{kV} / \mathrm{cm}$. Compared to the transient recorded with a pressure of $10^{-3} \mathrm{mbar}$ (black line), where the maximum field is approximately $100 \mathrm{kV} / \mathrm{cm}$, the electric field decreased for about 55\%. In terms of pulse intensity one finds $(100 \mathrm{kV} / \mathrm{cm})^{2} /(180 \mathrm{kV} / \mathrm{cm})^{2}=0.3$, i.e., about $30 \%$ of the power of the $\mathrm{THz}$ pulse is generated in the BBO crystal. 

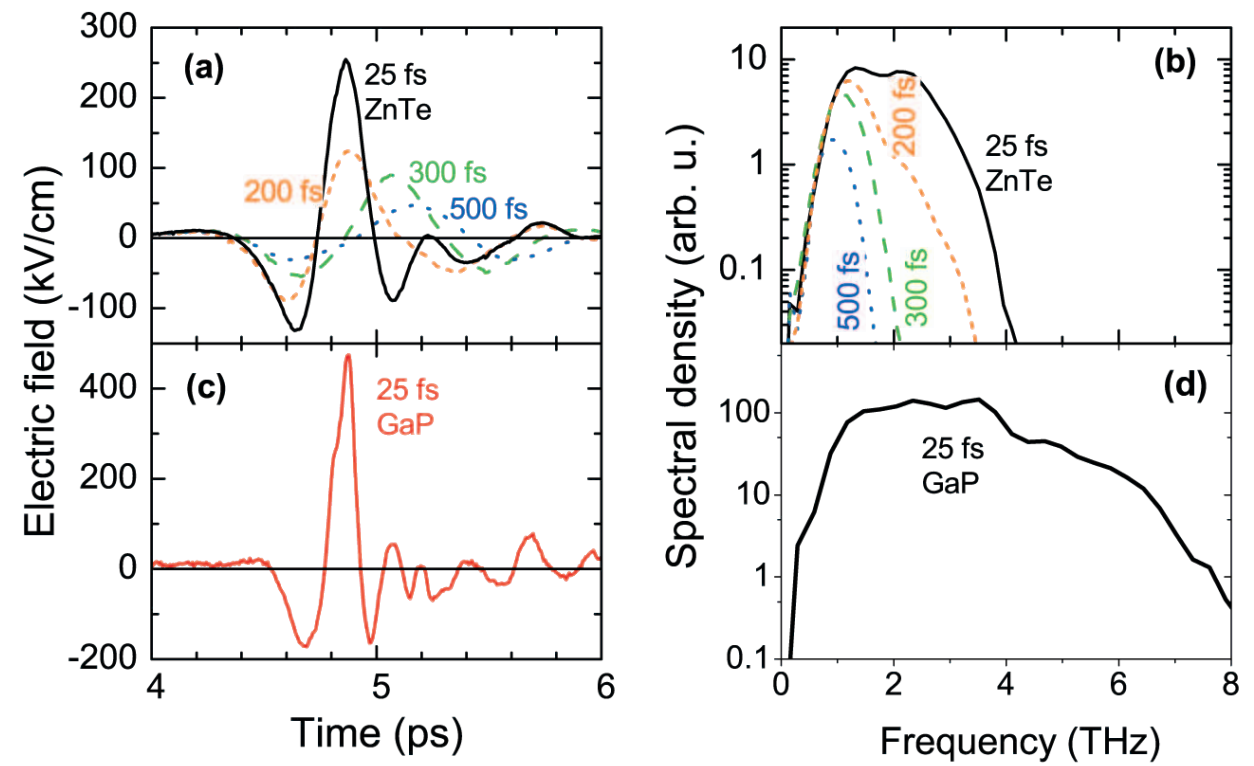

Figure 3.16: (a) THz pulses generated according to Figure 3.12 and measured by electro-optic sampling in a $0.4 \mathrm{~mm}$ thick ZnTe crystal. The pulse length of the optical pump pulse is indicated next to the transients. A shorter optical pulse yields higher $\mathrm{THz}$ field amplitudes. (b) Spectra of the $\mathrm{THz}$ transients measured in a ZnTe crystal. The bandwidth increases for shorter optical pump pulses. (c) $\mathrm{THz}$ pulse generated according to Figure 3.12 with an energy of $30 \mathrm{~nJ}$. The pulse was measured by electro-optic sampling in a $0.1 \mathrm{~mm}$ thick GaP crystal. (d) Spectrum of the $\mathrm{THz}$ transient measured in a GaP crystal. The larger bandwidth is clearly visible. 

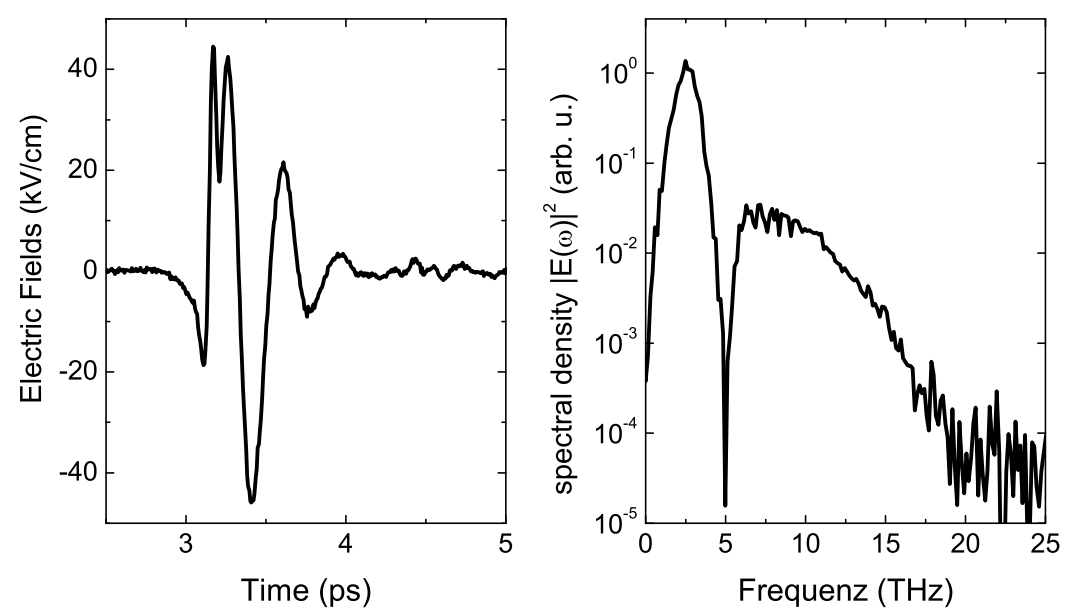

Figure 3.17: Ultrashort $\mathrm{THz}$ transient containing frequencies up to $20 \mathrm{THz}$. (a) Electric field vs. time. (b) Spectrum revealing the absorption band of the TO phonon of the ZnTe detection crystal.

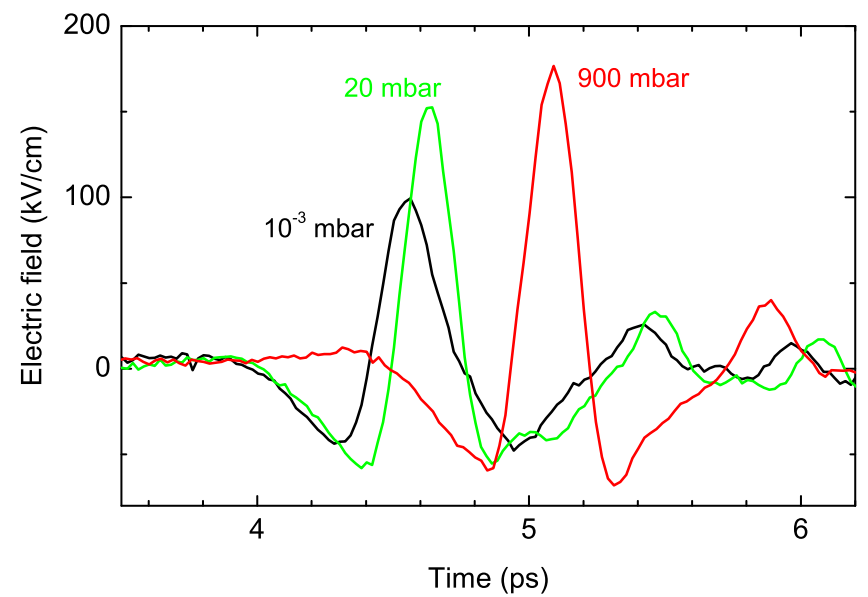

Figure 3.18: $\mathrm{THz}$ transients measured at varying pressure of the nitrogen gas. The transients recorded at low pressure, i.e $10^{-3}$ mbar, shows the contribution of the $\mathrm{BBO}$ crystal to the $\mathrm{THz}$ signal. 

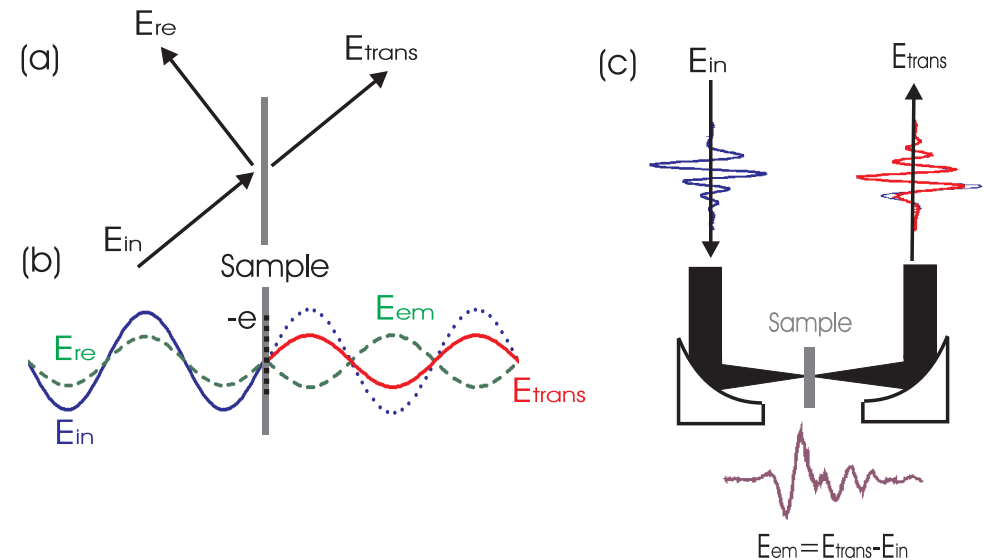

Figure 3.19: Transmission experiment on optically thin films: (a) Incident $\left(E_{\text {in }}\right)$, reflected $\left(E_{\mathrm{re}}\right)$ and transmitted $\left(E_{\text {trans }}\right)$ field. (b) Time dependent electric field $E_{\text {in }}$ (blue solid line) incident on an optically thin sample. The transmitted field $E_{\text {trans }}$ is composed of the superposition of the collectively emitted macroscopic field $E_{\mathrm{em}}$ (green solid line) and the incident field. In reflection, the emitted field $E_{\text {em }}$ is directly measured. (c) Experimental setup of the transmission experiment. The sample response is obtained by subtracting $E_{\text {trans }}-E_{\text {in }}=E_{\text {em }}$.

\section{4 $\mathrm{THz}$ transmission spectroscopy on thin films}

For thin films, the sample thickness $d$ is much shorter than the wavelength of the driving field $(d \ll \lambda)$. In this case one can treat the sample as a twodimensional layer. As a consequence, the reflectivity induced by the sample surface almost vanishes, because the reflection from the front and the rear facet interfere destructively. This is exact as long as the imaginary part of the refractive index is zero, i.e., as long as the incident radiation spectrum is off-resonant. In addition, it can be shown that the plasma frequency for electron motion parallel to the layer approaches zero for a two-dimensional sample [85].

As a result, the transversal driving electric field is constant over the sample thickness. Thus, charges in the sample will respond collectively to the driving field. The ensemble performs collective motions, which correspond to a macroscopic current density in the two-dimensional layer. Figure 3.19 (a) and (b) show such a situation: The incident field $E_{\text {in }}$ (blue solid line) leads to a collective acceleration of electrons in the sample. The coherent carrier motion, i.e., the macroscopic current density $j=\partial P / \partial t$, leads to the emission of a macroscopic electric field. The emitted field $E_{\mathrm{em}}$ is in the plain wave 
approximation:

$$
E_{\mathrm{em}}(t)=\frac{1}{2} j Z_{0} d,
$$

where $\mathrm{Z}_{0}$ is the vacuum impedance for the electric field and $d$ is the thickness of the sample. This emitted field acts back on the carriers in the twodimensional layer and modifies their motion. Hence, the field acting on the carriers, the so-called local field $\mathrm{E}_{\mathrm{loc}}$, is the superposition of the incident field and the emitted field [178]:

$$
E_{\mathrm{loc}}=E_{\mathrm{in}}+E_{\mathrm{em}} .
$$

In reflection geometry the emitted field can be measured directly, since the emitted field of a current density in a two dimensional layer is the same on both sides of the sample. Hence, $E_{\mathrm{re}}=E_{\mathrm{em}}$. In transmission geometry one measures the superposition of the incident field and the emitted field, i.e., $E_{\mathrm{in}}+E_{\mathrm{em}}=E_{\text {trans }}$, which is the field acting on the sample. Therefore, $E_{\mathrm{loc}}=E_{\text {trans }}$. In the thin film geometry depicted in Figure 3.19 one can easily extract the coherent response of the sample by extracting the emitted field with a simple subtraction:

$$
E_{\mathrm{em}}=E_{\text {trans }}-E_{\text {in }} .
$$

This is shown in Figure 3.19 (c).

The effect of strong radiative coupling is now demonstrated for a free carrier gas, e.g., in a doped semiconductor, where the frequency response follows the Drude theory. The emitted field is given by equation (3.19) and the current results from the local field at the sample: $j=E_{\mathrm{loc}} \sigma(\omega)$. In combination with equation (3.21) the transmission spectrum of the sample is

$$
T(\omega)=\left|\frac{E_{\text {trans }}(\omega)}{E_{\text {in }}(\omega)}\right|^{2}=\left|\frac{E_{\mathrm{loc}}}{E_{\mathrm{loc}}+1 / 2 E_{\mathrm{loc}} \sigma(\omega) Z_{0} d}\right|^{2}=\left|\frac{2}{2+\sigma(\omega) Z_{0} d}\right|^{2} .
$$

Hence, the frequency dependent transmission spectrum in the case of strong radiative coupling is completely different from the transmission spectrum obtained with Beer's law, which is valid in the case of negligible radiative coupling [178].

The main result from this paragraph for experimental applications is the deviation of the local field $\mathrm{E}_{\mathrm{loc}}$ acting on the sample from the incident field $E_{\text {in }}$. This deviation is expressed in equation (3.21), which allows to easily extract the local field at the sample [179]. 


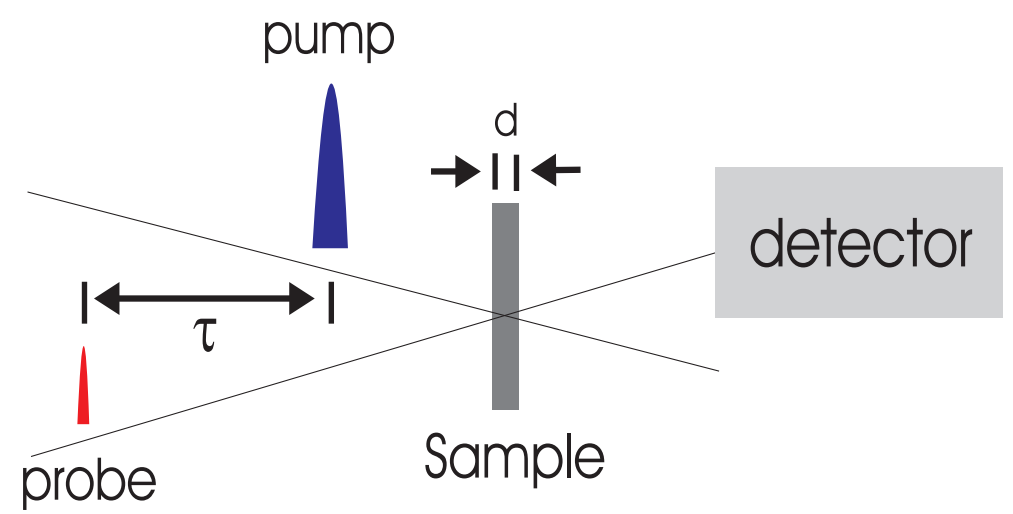

Figure 3.20: Setup for two-color pump-probe transmission spectroscopy: a strong pump pulse (blue) excites the sample. A weak probe pulse (red) interferes with the coherent polarization induced in the sample by the pump pulse. By variation of the time delay $\tau$ between the two pulses the dynamic absorption of the sample can be measured.

\subsection{Time-resolved two color pump-probe spec- troscopy}

A typical setup for time-resolved two-color pump-probe experiments is shown in Figure 3.20. An intense pump pulse induces a non-equilibrium situation in the sample. A subsequent weak probe pulse measures the transmission of the sample as a function of the time delay $\tau$. A time-resolved detection of the probe pulse, e.g., by electro-optic sampling, allows to measure the time-dependent transmission spectrum of the system under investigation. The time resolution is essentially determined by the length of the pump and probe pulses.

The pump-probe signal is a nonlinear signal which can be described by a third order nonlinear polarization $P_{\mathrm{NL}}$ [180]. The transmitted field can be calculated from the wave equation for the nonlinear polarization, which yields for an optically thin sample:

$$
E_{\text {probe }}(d, \tau, t)+E_{\mathrm{NL}}(\tau, t)=E_{\text {probe }}(0, \tau, t)+\frac{1}{2} d Z_{0} \frac{\partial P_{\mathrm{NL}^{(3)}}(\tau, t)}{\partial t} .
$$

The integrated pump probe signal is defined as the normalized difference between the probe intensity with and without the pump field [181]. It can be defined in the time and in the frequency domain.

$$
\frac{\Delta T(\tau, t)}{T_{0}}=\frac{\int_{-\infty}^{\infty} d t^{\prime} E_{\text {probe }}\left(\tau, t^{\prime}\right) E_{\mathrm{NL}}\left(\tau, t^{\prime}\right)}{\int_{-\infty}^{\infty} d t^{\prime}\left|E_{\text {probe }}\left(\tau, t^{\prime}\right)\right|^{2}}
$$




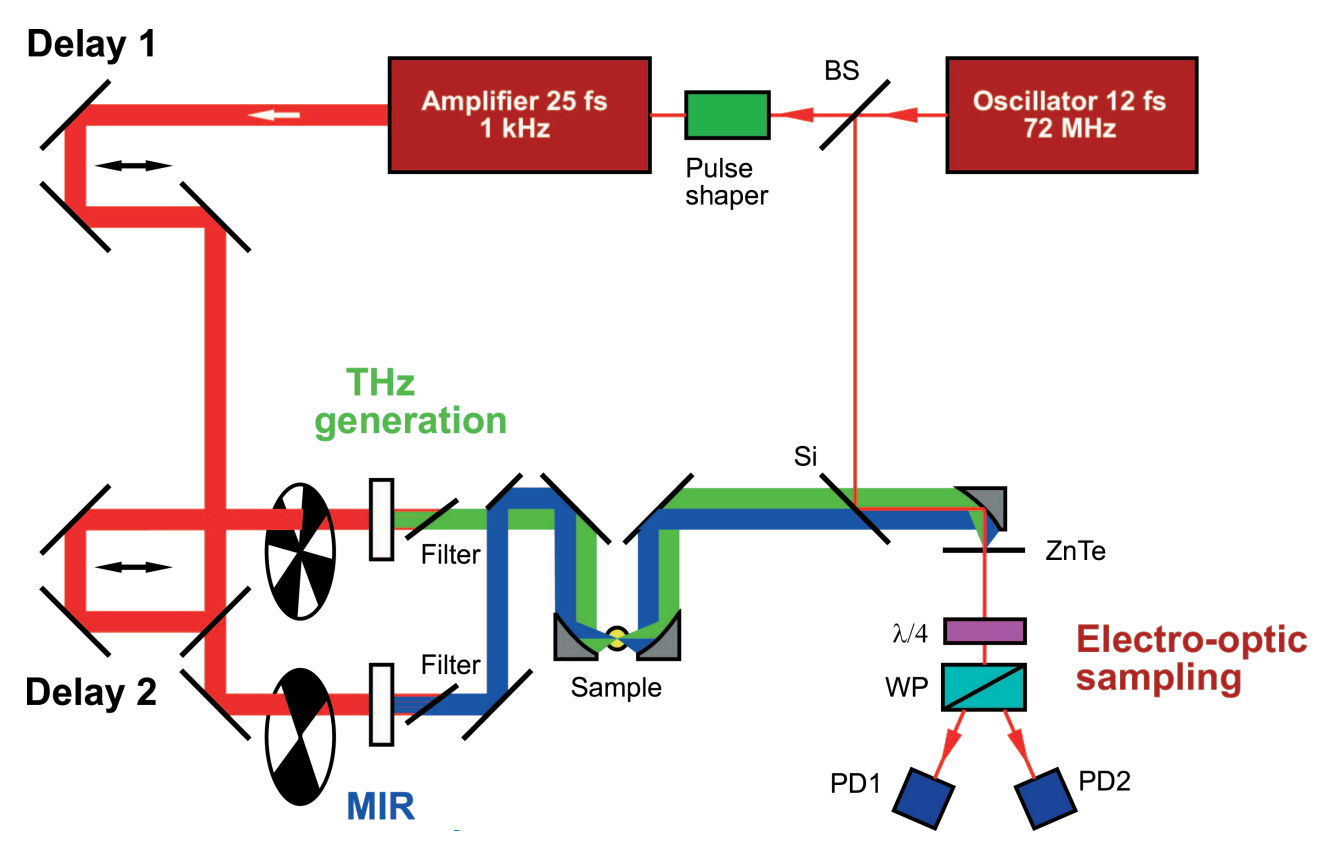

Figure 3.21: Setup for THz transmission and THz pump MIR probe experiments. $\mathrm{THz}$ and MIR radiation propagate parallel and are focused on the sample by a parabolic mirror. A second mirror collimates the beam which is then overlapped with the optical probe pulse and focused on an electro-optic crystal for detection via electro-optic sampling. Two individual choppers in the beam path of the $\mathrm{THz}$ and the MIR beam allow to block each beam independently.

$$
\frac{\Delta T(\tau, \omega)}{T_{0}}=\frac{\int_{-\infty}^{\infty} d \omega^{\prime} E_{\text {probe }}\left(\tau, \omega^{\prime}\right) E_{\mathrm{NL}}\left(\tau, \omega^{\prime}\right)}{\int_{-\infty}^{\infty} d \omega^{\prime}\left|E_{\text {probe }}\left(\tau, \omega^{\prime}\right)\right|^{2}}
$$

During the temporal overlap of pump and probe pulses, the pump pulse can interact both before and after the probe pulse with the sample and so-called coherent artifacts occur. These artifacts can also lead to signals for negative delay times $\tau \leq 0[180]$.

\subsubsection{THz-pump-MIR-probe setup}

The experimental setup used in this thesis is shown in Figure 3.21. The THz and the MIR beam propagate collinearly, so that they can be focused on the sample by a single parabolic mirror. The sample under investigation is placed in the focus of the mirror. The transmitted THz and MIR transients are collimated by a second parabolic mirror and overlapped with the optical probe pulse provided by the Ti:sapphire oscillator. A delay line (delay 2) introduces a relative temporal shift $\tau$ between the $\mathrm{THz}$ and the MIR pulse. Another delay line (delay 1) shifts the optical probe pulse for the electro-optic detection 
with respect to the MIR and $\mathrm{THz}$ pulses. This corresponds to the sampling time coordinate $t$. The $\mathrm{THz} / \mathrm{MIR}$ radiation and the optical probe pulse are overlapped using a $0.5 \mathrm{~mm}$ thick undoped silicon plate, which reflects the $800 \mathrm{~nm}$ probe pulse, but is transparent for the long wavelength radiation. In this configuration, both beams, which can be chopped individually, can be used as pump and probe pulses. For the $\mathrm{THz}$ transmission measurements the MIR beam is blocked. For the two-color pump-probe measurements, the $\mathrm{THz}$ beam was chopped with a frequency of $500 \mathrm{~Hz}$ and the MIR beam was chopped with a frequency of $125 \mathrm{~Hz}$. This allows to measure the following configurations:

- $E_{\mathrm{both}}:=1 \times E_{\mathrm{THz}}+1 \times E_{\mathrm{MIR}}$, i.e., both the MIR and the THz pulse are incident on the sample.

- $E_{\mathrm{THz}}:=1 \times E_{\mathrm{THz}}+0 \times E_{\mathrm{MIR}}$, i.e., only the $\mathrm{THz}$ pulse can propagate, the MIR pulse is blocked.

- $E_{\mathrm{MIR}}:=0 \times E_{\mathrm{THz}}+1 \times E_{\mathrm{MIR}}$, i.e., only the MIR pulse can propagate, the $\mathrm{THz}$ pulse is blocked.

- $E_{b g}:=0 \times E_{\mathrm{THz}}+0 \times E_{\mathrm{MIR}}$, both pulses are blocked.

By subtracting $E_{\mathrm{both}}-E_{\mathrm{THz}}-E_{\mathrm{MIR}}$ one can extract the nonlinear response of the sample. In this thesis the $\mathrm{THz}$ beam was used as a pump and the MIR as probe. 


\section{Chapter 4}

\section{High field $\mathrm{THz}$ response of n-type gallium arsenide at room temperature}

As a first application of the experimental and theoretical concepts discussed in the previous chapters, a $\mathrm{THz}$ transmission experiment on a thin layer of n-doped GaAs is presented. Excitation with ultrashort, intense $\mathrm{THz}$ pulses of the sample yields a nonlinear oscillatory response which originates from radiative transitions of electrons in the potentials of the donor atoms. The signal vanishes for very low pump intensities where the sample response is Drude like. Data of linear and nonlinear measurements on samples with different doping densities are shown. For the interpretation of the experimental data, a quantum mechanical discrete state (QMDS) model is developed and model calculations are presented.

\subsection{Sample geometry}

The sample consists of a $500 \mathrm{~nm}$ thin silicon doped n-type gallium arsenide layer clad between two $300 \mathrm{~nm}$ thick $\mathrm{Al}_{0.4} \mathrm{Ga}_{0.6}$ As layers. The layers were grown on a $500 \mu \mathrm{m}$ thick semi-insulating gallium arsenide substrate. Two samples with different doping concentrations $\mathrm{N}_{d}$ were investigated:

- HD: $N_{d}=9 \times 10^{16} \mathrm{~cm}^{-3}$,

- LD: $N_{d}=2 \times 10^{16} \mathrm{~cm}^{-3}$.

A sketch of the sample is shown in Figure 4.1 (a). The substrate layer provides the necessary mechanical stability but influences the measurement otherwise by introducing dispersion or spurious nonlinearities from unintended 
(a)

(b)

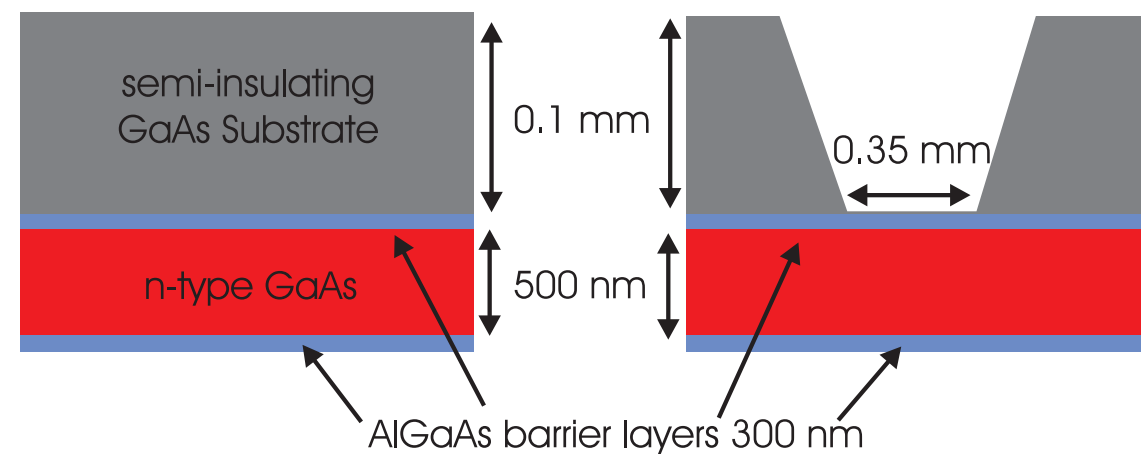

Figure 4.1: n-type gallium arsenide sample (a) as grown; (b) the substrate is locally removed by wet chemical etching. The dimensions (not to scale) are given in the graph.

doping. Therefore it was removed locally for the nonlinear measurement by selective wet chemical etching [182]. The etching solution utilized hydrogen peroxide, which leads to an oxidation of the substrate and ammonium hydroxide which removes the oxidized layer [183]. The etching velocity in the gallium arsenide substrate is ten times higher than in the $\mathrm{Al}_{0.4} \mathrm{Ga}_{0.6}$ As layers, which function as etch stops. Figure 4.2 (a) and (b) show the etched regions of samples HD and LD, respectively.

A schematic of the etching process is shown in Figure 4.2 (c). The diameter of the region where the substrate is removed must be larger than the diameter of the $\mathrm{THz}$ pump beam in the focus. Otherwise the etched spot could result in a spatial filtering of the $\mathrm{THz}$ wave because the focal spot size depends on the $\mathrm{THz}$ wavelength. For both samples the diameter is $\geq 350 \mu \mathrm{m}$ compared to a focus diameter of $\leq 200 \mu \mathrm{m}$. For mechanical stability the approximately $100 \mu \mathrm{m}$ thin sample was attached to a $400 \mu \mathrm{m}$ thick diamond plate. Diamond is an ideal substrate since it is transparent in the $\mathrm{THz}$ and MIR range and has a high thermal conductivity. In addition, it adds only negligible dispersion in that wavelength range [184].

\subsection{Experimental Results}

\section{Linear $\mathrm{THz}$ transmission measurements}

A schematic of the linear measurement is shown in the inset of Figure 4.3. In this experiment, the sample was investigated as grown. In order to extract the signal from the doped layer, the measured transmitted pulses were 

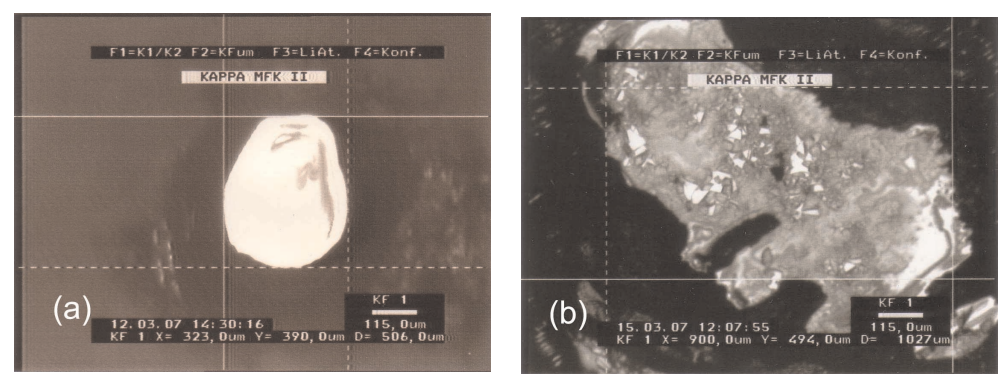

(c)

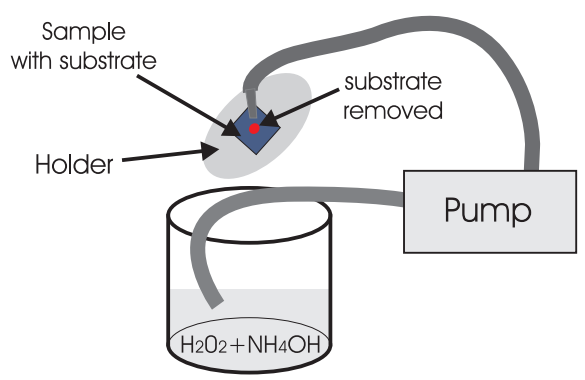

Figure 4.2: picture of sample HD (a) and sample LD (b) taken under a microscope. (c) Etching with a $\mathrm{H}_{2} \mathrm{O}_{2} / \mathrm{NH}_{4} \mathrm{OH}$-solution following [182]

corrected for the reflection losses and the dispersion added by the substrate. Figure 4.3 (a) shows the measured transients of the incident $E_{\text {in }}(t)$ (black dashed line) and transmitted $E_{\text {trans }}(t)$ (blue dotted line) electric field after correction for dispersion introduced by the gallium arsenide substrate. The transmitted pulse displays a reduced amplitude mainly due to reflection losses at the sample facets, and a slightly negative phase shift. The solid line in Figure 4.3 (a) is a Drude fit to the transmitted pulse assuming a scattering time of $\tau_{\text {Drude }}=150 \mathrm{fs}$. It shows excellent agreement with the measured transient. The Fourier transforms of the pulses are shown in Figure 4.3 (b). The difference spectrum $\left|E_{\text {trans }}(\nu)\right|^{2}-\left|E_{\text {in }}(\nu)\right|^{2}$ (red diamonds) is slightly redshifted relative to that of the driving pulse without any spectrally narrow features.

\section{Nonlinear propagation experiment}

The nonlinear measurement is presented in Figure 4.4. For this measurement the substrate was removed as shown in Figure 4.1 (b) and the sample was placed in the focus of the $\mathrm{THz}$ beam between the parabolic mirrors (inset Figure 4.4 (b)). Due to the thickness of the sample, which is much smaller than the $\mathrm{THz}$ wavelength, the reflection loss at the sample is zero. Figure 4.4 (a) shows the driving $\mathrm{THz}$ field $E_{\text {in }}(t)$ with a maximum amplitude of approximately $45 \mathrm{kV} / \mathrm{cm}$ (dashed line) and the transmitted pulse $E_{\text {trans }}(t)$ (blue solid 

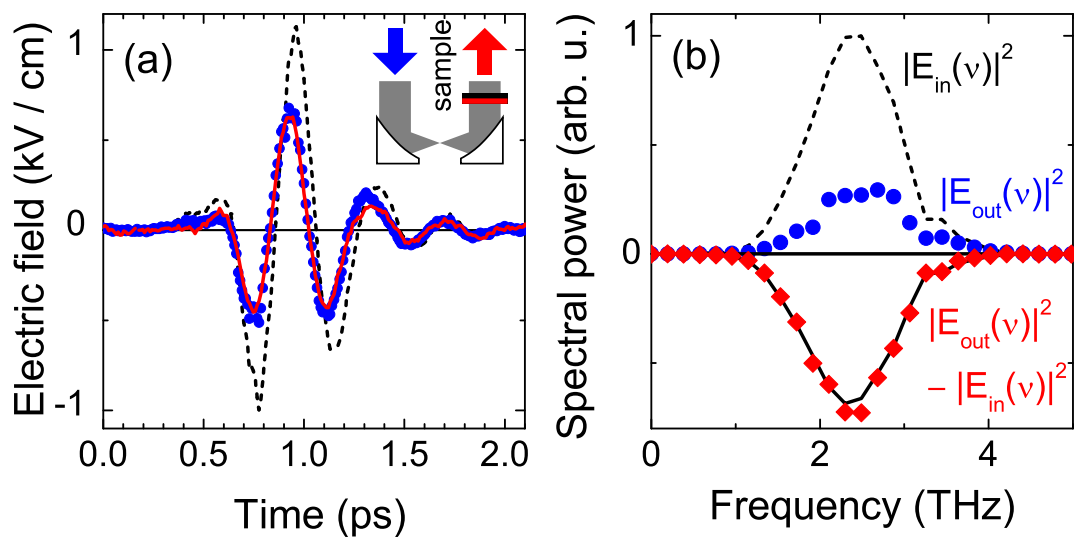

Figure 4.3: Linear measurement of sample HD: (a) Linear propagation of $\mathrm{THz}$ pulses through an n-type GaAs layer. The electric field of the incident pulse $E_{\text {in }}(t)$ (black dashed line) and the transmitted pulse $E_{\text {trans }}(t)$ (blue dotted line) are plotted as a function of time. The expected $E_{\text {trans }}(t)$ according to the Drude model is shown for comparison (red solid line). Inset: experimental setup with the sample placed in the parallel $\mathrm{THz}$ beam. (b) Corresponding power spectra: $\left|E_{\text {trans }}(\nu)\right|^{2}-\left|E_{\text {in }}(\nu)\right|^{2}$ (red diamonds) and the expected Drude result (black solid line)

line). The corresponding power spectra are depicted in Figure 4.4 (b). The difference signal $E_{\text {trans }}(t)-E_{\text {in }}(t)$, which corresponds to the emitted field of the n-type GaAs layer, is shown in Figure 4.4 (c). It reveals a pronounced oscillation at times $\mathrm{t}>0.5 \mathrm{ps}$, which is already noticeable in the transmitted pulse in Figure 4.4 (b).

The spectral difference signal $\left|E_{\text {trans }}(\nu)\right|^{2}-\left|E_{\text {in }}(\nu)\right|^{2}$ is shown in Figure 4.4 (d) (red diamonds). The most prominent feature is the spectrally narrow positive peak at $2 \mathrm{THz}$ revealing a coherent emission at that frequency. On both sides of the emission peak, one observes broadband $\mathrm{THz}$ absorption. These features differ strongly from the expected Drude result (black solid line) which perfectly match the sample response in the linear measurement.

In Figure 4.5 (a), the difference signal $E_{\text {trans }}(t)-E_{\text {in }}(t)$ is plotted for various amplitudes $E_{\max }$ of the incident $\mathrm{THz}$ pulse. The amplitudes are indicated in the graph above the transients. The discussion concentrates on the oscillatory signal after the exciting pulse (shown in colored solid lines). The Fourier transforms of these signals are shown in Figure 4.5 (b). For 


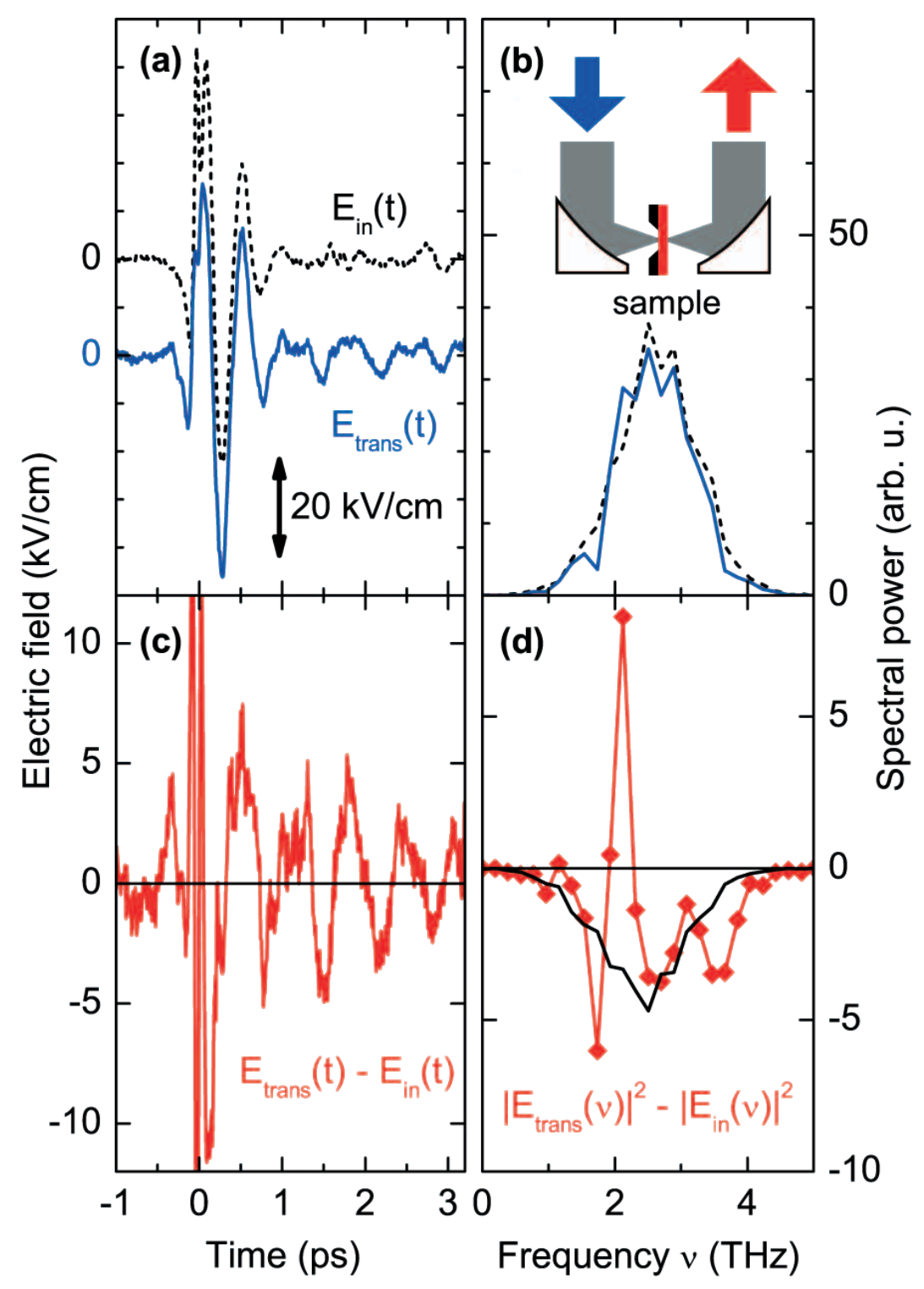

Figure 4.4: Nonlinear propagation of the THz pulses through the n-type GaAs layer (sample HD) without the substrate. (a) incident pulse $E_{\text {in }}(t)$ (black dashed line), transmitted pulse $E_{\text {trans }}$ (blue solid line) (b) Corresponding power spectra. Inset: experimental setup. (c) The difference signal $E_{\text {trans }}(t)-E_{\text {in }}(t)$ shows weakly damped oscillations at a frequency of $\nu=2 \mathrm{THz}$. (d) The difference spectra (red diamonds) exhibits a pronounced, positive peak at $2 \mathrm{THz}$, which is in distinct contrast to the expected Drude response (solid). 

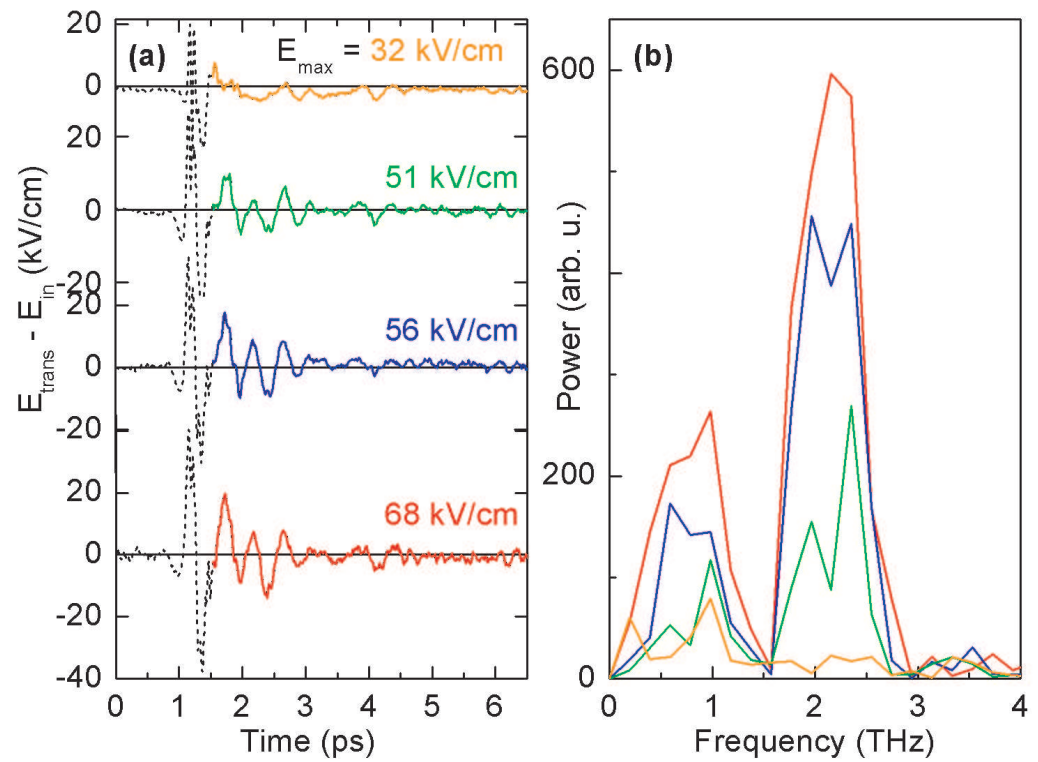

Figure 4.5: (a) Difference signals $E_{\text {out }}(t)-E_{\text {in }}(t)$ for several amplitudes $E_{\max }$ of the incident $\mathrm{THz}$ pulse (amplitude indicated above the transients). The coherent oscillations after the main pulse (colored solid lines) occur above a threshold of approximately $40 \mathrm{kV} / \mathrm{cm}$. (b) Fourier transforms of $E_{\text {out }}(t)-E_{\text {in }}(t)$ after the main pulse.

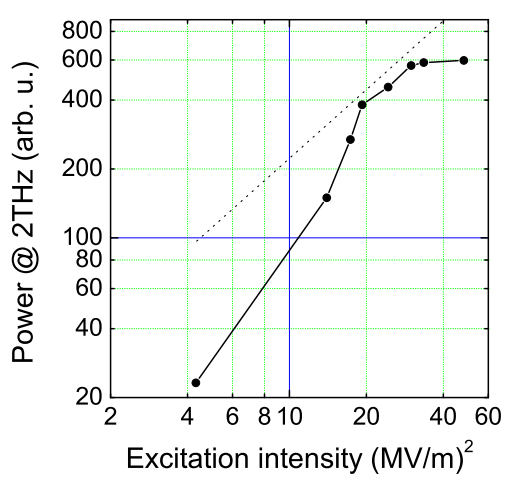

Figure 4.6: Spectral power at $2 \mathrm{THz}$ as a function of the intensity of the driving field $E_{\max }$ (black solid line) on a double logarithmic scale. A linear dependence is shown (black dashed line) for comparison. 

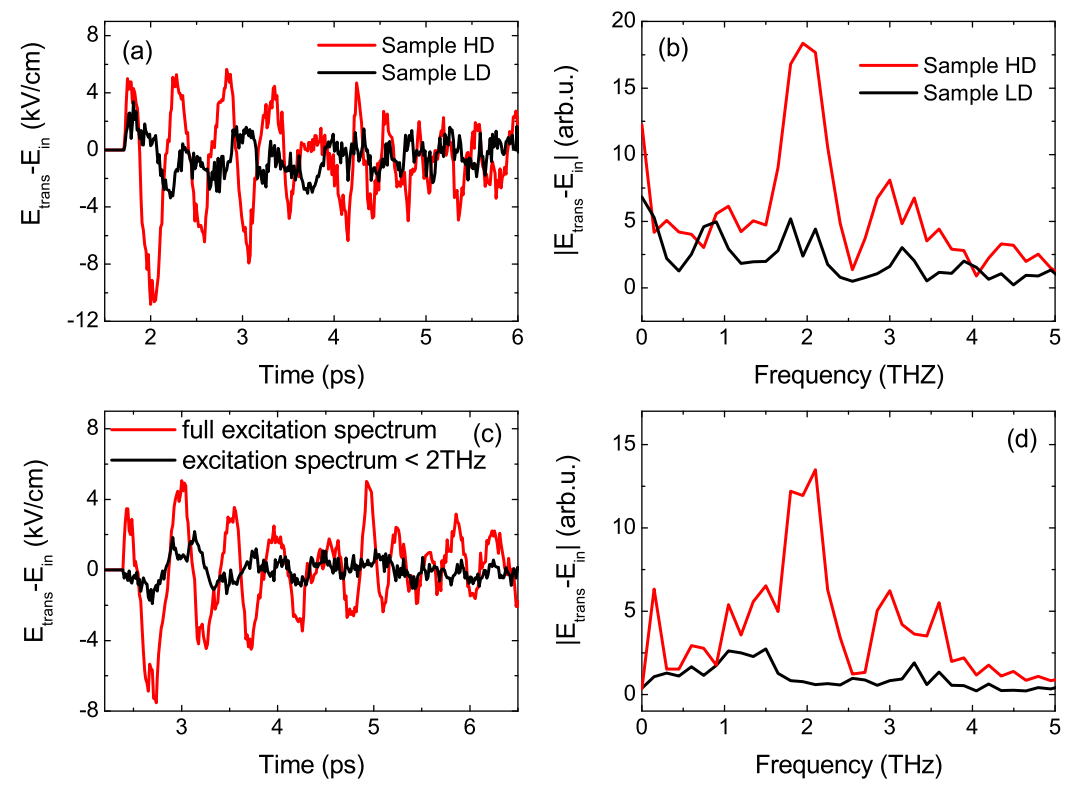

Figure 4.7: Comparison of the difference signal $E_{\text {trans }}-E_{\text {in }}$ measured in sample LD (black solid line) with the signal measured in sample HD (red solid line) for comparable intensities of the incident excitation pulses $\left(\mathrm{E}_{\mathrm{in}}^{\mathrm{HD}} \approx 70 \mathrm{kV} / \mathrm{cm}\right.$ compared to $\mathrm{E}_{\text {in }}^{\mathrm{LD}} \approx 100 \mathrm{kV} / \mathrm{cm}$ ). (a) The oscillatory features after the excitation pulse (not shown) occur only in sample HD. (b) The characteristic peak at $2 \mathrm{THz}$ occurs for the measurement in sample HD. The spectrum measured in sample LD is flat. (c) Difference signal measured in sample HD with a broadband $\mathrm{THz}$ pulse (red solid line) and a $\mathrm{THz}$ pulse which contains only frequencies below $2 \mathrm{THz}$ (black solid line). In the latter case no oscillations after the pulse are observed. (d) Spectra of the difference signals shown in (c).

the lowest driving field of $E_{\max }=32 \mathrm{kV} / \mathrm{cm}$ the oscillations are absent. By increasing the driving field to $E_{\max } \geq 50 \mathrm{kV} / \mathrm{cm}$, clear oscillations are found which give rise to the $2 \mathrm{THz}$ peak in the spectrum. Interestingly, the coherent emission lasts for more than a picosecond, which is substantially longer than the Drude scattering time of 150 fs.

The power dependence of the spectral feature at $2 \mathrm{THz}$ has been analyzed in Figure 4.6. The signal (black solid line) increases superlinearly with the intensity of the driving field and saturates at very high fields. A linear dependence is shown for comparison (black dashed line).

The nonlinear measurement was repeated on sample LD. The result are shown in Figure 4.7 (a) and (b). Even though the excitation intensity is 
(a)

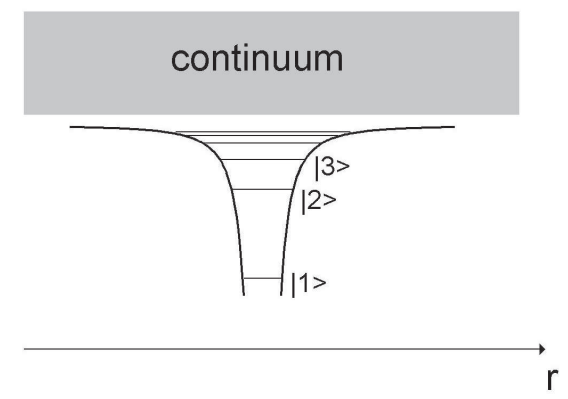

(b) $\quad-e \cdot E \cdot r$

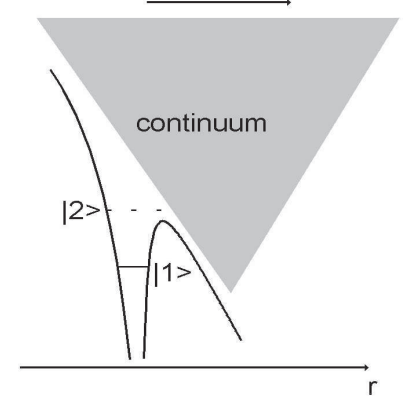

Figure 4.8: (a) Shallow donor potential in thermal equilibrium and (b) under the influence of a strong electric field.

$100 \mathrm{kV} / \mathrm{cm}$, no oscillations after the main pulse are observed. In consequence the spectrum in Figure 4.7 (b) does not show any distinct structures. In addition, the oscillatory signal is not observed after excitation of sample HD with a spectrally shaped $\mathrm{THz}$ pulse which contains only frequencies below $2 \mathrm{THz}$. This is shown in Figure 4.7 (c) and (d).

\subsection{Discussion}

In the following, the data presented in the previous section is analyzed and the physical mechanism underlying the observed coherent emission is discussed. The chapter concludes by presenting model calculations based on the solution of the one-electron Schrödinger equation in a disordered potential landscape.

\subsubsection{Qualitative discussion}

The following paragraph shows that the coherent nonlinear signal is generated in a stimulated emission process from free carrier states into impurity ground states. Already the observation that the signal vanishes when the doping concentration of the otherwise identical sample is reduced or if the generated frequency is not contained in the excitation pulse strongly suggest that inter impurity transitions are relevant for the experimentally observed phenomena. The basic idea is explained in Figure 4.8 (a) and (b) showing the donor potential in real space without and with a strong electric field, respectively. In the case where no electric field is present on the sample, the donor potential leads to discrete localized electronic states which lie energetically below the conduction band edge. An incident $\mathrm{THz}$ pulse can promote 
an electron in the impurity ground state to continuum states via the optical dipole allowed transition. Due to the strong localization of the ground state wavefunction compared to the wavefunction of the continuum states, the transition dipole moment is rather small. On the other hand, the potential gradient provided by the strong electric field of the incident $\mathrm{THz}$ pulse adds to the impurity potential and leads to a significantly reduced binding, or even breaking, of the localized states, as shown in Figure 4.8 (b). Thus trapped carriers in the impurity ground state are freed by above threshold or tunneling ionization, respectively. The ionization field strength can be estimated using the equivalent Bohr radius to $E_{\text {ion }}=E_{1 S}\left(e \cdot a_{B}\right)^{-1} \approx 6 \mathrm{kV} / \mathrm{cm}$ for $a_{B}=10 \mathrm{~nm}$ and a binding energy $E_{1 S}=6 \mathrm{meV}$. Therefore the driving pulse is strong enough to ionize the initially neutral impurities and the fieldionization process dominates the optical transition to continuum states.

In sample HD about $30 \%$ of the donor electrons are trapped in the donor ground state at room temperature, compared to only approximately $10 \%$ for sample LD (see Figure 2.5). In absolute numbers, 15 times more impurities can be ionized in sample HD compared to sample LD. The dynamics of the THz field induced effect is shown in Figure 4.9. The initially neutral impurities are ionized by the electric field of the incident $\mathrm{THz}$ pulse. In consequence, electrons from the impurity ground state are transferred to delocalized continuum states, thus creating a non-thermal carrier distribution. The ensemble of carriers in the conduction band contains the initially free carriers and the carriers which result from ionization by the electric field of the THz pulse. Subsequent thermalization through electron-electron scattering leads to the occupation of excited impurity states and energetically low conduction band states. In impurities with a populated excited state and an unpopulated ground state, stimulated emission can occur on the corresponding dipole-allowed transition, which overlaps with the spectrum of the $\mathrm{THz}$ pump pulse. As a result, the $\mathrm{THz}$ pulse is first amplified and eventually saturates the optical gain, leading to the emission of a phase-coherent superradiant wave. This also explains the observed dependance of the stimulated emission signal on the maximum incident field strength $E_{\max }$ shown in Figure 4.6. For low $E_{\max }$ the field strength is too low for efficient ionization. For high $E_{\max }$ the coherent emission saturates, since at most all electrons can emit coherently.

The derived mechanism explains the observed effect also quantitatively. Still an open question is in particular the observed emission frequency of approximately $2 \mathrm{THz}$, which does not match the transition frequency of isolated impurities of $1.1 \mathrm{THz}$ [37]. 
(a)

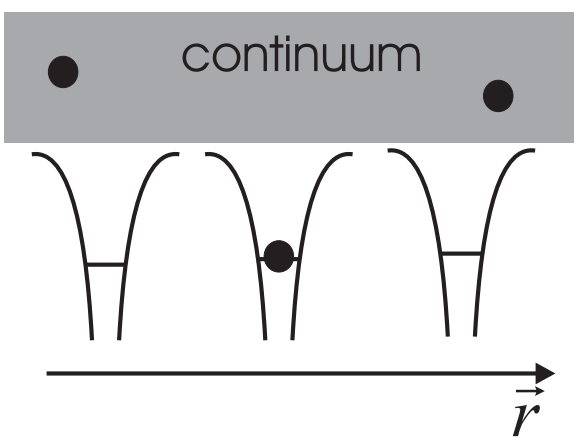

(c)

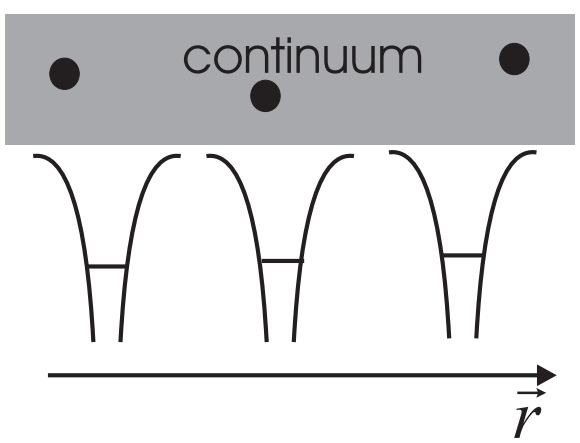

(b)

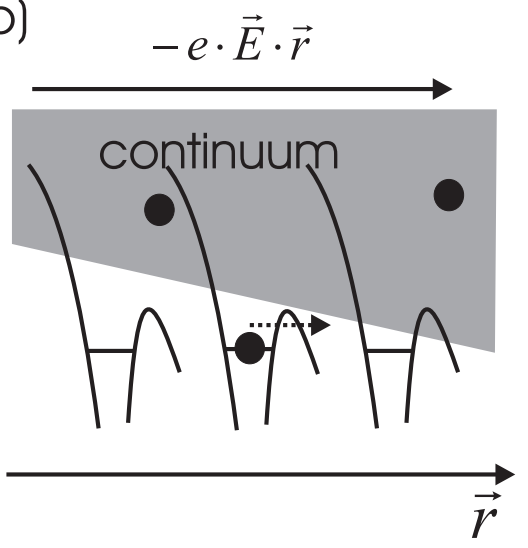

(d)

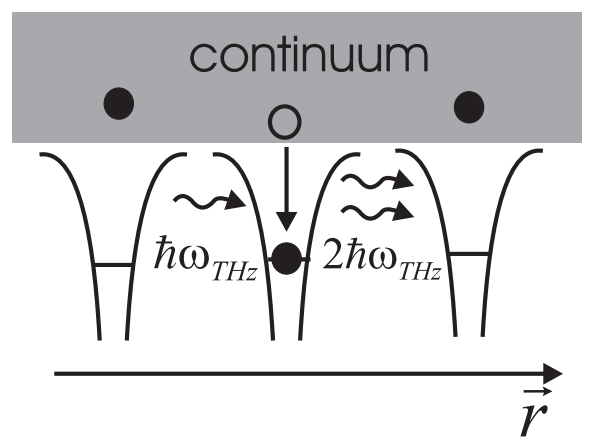

Figure 4.9: THz field induced coherent emission: (a) equilibrium situation before the excitation. A fraction of the donor potentials is neutral. (b) The electric field of the incident $\mathrm{THz}$ pulse leads to ionization of the previously neutral impurities by promoting carriers to continuum states in the conduction band. (c) The carriers thermalize by electron-electron scattering on a sub-picosecond time scale, thus populating higher lying impurity and continuum states. (d) In impurities with an occupied P-like excited state and an unpopulated S-like ground state, stimulated emission can occur on the corresponding dipole-allowed transition. The emission is seeded by the $\mathrm{THz}$ pump pulse which spectrally overlaps with the transition frequency. 


\subsubsection{Quantum mechanical discrete state model (QMDS)}

In the following paragraph, a simple model provides a better understanding of the electron wavefunction in the potential landscape which results from the introduction of random impurity potentials. The medium is represented by a rectangular box with a side length of $67 \mathrm{~nm}$. Inside the box 30 Coulomb potentials screened by the GaAs dielectric constant were randomly distributed and represent the impurity potentials. The amount of $\mathrm{q}=30$ impurities distributed in the volume $(67 \mathrm{~nm})^{3}$ corresponds to the doping concentration of sample HD. The average distance between two impurities is approximately $20 \mathrm{~nm}$ and therefore roughly twice the donor Bohr radius. The random distribution of the impurities, however, allows for a variation of the inter donor distance. In a donor which is spatially separated by less than the average distance from other donors, the electron wavefunction of the donor ground state would delocalize over two or more impurities. If for example two donors were by chance located very closely together, this would result in a heliumlike impurity potential with a higher ionization energy and a lower dipole moment compared to an isolated impurity. This analogy already shows that the random distribution of the donor atoms during the growth of the crystal has an influence on the $\mathrm{THz}$ field response. The formation of impurity bands was already discussed in Section 2.1.3.

After distributing the impurity potentials, the one-electron Schrödinger equation is solved in the medium with the finite difference method. In the calculations each dimension was discretized in 7 steps, corresponding to a spatial grid of approximately $9.5 \mathrm{~nm}$, which is in the order of the Bohr radius. This value was found as the best trade-off between spatial resolution and calculation expense, which increases to the sixth power with the number of grid points. An increase to more grid points leads to a significant increase in calculation time without a significant modification of the result.

An advantage of the QMDS model is that it accounts for the effects of disorder in the impurity distribution. Under the conditions defined above, which correspond to sample HD, roughly $80 \%$ of the donors have a localized ground state wavefunction. The excited states are much less localized and all energetically higher states show complete delocalization.

In order to test the QMDS model, the transmission spectrum of an isolated impurity $(\mathrm{q}=1)$ is studied. The joint density of $1 \mathrm{~S} \leftrightarrow \mathrm{nP}$ transition (DOT) corresponds to the absorption spectrum of ground state to excited state transitions in the medium for the case that all impurity ground states are occupied. Hereby states which show the highest probability density at the impurity are considered ground states. Note that in this definition the energetically lowest states are not necessarily impurity ground states. The 

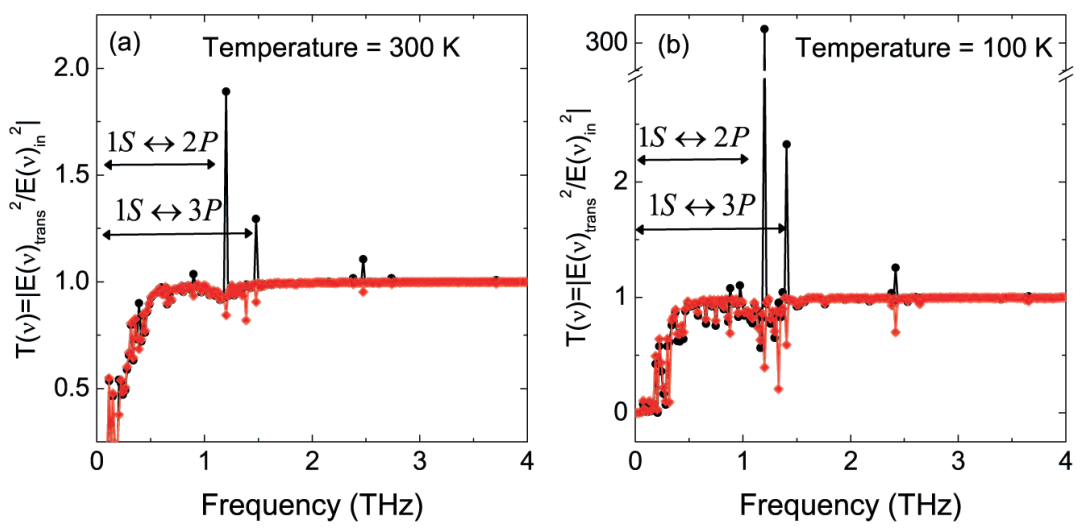

Figure 4.10: Transmission spectrum of an isolated impurity $(\mathrm{q}=1)$ calculated in the QMDS model. (a) Results for a temperature of $300 \mathrm{~K}$ of the electron distribution. Transmission in an inverted system (black dotted line), i.e. the impurity ground state is ionized and in thermal equilibrium (red dotted line). (b) Transmission at a temperature $\mathrm{T}=100 \mathrm{~K}$ for an inverted system (black dotted line) and in thermal equilibrium (red dotted line).

joint density of $1 \mathrm{~S} \leftrightarrow \mathrm{nP}$ transitions (DOT) reads:

$$
Z(\omega)=\omega \sum_{i=1}^{q} \sum_{k}\left|\left\langle k|\vec{r}| 1 S_{i}\right\rangle\right|^{2} \delta\left(E_{k}-E_{|1 S\rangle}-\hbar \omega\right)
$$

The first summation includes all ground state wavefunctions, the second is a summation over all Eigenstates of the system. The ground state $|1 S\rangle_{i}$ of impurity $i$ has been identified as the state with the maximum probability density at the impurity $i$. $1 \mathrm{~S} \leftrightarrow \mathrm{nP}$ transitions denote transitions between the identified impurity ground state $|1 S\rangle_{i}$ and the Eigenstates $|k\rangle$. The transmission spectrum is obtained by occupying the various states calculated in the QMDS model with an electron distribution. For the linear transmission spectrum, a Boltzmann distribution is used, because at the given low doping concentrations the carrier gas is non-degenerate. The nonlinear transmission spectrum is obtained by removing electrons from the energetically lowest states, thus creating a population inversion. The removed carriers are added to the carrier distribution at higher energies.

The results of the QMDS model for an isolated impurity are shown in Figure 4.10 (a) and (b). The characteristic temperature of the free, i.e., conduction band, electron gas has been set to $\mathrm{T}=300 \mathrm{~K}$ (Figure 4.10 (a)) and 
(a)

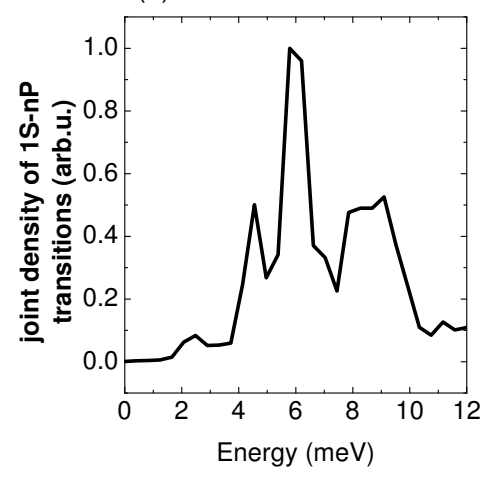

(b)

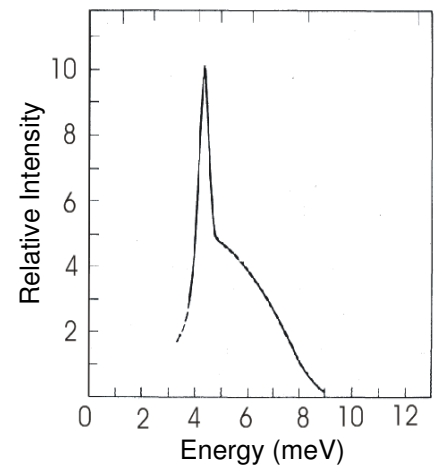

Figure 4.11: (a) Joint density of $1 \mathrm{~S} \leftrightarrow \mathrm{nP}$ transitions $\mathrm{q}=30$ impurities in a rectangular box with sidelength of $67 \mathrm{~nm}$. (b) photoluminescence spectrum of n-doped GaAs with a doping concentration of $\mathrm{N}_{d}=2.5 \times 10^{14} \mathrm{~cm}^{-3}$ after reference [37]

$\mathrm{T}=100 \mathrm{~K}$ (Figure 4.10 (b)). It should be stressed that the case $\mathrm{q}=1$ corresponds to a doping concentration of $\mathrm{N}_{d}=3.3 \times 10^{15} \mathrm{~cm}^{-3}$. The transmission spectrum of the isolated impurity (black dotted line) shows distinct peaks at $1.2 \mathrm{THz}$ and $1.3 \mathrm{THz}$. These peaks correspond to the $1 \mathrm{~S} \rightarrow 2 \mathrm{P}$ and to the $1 \mathrm{~S} \rightarrow 3 \mathrm{P}$ transition, respectively. By decreasing the temperature, the amplitude of the peaks increases drastically, which results from a higher occupation difference between the ground state and excited states. At room temperature, i.e., $\mathrm{T}=300 \mathrm{~K}$, the non-inverted isolated impurity shows a Drude like transmission spectrum. By decreasing the temperature to $\mathrm{T}=100 \mathrm{~K}$ the impurity transitions appear as minima in the transmission spectrum of the noninverted impurity. The inverse effect, i.e., radiative recombination, leads to the photoluminecense spectrum shown in Figure 2.8 and Figure 4.11 (b). At cold temperatures, the system can therefore not be described with a simple Drude response.

The results shown in Figure 4.10 show that the QMDS model accurately describes the transmission spectrum of an isolated impurity. Therefore the model is applied to systems which correspond to sample LD and HD.

At higher doping densities ( $q>1$ ), delocalization of the electronic wavefunction over several impurities influences the transmission spectrum of the system. This effect is discussed in the following. The joint density of $1 \mathrm{~S} \leftrightarrow \mathrm{nP}$ transitions calculated in a system which corresponds to sample HD is shown in Figure 4.11 (a). In Figure 4.11 (b) the photoluminescence spectrum of an 
isolated impurity measured by Melngailis et al. [37] is shown for comparison. Interestingly in the DOT-spectrum the maximum is blue shifted compared to the isolated impurity. The result shown in Figure 4.11 is interpreted as follows:

- The peak in the joint DOT slightly above $4 \mathrm{meV}$, which corresponds to the energy of the photoluminescence maximum in Figure 4.11 (a), originates from isolated impurities in the disordered potential, i.e., from donor atoms with a higher distance to their neighbors than the average distance of $20 \mathrm{~nm}$. At this doping concentration in the calculation, the sub-ensemble of isolated-like impurities is rather small. Therefore, the amplitude of the corresponding peak in the DOT spectrum is rather low.

- The global maximum of the DOT-spectrum at $6 \mathrm{meV}$ corresponds to $1 \mathrm{~S} \leftrightarrow \mathrm{nP}$ transitions where the ground state energy is lowered due to a delocalization over more than one impurity. In addition, the excited state wavefunction still shows a significant degree of localization, which results in the strongest oscillator strength in the ensemble at an energy of $6 \mathrm{meV}$.

- The shoulder in the DOT-spectrum at higher energies corresponds to transitions between ground states and higher excited impurity or continuum states. This shoulder is also present in the photoluminescence spectrum in Figure 4.11.

A transmission spectrum calculated with the QMDS model is shown in Figure 4.12. For the linear response (black dots) a thermal Boltzmann distribution of the carriers at room temperature is assumed. For comparison, the absorption spectrum calculated with the Drude model is plotted (blue solid line), assuming a Drude scattering time of $\tau=150 \mathrm{fs}$. Both curves show excellent agreement. To model the nonlinear response, a population inversion is created by ionizing $\mathrm{q} / 2$ of the energetically lowest states. This corresponds to a population inversion of $50 \%$ and simulates the situation after the sample was excited with a strong $\mathrm{THz}$ pulse, which ionized initially neutral impurities. Note that the sample temperature is still approximately 300 K, i.e., room temperature. The transmission spectrum for the non-equilibrium situation (red diamonds), shows a strong maximum at $2 \mathrm{THz}$, exactly the same frequency found in the experiment.

It must be stressed that the spectral position, height and width of the peak in the transmission spectrum result from the random distribution of donor atoms in the material. As the calculation of the QMDS model includes 


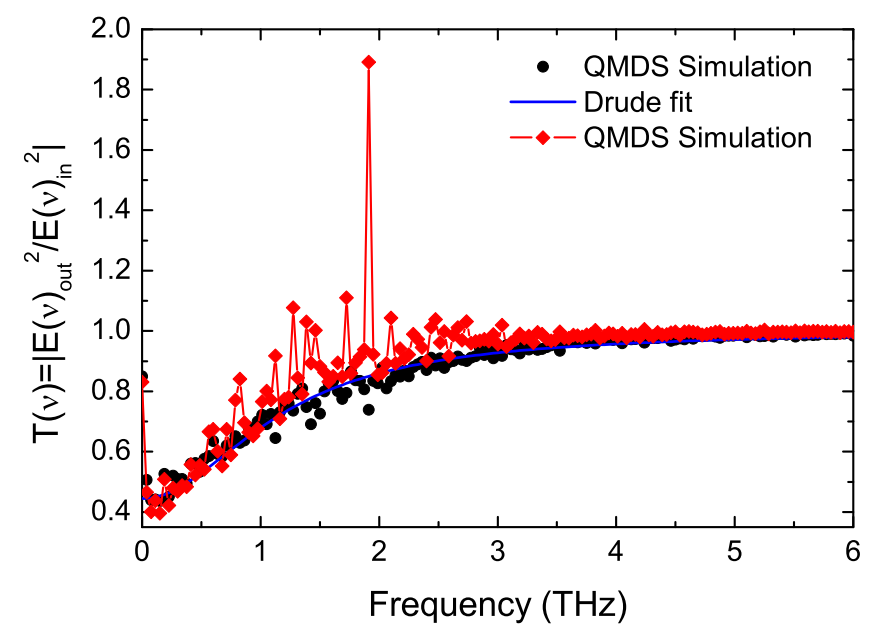

Figure 4.12: Transmission spectrum calculated with the QMDS model (30 impurities in a rectangular box with a side length of $67 \mathrm{~nm}$, corresponding to sample HD) for an equilibrium occupation of the impurity states (black dots) and for a non-equilibrium occupation, e.g. after excitation with a strong $\mathrm{THz}$ pulse (red diamonds). A transmission spectrum calculated with the Drude model assuming a scattering time of $\tau=150$ fs (blue solid line).

only very few impurities it does provide a much smaller statistical averaging over the ensemble of impurities as the experiment. Surprising is the excellent agreement between the experimental observation and the calculation with the QMDS model. However, to accurately reproduce the experimental result, the QMDS model should include a greater number of impurities in a greater volume. The resulting calculation expense would be enormous, because the calculation time rises with the third power of the volume. Thus, the discussion is restricted to the present model.

Figure 4.13 shows the transmission spectrum of a system which corresponds to sample LD (red Diamonds). For comparison, the spectrum of an isolated impurity (black dotted line) is also shown. The transmission spectrum for sample LD already shows a blueshift of the peaks in the spectrum compared to the spectrum of the isolated impurity. The spectral shift results from a delocalization of the ground state wavefunction. However, this blueshift is less pronounced than for sample HD. The QMDS model estimates that the nonlinear signal is also present for sample LD, which could not be verified in the experiment. However, as the electric field emitted from the sample is proportional to the density of initially neutral impurities, the 


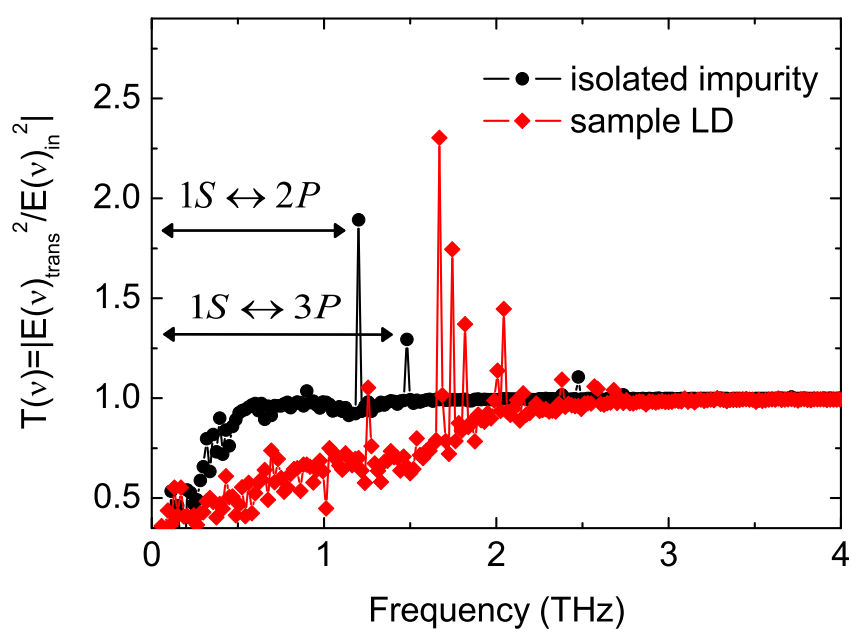

Figure 4.13: Transmission spectra calculated with the QMDS model for an impurity density which corresponds to sample LD (red Diamonds) and an isolated impurity (black dotted line).

emitted signal from sample LD is 15 times weaker compared to sample HD, as shown in Figure 2.5. The amplitudes of the observed coherent signals in sample HD (see Figure 4.5 and 4.7) are in the order of $10 \mathrm{kV} / \mathrm{cm}$. Thus signals emitted from sample LD are below the noise level of the experiment. 


\section{Chapter 5}

\section{Rabi flopping on radiatively coupled shallow donor transitions}

In this chapter the study of electrons in impurity potentials is extended to lower $\mathrm{THz}$ intensities at low temperatures. The experimental setup was built in a vacuum chamber to be able to cool the sample down to temperatures of approximately $100 \mathrm{~K}$.

Excitation of the sample with coherent $\mathrm{THz}$ pump pulses revealed Rabi oscillations in the carrier wave regime on transitions between bound impurity levels. The impurity transition is approximated by a two-level system. The sample response is analyzed with the help of model calculations which utilize the coupled Maxwell-Bloch equations.

\subsection{THz transmission spectroscopy in vacuum}

The experimental setup is depicted in Figure 5.1. The whole propagation path of the $\mathrm{THz}$ radiation was built in a vacuum chamber in order to avoid spurious signals from vapor absorption. In addition the vacuum chamber allows to approach the cooled sample with mirrors having a short focal length. $800 \mathrm{~nm}$ NIR pulses provided by a Ti:sapphire oscillator and amplified in a chirped-pulse amplifier (CPA) with a duration of $30 \mathrm{fs}$ are used as pump pulses for $\mathrm{THz}$ generation via optical rectification in a $1 \mathrm{~mm}$ thick GaSe crystal. The NIR pump pulses enter the vacuum chamber through a $3 \mathrm{~mm}$ thick glass window with a diameter of $40 \mathrm{~mm}$. The window dispersion is pre-compensated in the compressor stage of the CPA. At the sample position the generated $\mathrm{THz}$ beam is focused and collimated using two parabolic 


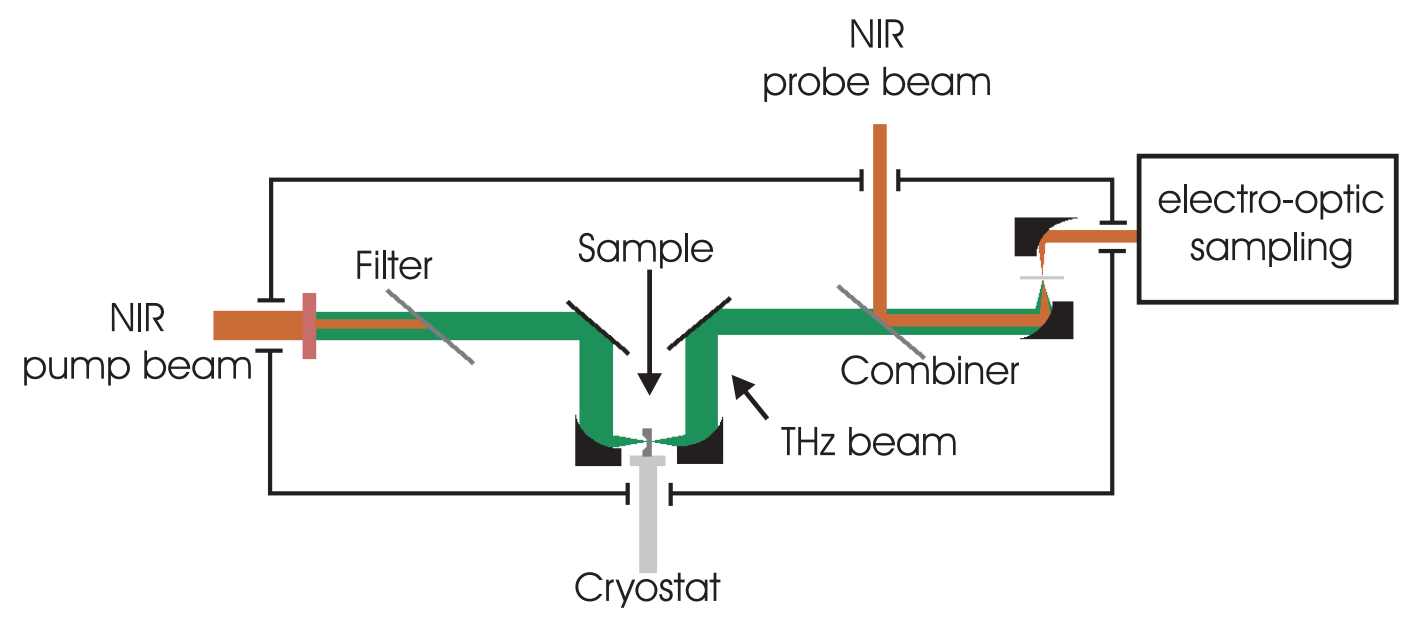

Figure 5.1: Setup for coherent $\mathrm{THz}$ propagation experiments build in a vacuum chamber. The $\mathrm{THz}$ radiation which is generated using optical rectification in a GaSe crystal is measured via electro-optic sampling in a ZnTe crystal.

mirrors with a focal length of $12.7 \mathrm{~mm}$. The transmitted $\mathrm{THz}$ radiation is overlapped with the $800 \mathrm{~nm}$ NIR probe pulse from the oscillator and measured via electro-optic sampling in a ZnTe crystal. The NIR probe pulse enters the vacuum chamber through a $300 \mu \mathrm{m}$ thick boron silica window which adds only negligible dispersion to the pulses and therefore does not affect the bandwidth of the detector.

The sample was mounted in a closed cycle helium cryostat. The cold finger was attached to the vacuum chamber via a flexible aluminum bellow which allowed for spatial adjustment of the sample from the outside of the chamber. The lowest pressure reached in the chamber was approximately $10^{-6}$ mbar and the lowest temperature reached was approximately $60 \mathrm{~K}$.

\subsection{Experimental results}

Exemplary $\mathrm{THz}$ transients are presented in Figure 5.2 (b), where the electric field of the incident $\mathrm{THz}$ pulse $\mathrm{E}_{\mathrm{in}}(t)$ (blue dashed line) and the transmitted field $\mathrm{E}_{\text {trans }}(t)$ (black dash-dotted line) are shown together with the difference signal $\mathrm{E}_{\text {trans }}(t)-\mathrm{E}_{\text {in }}(t)$ (red solid line). The measurement geometry is depicted in Figure 5.2 (a). As the sample of $d=500 \mathrm{~nm}$ thickness is much thinner than the $\mathrm{THz}$ wavelength, all carriers in the sample experience the same driving field $\mathrm{E}_{\mathrm{loc}}(t)$ which equals $\mathrm{E}_{\text {trans }}$ (see Section 3.4). Hence, the difference between the incident and the transmitted field corresponds to the field $\mathrm{E}_{\mathrm{em}}(t)$ which is coherently emitted from the sample. Since a thin film emits in both 

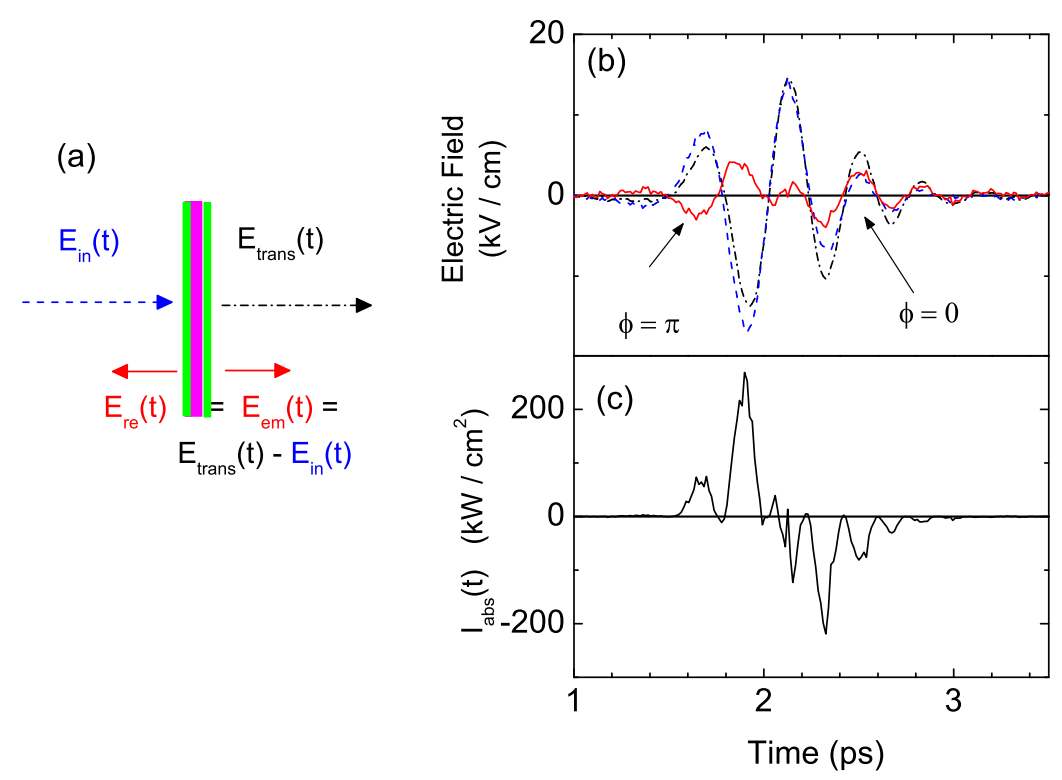

Figure 5.2: (a) Nonlinear propagation of a high field $\mathrm{THz}$ transient through an n-type GaAs layer. The extraction of the emitted field of the sample $\mathrm{E}_{\text {em }}(\mathrm{t})=\mathrm{E}_{\text {trans }}(\mathrm{t})-\mathrm{E}_{\text {in }}(\mathrm{t})$ is shown. (b) Electric field plotted as a function of time for incident pulses $E_{\text {in }}(t)$ (blue dashed line) and $E_{\text {trans }}(t)$ (black dash-dotted line). The time-dependent phase relation between $\mathrm{E}_{\mathrm{em}}(\mathrm{t})$ and $\mathrm{E}_{\text {trans }}(\mathrm{t})$ indicates absorptive $(\phi=\pi)$ and stimulated-emission-like $(\phi=0)$ phases of the sampleTHz-field interaction. (c) Time dependent energy absorption of the sample $I_{\text {abs }}(t)=-2 \epsilon_{0} c E_{\text {em }}(t) E_{\text {trans }}(t)$

directions, the reflected field is equal to the emitted field $\mathrm{E}_{\mathrm{re}}(t)=\mathrm{E}_{\mathrm{em}}(t)$.

For the analysis of the data it is instructive to consider the relative phase of the emitted field. In the first half of the incident pulse shown in Figure $5.2(\mathrm{~b}), \mathrm{E}_{\mathrm{em}}(\mathrm{t})$ oscillates in opposite phase relative to $\mathrm{E}_{\mathrm{in}}(\mathrm{t})(\phi=\pi)$, implying an energy flow from the $\mathrm{THz}$ field to the sample, i.e., absorption. In contrast, at the end of the pulse, one can observe an in-phase oscillation of $\mathrm{E}_{\mathrm{em}}(\mathrm{t})$ relative to $\mathrm{E}_{\text {in }}(\mathrm{t})(\phi=0)$, which indicates an amplification of the incident pulse. Figure 5.2 (c) shows the time dependent energy absorption rate $I_{\text {abs }}(t)$

$$
I_{\mathrm{abs}}(t)=\epsilon_{0} c\left[E_{\mathrm{in}}(t)^{2}-E_{\mathrm{em}}(t)^{2}-E_{\mathrm{trans}}(t)^{2}\right]=-2 \epsilon_{0} c E_{\mathrm{em}}(t) E_{\text {trans }}(t)
$$

using $E_{\text {trans }}=E_{\text {in }}+E_{\text {em }}$. The maxima and minima of $\mathrm{I}_{\text {abs }}$ coincide with the peaks of the local driving field $\mathrm{E}_{\mathrm{loc}}(\mathrm{t})$, indicating a resonant energy exchange 
between the $\mathrm{THz}$ field and the sample. The energy absorbed in the first period of the driving pulse is almost completely emitted in the second period.

Data for lower peak amplitudes of the driving field are summarized in Figure 5.3. In particular, the amplitude of the incident field is lower than for the transient shown in Figure 5.2 and already close to the signal to noise ratio (SNR) of the detector. The incident field is again shown in Figure 5.3 (b) (blue dashed line). The emitted field $\mathrm{E}_{\mathrm{em}}$ (black solid line) from the sample shows a strictly linear response to the driving field for incident field amplitudes below approximately $1.7 \mathrm{kV} / \mathrm{cm}$ (Figure5.3 (a) and (b)). For higher incident fields (Figure 5.3 (c)-(e)) $\mathrm{E}_{\mathrm{em}}(\mathrm{t})$ shows a strongly varying temporal shape. In particular, one observes a pronounced saturation of the amplitude of the emitted field, which is limited to values $\left|\mathrm{E}_{\mathrm{em}}(\mathrm{t})\right| \leq 0.5 \mathrm{kV} / \mathrm{cm}$. During the excitation pulse, the emission transients in Figure 5.3 (c)-(e) show fast oscillating features, which will be addressed specifically at the end of Section 5.3. The emitted field also shows coherent oscillations well after the driving pulse ( $\mathrm{t}>2 \mathrm{ps}$ ), pointing to a collective behavior of the emitting dipoles. This behavior is fully reproduced by model calculations discussed in Section 5.3.

For selected transients, the energy per electron $\Lambda(t)$ that is transiently stored in the sample is calculated:

$$
\Lambda(t)=\frac{1}{n_{d} d} \int_{-\infty}^{t} I_{\mathrm{abs}}\left(t^{\prime}\right) d t^{\prime}
$$

The energy absorption rate $\mathrm{I}_{\mathrm{abs}}(\mathrm{t})$ (black solid line) and the transiently stored energy $\Lambda(\mathrm{t})$ (black solid line) is plotted in Figure 5.4 (a), (c), (e) and Figure 5.4 (b), (d), (f), respectively. The red lines are results from model calculations which will be discussed below. In the linear case (Figure 5.4 (a), (b)) the sample shows absorptive behavior. The negative values in $I_{a b s}(t)$ and the strong oscillations in $\Lambda(\mathrm{t})$ originate from a slightly off-resonant excitation which leads to an increase of the Rabi frequency. For higher driving fields one observes an increase of the deposited energy in the sample during the first part of the driving pulse. The stored energy per electron is coherently transferred to the driving field in the second part of the $\mathrm{THz}$ driving pulse. In consequence one first observes a rise in the $\Lambda(t)$ signal, followed by a decrease close to zero at the end of the driving pulse. In Figure 5.4 (b) and $(d)$, the energy deposited per electron stays below the transition energy, i.e., $\Lambda(t=1.25 \mathrm{ps})=2.7 \mathrm{meV}<\hbar \omega_{1 S-2 P}=5.5 \mathrm{meV}$. The transition energy is marked by the dotted line in Figure 5.4 (b)-(f). However, for an incident field of $5.6 \mathrm{kV} / \mathrm{cm}$, the measurement reveals a transiently stored energy which is higher than the transition energy, indicating a breakdown of the two level 


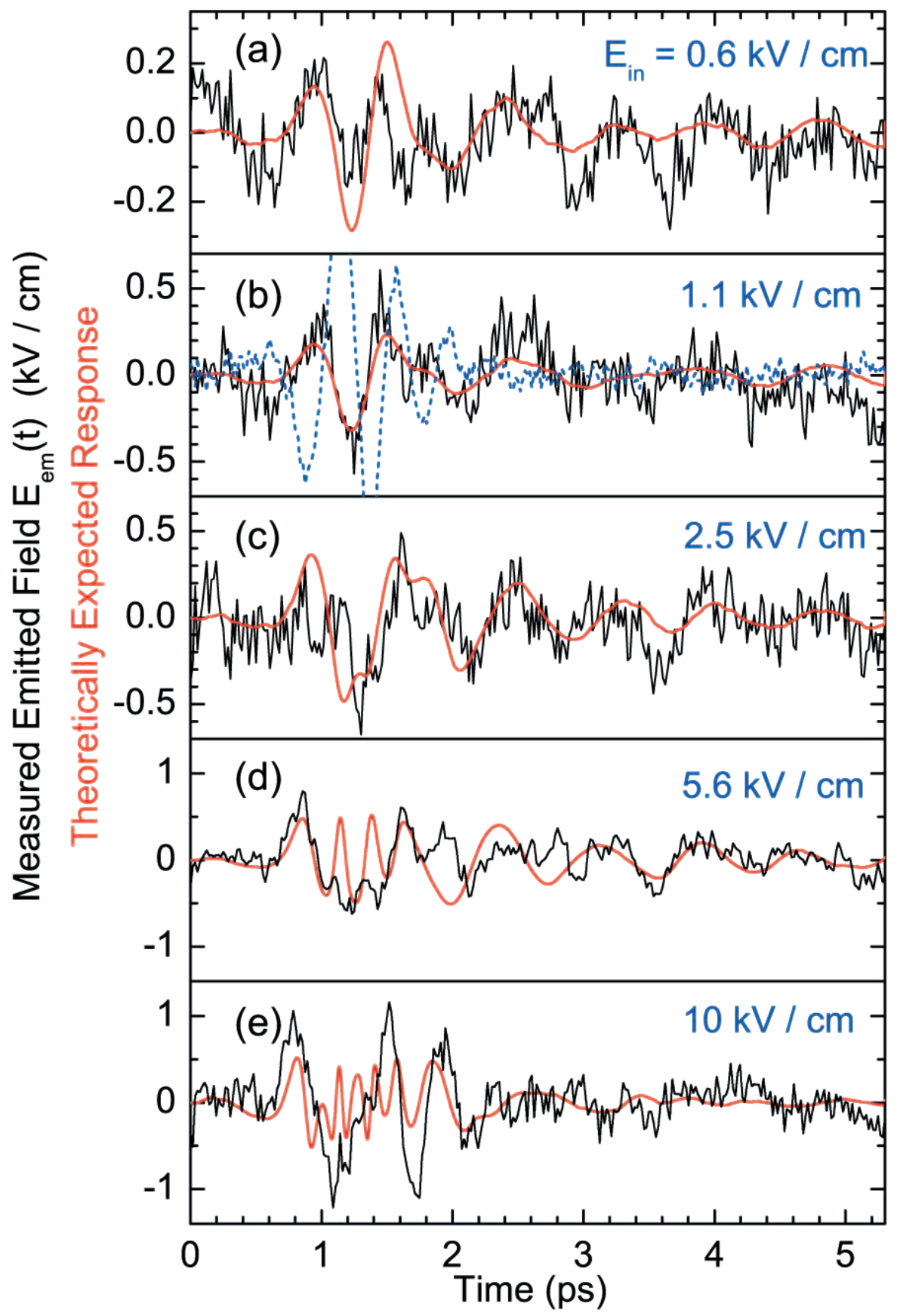

Figure 5.3: Measured emitted field transients $\mathrm{E}_{\mathrm{em}}(\mathrm{t})=\mathrm{E}_{\text {trans }}(\mathrm{t})-\mathrm{E}_{\text {in }}(\mathrm{t})$ (black solid line) for different amplitudes of the incident $\mathrm{THz}$ field $\mathrm{E}_{\text {in }}(\mathrm{t})$ and model calculations for radiatively coupled two level systems on the basis of the Bloch equations (red solid line). In (b) the incident field $\mathrm{E}_{\text {in }}(\mathrm{t})$ (blue dashed line) is shown for completeness. 


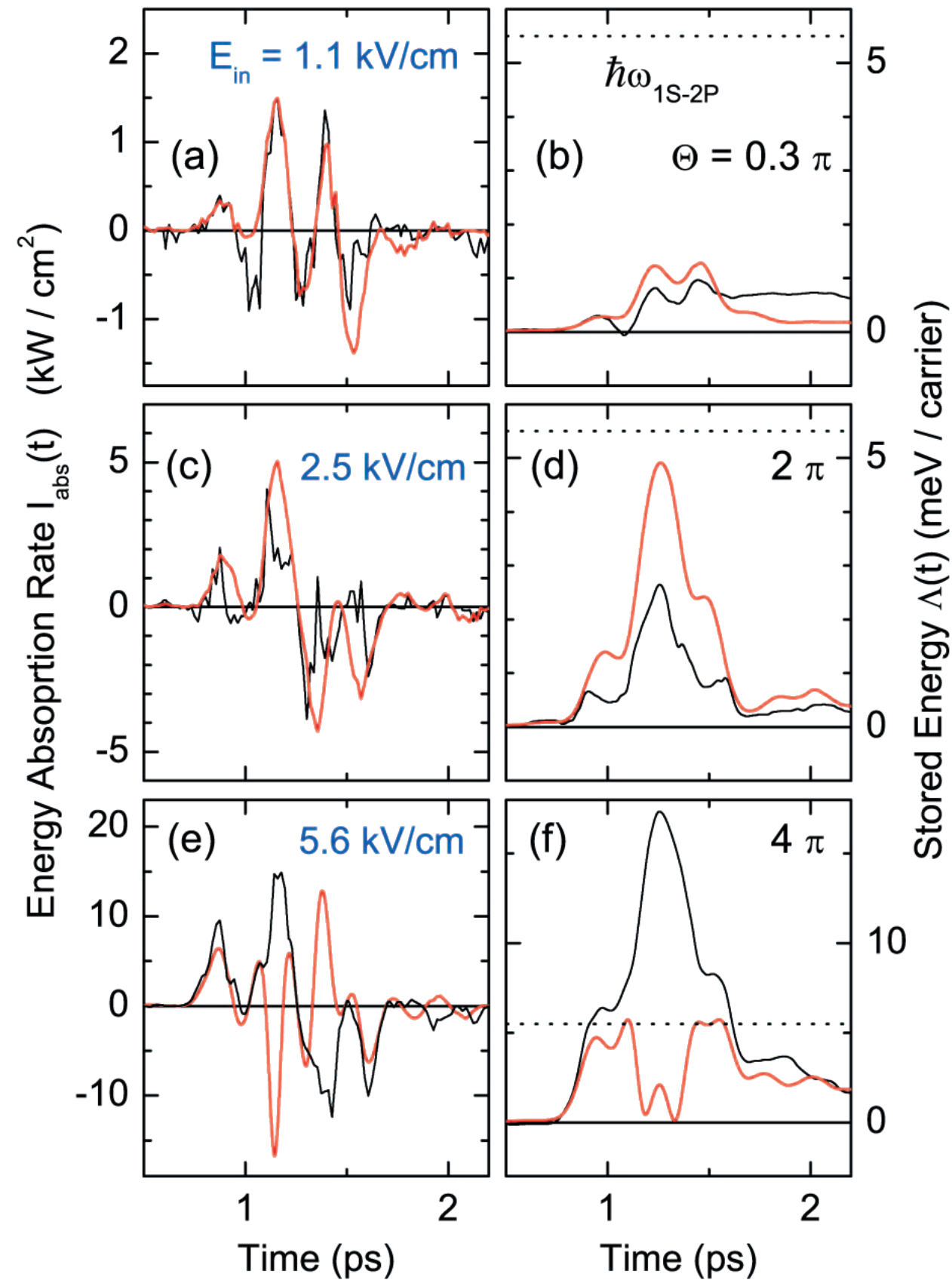

Figure 5.4: Left panels: time dependent intensity $\mathrm{I}_{\mathrm{abs}}(\mathrm{t})$ (black solid line) of the coherent energy transfer between the sample and the $\mathrm{THz}$ field corresponding to Figure 5.3 (b), (c) and (d), respectively. Right panels: corresponding transiently stored energy per electron $\Lambda(\mathrm{t})$. Model calculations using the coupled MaxwellBloch equations for a two level system are shown (red solid line) and the pulse area of the driving pulse $\Theta=0.3 \pi, 2 \pi$ and $4 \pi$ are indicated in (b), (d) and (f), respectively, as well as the assumed transition energy $\hbar \omega_{1 S-2 P}=5.5 \mathrm{meV}$. 


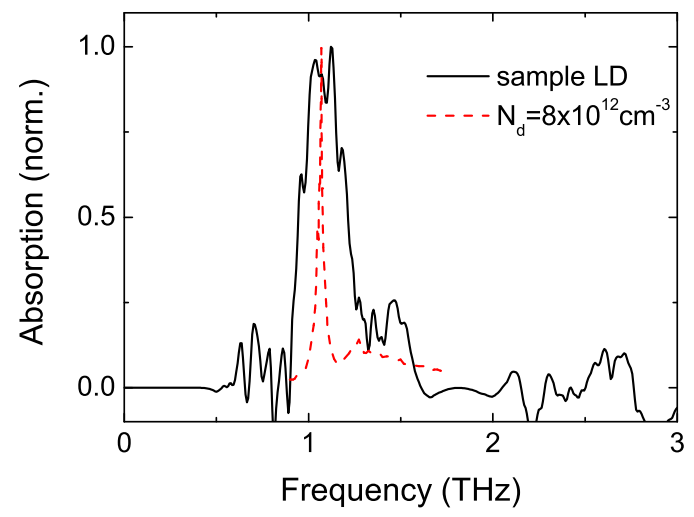

Figure 5.5: Linear absorption spectrum of sample LD (black solid line) measured at a lattice temperature of $100 \mathrm{~K}$. It is compared to a photocurrent measurement on a n-type GaAs sample with a doping concentration of $\mathrm{N}_{d}=8 \times 10^{12} \mathrm{~cm}^{-3}$ (red dashed line) taken from [32].

model. In consequence this behavior is not reproduced in the model calculations (red lines).

\subsection{Shallow donors as two level systems}

At very low doping concentrations $\left(\mathrm{N}_{d} \leq 10^{15} \mathrm{~cm}^{-3}\right)$ and at low temperatures, studies of optical transitions between bound electron states have revealed narrow transition linewidths which correspond to nanosecond population lifetimes and picosecond decoherence times $[37,185,46]$. With increasing doping concentration, the surrounding potential of a donor electron is modified by both the potentials of neighboring impurity atoms and their donor electrons. Figure 5.5 compares the linear absorption spectrum of sample LD (black solid line) to an absorption spectrum measured in a sample having a doping concentration of $\mathrm{N}_{d}=8 \times 10^{12} \mathrm{~cm}^{-3}$ (red dashed line) [32]. The absorption spectrum of sample LD was measured at a lattice temperature of $100 \mathrm{~K}$ in a commercial Fourier-transform spectrometer optimized for $\mathrm{THz}$ frequencies. The linear absorption spectra indicate discrete transitions which are slightly broadened compared to the lower doping concentration, a result which was already predicted by the QMDS model discussed in Section 4.3.2.

At a lattice temperature of $100 \mathrm{~K}$ the thermal energy of the electrons is $8.6 \mathrm{meV}$, which yields a probability of the donor ground state occupation for sample LD of $40 \%$ (see Figure 2.5). The ionization field strength of the 
impurity ground states can be estimated to $6 \mathrm{kV} / \mathrm{cm}$. For excitation fields below the ionization threshold, the electron stays confined in the impurity potential, which will be approximated by an ensemble of two level system, where the lower energy level corresponds to the 1S impurity ground state and the upper level corresponds to the $2 \mathrm{P}$ excited state. The high dipole moment of an isolated impurity favors the coherent interaction of the $\mathrm{THz}$ pulse with the ensemble, as described by the Maxwell-Bloch equations discussed in Section 2.1.4.

The quasi two-dimensional sample geometry leads to a strong radiative coupling of the carriers, which all experience the same electric field. Therefore, the driving field in equation (2.10) is in fact the local field $\mathrm{E}_{\mathrm{loc}}(\mathrm{t})$ as given by equation (3.21). The emitted field reads:

$$
E_{\mathrm{em}}=-\frac{1}{2 \epsilon_{0} c} \frac{\partial P}{\partial t}
$$

where $P$ stands for the macroscopic polarization of the ensemble of two level systems and can be obtained from the off-diagonal density matrix components

$$
P=2 N_{d} \vec{d}_{1 S \rightarrow 2 P} d R e\left(\varrho_{12}\right) .
$$

It is instructive to derive the equation of motion of the polarization component $\operatorname{Re}\left(\varrho_{12}\right)$ (see Appendix D). In the linear small signal regime $\left(\varrho_{22}=0\right)$, the result reads:

$$
\begin{aligned}
\frac{\partial^{2} \operatorname{Re}\left(\varrho_{12}\right)}{\partial t^{2}} & =\left(-\omega_{0}^{2}+\frac{1}{T_{2}^{2}}\right) \operatorname{Re}\left(\varrho_{12}\right) \\
& -\left(\frac{2}{T_{2}}+\omega_{0} \frac{N_{d} \vec{d}_{1 S \rightarrow 2 p}^{2} d}{\hbar \epsilon_{0} c}\right) \frac{\partial R e\left(\varrho_{12}\right)}{\partial t}+\omega_{0} \frac{\vec{d}_{1 S \rightarrow 2 p}}{\hbar} E_{\mathrm{in}}(t) .
\end{aligned}
$$

Hence, in the case of strong radiative coupling the damping term $\partial \varrho_{12} / \partial t$ is modified by the factor

$$
\omega_{0} \frac{N_{d} \vec{d}_{1 S \rightarrow 2 p}^{2} d}{2 \hbar \epsilon_{0} c}=\Gamma_{\mathrm{rad}}
$$

which corresponds to an inverse time constant. For a dipole moment $\vec{d}_{1 S-2 P}=$ $e \times 7.5 \mathrm{~nm}$ and a transition frequency $\omega_{0} / 2 \pi=1.3 \mathrm{THz}$ one finds $\Gamma_{\mathrm{rad}}^{-1}=6 \mathrm{ps}$. Since even the linear curve in Figure 5.3 (a) still shows oscillations more than 6 ps after the THz excitation pulse (not shown in Figure 5.3), the dephasing time constant $\mathrm{T}_{2}$ must be longer than $6 \mathrm{ps}$, since in the case of population inversion, the dephasing time constant $\Gamma_{\text {rad }}^{-1}$ counteracts the dephasing time $\mathrm{T}_{2}$. 

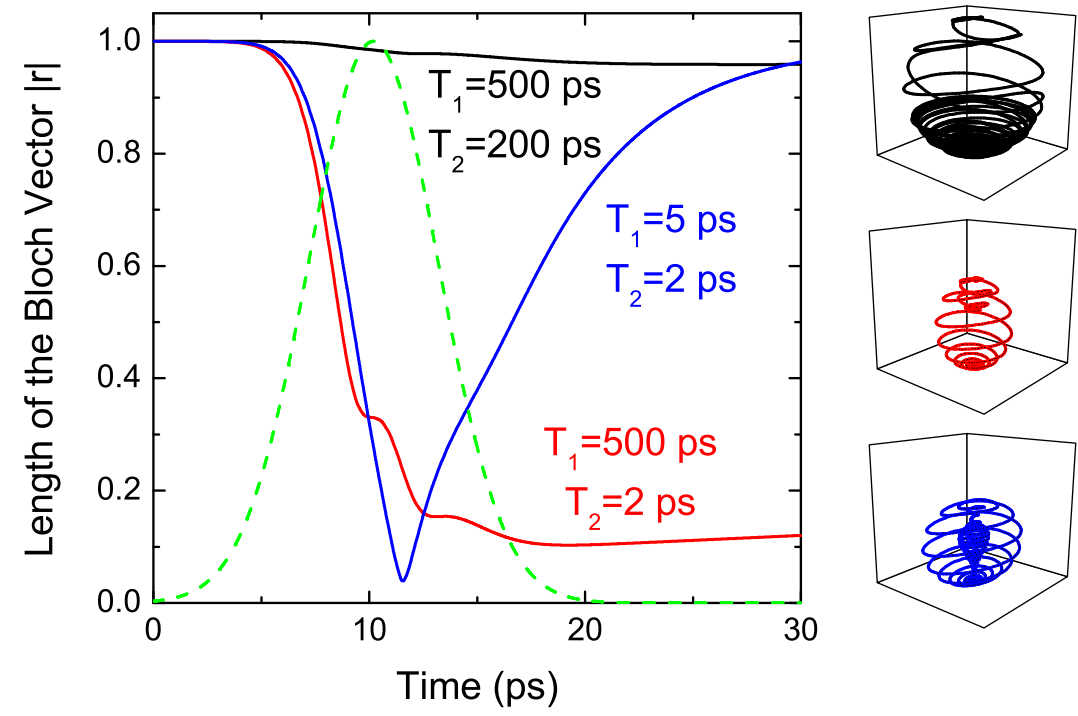

Figure 5.6: Bloch vector vs. time for three different relaxation and dephasing time constants $T_{1}$ and $T_{2}$, respectively: $T_{1}=500$ ps and $T_{2}=200 \mathrm{ps}$ (black line), $\mathrm{T}_{1}=500 \mathrm{ps}$ and $\mathrm{T}_{2}=2 \mathrm{ps}$ (red line), $\mathrm{T}_{1}=5 \mathrm{ps}$ and $\mathrm{T}_{2}=2 \mathrm{ps}$ (blue line). The envelope of the excitation pulse is plotted in the green dotted line. On the right side, the corresponding trajectory of the Bloch vector is plotted.

The influence of the dephasing and relaxation time constants is now discussed using the Bloch vector introduced in Section 2.1.4. The magnitude, i.e., the length of the Bloch vector of a two level system during the interaction with a THz pump pulse is plotted over time in Figure 5.6. The two-level system was excited with a $5 \mathrm{ps} \mathrm{THz}$ pulse with a maximum field amplitude of $1 \mathrm{kV} / \mathrm{cm}$. The relatively low electric field prevents Rabi-flopping in the carrier-wave regime. The relaxation and dephasing time constants were set to $\mathrm{T}_{1}=500 \mathrm{ps}$ and $\mathrm{T}_{2}=200 \mathrm{ps}$ (black line), $\mathrm{T}_{1}=500 \mathrm{ps}$ and $\mathrm{T}_{2}=2 \mathrm{ps}$ (red line) and $\mathrm{T}_{1}=5$ ps and $\mathrm{T}_{2}=2 \mathrm{ps}$ (blue line), respectively. If both time constants are very long, the Bloch vector shows almost no decay. This corresponds to a trajectory of the Bloch vector on the surface of the Bloch sphere, as shown in the upper right Bloch sphere in Figure 5.6. A short dephasing time leads to a decay of the Bloch vector, as shown by the red line in Figure 5.6. In the corresponding Bloch sphere, the Bloch vector moves towards the center point of the sphere, which corresponds to a remaining population of the upper level without a macroscopic polarization in the medium. If both the 


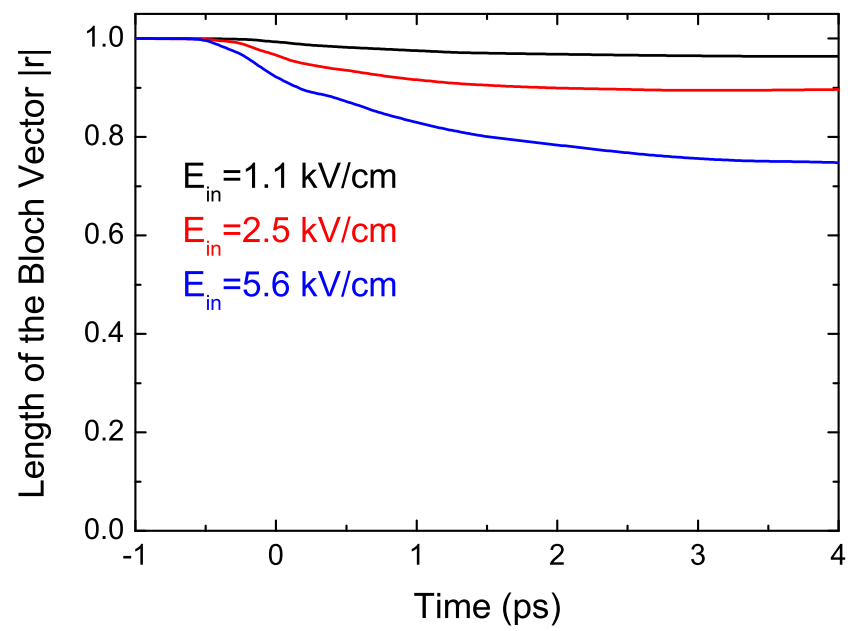

Figure 5.7: Bloch vector vs. time extracted from model calculations for excitation field strengths of $1.1 \mathrm{kV} / \mathrm{cm}$ (black line), $2.5 \mathrm{kV} / \mathrm{cm}$ (red line) and $5.6 \mathrm{kV} / \mathrm{cm}$ (blue line), respectively.

relaxation and the dephasing time constants are short, as shown by the blue line in Figure 5.6, the Bloch vector first shows a strong decay, followed by a slower increase until it saturates at the maximal length of one. This behavior can be understood by studying the lower right trajectory of the vector in the Bloch sphere. Since the dephasing time constant is at least half as long as the relaxation time constant, the Bloch vector first approaches the center of the Bloch sphere. The population relaxation, which happens with a slower time constant, leads to an increase of the magnitude of the Bloch vector due to electron relaxation in the lower state, where the length of the Bloch vector is one.

In the following, the linear and nonlinear data is modeled in an ensemble of two-level systems by numerically solving the Maxwell-Bloch equations (2.10) and (2.11) with the finite difference method in the time domain. Results of the model calculation are plotted in Figure 5.3 (red solid line) together with the experimental data. The length of the Bloch vector extracted from the model calculation is plotted in Figure 5.7 for excitation field strengths of $1.1 \mathrm{kV} / \mathrm{cm}$ (black line), $2.5 \mathrm{kV} / \mathrm{cm}$ (red line) and $5.6 \mathrm{kV} / \mathrm{cm}$ (blue line), respectively. All three curves resemble the black solid curve in Figure 5.6, i.e., the case where the interaction time is much shorter than the dephasing and relaxation time constant. In the simulations shown in Figure 5.3 and 5.7, 
the dephasing time constant was set to $6 \mathrm{ps}$, i.e., $\mathrm{T}_{2}^{-1}=\Gamma_{\text {rad }}$. The relaxation time constant $\mathrm{T}_{1}$ was set to $10 \mathrm{ps}$.

It should be noted that $T_{1}$ and $T_{2}$ were hold constant in the simulation. Hence, no fitting parameters were used in the modeling of the data. The ensemble of two level systems scales surprisingly well with the experimentally observed intensity dependance of the emitted field of the sample, until the ionization threshold is reached (Figure $5.3(\mathrm{a}-\mathrm{c})$ ). The maximal amplitude of the expected emitted field reads

$$
E_{\mathrm{em}}(\omega)=\frac{N_{d} \omega \vec{d}_{1 S \rightarrow 2 P} d}{2 \epsilon_{0} c} \approx 200 \frac{\mathrm{V}}{\mathrm{cm}}
$$

and occurs when all electrons emit coherently with the carrier frequency $\omega$.

The very good agreement of the model with the experiment can be observed in Figure 5.4, where the energy absorption rate and the transiently stored energy per carrier, which corresponds to the population of the upper level of the two level system, is represented for three different pump intensities. The model predicts the energy transfer for pulse areas up to $\Theta \approx 2 \pi$. For higher $\mathrm{E}_{\text {in }}(\mathrm{t})$ (Figure $5.4(\mathrm{e})$ and $(\mathrm{f})$ ), the temporal phase of $\mathrm{I}_{\mathrm{abs}}(\mathrm{t})$ and the amount of stored energy $\Lambda(\mathrm{t})$ are no longer described correctly by the Maxwell-Bloch equations because the two level approximation breaks down. The measured stored energy shows that electrons are excited significantly above the ionization energy of the donor. However, the energy exchange between the sample and the $\mathrm{THz}$ field is still a coherent process, implying a macroscopic polarization of the sample generated by carriers in the conduction band continuum.

\section{Rabi oscillations in the carrier wave regime}

Most experimental studies of Rabi oscillations utilized temporarily long optical driving pulses, i.e., the pulse envelope varies little over an optical oscillation period [26]. In that case, the slowly varying envelope approximation (SVEA) can be applied. Connected to that, the Maxwell-Bloch equations are often simplified by neglecting fast oscillating terms and only account for the beating of the optical frequency with the transition frequency. This is called the rotating wave approximation (RWA).

However, in the experiment discussed here both approximations are violated, since an ultrashort $\mathrm{THz}$ pulse is employed where the pulse envelope changes significantly over an oscillation period of the carrier frequency. In addition, the electric field amplitude of the driving pulse is high enough for the Rabi frequency to approach and exceed the transition frequency, leading to a breakdown of the RWA [186]. Such carrier wave (CW) Rabi oscillations 

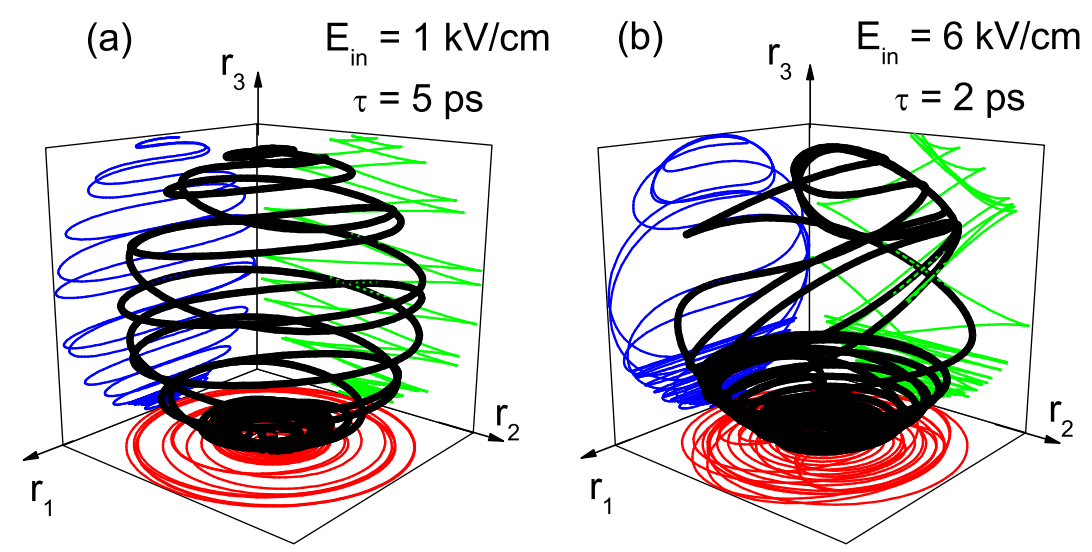

Figure 5.8: Time evolution of the Bloch-vector $\vec{r}(\mathrm{t})$ (black dotted line). The projection on the $\mathrm{x}$-y-plane (red solid line) shows a constant rotation of the induced polarization. The projections on the $\mathrm{x}$-z-plane (green solid line) and the $\mathrm{y}$-z-plane (blue solid line) show the oscillation of the absorptive and the dispersive component. For maximum coherence between the two-level systems in the ensemble, $\vec{r}(\mathrm{t})$ always moves on the surface of the Bloch sphere. (a) Rabi oscillation driven by a weak $\left(E_{\text {in }}=1 \mathrm{kV} / \mathrm{cm}\right)$ gaussian optical pulse with a temporal length of $\tau=5 \mathrm{ps}$. (b) Rabi oscillations driven by a short $(\tau=2 \mathrm{ps})$, intense $\left(\mathrm{E}_{\mathrm{in}}=6 \mathrm{kV} / \mathrm{cm}\right)$ driving pulse. The magnitude of the population component $\left(\mathrm{r}_{3}\right)$ oscillates almost as fast as the magnitude of the polarization components $\left(\mathrm{r}_{1}, \mathrm{r}_{2}\right)$, which is a signature of $\mathrm{CW}$ Rabi-flopping. Note that in particular the $\vec{r}(\mathrm{t})$ does not necessarily cross the poles of the Bloch sphere. Therefore in the CW regime, the system is not completely inverted.

were first experimentally observed by Mücke et al. [187] on the interband transition of GaAs. The authors identify the CW regime by the appearance of higher harmonics in the spectrum of the emitted signal. Such features have already been predicted in earlier theoretical studies $[188,186]$. The higher harmonic signal was applied to phase stabilization of ultrashort optical pulses $[189,190]$.

Figure 5.8 (a) and (b) depict the time evolution of the Bloch-vector $\vec{r}(\mathrm{t})$ for normal and CW Rabi oscillations, respectively. Conventional Rabi flopping driven by a gaussian optical driving pulse with an amplitude of $\mathrm{E}_{\mathrm{in}}=1 \mathrm{kV} / \mathrm{cm}$ and a temporal length of $\tau=5 \mathrm{ps}$ is shown in Figure 5.8 (a). Relaxation and dephasing time constants were set to high values, such that $\vec{r}(\mathrm{t})$ rotates on the surface of the Bloch-sphere. In the $\mathrm{CW}$ regime $\left(\mathrm{E}_{\mathrm{in}}=6 \mathrm{kV} / \mathrm{cm}\right.$ and $\tau=2 \mathrm{ps}$ ), the trajectory of the Bloch-vector also shows fast oscillations 


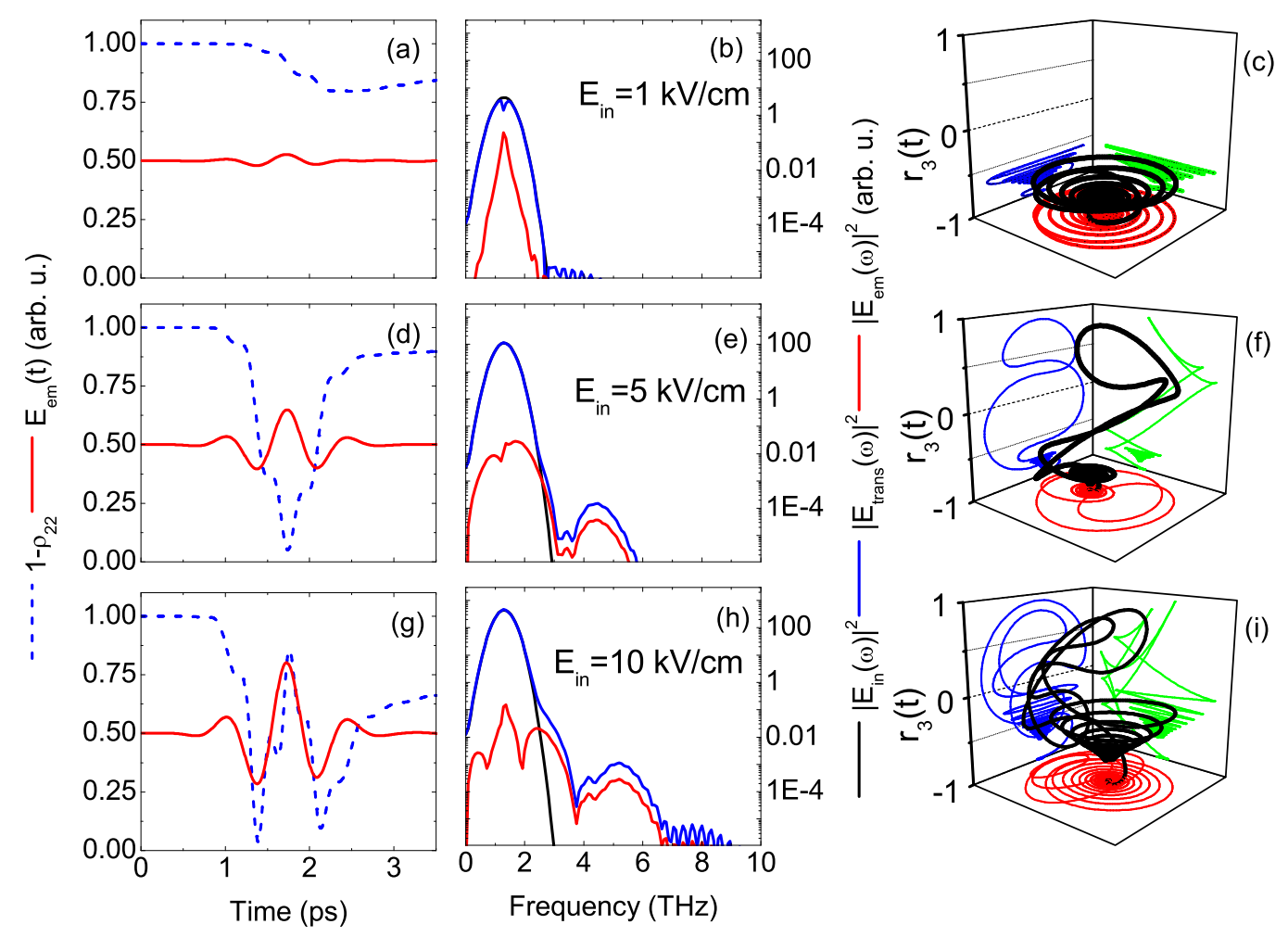

Figure 5.9: Time evolution of the population, the spectrum of the incident, transmitted and emitted pulse and the trajectory of the Bloch-vector for a gaussian driving pulse with $\tau=0.7 \mathrm{ps}$ and amplitudes $\mathrm{E}_{\mathrm{in}}=1 \mathrm{kV} / \mathrm{cm}(\mathrm{a}-\mathrm{c}), \mathrm{E}_{\mathrm{in}}=5 \mathrm{kV} / \mathrm{cm}$ (d-f) and $E_{\text {in }}=10 \mathrm{kV} / \mathrm{cm}$ (g-i). Asymmetric features in the evolution of the time dependent upper state population at the zero crossing of the driving field appear for higher driving fields. The emitted signal also shows the characteristic generation of higher harmonic components.

parallel to the $\mathrm{r}_{3}$-axis, corresponding to a fast oscillation of the population. In particular $\vec{r}(\mathrm{t})$ does not necessarily propagate through the poles of the sphere, which means that the system is not completely inverted. The projections of the Bloch sphere on the side planes (blue and green line) show the evolution of the real and imaginary part of the polarization. The projection on the $r_{3}$-plane depicts the evolution of the magnitude of the polarization.

Theoretical studies [188] showed that in the CW regime typically plateaulike features appear in the evolution of the upper state polarization expressed by the $r_{3}(t)$-component of the Bloch-vector. These features occur when the driving field $\mathrm{E}_{\mathrm{in}}$ is zero and correspond to a modulation of the $\mathrm{r}_{3}(\mathrm{t})$-component with two times the driving frequency. In conventional Rabiflopping, the modulation is very weak and is often neglected by applying 
the RWA. The only generated frequency components are side bands $(\omega-\Omega)$ and $(\omega-\Omega)$ of the carrier frequency $\omega$ which form the so-called Mollowtriplett. However, the modulation of $\mathrm{r}_{3}(\mathrm{t})$ becomes particularly prominent in the carrier-wave regime. The Optical Bloch Equation 2.11 shows that a modulation of the population modulates the nonlinear polarization $\varrho_{12}$ of the density matrix. This leads to the generation of higher frequency components of the carrier frequency in the carrier-wave regime.

The upper level occupation is measured by the transiently stored energy $\Lambda(\mathrm{t})$ (see Figure 5.4), which clearly shows plateaus at the zero-crossings of the driving field. It must be noted that these features partly steam from the non-resonant excitation of the two level system, which has a transition frequency of $1.3 \mathrm{THz}$ compared to the center frequency of the driving pulse of $2 \mathrm{THz}$. For comparison, in a simulation, an ensemble of two level systems with the parameter $\mathrm{N}_{d}=2 \times 10^{16} \mathrm{~cm}^{-3}, \mathrm{~d}=500 \mathrm{~nm}, \vec{d}_{1 S \rightarrow 2 P}=\mathrm{e} \times 7.5 \mathrm{~nm}$ and $\omega_{\text {trans }} / 2 \pi=1.3 \mathrm{THz}$ was resonantly excited with a gaussian optical pulse centered at the transition frequency, with a fixed pulse length of $\tau=0.7 \mathrm{ps}$ and varying amplitudes. These parameters correspond to a resonant excitation of sample LD. The population and dephasing time constant were set to $10 \mathrm{ps}$ and $5 \mathrm{ps}$, respectively. The time evolution of the ground state population $\left(1-\varrho_{22}\right)$, the spectrum of the incident, transmitted and emitted pulse and the trajectory of the Bloch-vector are shown in Figure 5.9 (a-c), 5.9 (d-f) and 5.9 (g-i) for $\mathrm{E}_{\mathrm{in}}=1 \mathrm{kV} / \mathrm{cm}, 5 \mathrm{kV} / \mathrm{cm}$ and $10 \mathrm{kV} / \mathrm{cm}$, respectively.

The time evolution of the ground state population clearly shows plateaus at the zero crossings of the driving field, a characteristic of CW Rabi oscillations (Figure $5.9(\mathrm{a}),(\mathrm{d})$ and $(\mathrm{g})$ ). More instructive are the spectra of the emitted signal (Figure 5.9 (b), (e) and (h)), where the appearance of higher harmonics in the emitted spectrum at growing incident field strength is shown.

For a driving field of $\mathrm{E}_{\mathrm{in}}=5 \mathrm{kV}$ shown in Figure 5.9 (e), one notes a peak in $\mathrm{E}_{\mathrm{em}}(\omega)$ around $5 \mathrm{THz}$, a frequency which can not be detected by the electrooptic sampling detector, as it corresponds to the LO-phonon resonance of the ZnTe detection crystal. Therefore this oscillation does not appear in the measurement shown in Figure 5.3 (d). However, fast oscillations are visible in the simulated transient in Figure 5.3 (d). For a driving field of $10 \mathrm{kV} / \mathrm{cm}$, the emitted spectrum stretches to even higher frequencies (Figure $5.9(\mathrm{~h})$ ) which again appears in the simulated transients shown in Figure 5.3 (e). The trajectories of the Bloch-vector also demonstrate the evolution from conventional Rabi-flopping (Figure 5.9 (c)) to the CW regime (Figure 5.9 (f) and (i)). 


\section{Chapter 6}

\section{Internal motions of a quasiparticle governing its ultrafast nonlinear response}

The interaction between a charged particle in a polar surrounding is of broad interest. Examples are a proton in ice [164], an ion in a DNA molecule [191], an electron at an interface [192] or an electron in an organic [193] or inorganic [194, 195, 196] crystal. In polar semiconductors like gallium arsenide, the polar interaction between conduction band electrons and the lattice leads to the formation of quasiparticles, the so-called Fröhlich polarons [106].

In this chapter an experimental study of the internal quasi-particle dynamics of a coupled electron-phonon system is studied via time-resolved THzpump MIR-probe spectroscopy. The impulsive excitation of conduction band electrons with an intense $\mathrm{THz}$ pulse triggers oscillations of the electron in the self consistent polaron potential which results from the electron-lattice interaction. These oscillations in turn act back on the electron. The electron motion within the lattice distortion is measured by mid-infrared probe pulses. The experimental data are first discussed qualitatively. A theoretical model which accounts for the quantum kinetic dynamics is presented at the end of this chapter.

\section{1 n-type GaAs sample and experimental setup}

The sample investigated in the nonlinear transport measurement is sample HD already discussed in Section 4.1 and shown in Figure 4.1. In contrast to previous studies of carrier transport in the quantum kinetic regime ([87] and references therein) this sample contains free conduction band carriers 


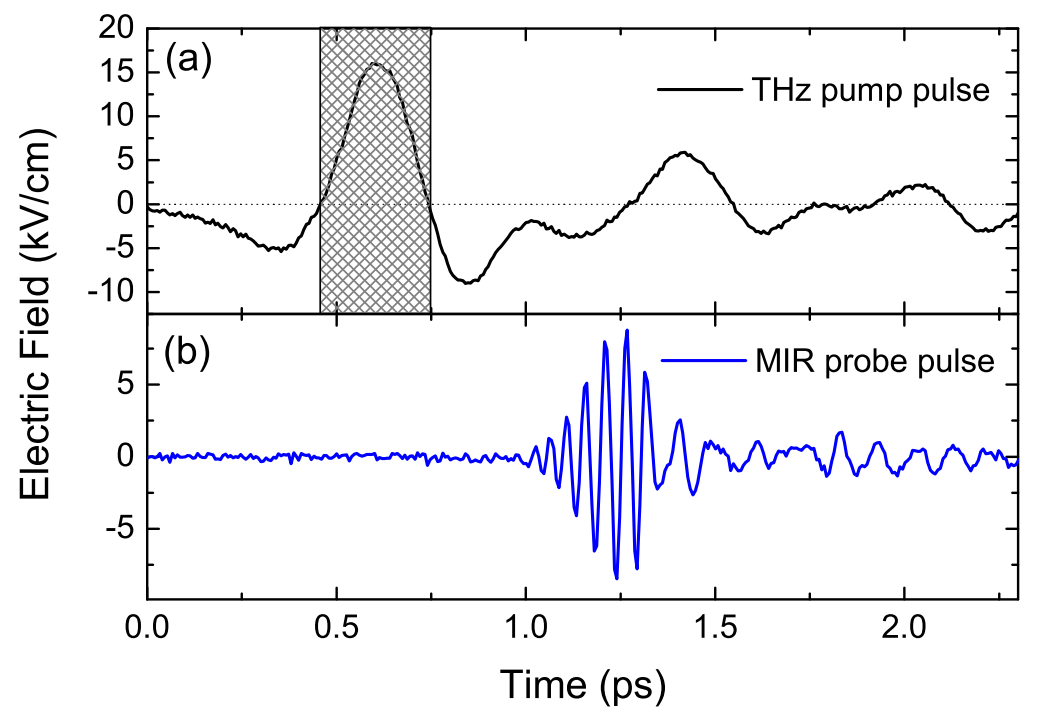

Figure 6.1: Electric field of the $\mathrm{THz}$ pump and MIR probe pulse vs. time. (a) $\mathrm{THz}$ pump pulse: The interesting part of the $\mathrm{THz}$ pulse, i.e., the region around the highest positive lobe, is marked by the gray area. (b) MIR probe pulse.

through doping. Therefore, no electron-hole pair generation is necessary and no interaction with other carrier types, e.g., electron-hole scattering, occurs. In the linear regime the sample shows a transmission spectrum which is structureless for mid-infrared frequencies.

The experimental setup was presented in Section 3.5 and is shown in Figure 3.20. The THz pump pulse which was generated by optical rectification in dry nitrogen via four wave mixing is shown in Figure 6.1 (a). In order to study field induced effects, it is advantageous to generate shaped $\mathrm{THz}$ pulses, with an unidirectional electric field. For that reason, the delay between $\mathrm{THz}$ pump and MIR probe pulse was limited to values where the MIR pulse interacts with the pump pulse in the vicinity of the shaded gray area in Figure 6.1 (a). The ultrashort MIR-pulse is generated by difference frequency mixing in a GaSe crystal and has a center frequency of $17 \mathrm{THz}$. It is shown in Figure 6.1 (b). Both the THz and the MIR pulse are generated using the same optical pump pulse as explained in Section 3.

The transmitted THz and MIR transients were detected in amplitude and phase using electro-optic sampling. Two choppers with chopper frequencies of $500 \mathrm{~Hz}$ and $125 \mathrm{~Hz}$ for the $\mathrm{THz}$ and the MIR pulses, respectively, allow 

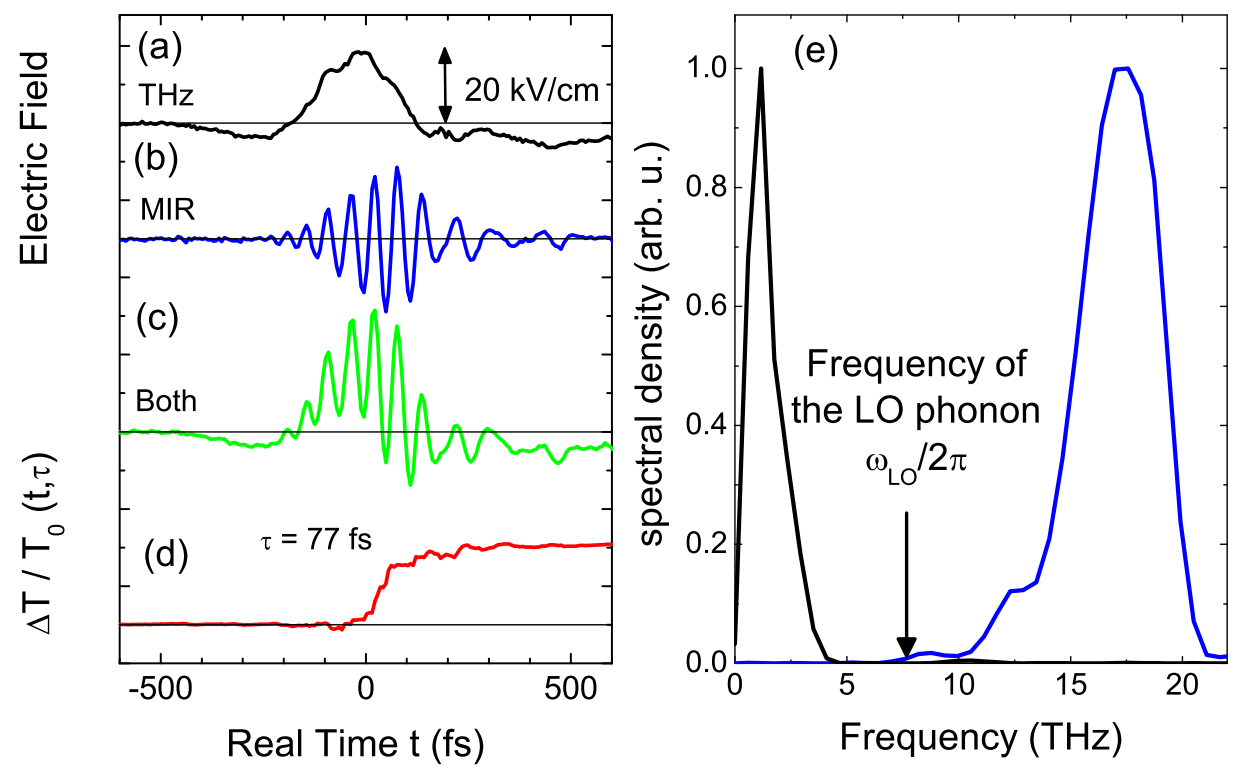

Figure 6.2: (a) $\mathrm{THz}$ pump pulse with a maximum field amplitude of $20 \mathrm{kV} / \mathrm{cm}$ (b) MIR probe pulse and (c) the combined signal measured for a relative delay of $\tau=77$ fs. (d) Steplike increase of the MIR transmission. (e) Spectra of the $\mathrm{THz}$ pulse (black solid line) and the MIR pulse (blue solid line). In addition the frequency of the LO phonon is marked.

to measure the transmitted signal with only the $\mathrm{THz}$ pulse $\left(\mathrm{E}_{\mathrm{THz}}\right)$, only the MIR pulse $\left(\mathrm{E}_{\mathrm{MIR}}\right)$ or both pulses $\left(\mathrm{E}_{\mathrm{both}}\right)$ incident on the sample.

In Figure 6.2, the electric field of the transmitted $\mathrm{THz}$ pump pulse (a), the mid-infrared probe pulse (b), and the combination of the two (c) are presented as a function of real time $t$ for a mutual delay between the pulses of $\tau=77 \mathrm{fs}$. The $\mathrm{THz}$ induced transmission change of the mid-infrared radiation can be derived using the following expression:

$$
\frac{\Delta T(\tau, t)}{T_{0}}=\frac{\int_{-\infty}^{\infty} d t^{\prime} E_{\mathrm{MIR}}\left(\tau, t^{\prime}\right) E_{\mathrm{NL}}\left(\tau, t^{\prime}\right)}{\int_{-\infty}^{\infty} d t^{\prime}\left|E_{\mathrm{MIR}}\left(\tau, t^{\prime}\right)\right|^{2}}
$$

where

$$
E_{\mathrm{NL}}=E_{\mathrm{both}}-E_{\mathrm{THz}}-E_{\mathrm{MIR}}
$$

The measurement of the independent pulses is discussed in Section 3.5. 


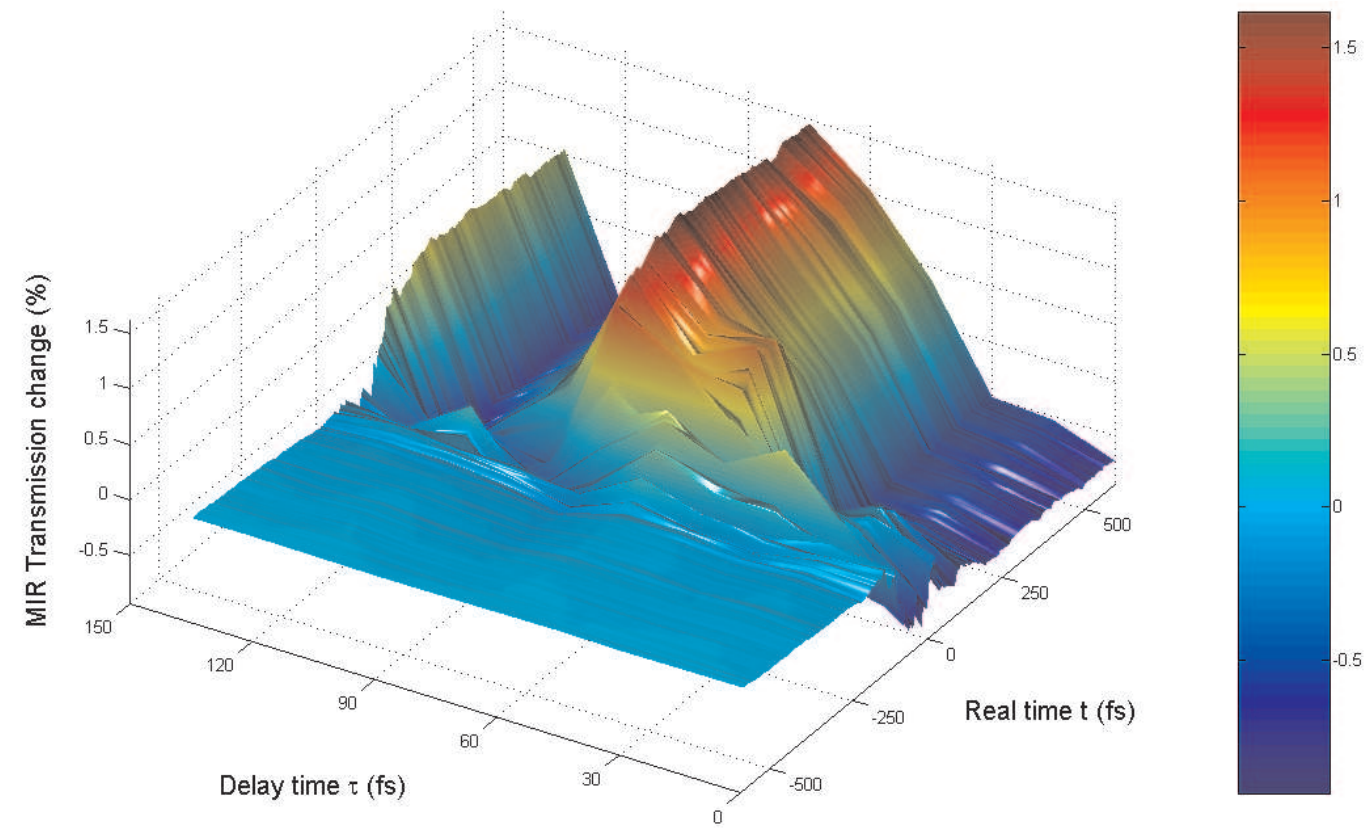

Figure 6.3: Two-dimensional plot of the mid-infrared transmission change.

The time dependent mid-infrared transmission change as calculated from equation 6.1 is shown for a pump-probe delay $\tau=77$ fs in Figure 6.2 (d). It increases in a steplike fashion while the $\mathrm{THz}$ and MIR pulses interact with the sample, and reaches a constant value after $t=250 \mathrm{fs}$. Hence, the nonlinear mid-infrared transmission change occurs in a narrow time window only. Figure 6.2 (e) shows the spectra of the THz and MIR pulse, respectively. In addition, the frequency of the LO phonon in gallium arsenide is indicated. The $\mathrm{THz}$ and the MIR pulse have no spectral density at the LO phonon resonance.

A two dimensional plot of the change in mid-infrared transmission is shown in Figure 6.3. The $\mathrm{THz}$ pump MIR probe delay is denoted by the time variable $\tau$, while the real time coordinate is denoted $t$. The delay time $\tau$ is defined by the temporal distance between the intensity maximum of the MIR pulse and the maximum of the electric field of the THz pulse. Figure 6.3 shows a steplike behavior of the transmission change along the real time coordinate. For delay times $\tau=0$ and $\tau=120$ fs one observes a transmission decrease at $\mathrm{t} \rightarrow \infty$. In contrast mid-infrared gain is observed around $\tau=75 \mathrm{fs}$ and $\tau=135$ fs.

Figure 6.4 (b) plots the mid-infrared transmission change $\Delta \mathrm{T}(\tau, \infty)$ integrated over real time $t$ as a function of the delay time $\tau$. The oscillatory behavior of the transmission change is clearly visible. Figure 6.4 (a) shows 

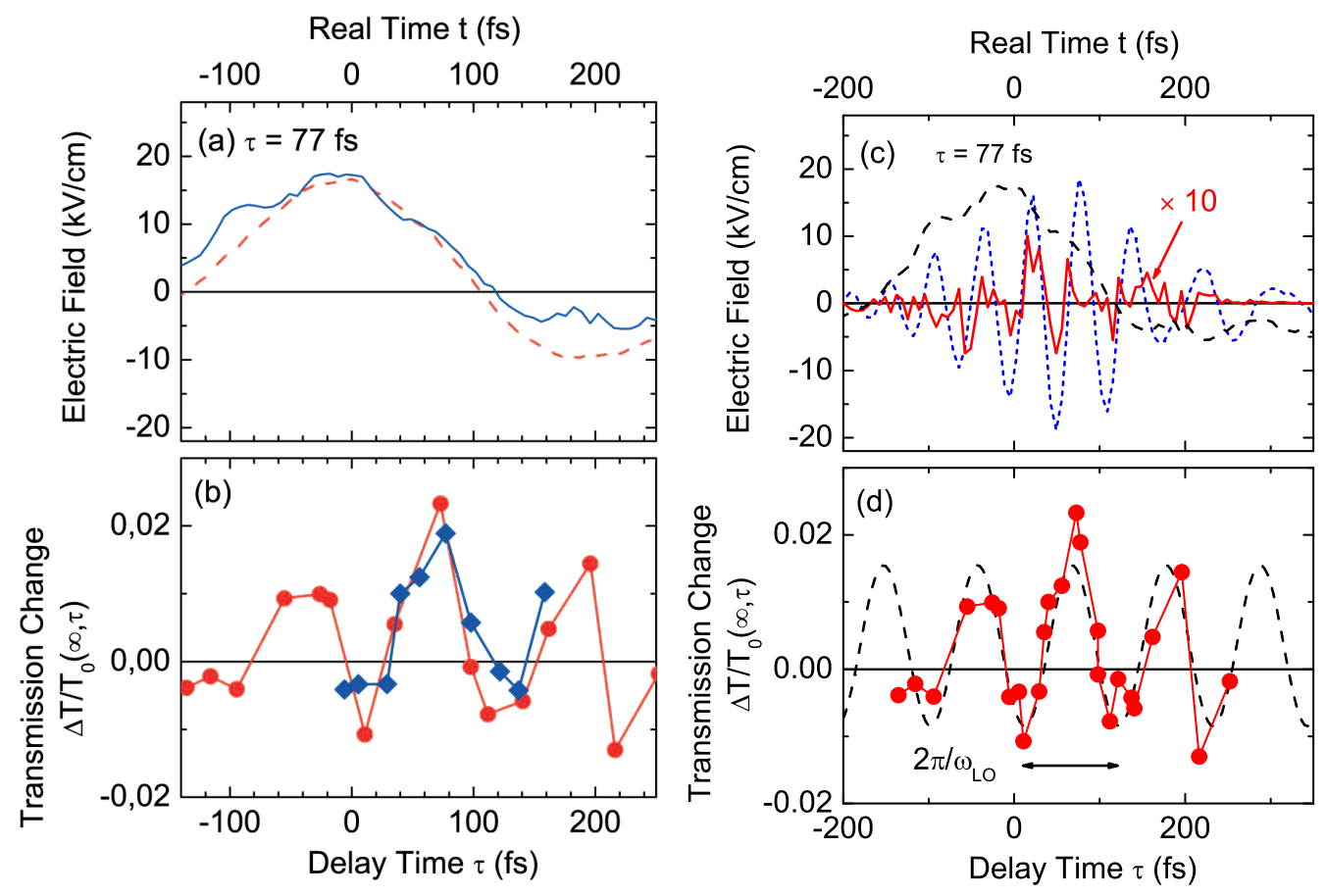

Figure 6.4: MIR transmission change at $\Delta \mathrm{T}(\tau, \infty)$ vs. the delay time coordinate $\tau$. (a) THz pump pulses of two individual measurements. (b) $\Delta \mathrm{T}(\tau, \infty)$ vs. $\tau$. the blue diamonds correspond to the blue solid THz pump pulse in (a), the red dotted measurement corresponds to the red dashed THz pump pulse in (a). (c) Sampling of the THz pump pulse (black dashed line) with the MIR probe pulse (blue short dashed line). The red solid line shows the nonlinear polarization ten times enlarged. (d) Data already shown in (a), this time compared to the oscillation of the LO phonon in gallium arsenide (black dashed line).

THz pump pulses of two independent two dimensional scans (blue solid and red dashed line, respectively). The most relevant delay times $\tau$ of the $\mathrm{THz}$ pulse correspond to the gray shaded area in Figure 6.1. The blue diamond curve in Figure 6.4 (b) was obtained after excitation with the blue solid $\mathrm{THz}$ pulse in Figure 6.4 (a) and the red dotted curve belongs to the red dashed $\mathrm{THz}$ pulse in Figure 6.4 (a). Comparing both measurements, one notes that the phase of the oscillation in $\Delta \mathrm{T}(\tau, \infty)$ is locked to the phase of the $\mathrm{THz}$ pump pulse. The oscillation of the MIR transmission change is triggered by the rising positive lobe of the $\mathrm{THz}$ pump pulse. Additional measurements on sample LD do not show oscillations in the intra-band mid-infrared absorption.

The measurement of the $\mathrm{THz}$ induced change in the mid-infrared transmission is again shown in Figure 6.4 (c). The THz pulse (black dashed line) 
is sampled by the MIR probe pulse (blue short dashed line). The resulting nonlinear polarization signal is shown in the red solid curve ten times enlarged. Figure $6.4(\mathrm{~d})$ compares the oscillation of the MIR transmission $\Delta \mathrm{T}(\tau, \infty)$ (red dotted line), which was already shown in Figure $6.4(\mathrm{~b})$, to the frequency of the LO phonon in gallium arsenide (black dashed line). The oscillation in $\Delta \mathrm{T}(\tau, \infty)$ is triggered for a $\mathrm{THz}$ field higher than $10 \mathrm{kV} / \mathrm{cm}$. For amplitudes of the driving field $\leq 20 \mathrm{kV} / \mathrm{cm}$, which is approximately the highest field strength applied in our experiments, the oscillation in the midinfrared absorption is constant and the oscillation frequency corresponds to the frequency of the LO phonon in gallium arsenide.

\subsection{Discussion}

First, the underlying physics causing the coherent oscillation of the midinfrared transmission is discussed qualitatively. It is followed by a theoretical model based on the solution of the Heisenberg equation of motion of the carrier velocity operator. This model accounts for the quantum kinetic nature of the electron propagation in the polar gallium arsenide lattice. Finally results of the model calculations are compared with the experiment.

\subsubsection{Qualitative discussion}

Since the oscillation in the mid-infrared transmission is observed on sample HD only, the effect stems from conduction band electrons introduced by doping. In thermal equilibrium, electrons in a gallium arsenide lattice are found in so-called dressed states, i.e., they form polarons due to the Fröhlichinteraction with the lattice as shown in Figure 6.5 (a) and (b). An external electric field acting on a polaron induces charge transport which is described by the drift velocity $\mathrm{v}_{d}$. For weak external fields $\mathrm{E}_{\text {ext }}$, the electron is not displaced from the polaron center and the response to the external field is determined by the center-of-mass motion of the entire quasiparticle. Assuming the electric field in $\mathrm{x}$-direction, one finds a drift motion $\mathrm{x}(\mathrm{t})=\mathrm{v}_{d} \mathrm{t}=\mu \mathrm{E}_{\text {ext }} \mathrm{t}$. Such a situation is depicted in Figure 6.5 (c) and (d). An abrupt application of a strong external field $\mathrm{E}_{\text {ext }}(t)$ leads to a displacement of the electron from the polaron center along the internal coordinate r. When the electron has gained sufficient energy to emit LO phonons, the velocity saturates. The acceleration time must be shorter than the inverse LO phonon period $\left(\omega_{L O} / 2 \pi\right)^{-1}$, i.e., faster than the response time of the lattice. Figure 6.6 shows friction free acceleration of an electron in gallium arsenide by the electric field of the $\mathrm{THz}$ pump pulse employed in the experiment. 


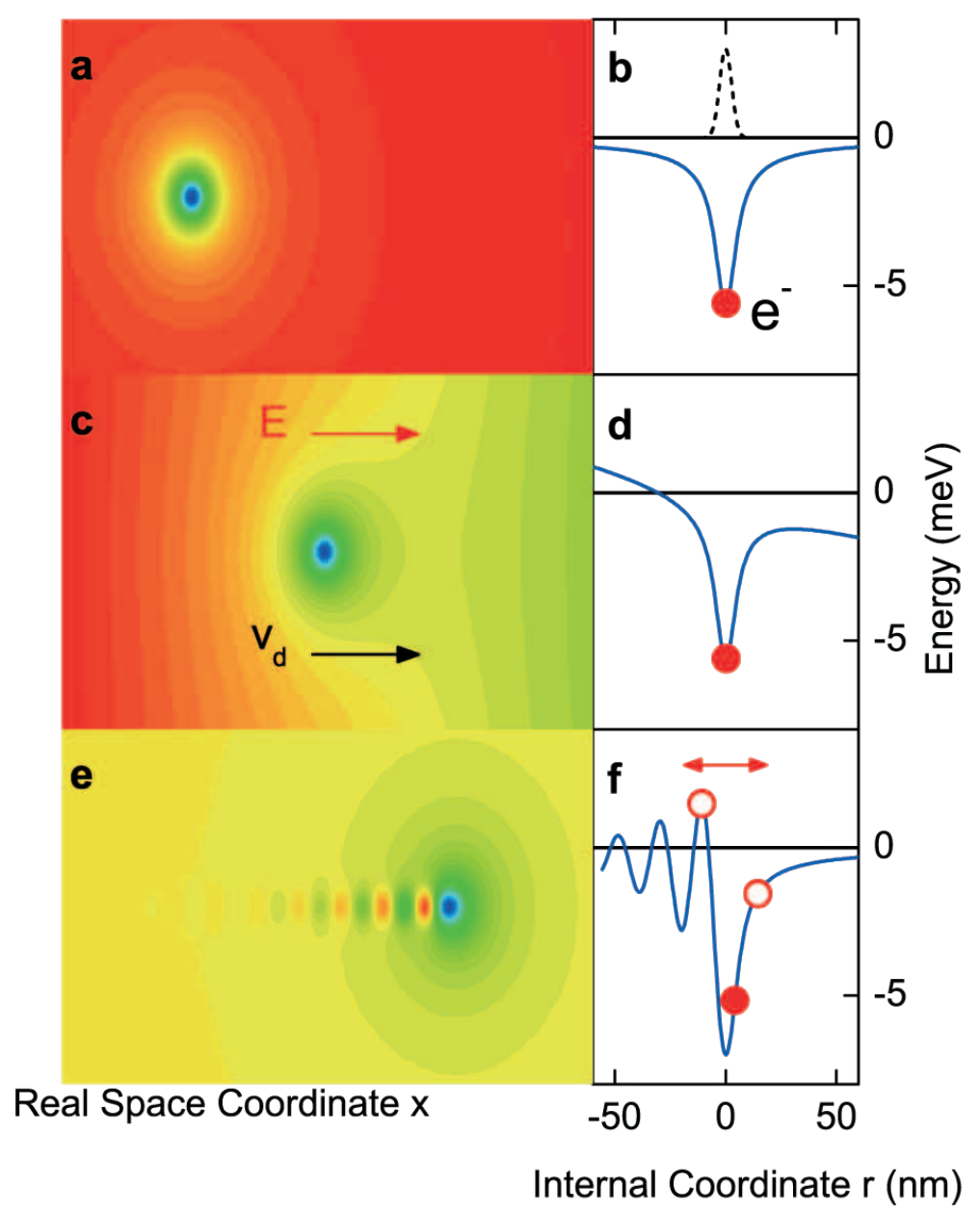

Figure 6.5: (a) Contour plot of the self-induced polaron potential in thermal equilibrium. (b) Polaron equilibrium potential (blue solid line) and electron wavefunction (black dotted line). (c), (d) Linear transport with the drift velocity $\mathrm{v}_{d}$. The total potential is the sum of the applied potential and the zero-field polaron potential. (e), (f) Nonlinear transport in a strong external field: the external potential has been subtracted. The electron (red circle in (f)) is displaced from the minimum of the LO phonon cloud and generates coherent phonon oscillations in its stern wave. The nonlinear polarization of the LO phonon cloud leads to oscillations of the electron along the internal coordinate $\mathrm{r}$, which add to the drift motion of the entire quasiparticle. 


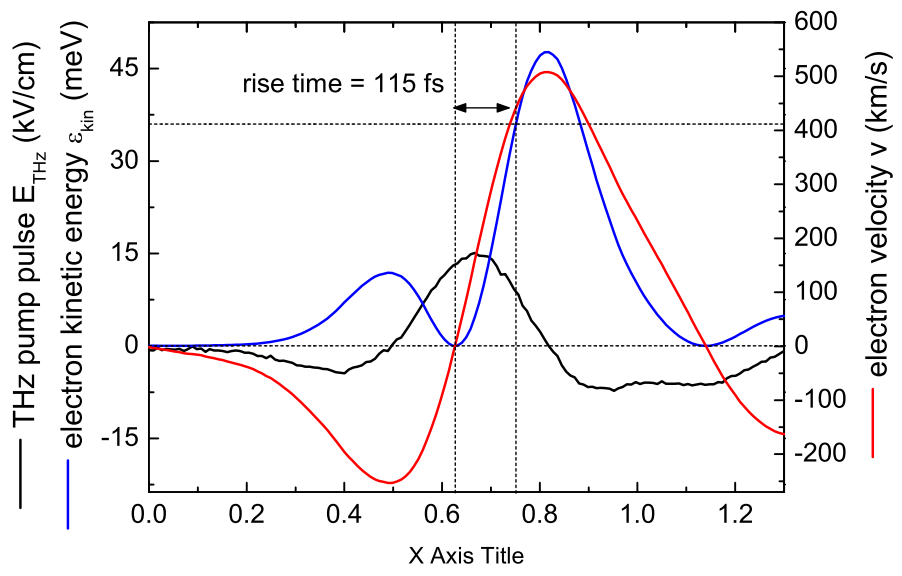

Figure 6.6: $\mathrm{THz}$ pump pulse (black solid line), electron velocity (red solid line) and the corresponding kinetic energy of the electron (blue solid line). The energy of the $\mathrm{LO}$ phonon of $36 \mathrm{meV}$ is indicated in the dashed line. The rise time of the kinetic energy is marked.

The maximum field of only $15 \mathrm{kV} / \mathrm{cm}$ already accelerates the electron to kinetic energies of more than $45 \mathrm{meV}$. The emission threshold for LO phonons of $36 \mathrm{meV}$ is reached in approximately $100 \mathrm{fs}$, which is shorter than the LO phonon oscillation period. Hence, the electron experiences a displacement along the internal coordinate $r$, which is shown in Figure 6.5 (e) and (f). Owing to the impulsive character of the energy transfer, coherent LO phonon oscillations appear as a stern wave of the moving electron, similar to wakefields in plasmas $[197,198]$. With increasing strength of such oscillations, the resulting electric field from the lattice polarization alters the motion of the electron, leading to electron oscillations along the internal coordinate $\mathrm{r}$ with the frequency of the lattice vibration $\omega_{L O}$. The electron oscillations correspond to a periodic modulation of the momentary electron velocity $\mathrm{v}_{e}(\mathrm{t})$ on top of the saturation drift velocity $\mathrm{v}_{0}(\mathrm{t})$ of the entire quasiparticle:

$$
v_{e}(t)=\frac{d r}{d t}+v_{0}
$$

The electron explores velocity regions that are characterized by different differential mobilities with positive or negative sign, depending on the relative phase between the lattice polarization and the external electric field. The 


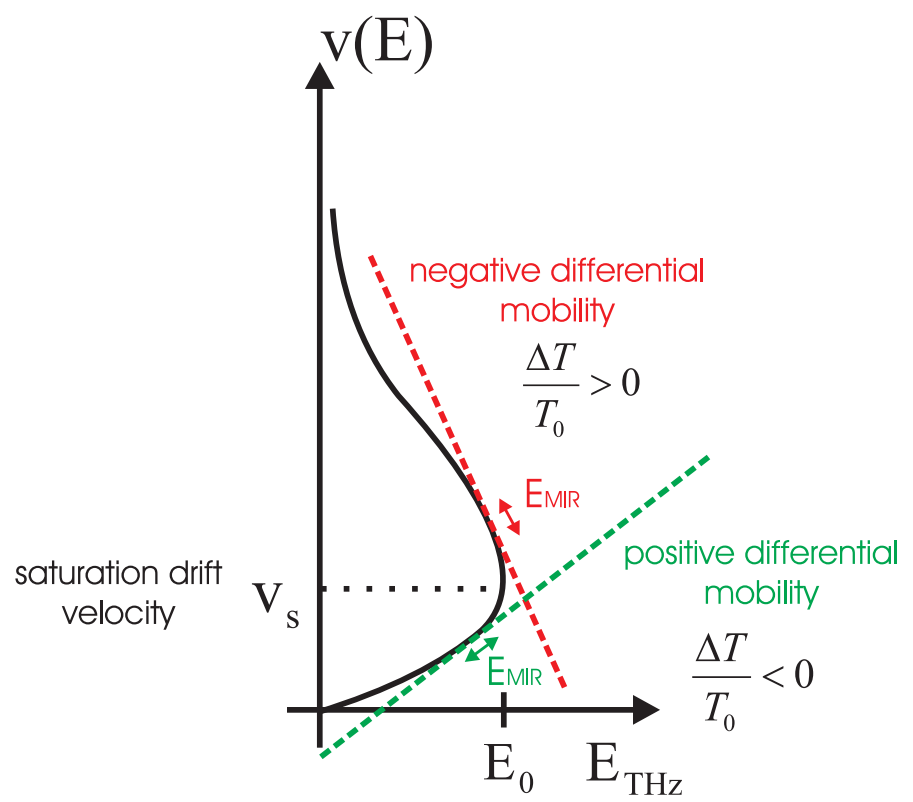

Figure 6.7: Electron velocity plotted over the electric field strength. The saturation velocity $\mathrm{v}_{s}$ due to the breakup of the polaron is marked. For lower electric

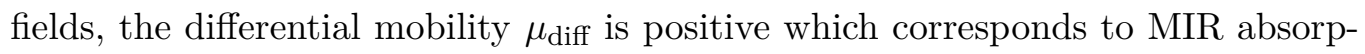
tion. Above the saturation velocity $\mu_{\text {diff }}$ is negative corresponding to MIR gain. The external $\mathrm{THz}$ field $\mathrm{E}_{0}$ accelerates the electron to the saturation drift velocity $\mathrm{v}_{s}$.

differential mobility is given by

$$
\mu_{\mathrm{diff}}\left(v_{e}\right)=\left(\frac{\partial E}{\partial v_{e}}\right)^{-1}
$$

The instantaneous differential mobility can be deduced from the electric field dependence of the velocity $\mathrm{v}_{e}(\mathrm{E})$ as depicted in Figure 6.7.

For a positive slope of $\partial E / \partial v_{e}$ a high frequency electric field induces a current component of the same phase resulting in mid-infrared absorption. In Figure 6.7 this situation is depicted by the green tangent to the fieldvelocity curve. For $\partial E / \partial v_{e}<0$ the induced current has the opposite phase, thus amplifying the mid-infrared radiation. This is shown by the red tangent to the field-velocity curve in Figure 6.8. The saturation drift velocity $\mathrm{v}_{s}$ is reached at an external $\mathrm{THz}$ field strength $\mathrm{E}_{0}$.

In the following, probing of the differential mobility by measuring the mid-infrared transmission as shown in Figure 6.7, is explained: an electron is considered to be exposed to both a strong DC electric field represented by the $\mathrm{THz}$ pulse and a weak AC field, which corresponds to the MIR probe 
pulse. The equation of motion reads

$$
m_{\mathrm{eff}} \frac{d v_{e}}{d t}=-F\left(v_{e}\right)+e E_{\mathrm{THz}}+e E_{\mathrm{MIR}}
$$

Here, $F\left(v_{e}\right)$ is a velocity dependent friction force due to electron LO-phonon interaction as discussed in Figure 6.5. Assuming quasi-stationary transport, the electron velocity is composed of a stationary velocity component $v_{D C}$ due to the electric field of the $\mathrm{THz}$ pulse and an oscillating velocity component $v_{A C}$ due to the MIR probe pulse. For weak MIR probe fields, equation (6.5) can be expanded around the working point $v_{D C}$. Truncation of the expansion after the first order results in

$$
m_{\mathrm{eff}} \frac{d v_{\mathrm{AC}}}{d t} \approx\left[e E_{\mathrm{DC}}-F\left(v_{\mathrm{DC}}\right)\right]+e E_{\mathrm{AC}}-m_{\mathrm{eff}} v_{\mathrm{AC}} \cdot\left[\frac{\partial F(v)}{\partial v}\right]_{v=v_{\mathrm{DC}}}
$$

Under quasi-stationary conditions the electric force of the DC field equals the friction of the lattice. Hence, $\left[e E_{\mathrm{DC}}-F\left(v_{\mathrm{DC}}\right)\right]=0$. With the relation $v=\mu E$ and with equation (6.6), a velocity and probe frequency dependent mobility of the electron is defined

$$
\mu_{\mathrm{AC}}\left(v_{e}, \omega\right)=\frac{1}{i \omega m_{\mathrm{eff}} / e+m_{\mathrm{eff}} / e \cdot\left(\partial F / \partial v_{e}\right)}=\frac{1}{i \omega m_{\mathrm{eff}} / e+\left[\mu_{\mathrm{diff}}\left(v_{e}\right)\right]^{-1}} .
$$

The real part of equation (6.7) determines the mid-infrared gain and absorption.

$$
\frac{\Delta T\left(v_{e}, \omega\right)}{T_{0}} \propto \operatorname{Re}\left[\mu_{\mathrm{AC}}\left(v_{e}, \omega\right)\right]
$$

This simple model shows, that the mid-infrared transmission is an effective probe for the instantaneous electron velocity. However, it does not fully describe the experimentally observed MIR transmission change. In particular, the assumption of a quasi-stationary transport is certainly incorrect on ultrafast time scales. The observed phenomena rather exhibit strong memory effects which are neglected here. The full model discussed in Section 6.2.2 correctly accounts for such non-markovian behavior.

The experimentally observed $\mathrm{THz}$ induced modulation of the mid-infrared transmission corresponds to a periodic shift of the working point $v_{D C}$ around the saturation drift velocity $\mathrm{v}_{s}$. The electron velocity oscillates between positive and negative differential mobility and the mid-infrared pulse experiences absorption or gain, respectively. The observed oscillation frequency is the frequency of the LO phonon $\omega_{L O}$. This is shown in Figure 6.8. 


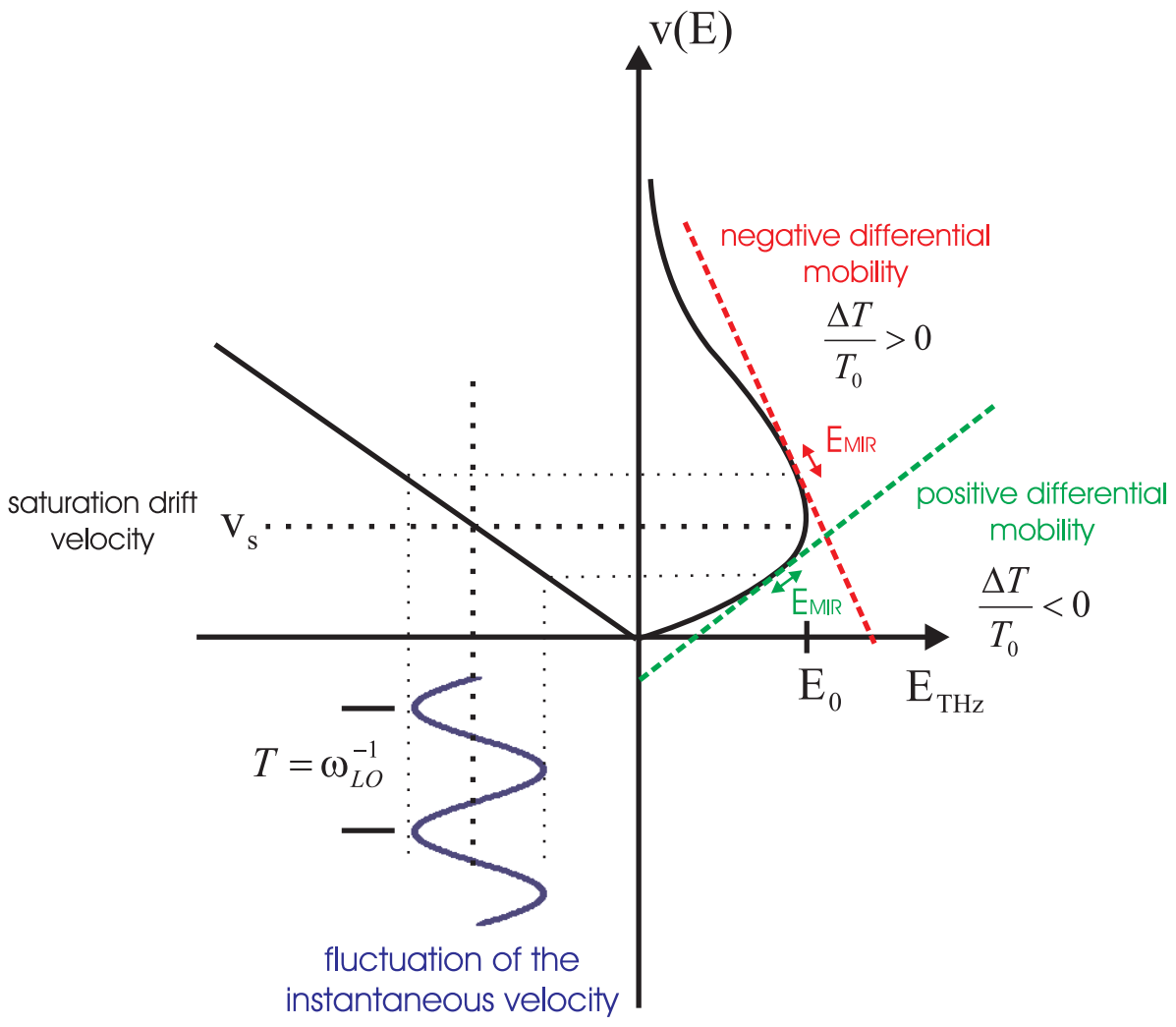

Figure 6.8: The impulsive acceleration of the electron triggers coherent lattice vibrations with the frequency of the LO phonon $\omega_{L O}$. These oscillations shift the quasi-stationary DC velocity around the saturation drift velocity $\mathrm{v}_{s}$ such that the MIR probe pulse experiences either positive or negative differential electron mobilities.

The assumption of an instantaneous mobility underlaying the phenomenological picture derived in Figure 6.8 is a serious approximation. An accurate description in the quantum kinetic regime must account for the finite duration of scattering events which lead to the friction force $F$ on the electron. A full theoretical treatment of the observed effect is presented in the following paragraph.

\subsubsection{Quantum kinetic description of the internal po- laron dynamics}

In order to explain the experimentally observed nonlinear polaron dynamics, a theoretical model was derived. It is based on the solution of the Heisenberg equation of motion of the coupled electron-phonon system driven by the $\mathrm{THz}$ 
pump and MIR probe radiation. A similar approach was already presented in reference [199].

The model considers a single electron interacting with the local electric field and the LO phonon modes of the crystal via polar optical mode scattering. The ensemble response is obtained by multiplication with the electron density. The local electric field $\mathrm{E}_{\mathrm{loc}}(\mathrm{t})$ is the sum of the applied $\mathrm{THz}$ and MIR fields plus the field re-emitted from the coherent motions of the electrons in the sample. It contains the linear and the nonlinear response of the system and accounts correctly for the radiative damping of the electron motion [179]:

$$
E_{\mathrm{loc}}(t)=E_{\mathrm{THz}}(t)+E_{\mathrm{MIR}}(t)-\frac{1}{2} e N_{e}\langle v(t)\rangle Z_{0} d .
$$

$e, \mathrm{~N}_{e}, \mathrm{Z}_{0}$ and $d$ are the electron charge, the volume density of electrons, the vacuum impedance and the layer thickness, respectively. The linear and nonlinear response of the coupled electron-phonon system is contained in the time dependent statistical average velocity, i.e., the expectation value of the velocity operator. It can be gained from the Heisenberg equation of motion:

$$
\langle v(t)\rangle=\left\langle\frac{d x}{d t}\right\rangle=\frac{i}{\hbar}\langle[H, x(t)]\rangle,
$$

where $x$ is the direction of the electric field and the quantum mechanical Hamiltonian is given by[200]

$$
\begin{aligned}
H(t)= & \frac{\vec{p}^{2}}{2 m_{\mathrm{eff}}}+e x E_{l o c}(t)+\sum_{\vec{q}} \frac{P_{\vec{q}}^{2}+\omega_{L O}^{2} Q_{\vec{q}}^{2}}{2} \\
& +\sqrt{\frac{e^{2}}{V \epsilon_{0}}\left(\frac{1}{\epsilon_{\infty}}-\frac{1}{\epsilon_{s}}\right)} \times \sum_{\vec{q}} \frac{P_{\vec{q}} \cos (\vec{q} \cdot \vec{r})+\omega_{L O} Q_{\vec{q}} \sin (\vec{q} \cdot \vec{r})}{|\vec{q}|} .
\end{aligned}
$$

Equation (6.11) assumes a collective motion of the electron ensemble in the external electric field. The spatial coordinate $r$ accounts for the center of mass motion of the ensemble. Therefore, equation (6.11) does not include an interaction term for electron-electron scattering, which depends on the relative spatial coordinate. Equation (6.11) is the reduced one-electron Hamiltonian from equation (2.15). In order to solve the Heisenberg equation of motion (6.10) one has to know the time-dependent expectation value of the commutator, which yields another similar equation for the velocity operator. Solving for the commutator in the equation for the velocity operator yields in turn new equations for the phonon coordinates $\mathrm{Q}_{\vec{q}}$ and $\mathrm{P}_{\vec{q}}$. In fact, equation (6.10) is the starting point of an infinite hierarchy of equations which 
have to be solved simultaneously. The infinite set of coupled equations is closed by establishing the equations of motion for the operators $x, p, Q_{\vec{q}}$ and $P_{\vec{q}}$ and subsequent Taylor expansion to the second order. Note that due to the theorem of Ehrenfest [20] the Heisenberg equations of motion for expectation values of quantum mechanical harmonic oscillators are identical to the classical counterparts in Hamilton mechanics. Therefore the second order expansion corresponds to a first order quantum mechanical correction of the classical equation of motion. At this point it should be remembered, that the classical lattice restoration force introduced in Section 2.3.3 accurately describes the electron propagation in a polar lattice above the phonon resonance. The quantum mechanical correction extends the validity range of the classical model to lower frequencies. The correction term corresponds to a finite elongation in real space of the electron wavepacket. The truncation of the Taylor expansion corresponds to the assumption that the electron size is constant during the measurement process. Both conditions are expressed in the finite and constant polaron radius $\mathrm{R}$ given in equation (2.38).

\section{Derivation of the Heisenberg equation of motion}

In the following the equation of motion for the operators $x, p, Q_{\vec{q}}$ and $P_{\vec{q}}$ are listed. A detailed derivation can be found in Appendix E.

$$
\begin{aligned}
\frac{d x}{d t}= & \frac{i}{\hbar}[H, x]=\frac{p}{m}=v \\
\frac{d p}{d t}= & \frac{i}{\hbar}[H, p]=-e E_{\mathrm{loc}}(t) \\
& +\sqrt{\frac{e^{2}}{V \epsilon_{0}}\left(\frac{1}{\epsilon_{\infty}}-\frac{1}{\epsilon_{s}}\right)} \times \sum_{\vec{q}} \frac{P_{\vec{q}} q_{x} \sin (\vec{q} \cdot \vec{r})-\omega_{L O} Q_{\vec{q}} q_{x} \sin (\vec{q} \cdot \vec{r})}{|\vec{q}|} \\
\frac{d Q_{\vec{q}}}{d t}= & \frac{i}{\hbar}\left[H, Q_{\vec{q}}\right]=P_{\vec{q}}+\sqrt{\frac{e^{2}}{V \epsilon_{0}}\left(\frac{1}{\epsilon_{\infty}}-\frac{1}{\epsilon_{s}}\right)} \frac{\cos (\vec{q} \cdot \vec{r})}{|\vec{q}|} \\
\frac{d P_{\vec{q}}}{d t}= & \frac{i}{\hbar}\left[H, P_{\vec{q}}\right]=-\omega_{L O}^{2} Q_{\vec{q}}-\sqrt{\frac{e^{2}}{V \epsilon_{0}}\left(\frac{1}{\epsilon_{\infty}}-\frac{1}{\epsilon_{s}}\right)} \frac{\omega_{L O} \sin (\vec{q} \cdot \vec{r})}{|\vec{q}|}
\end{aligned}
$$

The next step is to derive the equations of motion for the operators appearing on the right hand side in equations (6.12), i.e., for $\mathrm{P}_{\vec{q}} \sin (\vec{q} \cdot \vec{r}), \mathrm{Q}_{\vec{q}} \cos (\vec{q}$. $\vec{r}), \sin (\vec{q} \cdot \vec{r})$ and $\cos (\vec{q} \cdot \vec{r})$. In order to close the infinite set of equations the operators are expanded and subsequently approximated. One thereby obtains the expectation values of the relevant observables listed above. 


\section{Expansion and approximation of the Heisenberg operators}

The infinite expansion series of the three and four dimensional operators is truncated at the second order. The resulting expressions are simplified by making use of cylindrical symmetry for the case of a propagating electron, i.e., with an applied external electric field, or of spherical symmetry in equilibrium, i.e., without incident electric field. In a last step the resulting expression is approximated by an exponential function. A detailed description of the operator expansion and approximation is given in Appendix F.

The resulting expressions for the quantum mechanical operators are:

$$
\begin{aligned}
\left\langle P_{\vec{q}} \sin (\vec{q} \cdot \vec{r})\right\rangle & =\left\langle P_{\vec{q}}\right\rangle \sin \left(q_{x}\langle x\rangle\right) \times \exp \left[-\frac{\vec{q}^{2}}{2} R^{2}\right], \\
\left\langle Q_{\vec{q}} \cos (\vec{q} \cdot \vec{r})\right\rangle & =\left\langle Q_{\vec{q}}\right\rangle \cos \left(q_{x}\langle x\rangle\right) \times \exp \left[-\frac{\vec{q}^{2}}{2} R^{2}\right], \\
\langle\cos (\vec{q} \cdot \vec{r})\rangle & =\cos \left(q_{x}\langle x\rangle\right) \times \exp \left[-\frac{\vec{q}^{2}}{2} R^{2}\right], \\
\langle\sin (\vec{q} \cdot \vec{r})\rangle & =\sin \left(q_{x}\langle x\rangle\right) \times \exp \left[-\frac{\vec{q}^{2}}{2} R^{2}\right],
\end{aligned}
$$

where $x$ is the direction of the electron propagation. The exponential term in the quantum mechanical operator determines the size of the electron wavepacket in the self-consistent polaron potential. The size of the wavepacket determines the coupling to the LO phonons in momentum space via the resonance condition $\omega=\vec{q} \cdot \vec{v}$. The electron couples to a LO phonon only if

$$
R<<1 / \vec{q}
$$

i.e., if the wavepacket in real space is much smaller than the wavelength of the LO phonon. Note that the Fröhlich interaction prefers coupling to LO phonons with small wavevectors due to the $\mathrm{q}^{-2}$-dependence of the scattering matrix element. The coupling condition is described in Figure 6.9. If the phonon wavelength is long compared to the size of the electron wavepacket, the electron experiences a spatially varying potential. For phonons with short wavelengths, i.e., high wavevectors $\vec{q}$, the electron experiences a spatially homogeneous average potential.

The reason why the classical model described in Section 2.3.3 fails, is that in the classical limit the electron is assumed to be a point-like charged particle. Therefore it still couples to phonons with the shortest wavelengths or the highest $\vec{q}$-wavevectors. The quantum mechanical correction, i.e., the exponential terms derived in the expressions (6.13) limits the coupling in the momentum space. It therefore already modifies the linear response, i.e., 


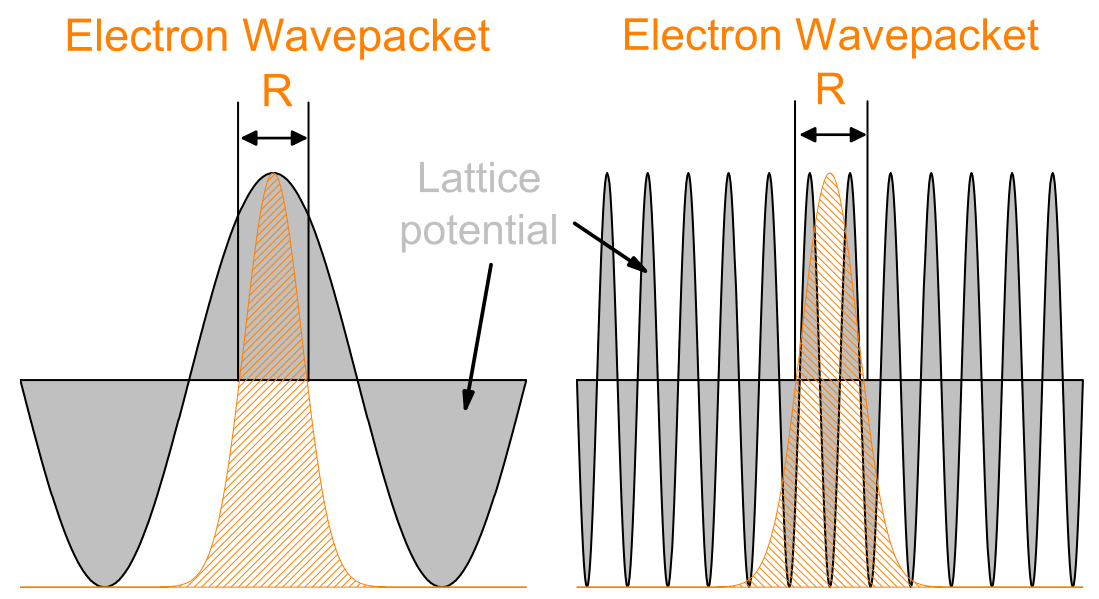

Figure 6.9: Coupling of the lattice potential to the electron. For long wavelength phonon oscillations (left side) compared to the wavepacket size $R$, the electron sees a spatially dependent potential. For short wavelength phonons (right side), the electron sees only an average potential.

the self-consistent polaron potential and the energy exchange between the electrons and the lattice after acceleration by a moderate electric field. In order to obtain the final form of the equations of motion of the quantum mechanical operators, as they were implemented in the theoretical model, one must combine equations (6.12) with the set of equations (6.13):

$$
\begin{aligned}
\frac{d\langle x\rangle}{d t}= & \frac{\langle p\rangle}{m}=\langle v\rangle, \\
\frac{d\left\langle p_{x}\right\rangle}{d t}= & e E_{l o c}(t)+\sqrt{\frac{e^{2}}{V \epsilon_{0}}\left(\frac{1}{\epsilon_{\infty}}-\frac{1}{\epsilon_{s}}\right)}, \\
& \times \sum_{\vec{q}} \frac{P_{\vec{q}} q_{x} \sin \left(q_{x}\langle x\rangle\right)-Q_{\vec{q}} q_{x} \omega_{L O} \cos \left(q_{x}\langle x\rangle\right)}{|\vec{q}|} \exp \left[-\frac{\vec{q}^{2}}{2} R^{2}\right], \\
\frac{d\left\langle Q_{\vec{q}}\right\rangle}{d t}= & \left\langle P_{\vec{q}}\right\rangle+\sqrt{\frac{e^{2}}{V \epsilon_{0}}\left(\frac{1}{\epsilon_{\infty}}-\frac{1}{\epsilon_{s}}\right)} \times \frac{\cos \left(q_{x}\langle x\rangle\right)}{|\vec{q}|} \exp \left[-\frac{\vec{q}^{2}}{2} R^{2}\right], \\
\frac{d\left\langle Q_{\vec{q}}\right\rangle}{d t}= & -\omega_{L O}^{2}\left\langle Q_{\vec{q}}\right\rangle-\sqrt{\frac{e^{2}}{V \epsilon_{0}}\left(\frac{1}{\epsilon_{\infty}}-\frac{1}{\epsilon_{s}}\right)} \times \frac{\omega_{L O} \sin \left(q_{x}\langle x\rangle\right)}{|\vec{q}|} \exp \left[-\frac{\vec{q}^{2}}{2} R^{2}\right] .
\end{aligned}
$$

The finite, constant size of the electron wavepacket results from the truncation of the expansion of the operators after the second order. This approximation yields good results on short time scales. On longer time scales, the 


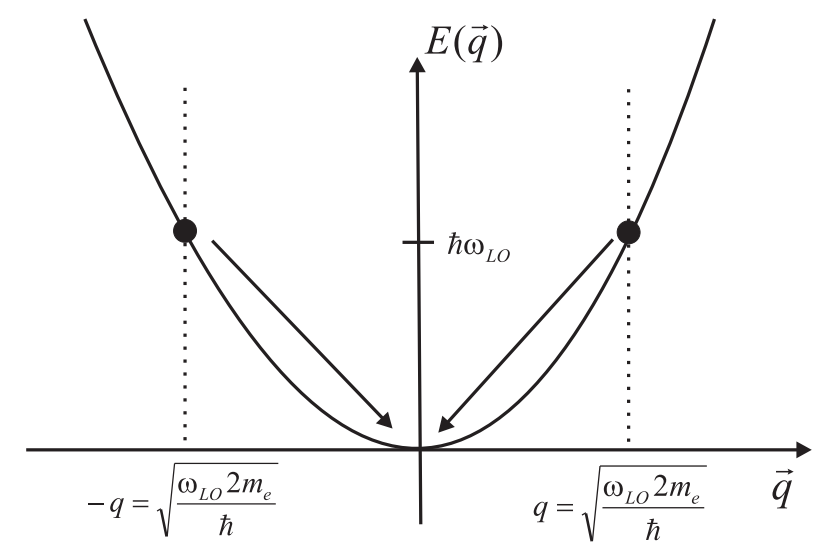

Figure 6.10: Reduced momentum space with only two phonon modes allowed. Both modes provide energy and momentum conservation during scattering with an electron which relaxes to the conduction band minimum.

evolution of the electron wavepacket must be taken into account, i.e., the truncation after the second order is insufficient for a correct description of the electron propagation. Therefore, the model calculations presented in the next paragraph solve equations (6.15) only in a short time interval, where the wavepacket size is conserved after the ultrafast acceleration of the electron by the THz field. Another parameter having a high influence on the accuracy of the model is the number of phonon modes included in the calculation. While the model already yields good results when only two phonon modes are taken into account, a greater number must be chosen in order to properly compare the calculation with the experiment. In the model calculations presented in the following section, the number of phonon modes was set to 300. In that case the calculation on a conventional personal computer takes approximately 24 hours. Therefore less accurate calculations were performed, where only two phonon modes were included. Such calculations take approximately 5 minutes and allow to test different parameter settings of the model.

\subsubsection{Model calculations}

In the model calculation, equations (6.15) were numerically integrated with the Runge-Kutta method [201]. The input parameters are the $\mathrm{THz}$ and the MIR pulse and the model solves for the local electric field as defined in equation (6.9). The analysis of the simulation result is done exactly in the same way as the analysis of the measured data, i.e., by solving for the change in the MIR transition via equation (3.24).

At the beginning model calculations were performed in a simplified electron- 

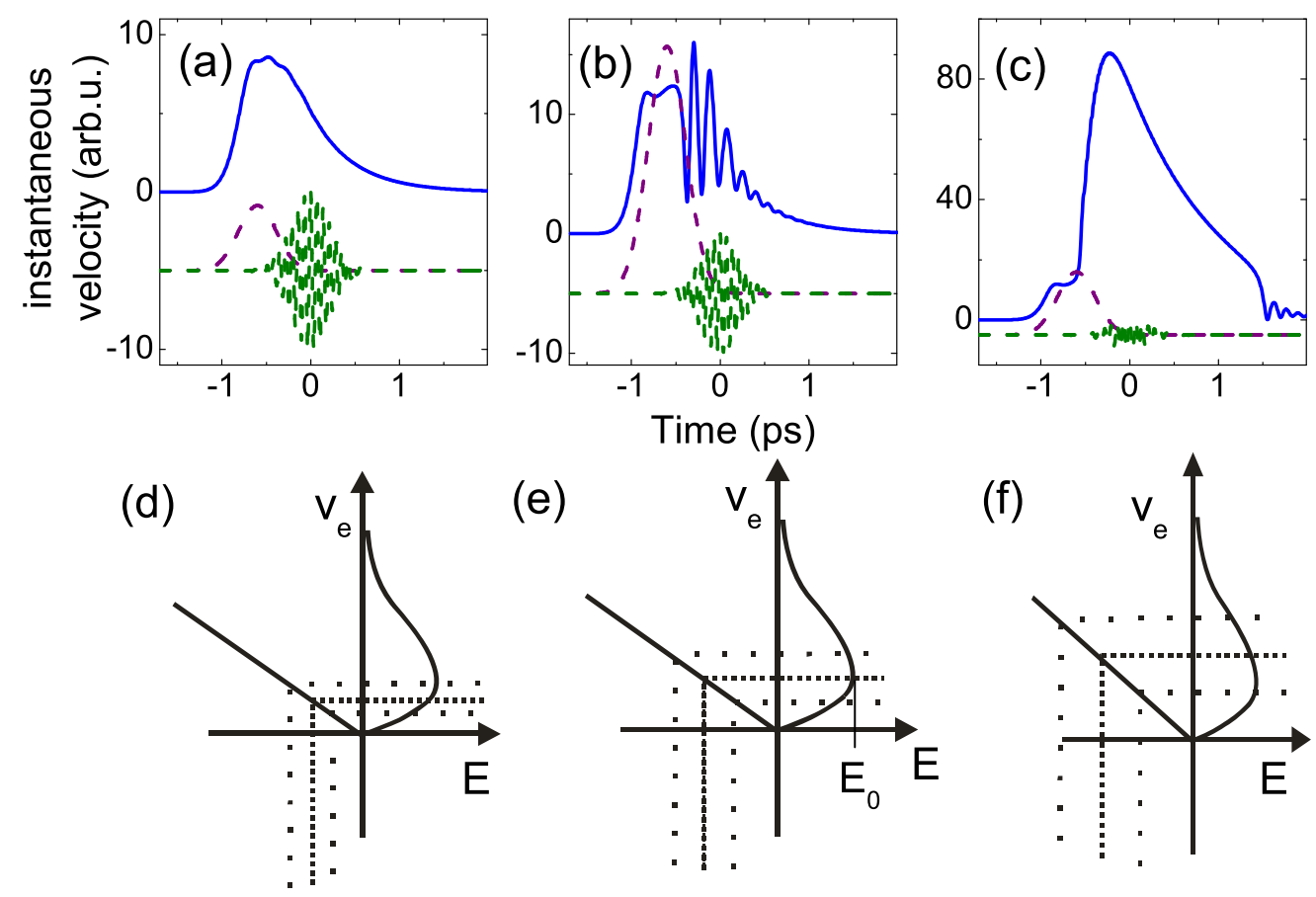

Figure 6.11: Instantaneous electron velocity $\mathrm{v}_{e}(\mathrm{t})$ (blue solid line) under influence of a THz pump pulse (purple dashed line) with $0.2 \cdot \mathrm{E}_{0}(\mathrm{a}), \cdot \mathrm{E}_{0}(\mathrm{~b})$ and $1.01 \cdot \mathrm{E}_{0}$ (c) intensity and a MIR probe pulse (green dashed line). (d)-(f) Corresponding quasistationary regime.

LO phonon system, where only two LO-phonon modes were allowed. The modes are selected by the resonance condition

$$
\hbar \omega_{L O}=\frac{\hbar^{2} \vec{q}^{2}}{2 m_{\mathrm{eff}}}
$$

which yields the phonon wavevectors

$$
\mp \vec{q}=\sqrt{\frac{\omega_{L O} 2 m_{\mathrm{eff}}}{\hbar} .}
$$

The system with two allowed phonon modes is depicted in momentum space in Figure 6.10.

Figure 6.11 (a)-(c) shows the resulting time dependent quasi-stationary velocity $\mathrm{v}_{e}(\mathrm{t})$ (blue solid line) for an incident $\mathrm{THz}$ pulse (purple dashed line) 


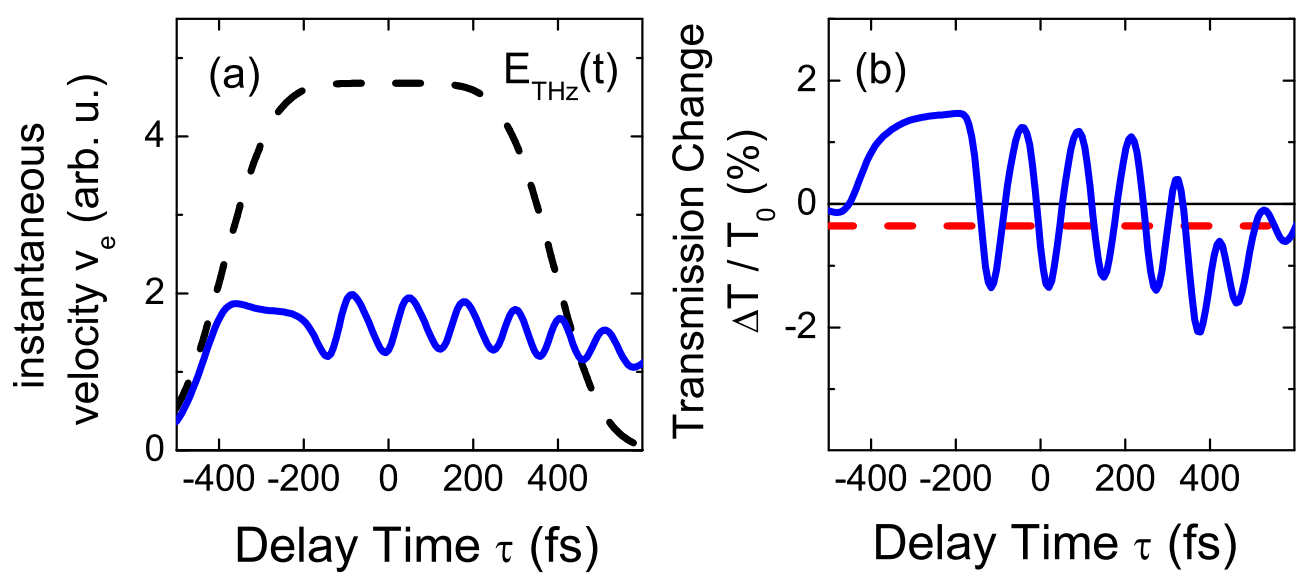

Figure 6.12: (a) Instantaneous electron velocity $\mathrm{v}_{e}(\mathrm{t})$ (blue solid line) after excitation with a THz pump pulse (black dashed line). (b) Transmission change over the THz pump - MIR probe delay time $\tau$ (blue solid line). For comparison, the linear interband absorption is shown (red dashed line).

with different relative electric field amplitudes and for an incident MIR probe pulse (green dashed line) with fixed intensity. Figure 6.11 (d)-(f) show the corresponding quasi-stationary regime.

In Figure 6.11 (a), the velocity increases almost linearly during the presence of the weak $\mathrm{THz}$ field. Only around the maximum velocity, slight oscillations of the velocity set in. The amplitude of the $\mathrm{THz}$ pulse is $20 \%$ of the saturation field $E_{0}$. In Figure 6.11 (b) one observes a saturation of the velocity for approximately 600 fs before strong velocity oscillations occur. Here, the $\mathrm{THz}$ field strength is $\mathrm{E}_{0}$, i.e., the electron is accelerated to the saturation drift velocity $\mathrm{v}_{s}$ on a time scale shorter than the lattice response time. Hence, strong velocity oscillations appear, which result from acceleration and deceleration of the electron in the coherent phonon potential. By increasing the $\mathrm{THz}$ field by $1 \%$, the electron is completely detached from the surrounding phonon cloud and accelerated to extremely high velocities. This situation is shown in Figure 6.11 (c). After the $\mathrm{THz}$ pulse one observes a monotonous deceleration.

The interpretation of such a behavior is shown in the corresponding quasistationary regime plotted in Figure 6.11 (d)-(f). For low $\mathrm{THz}$ fields, the displacement of the electron from the polaron center is rather low and the saturation velocity $\mathrm{v}_{s}$ is not reached. At a THz field strength $\mathrm{E}_{0}$ the operating 


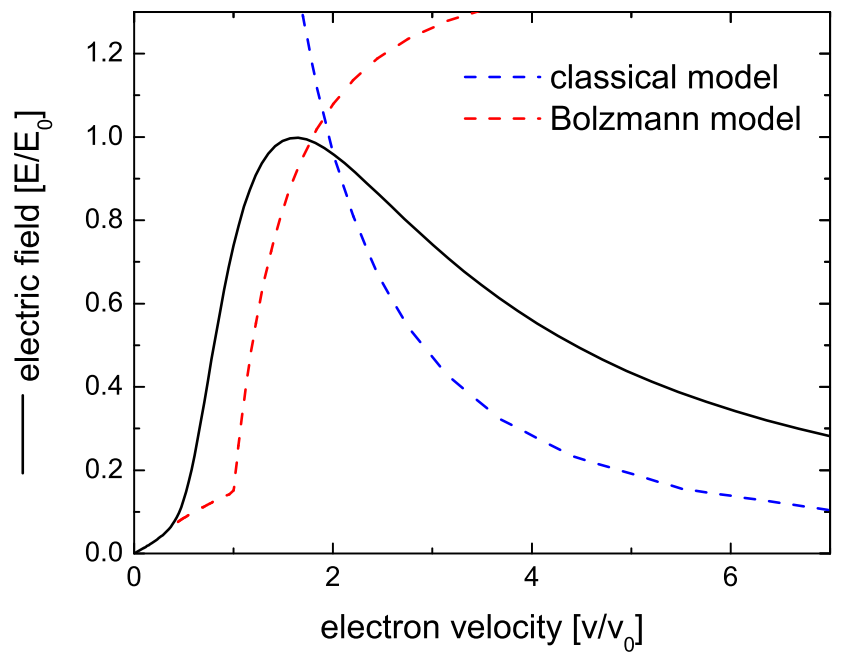

Figure 6.13: Electric field dependence of the electron velocity calculated with the full model (black solid line). The friction force after Fermi's Golden Rule as assumed in the Boltzmann transport theory (red dashed line) and after the classical model as discussed in Section 2.3.3 (blue dashed line) are shown for comparison.

point is fixed at the saturation velocity and the electron performs maximal oscillations in the polaron potential. At an even higher field strength, the electron is undressed from the virtual phonon cloud and can therefore attain a significantly higher velocity. The undressing of the electron requires only a very slight increase of the $\mathrm{THz}$ field of only $1 \%$, which is due to the very limited number of phonon modes included in the simulation. The oscillation frequency of $\mathrm{v}_{e}(\mathrm{t})$ in Figure 6.11 (a)-(c) is not constant and also lower than the LO phonon frequency, which also results from the limited number of phonon modes. A simulation including 300 phonon modes is shown in Figure 6.12.

One observes a rise of the instantaneous velocity comparable to Figure 6.11 (b). The resulting oscillation frequency is constant and the calculated transmission change in Figure 6.12 (b) shows an oscillation at the frequency of the LO phonon. The result of the full calculation reproduces the measured effect of the modulation of the MIR transmission with the frequency of the LO phonon.

The calculated electric field dependent electron velocity is plotted in Figure 6.13. The velocity is normalized to the threshold velocity $\mathrm{v}_{0}$, which cor- 
responds to a kinetic electron energy of the LO phonon: $v_{0}=\sqrt{2 \hbar \omega_{L O} / m_{\text {eff }}}$. The saturation drift velocity resulting from the model calculation is $1.7 \cdot \mathrm{v}_{0}$. Assuming an acceleration time of $t_{\text {acc }}=100 \mathrm{fs}$, one finds for the saturation field $\mathrm{E}_{0}$

$$
E_{0}=\frac{\sqrt{2 \hbar \omega_{L O} m_{\mathrm{eff}}}}{e t_{\mathrm{acc}}} \approx 27.8 \frac{\mathrm{kV}}{\mathrm{cm}} .
$$

The electric fields applied in the experiment should therefore trigger strong lattice oscillations, without completely removing the electron from the polaron center.

It is instructive to compare the result of the model calculation (black solid line) with the friction force after Fermi's Golden Rule as assumed in the Boltzmann transport theory (red dashed line) and after the classical model (blue dashed line) as discussed in Section 2.3.3. Below and above the saturation drift velocity, the simulated field-velocity curve reproduces qualitatively the results of the Boltzmann and the classical model, respectively. In addition, the quantum kinetic model derived here correctly reproduces the field dependence of the electron near the saturation drift velocity aa observed in the experiment. 


\section{Chapter 7}

\section{Summary}

In this thesis the ultrafast dynamics of electrons in a polar semiconductor are studied in intense electric fields. This subject is highly relevant for technological applications in modern electronics, since the miniaturization of devices leads to stronger internal fields and higher operation frequencies. In addition, the coherent manipulation of electrons as shown in this thesis can be exploited for coherent control and quantum information processing.

The experiments presented here use ultrashort $\mathrm{THz}$ and mid-infrared pulses for excitation and subsequent probing, respectively. The radiation is detected in amplitude and phase by electro-optic sampling, which yields the full information on the electric field. Single-cycle $\mathrm{THz}$ pulses with field amplitudes of up to $400 \mathrm{kV} / \mathrm{cm}$ were generated via four-wave rectification in optically ionized dry nitrogen gas. Ultrashort mid-infrared pulses were generated in a GaSe crystal via phase-matched type-I difference frequency mixing. In this thesis, the first THz-pump mid-infrared-probe setup was developed, which allows a time-resolved detection of the individual transients.

Our novel experiments gave insights into the underlying physics of the following nonlinear $\mathrm{THz}$-phenomena:

- Phase-resolved nonlinear propagation experiments on n-type gallium arsenide with ultrashort, intense $\mathrm{THz}$ pulses reveal a coherent emission at $2 \mathrm{THz}$ with picosecond decay times. While the linear $\mathrm{THz}$ response is in perfect agreement with the Drude response of free electrons, the nonlinear response is dominated by the super-radiant decay of inverted impurity transitions. Ultrafast electron redistribution in higher lying impurity states and in the conduction band is essential for establishing a population inversion in impurity atoms with unpopulated ground state. 
A quantum mechanical discrete state model using the potential of the disordered impurities accounts for the experimental observations.

- The nonlinear optical response of shallow donor transitions in n-type GaAs is studied in ultrafast phase-resolved $\mathrm{THz}$ propagation experiments. At a lattice temperature of $100 \mathrm{~K}$, carrier-wave Rabi oscillations are observed at $\mathrm{THz}$ field strength of up to $5 \mathrm{kV} / \mathrm{cm}$. Strong radiative coupling between impurity transitions results in a coherently excited macroscopic polarization which oscillates for several picoseconds. The observed oscillations are reproduced by model calculations using the coupled Maxwell-Bloch equations for an ensemble of radiatively coupled two-level systems. A comparison with the simulation shows that the two-level approach breaks down for driving fields above $5 \mathrm{kV} / \mathrm{cm}$. Absorption measurements of shallow impurities show that the linear response at higher doping concentrations is governed by many-body interactions which are still not fully understood.

- For the first time, the internal degree of freedom of the Fröhlich polaron is directly measured in the nonlinear quantum kinetic transport regime. Ultrafast acceleration of conduction band electrons in the electric field of a $\mathrm{THz}$ pump pulse triggers coherent lattice vibrations which lead to electron oscillations along the internal coordinate in the self-consistent polaron potential. The concomitant drift velocity oscillations modulate the optical response at mid-infrared frequencies between absorption and stimulated emission.

In this work, time-resolved ultrafast nonlinear THz spectroscopy is demonstrated for the first time. This new spectroscopic tool, which relies on $\mathrm{THz}$ pulses combining high electric field amplitudes with high time-resolution, can be applied to study the $\mathrm{THz}$ response in a variety of systems. Examples are atomic or molecular systems in intense electric fields, the nonlinear field-induced generation of electrons from surfaces or field-induced control of chemical reactions. Electrons bound to donor atoms may be used as potential Qbits in future quantum computers. The large dipole moment of the $1 \mathrm{~S} \rightarrow 2 \mathrm{P}$ transition and the high dielectric constant make n-doped gallium arsenide a candidate for coherent quantum control experiments. The direct monitoring of free carrier transport in a polar lattice allows for a detailed investigation of quantum kinetic processes in semiconductors. The experiments can be optimized through an independent tailoring of the THz pump and mid-infrared probe pulses. This can be achieved either by spectral shaping of the optical pulse used for $\mathrm{THz}$ generation or by directly shaping the $\mathrm{THz}$ pulse. 
Nonlinear electron transport in highly polar materials, like e.g., II-VI semiconductor compounds, should yield an even more pronounced modulation of the mid-infrared transmission [202]. 


\section{Appendix A}

\section{List of abbreviations}

$\begin{array}{ll}\mathrm{m}_{e} & \text { free electron mass } \\ c & \text { vacuum speed of light } \\ e & \text { elementary charge of the electron } \\ h & \text { Planck's constant } \\ \hbar=\mathrm{h} / 2 \pi & \text { normalized Planck's constant } \\ \mathrm{m}_{\mathrm{eff}} & \text { effective electron mass } \\ \mathrm{a}_{l} & \text { lattice constant } \\ \mathrm{k}_{B} & \text { Boltzmann factor } \\ \mathrm{E}_{F} & \text { Fermi energy } \\ \mathrm{E}_{C} & \text { Conduction band offset energy } \\ \mathrm{T} & \text { Temperature } \\ \epsilon_{s} & \text { static background dielectric constant } \\ \epsilon_{\infty} & \text { high frequency dielectric constant } \\ \mathrm{R}_{y} & \text { Rydberg constant } \\ \mathrm{a}_{B} & \text { effective Bohr radius } \\ \vec{d} & \text { electric dipole operator } \\ \varrho & \text { density matrix } \\ \Omega & \text { Rabi frequency } \\ \mu & \text { electron mobility } \\ \sigma & \text { electric conductivity } \\ \alpha e p & \text { electron-phonon coupling constant } \\ \alpha & \text { absorption coefficient } \\ \sigma & \text { conductivity } \\ \mathrm{T}_{1} & \text { population relaxation time } \\ \mathrm{T}_{2} & \text { dephasing time } \\ & \end{array}$




\section{Appendix B}

\section{Charge transport and optical absorption}

In this appendix, the relation between typical transport parameters such as the frequency dependent mobility $\mu(\omega)$ and optical parameters such as the absorption coefficient $\alpha(\omega)$ is given. A more detailed derivation is found in [64].

The conductivity of a doped semiconductor follows from the electron mobility as given by equations (2.21) and (2.22). The medium response to an external electric field is described by the dielectric constant [203]

$$
\epsilon(\omega)=\epsilon_{b}+i \frac{\sigma(\omega)}{\omega}=\epsilon^{\prime}+i \epsilon^{\prime \prime}
$$

where $\epsilon^{\prime}$ and $\epsilon^{\prime \prime}$ are the real and imaginary part of the dielectric function. For optical frequencies, the material is usually described by a frequency dependent refractive index $\mathrm{n}(\omega)$, which is related to the dielectric function by

$$
n(\omega)=\sqrt{\epsilon(\omega)}=\sqrt{\epsilon^{\prime}} \cdot \sqrt{1+\frac{i \epsilon^{\prime \prime}}{\epsilon^{\prime}}} .
$$

In the limit that the imaginary component $\epsilon^{\prime \prime}$ is small compared to the real component, i.e., $\epsilon^{\prime \prime} \ll \epsilon^{\prime}$, Equation (B.2) can be linearized to

$$
n(\omega) \approx \sqrt{\epsilon^{\prime}}+\frac{i}{2} \frac{\epsilon^{\prime \prime}}{\sqrt{\epsilon^{\prime}}}=n_{b}+\frac{i}{2} \frac{\epsilon^{\prime \prime}}{n_{b}}
$$

where $n_{b}$ denotes a static background refractive index. The approximation in Equation (B.3) is valid for low carrier densities $\mathrm{N}_{d}$ like e.g., in semiconductors with low and intermediate doping concentrations. In addition, the approximation holds for rather high frequencies, due to the anti-proportional 
frequency dependence of the imaginary part of the dielectric function $\epsilon^{\prime \prime}$ which follows from Equation (B.1).

Samples investigated here had a doping concentration below $10^{17} \mathrm{~cm}^{-3}$. Assuming a scattering rate of $\tau=150 \mathrm{fs}, \epsilon^{\prime \prime} \ll \epsilon^{\prime}$ is always true for frequencies above the phonon resonance. Hence, the absorption coefficient of an optical wave reads:

$$
\alpha(\omega)=\frac{2 \omega n^{\prime \prime}(\omega)}{c} \approx \frac{N_{d} e}{n_{b} \epsilon_{0} c} \mu(\omega)
$$

where $n^{\prime \prime}(\omega)$ denotes the imaginary part of the refractive index. Equation (B.4) makes the link between the transport and the optical picture. The absorption coefficient is approximately linear to the electron mobility in the material under the present conditions. It should be stressed however, that the approximation fails for low frequencies, especially around the plasma resonance of the free electron gas, where the real part of the dielectric function is zero. 


\section{Appendix C}

\section{Electro-optic sampling without synchronization}

In conventional electro-optic sampling (Figure C.1 (a)), a THz or MIR pulse is scanned in time by an optical probe pulse. In order to increase the signal to noise ratio (SNR), each data point results from an averaging over consecutive probe pulses, which requires a synchronization of the measured signal, i.e., the $\mathrm{THz}$ or MIR transients, with the probe. In particular, synchronization means that consecutive transients are identical, that is $\mathrm{E}_{T H z}(\mathrm{t})=\mathrm{E}_{T H z}(\mathrm{t}+\mathrm{T})$ where $\mathrm{T}=1 / \mathrm{f}_{\mathrm{rep}}=1 / 71 \mathrm{MHz}$, i.e. the inverse of the pulse repetition rate.

Figure C.1 (b) shows the setup for an unsynchronized source. A measurement with such a setup will not yield the full information on the electric field. However, the following section shows that it can be used as a broadband frequency counter. In addition to the broad spectral region covered, which is determined by the bandwidth of the EOS detector, the presented method is particularly convenient for low frequencies which are difficult to resolve with upconversion techniques.

Figure C.2 (a) shows such a measurement on a linearly polarized cw $\mathrm{CO}_{2}$ laser emitting on the 10P20 line, which corresponds to a wavelength of $\approx 10.6 \mu \mathrm{m}$. [204]. The left side of this figure depicts the noise floor and the right side is a measurement with the $\mathrm{CO}_{2}$ laser incident on the detector. The signal to noise ratio (SNR) is approximately one. Knowing the laser power and the focal spot size, an electric field present on the detection crystal of $\mathrm{E}_{\mathrm{CO}_{2}} \approx 1 \mathrm{kV} / \mathrm{cm}$ was deduced. This value denotes the power resolution limit of the electro-optic sampling detection, resulting from the shot noise due to the finite amount of photons in the optical probe pulse. Higher sensitivities can be obtained by averaging over consecutive probe pulses, which would require a synchronization to the source of the measured radiation. In the synchronized case $\mathrm{SNR} \propto \sqrt{n_{\mathrm{avg}}}$, where $\mathrm{n}_{\mathrm{avg}}$ is the averaging number. 


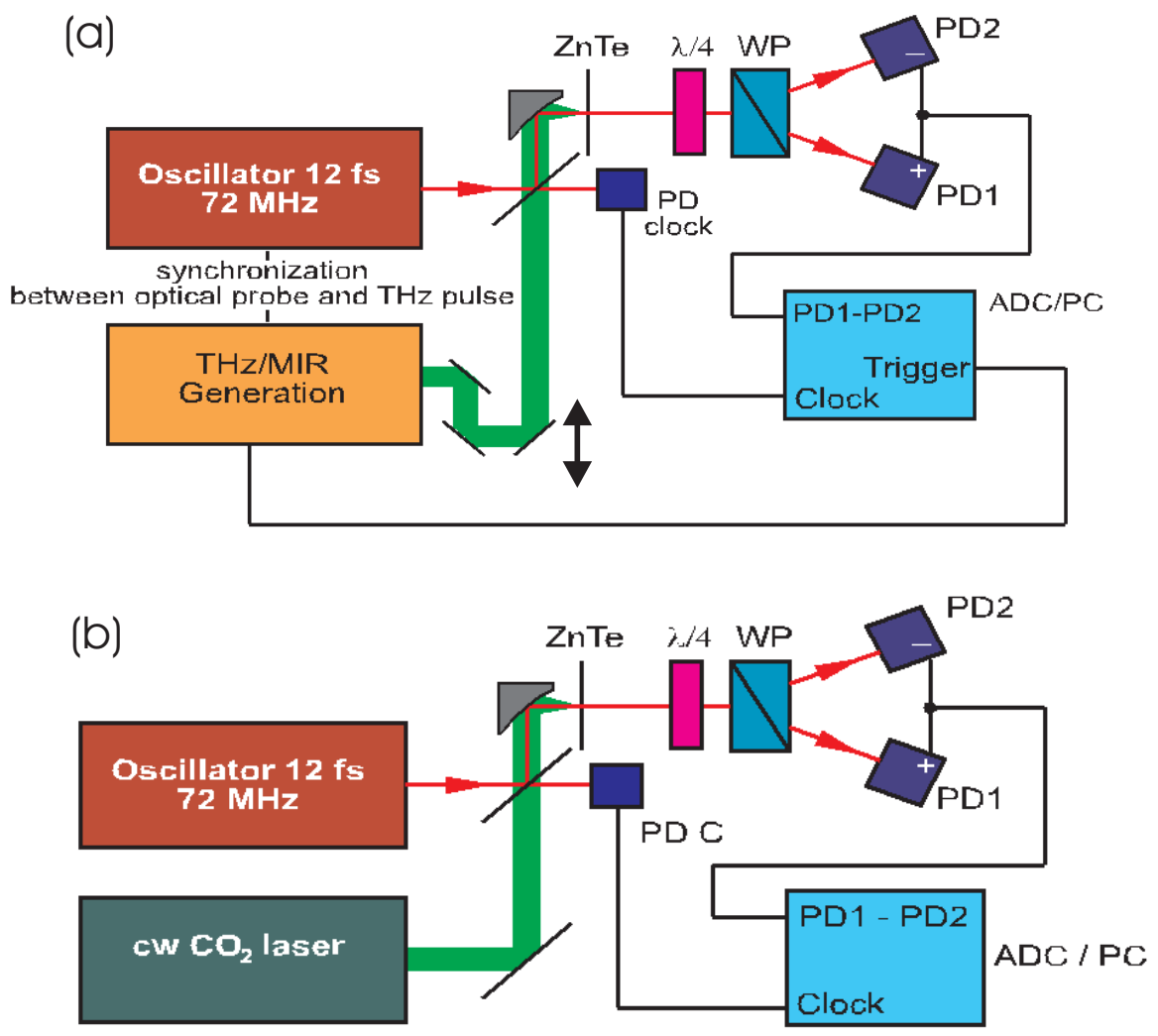

Figure C.1: (a) Synchronized EOS: A coherent pulsed MIR/THz source is measured via the EO effect in a ZnTe crystal. The difference signal PD1-PD2, which is proportional to the electric field of the $\mathrm{MIR} / \mathrm{THz}$ pulse is recorded using a highspeed analog-to-digital converter (ADC) and averaged for each temporal position. A delay line allows for sampling of the pump pulse according to Figure 3.3 (c). (b) Unsynchronized EOS of a $\mathrm{CO}_{2}$ Laser in $\mathrm{cw}$ mode. The high speed ADC records the difference signal $P D 1-P D 2 \neq 0$ in a single shot measurement. 


\section{Frequency $(\mathrm{THz})$}
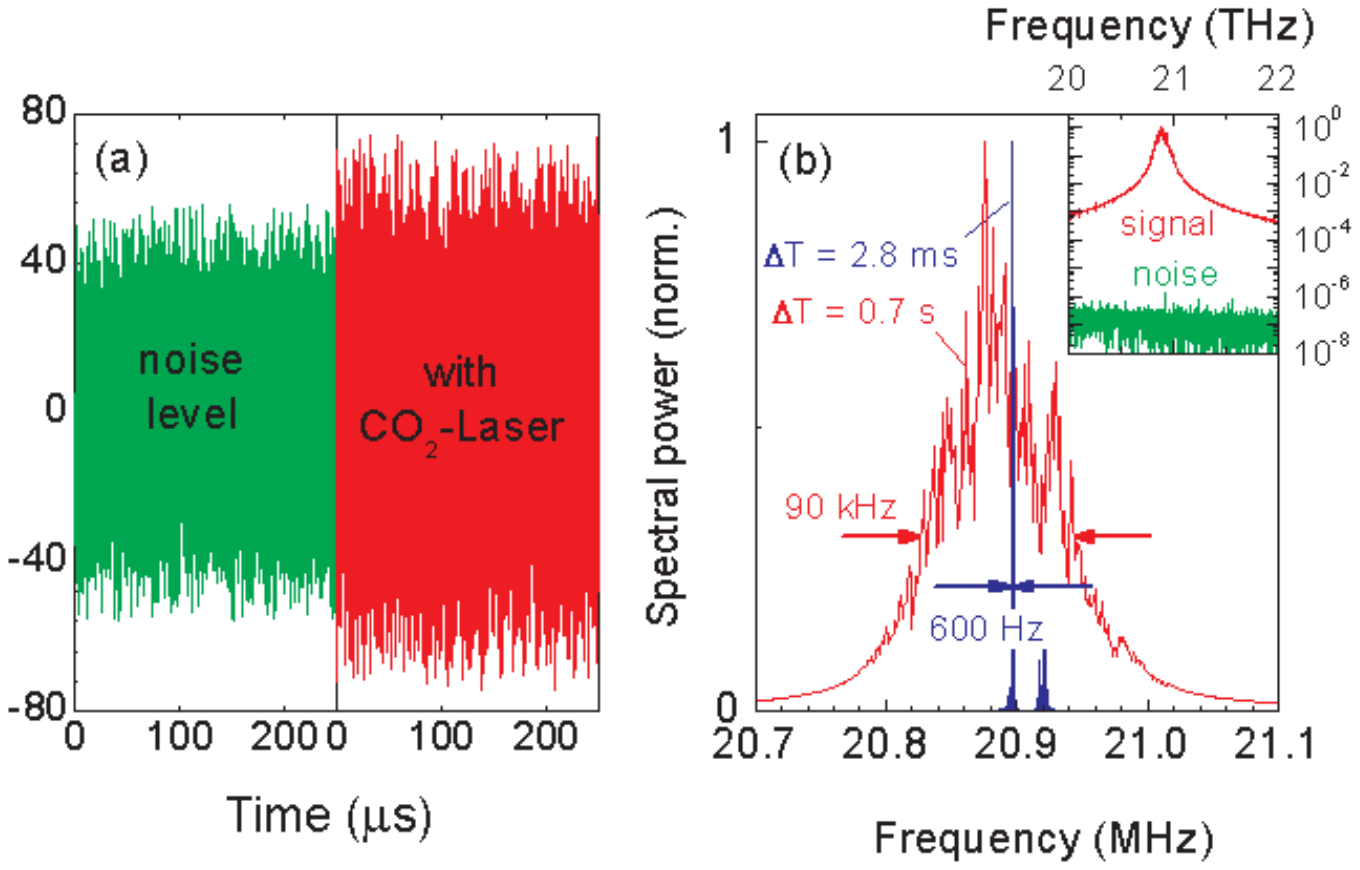

Figure C.2: (a) Electro-optic signal measured without (left green line) and with (right red line) the $\mathrm{CO}_{2}$ laser. (b) Spectra for a measurement time of $\Delta \mathrm{T}=0.7 \mathrm{~s}$ (red) and $\Delta \mathrm{T}=2.8 \mathrm{~ms}$ (blue). The inset shows the data for $\Delta \mathrm{T}=0.7 \mathrm{~s}$ again, together with the spectrum of the noise (green).

Autocorrelation spectra from unsynchronized measurements of two different duration are shown in Figure C.2 (b). A long measurement time yields a rather broad autocorrelation spectrum compared to short measurement times. In addition a fluctuation of the peak frequency was observed between consecutive identical measurements.

The linewidth of the observed peak at frequency $\mathrm{f}_{C}$ directly yields the linewidth of the electric field at the optical frequency $\nu$. To obtain the optical frequency $\nu$, one has to account for the undersampling of the electric field oscillating with frequency $\nu$ with a sampling rate of $\mathrm{f}_{\text {rep }}$, which is far below the Nyquist limit of $2 \nu$ [201]. Therefore $\mathrm{f}_{C}$ is given by $\mathrm{f}_{C}=\min \left(\mathrm{f}_{C}^{\prime}, \mathrm{f}_{\mathrm{rep}}-\mathrm{f}_{C}^{\prime}\right)$ with $\mathrm{f}_{C}^{\prime}=\nu \bmod \mathrm{f}_{\mathrm{rep}}$. Inversion yields

$$
\nu=n \times f_{\text {rep }} \pm f_{C},
$$

where $n$ is an integer. Equation (C.1) requires the determination of $\mathrm{n}$ and the proper sign for $f_{C}$. If these parameters are not known with sufficient accuracy beforehand, additional measurements with slightly different $f_{\text {rep }}$ (e.g. by changing the oscillator cavity length) have to be carried out. This 
would yield two equations for two unknown parameters, allowing to solve Equation (C.1) without ambiguity.

In the $\mathrm{CO}_{2}$ laser measurement the spectral peak for $\Delta \mathrm{T}=0.7 \mathrm{~s}$ (red line in Figure C.2(b)) is at $\mathrm{f}_{C}=20.88 \mathrm{MHz}$. Knowing that the laser operates on the 10P20 line $\mathrm{n}=395401$ and the positive sign for $\mathrm{f}_{C}$ in Equation (C.1) must be chosen. With $\mathrm{f}_{\mathrm{rep}}=71.586602 \mathrm{MHz}$ one finds for the optical frequency $\nu=28306225.7 \mathrm{MHz}$. The main source for the linewidths measured are fluctuations of the length of the $\mathrm{CO}_{2}$ laser resonator, of the Ti:sapphire laser resonator, or of both [205]. A thermal stabilization for both lasers would yield much narrower linewidths in the measurement. Even though the setup can be used as a frequency counter in the range of 1-40 THz, the measurement bandwidth is limited to $f_{\text {rep }} / 2$. Increasing the repetition rate would therefore be an additional improvement of the setup [206]. 


\section{Appendix D}

\section{Polarization dynamics in an ensemble of radiatively coupled two-level systems}

The Maxwell-Bloch equations introduced in Section 2.1.4 read

$$
\begin{aligned}
& \frac{\partial \varrho_{12}}{\partial t}=\left(i \omega_{0}-\frac{1}{T_{2}}\right) \varrho_{12}-i \frac{\vec{d}}{\hbar} E(t)\left(1-2 \varrho_{22}\right) \\
& \frac{\partial \varrho_{22}}{\partial t}=-\frac{\varrho_{22}}{T_{1}}-i \frac{\vec{d}}{\hbar} E(t) \operatorname{Im}\left(\varrho_{12}\right)
\end{aligned}
$$

The diagonal element $\varrho_{12}$ denotes the polarization in the system and $\varrho_{22}$ stands for the population of the upper level. In a quasi two-dimensional sample the the carriers which experience an incident electric field are radiatively coupled. Then, the macroscopic polarization in the sample reads

$$
P=2 N_{d} \overrightarrow{d d} \operatorname{Re}\left(\varrho_{12}\right)
$$

and the field emitted from the sample is given by the expression

$$
E_{e m}=-\frac{1}{2 \epsilon_{0} c} \frac{\partial P}{\partial t}=-\frac{N_{d} \vec{d} d}{\epsilon_{0} c} \frac{\partial R e\left(\varrho_{12}\right)}{\partial t}
$$

such that the local field $\mathrm{E}(\mathrm{t})$ acting on the sample is given by

$$
E(t)=E_{i n}(t)+E_{e m}(t)=E_{i n}(t)-\frac{N_{d} \overrightarrow{d d}}{\epsilon_{0} c} \frac{\partial R e\left(\varrho_{12}\right)}{\partial t}
$$

The following analysis is limited to quasi-stationary population, i.e., $\partial \varrho_{22} / \partial t=$

0 . To derive the equation of motion for the polarization, the off-diagonal elements of the density matrix are decomposed into their real and imaginary 
components:

$$
\varrho_{12}=(a+i b)
$$

In addition it is convenient to introduce the following abbreviations:

$$
\frac{\partial x}{\partial t}=\dot{x} \quad \gamma_{1}=\frac{\vec{d}}{\hbar} \quad \gamma_{2}=\frac{N_{d} \vec{d}^{2} d}{\hbar \epsilon_{0} c}
$$

Insertion of (D.6) and (D.7) into Equation (D.1) yields

$$
\begin{aligned}
(a+i b)^{\cdot}= & \left(i \omega_{0}-\frac{1}{T_{2}}\right)(a+i b)-\left(i \gamma_{1} E_{i n}+i \gamma_{2} \dot{a}\right)\left(1-2 \varrho_{22}\right) \\
= & i \omega_{0} a-\frac{a}{T_{2}}-b \omega_{0}-\frac{i b}{T_{2}}-i \gamma_{1}+i \gamma_{2} \dot{a}+i \gamma_{1} E_{i n} 2 \varrho_{22} \\
& -i \gamma_{2} 2 \varrho_{22} \dot{a}
\end{aligned}
$$

The real part reads

$$
\dot{a}=-\frac{a}{T_{2}}-b \omega_{0}
$$

and for the imaginary part one finds

$$
\dot{b}=\omega_{0} a-\frac{b}{T_{2}}-\gamma_{2} \dot{a}\left(1-2 \varrho_{22}\right)+\gamma_{1} E_{i n}\left(1-2 \varrho_{22}\right)
$$

Equation D.9 yields the relations

$$
b=-\frac{\dot{a}}{\omega_{0}}+\frac{a}{\omega_{0} T_{2}} \quad \dot{b}=-\frac{\ddot{a}}{\omega_{0}}+\frac{\dot{a}}{\omega_{0} T_{2}}
$$

After insertion of (D.11) in (D.10)

$$
-\frac{\ddot{a}}{\omega_{0}}+\frac{\dot{a}}{\omega_{0} T_{2}}=\omega_{0} a+\frac{\dot{a}}{\omega_{0} T_{2}}-\frac{a}{\omega_{0} T_{2}^{2}}+\gamma_{2} \dot{a}\left(1-2 \varrho_{22}\right)-\gamma_{1} E_{i n}\left(1-2 \varrho_{22}\right)
$$

one obtains the equation of motion of the polarization

$$
\ddot{a}=\left(-\omega_{0}^{2}+\frac{1}{T_{2}^{2}}\right) a-\left(\frac{2}{T_{2}}+\omega_{0} \gamma_{2}\left(1-2 \varrho_{22}\right)\right) \dot{a}+\omega_{0} \gamma_{1} E_{\text {in }}\left(1-2 \varrho_{22}\right)
$$

The damping term due to radiative coupling reads

$$
\frac{1}{2} \omega_{0} \gamma_{2}=\omega_{0} \frac{N_{d} \vec{d}_{1 S \rightarrow 2 p}^{2} d}{2 \hbar \epsilon_{0} c}=\Gamma_{r a d}
$$

In the linear case, i.e., $\varrho_{22}=0$, the radiative coupling decreases the polarization components of the Bloch vector, similar to the dephasing. In contrast, in the case of a complete population inversion, i.e., $\varrho_{22}=1$, the radiative coupling constant in Equation D.13 changes its sign. Hence, the radiative coupling tends to increase the polarization, i.e., it introduces a negative dephasing. 


\section{Appendix E}

\section{Derivation of the Heisenberg equations of motion}

In this appendix, the Heisenberg equation of motion (EOM)

$$
\frac{d A}{d t}=\frac{i}{\hbar}[H, A]
$$

is solved for $x, p, Q, P$, which represent the quantum mechanical operators for the electron position and momentum and the coordinate and its conjugated momentum of the LO phonon mode with wavevector $\vec{q}$, respectively. The Hamiltonian of an electron in a polar lattice with an external electric field and electron-phonon coupling reads:

$$
\begin{aligned}
H(t) & =\underbrace{\frac{\vec{p}^{2}}{2 m}}_{I}+\underbrace{e x E_{\mathrm{loc}}(t)}_{I I}+\underbrace{\sum_{\vec{q}} \frac{P_{\vec{q}}^{2}+\omega_{L O}^{2} Q_{\vec{q}}^{2}}{2}}_{I I I} \\
+ & \underbrace{\sqrt{\frac{e^{2}}{V \epsilon_{0}}\left(\frac{1}{\epsilon_{\infty}}-\frac{1}{\epsilon_{s}}\right)} \sum_{\vec{q}} \frac{P_{\vec{q}} \cos (\vec{q} \cdot \vec{r})+\omega_{L O} Q_{\vec{q}} \sin (\vec{q} \cdot \vec{r})}{|\vec{q}|}}_{I V} .
\end{aligned}
$$


In the EOM for the electron position $\mathrm{x}$ all but the first term of the Hamiltonian (E.2) vanish in the commutator $[H, x]$. Therefore, the EOM reads:

$$
\begin{aligned}
\frac{d x}{d t} & =\frac{i}{\hbar}[H, x]=-\frac{1}{i \hbar 2 m}\left[\frac{\partial^{2}}{\partial x^{2}} x-x \frac{\partial^{2}}{\partial x^{2}}\right] \\
& =-\frac{1}{i \hbar 2 m}\left[\frac{\partial}{\partial x}\left(x \frac{\partial}{\partial x}+1\right)-x \frac{\partial^{2}}{\partial x^{2}}\right] \\
& =-\frac{1}{i \hbar 2 m}\left[x \frac{\partial^{2}}{\partial x^{2}}+\frac{\partial}{\partial x}+\frac{\partial}{\partial x}-x \frac{\partial^{2}}{\partial x^{2}}\right] \\
& =\frac{1}{i \hbar 2 m}\left[2 \frac{\partial}{\partial x}\right] \\
& =\frac{p}{m}=v .
\end{aligned}
$$

Next the EOM for the electron momentum $\mathrm{p}$ is derived. Here the terms (II) and (IV) of equation (E.2) are the non-vanishing terms.

$$
\begin{aligned}
\frac{d p_{x}}{d t}= & \frac{i}{\hbar}\left[H, p_{x}\right] \\
= & {\left[\left(e x E_{\mathrm{loc}}(t)+\sqrt{A} \sum_{\vec{q}} \frac{P_{\vec{q}} \cos (\vec{q} \cdot \vec{r})+\omega_{L O} Q_{\vec{q}} \sin (\vec{q} \cdot \vec{r})}{|\vec{q}|}\right) \frac{\partial}{\partial x}\right.} \\
& \left.-\frac{\partial}{\partial x}\left(e x E_{\mathrm{loc}}(t)+\sqrt{A} \sum_{\vec{q}} \frac{P_{\vec{q}} \cos (\vec{q} \cdot \vec{r})+\omega_{L O} Q_{\vec{q}} \sin (\vec{q} \cdot \vec{r})}{|\vec{q}|}\right)\right] \\
= & {\left[\left(e x E_{\mathrm{loc}}(t)+\sqrt{A} \sum_{\vec{q}} \frac{P_{\vec{q}} \cos (\vec{q} \cdot \vec{r})+\omega_{L O} Q_{\vec{q}} \sin (\vec{q} \cdot \vec{r})}{|\vec{q}|}\right) \frac{\partial}{\partial x}\right.} \\
& -\left(e E_{\mathrm{loc}}(t)+\sqrt{A} \sum_{\vec{q}} \frac{-P_{\vec{q}} q_{x} \sin (\vec{q} \cdot \vec{r})+\omega_{L O} q_{x} Q_{\vec{q}} \cos (\vec{q} \cdot \vec{r})}{|\vec{q}|}\right) \\
& \left.-\left(e x E_{\mathrm{loc}}(t)+\sqrt{A} \sum_{\vec{q}} \frac{P_{\vec{q}} \cos (\vec{q} \cdot \vec{r})+\omega_{L O} Q_{\vec{q}} \sin (\vec{q} \cdot \vec{r})}{|\vec{q}|}\right) \frac{\partial}{\partial x}\right] \\
= & \left(-e E_{\mathrm{loc}}(t)+\sqrt{A} \sum_{\vec{q}} \frac{q_{x} P_{\vec{q}} \sin (\vec{q} \cdot \vec{r})+\omega_{L O} q_{x} Q_{\vec{q}} \cos (\vec{q} \cdot \vec{r})}{|\vec{q}|}\right),
\end{aligned}
$$

where

$$
\sqrt{A}=\sqrt{\frac{e^{2}}{V \epsilon_{0}}\left(\frac{1}{\epsilon_{\infty}}-\frac{1}{\epsilon_{s}}\right)} .
$$

Now the EOM for the canonical operators $Q_{\vec{q}}$ and $P_{\vec{q}}=-i \hbar \partial / \partial Q_{\vec{q}}$ are derived. From equation (E.2) terms (III) and (IV) yield a contribution. For 
the first operator, the EOM reads:

$$
\begin{aligned}
\frac{d Q_{\vec{q}}}{d t}= & \frac{i}{\hbar}\left[H, Q_{\vec{q}}\right] \\
= & \frac{i}{\hbar}\{\underbrace{\left[\left(\frac{P_{\vec{q}}^{2}+\omega_{L O}^{2} Q_{\vec{q}}^{2}}{2}+\sqrt{A} \frac{P_{\vec{q}} \cos (\vec{q} \cdot \vec{r})+\omega_{L O} Q_{\vec{q}} \sin (\vec{q} \cdot \vec{r})}{|\vec{q}|}\right) Q_{\vec{q}}\right]}_{\alpha} \\
& -\underbrace{\left[Q_{\vec{q}}\left(\frac{P_{\vec{q}}^{2}+\omega_{L O}^{2} Q_{\vec{q}}^{2}}{2}+\sqrt{A} \frac{P_{\vec{q}} \cos (\vec{q} \cdot \vec{r})+\omega_{L O} Q_{\vec{q}} \sin (\vec{q} \cdot \vec{r})}{|\vec{q}|}\right)\right]}_{\beta} .
\end{aligned}
$$

Solving term $(\alpha)$ yields:

$$
\begin{aligned}
& {\left[\frac{\left(-i \hbar \partial / \partial Q_{\vec{q}}\right)^{2}+\omega_{L O}^{2} Q_{\vec{q}}^{2}}{2}+\sqrt{A} \frac{\left(-i \hbar \partial / \partial Q_{\vec{q}}\right) \cos (\vec{q} \cdot \vec{r})+\omega_{L O} Q_{\vec{q}} \sin (\vec{q} \cdot \vec{r})}{|\vec{q}|}\right] Q_{\vec{q}} } \\
= & \frac{\left(-i \hbar \partial / \partial Q_{\vec{q}}\right)^{2} Q_{\vec{q}}}{2}+\frac{\omega_{L O}^{2} Q_{\vec{q}}^{3}}{2}+\sqrt{A} \frac{i \hbar \partial / \partial Q_{\vec{q}} \cos () Q_{\vec{q}}}{|\vec{q}|}+\sqrt{A} \frac{\omega_{L O} Q_{\vec{q}}^{2} \sin (\vec{q} \cdot \vec{r})}{|\vec{q}|} \\
= & B+\left[\left(\frac{-\hbar^{2}}{2} \frac{\partial}{\partial Q_{\vec{q}}}+\sqrt{A} \frac{-i \hbar \cos (\vec{q} \cdot \vec{r})}{|\vec{q}|}\right)\left(1+Q_{\vec{q}} \frac{\partial}{\partial Q}\right)\right] \\
= & B+\left[-\frac{\hbar^{2}}{2}\left(2 \frac{\partial}{\partial Q}+Q_{\vec{q}} \frac{\partial^{2}}{\partial Q^{2}}\right)+\sqrt{A} \frac{i \hbar \cos (\vec{q} \cdot \vec{r})}{|\vec{q}|}\left(1+Q_{\vec{q}} \frac{\partial}{\partial Q}\right)\right],
\end{aligned}
$$

where

$$
B=\sum_{\vec{q}}\left[\frac{\omega_{L O}^{2} Q_{\vec{q}}^{3}}{2}+\sqrt{A} \frac{\omega_{L O} Q_{\vec{q}}^{2} \sin (\vec{q} \cdot \vec{r})}{|\vec{q}|}\right] .
$$

Term ( $\beta)$ yields:

$$
\begin{aligned}
& Q_{\vec{q}}\left(\frac{\left(-i \hbar \partial / \partial Q_{\vec{q}}\right)^{2}+\omega_{L O}^{2} Q_{\vec{q}}^{2}}{2}+\sqrt{A} \frac{-i \hbar \partial / \partial Q_{\vec{q}} \cos (\vec{q} \cdot \vec{r})+\omega_{L O} Q_{\vec{q}} \sin (\vec{q} \cdot \vec{r})}{|\vec{q}|}\right) \\
= & B+Q_{\vec{q}} \frac{-\hbar^{2} \partial^{2} / \partial Q_{\vec{q}}^{2}}{2}+\sqrt{A} \frac{-i \hbar Q_{\vec{q}} \cos (\vec{q} \cdot \vec{r}) \partial / \partial Q_{\vec{q}}}{|\vec{q}|} \\
= & B+\frac{-\hbar^{2}}{2} Q_{\vec{q}} \frac{\partial^{2}}{\partial Q^{2}}+\sqrt{A} \frac{-i \hbar \cos (\vec{q} \cdot \vec{r})}{|\vec{q}|} Q_{\vec{q}} \frac{\partial}{\partial Q} .
\end{aligned}
$$


The EOM for the operator $Q_{\vec{q}}$ is:

$$
\begin{aligned}
\frac{d Q_{\vec{q}}}{d t}= & \frac{i}{\hbar}\left[H, Q_{\vec{q}}\right]=\frac{i}{\hbar}[(\alpha)-(\beta)] \\
= & \frac{i}{\hbar}\left\{B+\frac{-\hbar^{2}}{2}\left(2 \frac{\partial}{\partial Q}+Q_{\vec{q}} \frac{\partial^{2}}{\partial Q^{2}}\right)+\sqrt{A} \frac{i \hbar \cos (\vec{q} \cdot \vec{r})}{|\vec{q}|}\left(1+q \frac{\partial}{\partial Q}\right)\right. \\
& \left.-\left[B+\frac{-\hbar^{2}}{2} Q_{\vec{q}} \frac{\partial^{2}}{\partial Q^{2}}+\sqrt{A} \frac{i \hbar \cos (\vec{q} \cdot \vec{r})}{|\vec{q}|}\left(Q_{\vec{q}} \frac{\partial}{\partial Q}\right)\right]\right\} \\
= & \frac{i}{\hbar}\left\{-\hbar^{2} \partial / \partial Q_{\vec{q}}+\sqrt{A} \frac{i \hbar \cos (\vec{q} \cdot \vec{r})}{|\vec{q}|}\right\} \\
= & P_{\vec{q}}+\sqrt{A} \sum_{q} \frac{\cos (\vec{q} \cdot \vec{r})}{|\vec{q}|} .
\end{aligned}
$$

It remains to derive the EOM for the operator $P$ :

$$
\begin{aligned}
\frac{d P_{\vec{q}}}{d t}= & \frac{i}{\hbar}\left[H, P_{\vec{q}}\right] \\
= & \frac{i}{\hbar}\{\underbrace{\left[\left(\frac{P_{\vec{q}}^{2}}{2}+\frac{\omega_{L O}^{2} Q_{\vec{q}}^{2}}{2}+\sqrt{A} \frac{P_{\vec{q}} \cos (\vec{q} \cdot \vec{r})}{|\vec{q}|}+\sqrt{A} \frac{\omega_{L O} Q_{\vec{q}} \sin (\vec{q} \cdot \vec{r})}{|\vec{q}|}\right) P_{\vec{q}}\right]}_{(\gamma)} \\
& -\underbrace{\left[P_{\vec{q}}\left(\frac{P_{\vec{q}}^{2}}{2}+\frac{\omega_{L O}^{2} Q_{\vec{q}}^{2}}{2}+\sqrt{A} \frac{P_{\vec{q}} \cos (\vec{q} \cdot \vec{r})}{|\vec{q}|}+\sqrt{A} \frac{\omega_{L O} Q_{\vec{q}} \sin (\vec{q} \cdot \vec{r})}{|\vec{q}|}\right)\right]}_{(\delta)}\} .
\end{aligned}
$$

Solving term $(\gamma)$ yields:

$$
(\gamma)=C+\frac{\omega_{L O}^{2} Q_{\vec{q}}^{2}}{2} \frac{\partial}{\partial Q}+\sqrt{A} \frac{-i \hbar \omega_{L O} Q_{\vec{q}} \sin (\vec{q} \cdot \vec{r})}{|\vec{q}|} \frac{\partial}{\partial Q},
$$

where

$$
C=\frac{1}{2} \frac{\partial^{3}}{\partial Q_{\vec{q}}^{3}}+\sqrt{A} \frac{\partial^{2} / \partial Q_{\vec{q}} \cos (\vec{q} \cdot \vec{r})}{|\vec{q}|} .
$$

Term $(\delta)$ reads:

$$
\begin{aligned}
(\delta)= & C-\frac{-i \hbar \omega_{L O}^{2}}{2}\left(2 Q_{\vec{q}}+Q_{\vec{q}}^{2} \frac{\partial}{\partial Q}\right) \\
& -\sqrt{A} \frac{-i \hbar \omega_{L O} \sin (\vec{q} \cdot \vec{r})}{|\vec{q}|}\left(1+Q \frac{\partial}{\partial Q}\right) .
\end{aligned}
$$


Hence, the EOM for the phonon momentum reads:

$$
\begin{aligned}
\frac{d P_{\vec{q}}}{d t}= & \frac{i}{\hbar}\left[H, P_{\vec{q}}\right]=\frac{i}{\hbar}[(\gamma)-(\delta)]= \\
= & \frac{i}{\hbar}\left\{C+\frac{-i \hbar \omega_{L O}^{2} Q_{\vec{q}}^{2}}{2} \frac{\partial}{\partial Q}+\sqrt{A} \frac{-i \hbar \omega_{L O} Q_{\vec{q}} \sin (\vec{q} \cdot \vec{r})}{|\vec{q}|} \frac{\partial}{\partial Q}\right. \\
& \left.-\left[C+\frac{-i \hbar \omega_{L O}^{2}}{2}\left(2 Q_{\vec{q}}+Q_{\vec{q}}^{2} \frac{\partial}{\partial Q}\right)+\sqrt{A} \frac{-i \hbar \omega_{L O} \sin (\vec{q} \cdot \vec{r})}{|\vec{q}|}\left(1+Q_{\vec{q}} \frac{\partial}{\partial Q}\right)\right]\right\} \\
= & -\omega_{L O}^{2} Q_{\vec{q}}-\sqrt{A} \frac{\omega_{L O} \sin (\vec{q} \cdot \vec{r})}{|\vec{q}|}
\end{aligned}
$$




\section{Appendix F}

\section{Taylor expansion of the expectation value $\left\langle P_{\vec{q}} \sin (\vec{q} \cdot \vec{r})\right\rangle$}

In order to close the infinite hierarchy of equations of motions of the conduction band electron propagating in the external electric field, the expectation values of the operators $\mathrm{P}_{\vec{q}} \sin (\vec{q} \cdot \vec{r}), \mathrm{Q}_{\vec{q}} \cos (\vec{q} \cdot \vec{r}), \sin (\vec{q} \cdot \vec{r})$ and $\cos (\vec{q} \cdot \vec{r})$ are expanded in a Taylor series which is truncated at the second order. For example, the expansion of an operator $\mathrm{F}(\mathrm{x})$ is:

$$
\langle F(x)\rangle=F(\langle x\rangle)+\langle(x-\langle x\rangle)\rangle\left(\frac{\partial F}{\partial x}\right)_{x=\langle x\rangle}+\frac{1}{2 !}\left\langle(x-\langle x\rangle)^{2}\right\rangle\left(\frac{\partial^{2} F}{\partial x^{2}}\right)_{x=\langle x\rangle}
$$

Here, the second Taylor expansion is demonstrated as an example for the operator $\left\langle P_{\vec{q}} \sin (\vec{q} \cdot \vec{r})\right\rangle=\left\langle P_{\vec{q}} \sin \left(q_{x} x+q_{y} y+q_{z} z\right)\right\rangle$ around the expectation values $P_{\vec{q}}=\left\langle P_{\vec{q}}\right\rangle, x=\langle x\rangle, y=\langle y\rangle, z=\langle z\rangle$. The resulting expression is simplified by applying symmetry relations and approximations which will be detailed in the following. 


$$
\begin{aligned}
&\left\langle P_{\vec{q}} \sin (\vec{q} \cdot \vec{r})\right\rangle= \\
&+\left\langle P_{\vec{q}}\right\rangle \sin \left(q_{x}\langle x\rangle+q_{y}\langle y\rangle+q_{z}\langle z\rangle\right) \\
&+\left\langleP _ { \vec { q } } \left[ q_{x} \cos \left(q_{x} x+q_{y} y+q_{z} z\right)(x-\langle x\rangle)\right.\right. \\
& \quad+q_{y} \cos \left(q_{x} x+q_{y} y+q_{z} z\right)(y-\langle y\rangle) \\
&\left.\left.\quad+q_{z} \cos \left(q_{x} x+q_{y} y+q_{z} z\right)(z-\langle z\rangle)\right]\right\rangle \\
&-\left\langleP _ { \vec { q } } \left[\frac{q_{x}^{2}}{2} \sin \left(q_{x} x+q_{y} y+q_{z} z\right)(x-\langle x\rangle)^{2}\right.\right. \\
& \quad+\frac{q_{y}^{2}}{2} \sin \left(q_{x} x+q_{y} y+q_{z} z\right)(y-\langle y\rangle)^{2} \\
& \quad+\frac{q_{z}^{2}}{2} \sin \left(q_{x} x+q_{y} y+q_{z} z\right)(z-\langle z\rangle)^{2} \\
& \quad+q_{x} q_{y} \sin \left(q_{x} x+q_{y} y+q_{z} z\right)(x-\langle x\rangle)(y-\langle y\rangle) \\
& \quad+q_{x} q_{z} \sin \left(q_{x} x+q_{y} y+q_{z} z\right)(x-\langle x\rangle)(z-\langle z\rangle) \\
&\left.\left.\quad+q_{y} q_{z} \sin \left(q_{x} x+q_{y} y+q_{z} z\right)(y-\langle y\rangle)(z-\langle z\rangle)\right]\right\rangle \\
&+\left\langle q_{x} \cos \left(q_{x} x+q_{y} y+q_{z} z\right)(x-\langle x\rangle)\left(P_{\vec{q}}-\left\langle P_{\vec{q}}\right\rangle\right)\right\rangle \\
&+\left\langle q_{y} \cos \left(q_{x} x+q_{y} y+q_{z} z\right)(y-\langle y\rangle)\left(P_{\vec{q}}-\left\langle P_{\vec{q}}\right\rangle\right)\right\rangle \\
&+\left\langle q_{z} \cos \left(q_{x} x+q_{y} y+q_{z} z\right)(z-\langle z\rangle)\left(P_{\vec{q}}-\left\langle P_{\vec{q}}\right\rangle\right)\right\rangle \\
&+\cdots
\end{aligned}
$$

The zero order term (F.3) is the classical approximation which follows from the theorem of Ehrenfest. The first order terms (F.4)-(F.6) vanish because

$$
\langle n-\langle n\rangle\rangle=\langle n\rangle-\langle n\rangle=0,
$$

where $\mathrm{n}$ denotes the coordinates $\mathrm{x}, \mathrm{y}$ and $\mathrm{z}$, respectively.

Since the electron is accelerated by a linearly polarized electric field $\mathrm{E}_{x}$, the problem has cylindrical symmetry. Therefore the following expectation values also vanish:

$$
\begin{aligned}
\langle y\rangle & =0, & \langle z\rangle & =0, \\
\langle x y\rangle & =0, & \langle x z\rangle & =0, \\
\left\langle P_{\vec{q}} y\right\rangle & =0, & \left\langle P_{\vec{q}} z\right\rangle & =0 .
\end{aligned}
$$

The mixed factors of the second order expansion terms read e.g.

$$
\langle(x-\langle x\rangle)(y-\langle y\rangle)\rangle=(\langle x y\rangle-\langle x\rangle\langle y\rangle) .
$$


With the identities (F.18) the terms (F.14)-(F.15), (F.10)-(F.12) plus the $\langle y\rangle$ - and $\langle z\rangle$-terms in the argument of the sine and cosine function are ruled out. In addition, in equilibrium, i.e., without an external field, the polaron has spherical symmetry. Therefore the term (F.13) is zero.

The remaining second order terms simplify to

$$
(x-\langle x\rangle)^{2}=\left\langle x^{2}-2\langle x\rangle\langle x\rangle+\langle x\rangle^{2}\right\rangle=\left\langle x^{2}\right\rangle-\langle x\rangle^{2} .
$$

In the following, $\langle x(t=0)\rangle=0$ is assumed without loss of generality. In addition, a spherical polaron is assumed, i.e.,

$$
\left\langle x^{2}\right\rangle-\langle x\rangle^{2}=\left\langle y^{2}\right\rangle=\left\langle z^{2}\right\rangle=R^{2},
$$

where $R^{2}$ is the constant polaron radius given by equation (2.38). The approximation of a constant, spherical polaron is only valid on ultrafast time scales.

Takeing all symmetry considerations and the spherical approximation into account, the Taylor expansion reads:

$$
\begin{aligned}
\left\langle P_{\vec{q}} \sin \left(q_{x} x+q_{y} y+q_{z} z\right)\right\rangle \approx & \\
& \left\langle P_{\vec{q}}\right\rangle \sin \left(q_{x}\langle x\rangle\right) \\
+ & \frac{q_{x}^{2}}{2} \sin \left(q_{x} x\right)\left(\left\langle x^{2}\right\rangle-\langle x\rangle^{2}\right) \\
+ & \frac{q_{y}^{2}}{2} \sin \left(q_{x} x\right)\left\langle y^{2}\right\rangle \\
+ & \frac{q_{z}^{2}}{2} \sin \left(q_{x} x\right)\left\langle z^{2}\right\rangle \\
& =\left\langle P_{\vec{q}}\right\rangle \sin \left(q_{x}\langle x\rangle\right)\left[1-\frac{\vec{q}^{2}}{2} R^{2}\right] .
\end{aligned}
$$

The term in parenthesis in equation (F.22) is the second order correction of the expectation value due to the finite size of the electron wavefunction.

Since

$$
\lim _{\vec{q}^{2} \rightarrow \infty}\left\langle P_{\vec{q}}\right\rangle \sin \left(q_{x}\langle x\rangle\right)\left[1-\frac{\vec{q}^{2}}{2} R^{2}\right]=-\infty
$$

equation (F.22) is approximated with an exponential decay, which simulates the neglected higher order terms of the Taylor expansion.

$$
\left\langle P_{\vec{q}}\right\rangle \sin \left(q_{x}\langle x\rangle\right)\left[1-\frac{\vec{q}^{2}}{2} R^{2}\right] \approx P_{\vec{q}} \sin \left(q_{x}\langle x\rangle\right) \cdot \exp \left[-\frac{\vec{q}^{2}}{2} R^{2}\right]
$$

The approximation is shown in Figure F.1. Note that equation (F.24) is 


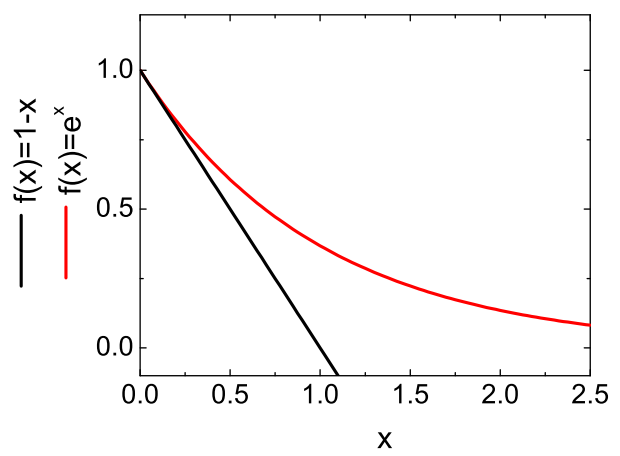

Figure F.1: Plot of the approximation function: $f(x)=[1-x]$ (black solid line) approximated by $f(x)=e^{-x}$ (red solid line). The approximation function reduces the coupling for higher values of $\mathrm{x}$ without extending to negative values.

strictly positive for all values $(\vec{q} \cdot \vec{r})$ but limits the coupling to phonons with higher $\vec{q}$-vector.

The operators $\mathrm{Q}_{\vec{q}} \cos (\vec{q} \cdot \vec{r}), \cos (\vec{q} \cdot \vec{r})$ and $\sin (\vec{q} \cdot \vec{r})$ are expanded in a similar way and read after expansion and approximation:

$$
\begin{aligned}
\left\langle Q_{\vec{q}} \cos (\vec{q} \cdot \vec{r})\right\rangle & =\left\langle Q_{\vec{q}}\right\rangle \cos \left(q_{x}\langle x\rangle\right) \cdot \exp \left[-\frac{\vec{q}^{2}}{2} R^{2}\right], \\
\langle\cos (\vec{q} \cdot \vec{r})\rangle & =\cos \left(q_{x}\langle x\rangle\right) \cdot \exp \left[-\frac{\vec{q}^{2}}{2} R^{2}\right] \\
\langle\sin (\vec{q} \cdot \vec{r})\rangle & =\sin \left(q_{x}\langle x\rangle\right) \cdot \exp \left[-\frac{\vec{q}^{2}}{2} R^{2}\right] .
\end{aligned}
$$




\section{Bibliography}

[1] Sze, S. M. ; Irvin, J. C.: Resistivity, Mobility and Impurity Levels in GaAs, Ge and Si at 300 K. In: Solid State Electron. 11 (1968), S. 599

[2] Shockely, W. ; Bardeen, J. ; Brattain, W. H.: The electronic theory of the transistor. In: Science 108 (1948), S. 678-679

[3] Moore, G. E.: Progress in digital integrated electronics. In: IEDM Tech. Dig. 21 (1975), S. 11-13

[4] INTEL, Corp.: www.intel.com. 2008

[5] Datta, S. ; Ashley, T. ; Brask, J. ; Buckle, L. ; Doczy, M. ; Hayes, D. ; Hilton, K. ; Jefferies, R. ; Martin, T. ; Phillips, T. J. ; Wallis, D. ; Wilding, P. ; Chau, R.: 85 nm Gate Length and Depletion mode in InSb Quantum Well Transistors for Ultra High Speed and Very Low Power Digital Logic Applications. 2005

[6] Barbieri, S. ; Alton, J. ; Dhillon, S. S. ; Beere, H. E. ; Evens, M. ; Linfield, E. H. ; Davis, A. G. ; Kohler, R. ; Tredicucci, A. ; Beltram, F.: Continous-wave operation of terahertz quantumcascade lasers. In: IEEE Journal of quantum electronics 39 (2003), S. $586-591$

[7] Faist, J. ; Capasso, F. ; Sivco, D. L. ; Hutchinson, A. L. ; Sirtori, C. ; Cho, A. Y.: Quantum cascade laser. In: Science 264 (1994), S. $553-556$

[8] Beard, M. C. ; Turner, G. M .. ; Schmuttenmaer, C. A.: Terahertz Spectroscopy. In: J. Phys. Chem. B 106 (2002), S. 7146-7159

[9] Cheliokowsky, J. R. ; Cohen, M. L.: Nonlocal pseudopotential calculations for the electronic structure of eleven diamond and zincblende semiconductors. In: Phys. Rev. B 14 (1976), S. 556-582 
[10] Blackmore, J. S.: Semiconducting and other major properties of gallium arsenide. In: J. appl. Phys. 53 (1982), S. R123-R181

[11] Sobiesierski, Z. ; Woolf, D. A. ; Westwood, D. I. ; Williams, R. H.: Photoluminescence measurements for GaAs grown on $\mathrm{Si}(100)$ and $\operatorname{Si}(111)$ by molecular beam epitaxy. In: App. Phys. Lett. 58 (1991), S. $628-630$

[12] Strauch, D. ; Dorner, B.: Phonon dispersion in GaAs. In: J. Phys.: Condens. Matter 12 (1990), S. 1457-1474

[13] Yu, P. Y. ; Cardona, M.: Fundamentals of Semiconductors. 3. Springer, 2005

[14] Seeger, K.: Semiconductor Physics. 5. Berlin : Springer, 1991

[15] Kittel, C.: Physik der Wärme. München : Oldenbourg, 1973

[16] Rogers, F. J. ; Graboske, H. C. ; Harwood, D. J.: Bound Eigenstates of the Static Screened Coulomb Potential. In: Phys. Rev. A 1 (1970), Jun, Nr. 6, S. 1577-1586. http://dx.doi.org/10.1103/ PhysRevA.1.1577. - DOI 10.1103/PhysRevA.1.1577

[17] Kittel, C.: Introduction to Solid State Physics. 7. New York: Wiley, 1996

[18] Rosenbaum, T. F. ; Andres, K. ; Thomas, G. A. ; Bhatt, R. N.: Sharp Metal-Insulator Transition in a Random Solid. In: Phys. Rev. Lett. 45 (1980), Nov, Nr. 21, S. 1723-1726. http://dx.doi.org/10. 1103/PhysRevLett.45.1723. - DOI 10.1103/PhysRevLett.45.1723

[19] Stupp, H. ; Hornung, M. ; Lakner, M. ; Madel, Q. ; LÖHneySEN, H. v.: Possible solution of the conductivity exponent puzzle for the metal-insulator transition in heavily doped uncompensated semiconductors. In: Phys. Rev. Lett. 71 (1993), Oct, Nr. 16, S. 26342637. http://dx.doi.org/10.1103/PhysRevLett.71.2634. - DOI 10.1103/PhysRevLett.71.2634

[20] Cohen-Tannoudu, C. ; Diu, B. ; Lalö̈, F.: Méchanique Quantique. Bd. II. Paris : Hermann, 1986

[21] Helm, M.: Infrared spectroscopy and transport of electrons in semiconductor superlattices. In: Semicond. Sci. Technol. 10 (1995), S. $557-575$ 
[22] Helm, M. ; Hilber, W. ; Fromherz, T. ; Peeters, F. M. ; Alavi, K. ; Pathak, R. N.: Infrared absorption in superlattices: A probe of the miniband dispersion and the structure of the impurity band. In: Phys. Rev. B 48 (1993), Jul, Nr. 3, S. 1601-1606. http://dx.doi. org/10.1103/PhysRevB.48.1601. - DOI 10.1103/PhysRevB.48.1601

[23] Anderson, P. W.: Absence of Diffusion in Certain Random Lattices. In: Phys. Rev. 109 (1958), Mar, Nr. 5, S. 1492-1505. http://dx.doi. org/10.1103/PhysRev.109.1492. - DOI 10.1103/PhysRev.109.1492

[24] Shat, J.: Ultrafast Spectroscopy of Semiconductors and Semiconductor Nanostructures. Berlin : Springer, 1996

[25] YArIv, A.: Quantum Electronics. 3. New York: Wiley, 1989

[26] Luo, C. W. ; Reimann, K. ; Woerner, M. ; Elsaesser, T. ; Hey, R. ; Ploog, K. H.: Phase-resolved nonlinear response of a twodimensional electron gas under femtosecond intersubband excitation. In: Phys. Rev. Lett. 92 (2004), S. 047402-1-4

[27] Pearson, G. L. ; Bardeen, J.: Electrical Properties of Pure Silicon and Silicon Alloys Containing Boron and Phosphorus. In: Phys. Rev. 75 (1949), Mar, Nr. 5, S. 865-883. http://dx.doi.org/10.1103/ PhysRev.75.865. - DOI 10.1103/PhysRev.75.865

[28] Kittel, C. ; Mitchell, A.H.: Theory of Donor and Acceptor States in Silicon and Germanium. In: Phys. Rev. 96 (1954), Dec, Nr. 6, S. 1488-1493. http://dx.doi.org/10.1103/PhysRev.96.1488. - DOI 10.1103/PhysRev.96.1488

[29] Luttinger, J. M. ; Kohn, W.: Motion of Electrons and Holes in Perturbed Periodic Fields. In: Phys. Rev. 97 (1955), Feb, Nr. 4, S. 869-883. http://dx.doi.org/10.1103/PhysRev.97.869. - DOI 10.1103/PhysRev.97.869

[30] Kohn, W. ; Schechter, D.: Theory of Acceptor Levels in Germanium. In: Phys. Rev. 99 (1955), Sep, Nr. 6, S. 1903-1904. http: //dx.doi.org/10.1103/PhysRev.99.1903. - DOI 10.1103/PhysRev.99.1903

[31] Klaasen, T. O. ; Dumm, J. L. ; Bates, C. A.: Shallow donor states in a magnetic field. In: Schmelcher, P. (Hrsg.): Atoms and molecules in strong external fields. New York : Plenum Press, 1998, S. 291-300 
[32] Cooke, R. A. ; Hoult, R. A. ; Kirkman, R. F. ; Stradling, R. A.: The characterisation of the donors in GaAs epitaxial films by far-infrared photoconductive techniques. In: J. Phys. D 11 (1978), S. $945-953$

[33] Stillmann, G. E. ; Wolfe, C. M. ; Melngailis, I. ; Parker, C. D. ; Tannenwald, P. E. ; Dimmock, J. O.: Far-infrared photocunductivity in high-purity epitaxal GaAs. In: Appl. Phys. Lett. 13 (1968), S. 83-84

[34] Koenig, S. H. ; Brown, R. D.: Far Infrared Electron-Ionized Donor Recombination Radiation in Germanium. In: Phys. Rev. Lett. 4 (1960), S. 170

[35] Hrostowski, H. J. ; Kaiser, R. H.: Infrared spectra of group III acceptors in silicon. In: J. Phys. Chem. Solids 4 (1957), S. 148-153

[36] Zwerdling, S. ; Button, K. J. ; Lax, B. ; Roth, L. M.: Internal Impurity Levels in Semiconductors: Experiments in $p$-Type Silicon. In: Phys. Rev. Lett. 4 (1960), Feb, Nr. 4, S. 173-176. http://dx.doi.org/ 10.1103/PhysRevLett.4.173. - DOI 10.1103/PhysRevLett.4.173

[37] Melngailis, I. ; Stillman, G. E. ; Dimmock, J. O. ; Wolfe, C. M.: Far-infrared recombination radiation from impact-ionized shallow donors in GaAs. In: Phys. Rev. Lett. 23 (1969), S. 1111-1114

[38] Hrostowski, H. J. ; Kaiser, R.H.: Infrared Absorption of Oxygen in Silicon. In: Phys. Rev. 107 (1957), Aug, Nr. 4, S. 966-972. http: //dx.doi.org/10.1103/PhysRev.107.966. - DOI 10.1103/PhysRev.107.966

[39] Larsen, D. M.: Shallow donor levels of InSb in a magnetic field. In: J. Phys. Chem. Solids 29 (1968), S. 271-280

[40] Eaves, L. ; Portal, J.C.: A review of the magneto-impurity effect in semiconductors. In: J. Phys. C 12 (1979), S. 2809-2825

[41] Pidgeon, C. R. ; Vaas, A. ; Allen, G. R. ; Prettl, W. ; Eaves, L.: Nonlinear Far-Infrared Magnetoabsorption and Optically Detected Magnetoimpurity Effect in n-GaAs. In: Phy. Rev. Lett. 50 (1983), S. 1309

[42] Allan, G. R. ; Black, A. ; Pidgeon, C. R. ; Gornik, E. ; SeidenBusch, W. ; Colter, P.: Impurity and Landau-level electron lifetime in n-type GaAs. In: Phys. Rev. B 31 (1985), S. 3560-3567 
[43] Planken, P. C. M. ; van Son, P. C. ; Hovenier, J. N. ; Klaassen, T. O. ; Wenckebach, W. T. ; Murdin, B. N. ; Knippels, G. M. H.: Far-infrared picosecond time-resolved measurement of the freeinduction decay in GaAs:Si. In: Phys. Rev. B 51 (1995), S. 9643-9647

[44] Cole, B. E. ; Williams, J. B. ; King, B. T. ; Sherwin, M. S. ; Stanley, C. R.: Coherent manipulation of semiconductor quantum bits with terahertz radiation. In: Nature (London) 410 (2001), S. 60-63

[45] Brandi, H. S. ; Latgé, A. ; Oliveira, L. E.: Rabi oscillations, coherent properties, and model qubits in two-level donor systems under terahertz radiation. In: Phys. Rev. B 68 (2003), Dec, Nr. 23, S. 233206. http://dx.doi.org/10.1103/PhysRevB.68.233206. - DOI 10.1103/PhysRevB.68.233206

[46] Doty, M. F. ; King, B. T. ; Sherwin, M. S. ; Stanley, C. R.: Verification of polarization selection rules and implementation of selective coherent manipulations of hydrogenic transitions in $n$-GaAs. In: Phys. Rev. B 71 (2005), S. 201201(R)

[47] Allen, D. G. ; Sherwin, M. S. ; Stanley, C. R.: Optically detected measurement of the ground-state population of an ensemble of neutral donors in GaAs. In: Physical Review B (Condensed Matter and Materials Physics) 72 (2005), Nr. 3, 035302. http://dx.doi.org/10. 1103/PhysRevB.72.035302. - DOI 10.1103/PhysRevB.72.035302

[48] Komiyama, S.: Far-Infrared Emission from Population-Inverted HotCarrier System in p-Ge. In: Phys. Rev. Lett. 48 (1982), Jan, Nr. 4, S. 271-274. http://dx.doi.org/10.1103/PhysRevLett.48.271. - DOI 10.1103/PhysRevLett.48.271

[49] S. Komiyama, N. I. ; Akasaka, Y.: Evidence for induced far-infrared emission from p-Ge in crossed electric and magnetic fields. In: AppPhys. Lett. 47 (1985), S. 958

[50] Unterrainer, K. ; Kremser, C. ; Gornik, E. ; Pidgeon, C. R. ; Ivanov, Y. L. ; Haller, E. E.: Tunable cyclotron-resonance laser in germanium. In: Phys. Rev. Lett. 64 (1990), May, Nr. 19, S. $2277-$ 2280. http://dx.doi.org/10.1103/PhysRevLett.64.2277. - DOI 10.1103/PhysRevLett.64.2277

[51] Pfeffer, P. ; Zawadzki, W. ; Unterrainer, K. ; Kremser, C. ; Wurzer, C. ; Gornik, E. ; Murdin, B. ; Pidgeon, C. R.: p-type 
Ge cyclotron-resonance laser: Theory and experiment. In: Phys. Rev. B 47 (1993), Feb, Nr. 8, S. 4522-4531. http://dx.doi.org/10.1103/ PhysRevB.47.4522. - DOI 10.1103/PhysRevB.47.4522

[52] Hovenier, J. N. ; Muravjov, A. V. ; Pavlov, S. G. ; Shastin, V. N. ; Strijbos, R. C. ; Th: Active mode locking of a p-Ge hot hole laser. In: Applied Physics Letters 71 (1997), Nr. 4, S. 443-445

[53] Muravjov, A. V. ; Withers, S. H. ; Strijbos, R. C. ; Pavlov, S. G. ; Shastin, V. N. ; Peale, R. E.: Actively mode-locked p-Ge laser in Faraday configuration. In: Applied Physics Letters 75 (1999), Nr. 19, S. 2882-2884

[54] Fredricksen, C. J. ; Nelson, E. W. ; Muravjov, A. V. ; Peale, R. E.: High field p-Ge laser operation in permanent magnet assemblies. In: Infrared Phys. \&6 Tech. 44 (2003), S. 79-84

[55] Hubers, H.-W. ; Pavlov, S. G. ; Shastin, V. N.: Terahertz lasers based on germanium and silicon. In: Semiconductor Science and Technology 20 (2005), Nr. 7, S. S211-S221

[56] Pavlov, S. G. ; Zhukavin, R. K. ; Orlova, E. E. ; Shastin, V. N. ; Kirsanov, A. V. ; Hübers, H.-W. ; Auen, K. ; Riemann, H.: Stimulated emission from donor transitions in silicon. In: Phys. Rev. Lett. 84 (2000), S. 5220-5223

[57] Shastin, V. N. ; Zhukavin, R. K. ; Orlova, E. E. ; Pavlov, S. G. ; Rummeli, M. H. ; Hubers, H. W. ; Hovenier, J. N. ; KlaAssen, T. O. ; Riemann, H. ; Bradley, I. V. ; Meer, A. F. G. d.: Stimulated terahertz emission from group-V donors in silicon under intracenter photoexcitation. In: Applied Physics Letters 80 (2002), Nr. 19

[58] Pavlov, S. G. ; Hubers, H. W. ; Hovenier, J. N. ; Klaassen, T. O. ; Carder, D. A. ; Phillips, P. J. ; Redlich, B. ; Riemann, H. ; Zhukavin, R. K. ; Shastin, V. N.: Stimulated terahertz stokes emission of silicon crystals doped with antimony donors. In: Physical Review Letters 96 (2006), Nr. 3, S. 4

[59] SchölL, E. (Hrsg.): Theory of Transport Properties of Semiconductor Nanostructures. London : Chapman \& Hall, 1998

[60] Mahan, G. D.: Many-Particle Physics. 3. New York : Kluwer, 2000 
[61] GLIESCHE, A.: Evolution of open many-electron systems: from a quantum statistical description towards the Semi-Classical Boltzmann Equation, Ecole Polytechnique Federale de Lausanne, Diss., 2007

[62] Schilp, J. ; Kuhn, T. ; Mahler, G.: Electron-phonon quantum kinetics in pulse-excited semiconductors: Memory and renormalization effects. In: Phys. Rev. B 50 (1994), Aug, Nr. 8, S. 5435-5447. http://dx.doi.org/10.1103/PhysRevB.50.5435. - DOI 10.1103/PhysRevB.50.5435

[63] Ashcroft, N. W. ; Mermin, N. D.: Solid State Physics. Brooks/Cole, 1976

[64] Haug, H. ; Koch, S. W.: Quantum Theory of the Optical and Electronic Properties of Semiconductors. Singapore: World Scientific, 1993

[65] Drude, P.: Zur Elektronentheorie I. In: Ann. Phys. (Leipzig) 1 (1900), S. 566-613

[66] Van Exter, M. ; Grischkowsky, D.: Carrier dynamics of electrons and holes in moderately doped silicon. In: Phys. Rev. B 41 (1990), S. $12140-12149$

[67] Katzenellenbogen, N. ; Grischkowsky, D.: Electrical characterization to $4 \mathrm{THz}$ of $n$ - and $p$-type GaAs using $\mathrm{THz}$ time-domain spectroscopy. In: Appl. Phys. Lett. 61 (1992), S. 840-842

[68] Spitzer, W. G. ; Whelan, J. M.: Infrared absorption and electron effective mass in $n$-type gallium arsenide. In: Phys. Rev. 114 (1959), S. 59-63

[69] Dumke, W. P.: Quantum Theory of Free Carrier Absorption. In: Phys. Rev. 124 (1961), Dec, Nr. 6, S. 1813-1817. http://dx.doi. org/10.1103/PhysRev.124.1813. - DOI 10.1103/PhysRev.124.1813

[70] Myciblski, J. ; Aziza, A. ; Mycielski, A. ; Balkanski, M.: Theory of free-carrier absorption in polar semiconductors due to electron-LOphonon interaction. In: physica status solidi (b) 67 (1975), Nr. 2, S. $447-454$

[71] Jensen, B.: Multiphoton free-carrier absorption at high intensities in polar semiconductors. In: Phys. Rev. B 24 (1981), Nov, Nr. 10, S. 5932-5948. http://dx.doi.org/10.1103/PhysRevB.24.5932. - DOI 10.1103/PhysRevB.24.5932 
[72] Fletcher, K. ; Butcher, P. N.: An exact solution of the linearized Boltzmann equation with applications to the Hall mobility and the Hall factor of n-GaAs. In: J. Phys. C 5 (1972), S. 212-224

[73] Stillmann, G. E. ; Wolfe, C. M. ; Dimmock, J. O.: Hall coefficient factor for polar mode scattering in n-type GaAs. In: J. Phys. Chem. Solids 31 (1970), S. 1199-1204

[74] Bardeen, J. ; Shockley, W.: Deformation potentials and mobilities in non-polar crystals. In: Phys. Rev. 80 (1950), S. 72-80

[75] HüBneR, K.: Piezoelectricity in zincblende- and wurtzite-type crystals. In: Phys. Stat. Sol. B 57 (1973), S. 241-243

[76] Pötz, W. ; VogL, P.: Theory of optical-phonon deformation potentials in tetrahedral semiconductors. In: Phys. Rev. B 24 (1981), Aug, Nr. 4, S. 2025-2037. http://dx.doi.org/10.1103/PhysRevB. 24.2025. - DOI 10.1103/PhysRevB.24.2025

[77] Anastassakis, E. ; Cardona, M.: Internal strains and Raman-active optical phonons. In: Phys. Stat. Sol 104 (1981), S. 589-600

[78] Ruch, J. G. ; Kino, G. S.: Measurement of the velosity-field characteristic of gallim arsenide. In: Applied Physics Letters 10 (1967), Nr. 2, S. $40-42$

[79] Butcher, P. N. ; Fawcett, W.: Calculation of the velocity-field characteristic for gallium arsenide. In: Phys. Lett. 21 (1966), S. 489490

[80] Ridley, B. K. ; Watkins, T. B.: The possibillity of a negative resistance effect in semiconductors. In: PROC. Phys. Soc. 78 (1961), S. 293

[81] Shah, J. ; Deveaud, Benoit ; Damen, T.C. ; Tsang, W.T. ; GosSARD, A.C. ; Lugli, P.: Determination of Intervally Scattering Rates in GaAs by Subpicosecond Luminescence Spectroscopy. In: Phy. Rev. Lett. 59 (1987), S. 2222

[82] Kim, D.-S. ; YU, P. Y.: Hot-electron relaxations and hot phonons in GaAs studied by subpicosecond Raman scattering. In: Phys. Rev. B 43 (1991), S. 4158-4169 
[83] Leitenstorfer, A. ; Hunsche, S. ; Shah, J. ; Nuss, M. C. ; Knox, W. H.: Femtosecond high-field transport in compound semiconductors. In: Phys. Rev. B 61 (2000), S. 16642-16652

[84] Gunn, J. B.: Microwave oscillations of current in III-V semicunductors. In: Solid State Comm. 1 (1963), S. 88-91

[85] Ridley, B. K.: Quantum Processes in Semiconductors. 3. Oxford : Oxford University Press, 1993

[86] Landau, L. D. ; Lifschitz, E. M.: Lehrbuch der Theoretischen Physik VIII - Elektrodynamik der Kontinua. 7. Berlin : Akademie-Verlag, 1985

[87] Wegener, M.: Quantum coherence in semiconductors. In: J. Lumin. 87 (2000), S. 20-24

[88] Fürst, C. ; Leitenstorfer, A. ; Laubereau, A. ; Zimmermann, R.: Quantum Kinetic Electron-Phonon Interaction in GaAs: Energy Nonconserving Scattering Events and Memory Effects. In: Phys. Rev. Lett. 78 (1997), May, Nr. 19, S. 3733-3736. http://dx.doi.org/10. 1103/PhysRevLett.78.3733. - DOI 10.1103/PhysRevLett.78.3733

[89] Aihara, M.: Non-Markovian theory of nonlinear-optical phenomena associated with the extremely fast relaxation in condensed matter. In: Phys. Rev. B 25 (1982), Jan, Nr. 1, S. 53-60. http://dx.doi.org/ 10.1103/PhysRevB.25.53. - DOI 10.1103/PhysRevB.25.53

[90] Nibbering, E. T. J. ; Wiersma, D. A. ; Duppen, K.: Femtosecond non-Markovian optical dynamics in solution. In: Phys. Rev. Lett. 66 (1991), May, Nr. 19, S. 2464-2467. http://dx.doi.org/10.1103/ PhysRevLett.66.2464. - DOI 10.1103/PhysRevLett.66.2464

[91] Bányai, L. ; Tran Thoai, D. B. ; Reitsamer, E. ; Haug, H. ; Steinbach, D. ; Wehner, M. U. ; Wegener, M. ; Marschner, T. ; Stolz, W.: Exciton-LO-phonon quantum kinetics: Evidence of memory effects in bulk GaAs. In: Phys. Rev. Lett. 75 (1995), S. 2188-2191

[92] Bar-Ad, S. ; Kner, P. ; Marquezini, M. V. ; Chemla, D. S. ; EL SAYED, K.: Carrier Dynamics in the Quantum Kinetic Regime. In: Phys. Rev. Lett. 77 (1996), S. 3177-3180

[93] Leitenstorfer, A. ; Fürst, C. ; Laubereau, A. ; Kaiser, W. ; Tränkle, G. ; Weimann, G.: Femtosecond carrier dynamics in GaAs 
far from equilibrium. In: Phys. Rev. Lett. 76 (1996), S. 1545-1548. - ; 76, 3662(E) (1996)

[94] Wehner, M. U. ; Chemla, D. S. ; Wegener, M.: Electron-LOphonon quantum kinetics in semiconductor quantum wells. In: Phys. Rev. B 58 (1998), S. 3590-3593

[95] Steinbach, D. ; Kocherscheidt, G. ; Wehner, M. U. ; Kalt, H. ; Wegener, M. ; OhKawa, K. ; Hommel, D. ; Axt, V. M.: Electronphonon quantum kinetics in the strong-coupling regime. In: Phys. Rev. B 60 (1999), Nov, Nr. 17, S. 12079-12090. http://dx.doi.org/10. 1103/PhysRevB.60.12079. - DOI 10.1103/PhysRevB.60.12079

[96] Woggon, U. ; Gindele, F. ; Langbein, W. ; Hvam, J. M.: Quantum kinetic exciton-LO-phonon interaction in CdSe. In: Phys. Rev. B 61 (2000), S. 1935-1940

[97] Wehner, M. U. ; Ulm, M. H. ; Chemla, D. S. ; Wegener, M.: Coherent control of electron-LO-phonon scattering in bulk GaAs. In: Phys. Rev. Lett. 80 (1998), S. 1992-1995

[98] Ogawa, Y. ; Minami, F.: Coherent control of electron-phonon scattering and the dephasing process in GaAs multiple quantum wells. In: Physical Review B (Condensed Matter and Materials Physics) 75 (2007), Nr. 7, 073302. http://dx.doi.org/10.1103/PhysRevB.75. 073302. - DOI 10.1103/PhysRevB.75.073302

[99] Mandelung, O.: Festkörpertheorie II. 1. Springer, 1972

[100] Leitenstorfer, A. ; Hunsche, S. ; Shah, J. ; Nuss, M. C. ; Knox, W. H.: Femtosecond charge transport in polar semiconductors. In: Phys. Rev. Lett. 82 (1999), S. 5140-5143

[101] Cho, G. C. ; Küтt, W. ; Kurz, H.: Subpicosecond time-resolved coherent-phonon oscillations in GaAs. In: Phys. Rev. Lett. 65 (1990), S. $764-766$

[102] Kütt, W. ; Cho, G. C. ; Pfeiffer, T. ; Kurz, H.: Subpicosecond generation and decay of coherent phonons in III-V compounds. In: Semicond. Sci. Tech. 7 (1992), S. B77

[103] Bargheer, M. ; Zhavoronkov, N. ; Gritsai, Y. ; Woo, J. C. ; Kim, D. S. ; Woerner, M. ; Elsaesser, T.: Coherent atomic motions in a nanostructure studied by femtosecond x-ray diffraction. In: Science 306 (2004), S. 1771-1773 
[104] Huber, R. ; Tauser, F. ; Brodschelm, A. ; Bichler, M. ; ABStreiter, G. ; Leitenstorfer, A.: How many-particle interactions develop after ultrafast excitation of an electron-hole plasma. In: Nature (London) 414 (2001), S. 286-289

[105] Hase, M. ; Kitajima, M. ; Constantinescu, A. M. ; Petek, H.: The birth of a quasiparticle in silicon observed in time-frequency space. In: Nature (London) 426 (2003), S. 51-54

[106] FrÖHLICH, H.: Elctrons in lattice fields. In: Adv. Phys. 3 (1954), S. 325

[107] Peeters, F. M. ; Devreese, J. T.: Radius, self-induced potential, and number of virtual optical phonons of a polaron. In: Phys. Rev. B 31 (1985), S. $4890-4899$

[108] Pfeifer, T. ; Dekorsy, T. ; Kimtt, W. ; Kurz, H.: Generation Mechanism for Coherent LO Phonons in Surface-Space-Charge Fields of III-V-Compounds. In: Appl. Phys. A 55 (1992), S. 482-488

[109] Vallée, F. ; Bogani, F.: Coherent time-resolved investigation of LOphonon dynamics in GaAs. In: Phys. Rev. B 43 (1991), S. 12049-12052

[110] Planken, P. C. M. ; Nuss, M. C. ; Brener, I. ; Goosen, K. W. ; Luo, M. S. C. ; Chuang, S. L. ; Pfeiffer, L.: Terahertz emission in single quantum wells after coherent optical excitation of light hole and heavy hole excitons. In: Phys. Rev. Lett. 69 (1992), S. 3800-3803

[111] Roskos, H. G. ; Nuss, M. C. ; Shah, J. ; Leo, K. ; Miller, D. A. B. ; Fox, A. M. ; Schmitt-Rink, S. ; Köhler, K.: Coherent submillimeter-wave emissions from charge oscillations in a double-well potential. In: Phys. Rev. Lett. 68 (1992), S. 2216-2219

[112] Waschke, C. ; Roskos, H. G. ; Schwedler, R. ; Leo, K. ; Kurz, H. ; KÖHLER, K.: Coherent submillimeter-wave emission from Bloch oscillations in a semiconductor superlattice. In: Phys. Rev. Lett. 70 (1993), S. 3319-3322

[113] Mooradian, A. ; Wright, G. B.: Observation of the Interaction of Plasmons with Longitudinal Optical Phonons in GaAs. In: Phys. Rev. Lett. 16 (1966), May, Nr. 22, S. 999. http://dx.doi.org/10.1103/ PhysRevLett.16.999. - DOI 10.1103/PhysRevLett.16.999 
[114] El Sayed, K. ; Schuster, S. ; Haug, H. ; Herzel, F. ; HenNEBERGER, K.: Subpicosecond plasmon response: Buildup of screening. In: Phys. Rev. B 49 (1994), Mar, Nr. 11, S. 7337-7344. http: //dx.doi.org/10.1103/PhysRevB.49.7337. - DOI 10.1103/PhysRevB.49.7337

[115] Vu, Q. T. ; Haug, H. ; Hügel, W. A. ; Chatterjee, S. ; Wegener, M.: Signature of Electron-Plasmon Quantum Kinetics in GaAs. In: Phys. Rev. Lett. 85 (2000), Oct, Nr. 16, S. 35083511. http://dx.doi.org/10.1103/PhysRevLett.85.3508. - DOI 10.1103/PhysRevLett.85.3508

[116] Huber, R. ; Kübler, C. ; Tübel, S. ; Leitenstorfer, A. ; Vu, Q. T. ; Haug, H. ; Köhler, F. ; Amann, M.-C.: Femtosecond Formation of Coupled Phonon-Plasmon Modes in InP: Ultrabroadband THz Experiment and Quantum Kinetic Theory. In: Physical Review Letters 94 (2005), Nr. 2, S. 027401. http://dx.doi.org/10.1103/ PhysRevLett.94.027401. - DOI 10.1103/PhysRevLett.94.027401

[117] Haug, H.: Interband Quantum Kinetics with LO-Phonon Scattering in a Laser-Pulse-Excited Semiconductor I. Theory. In: Phys. Stat. Sol. B 177 (1992), S. 139-148

[118] Bányai, L. ; Thoai, D.B. T. ; Remling, C. ; Haug, H.: Interband Quantum Kinetics with LO-Phonon Scattering in a Laser-PulseExcited Semiconductor II. Numerical Studies. In: Phys. Stat. Sol. B 177 (1992), S. 149-157

[119] Zimmermann, R.: Carrier kinetics for ultrafast optical pulses. In: J. Lumin. 53 (1992), S. 187-190

[120] Tran Thoai, D. B. ; Haug, H.: Band-edge quantum kinetics for coherent ultrashort-pulse spectroscopy in polar semiconductors. In: Phys. Rev. B 47 (1993), Feb, Nr. 7, S. 3574-3581. http://dx.doi. org/10.1103/PhysRevB.47.3574. - DOI 10.1103/PhysRevB.47.3574

[121] Bányai, L. ; Thoai, D. B. T. ; Remling, C. ; Haug, H.: Coherent interband effects in quantum kinetics. In: Phys. Stat. Sol. B 188 (1995), S. 387

[122] Fürst, C. ; Leitenstorfer, A. ; Laubereau, A. ; Zimmermann, R.: Quantum kinetic electron-phonon interaction in GaAs: Energy nonconserving scattering events and memory effects. In: Phys. Rev. Lett. 78 (1997), S. 3733-3736 
[123] Warren, W. S. ; Rabitz, H. ; Dahleh, M.: Coherent Control of Quantum Dynamics: The Dream Is Alive. In: Science 259 (1993), S. $1581-1589$

[124] Rabitz, H. ; Vivie-Riedle, R. ; Motzkus, M. ; Kompa, K.: Whither the Future of Controling Quantum Phenomena. In: Science 288 (2000), S. 824-828

[125] Wehner, M. U. ; Ulm, H. C. ; Wegener, M.: Scanning interferometer stabilized by use of Pancharatnams phase. In: Opt. Lett. 22 (1997), S. $1455-1457$

[126] Herbst, M. ; Glanemann, M. ; Axt, V. M. ; Kuhn, T.: Electronphonon quantum kinetics for spatially inhomogeneous excitations. In: Phys. Rev. B 67 (2003), May, Nr. 19, S. 195305. http://dx.doi.org/ 10.1103/PhysRevB.67.195305. - DOI 10.1103/PhysRevB.67.195305

[127] Elsaesser, T.: Femtosecond mid-infrared spectroscopy of low-energy excitations in solids. In: Appl. Phys. A 79 (2004), S. 1627-1634

[128] Koeberg, M. ; Hendry, E. ; Schins, J. M. ; Laarhoven, H. A. ; Flipse, C. F. J. ; Reimann, K. ; Woerner, M. ; Elsaesser, T. ; Bonn, M.: Simultaneous ultrafast probing of intramolecular vibrations and photoinduced charge carriers in rubrene using broadband timedomain THz spectroscopy. In: Phys. Rev. B 75 (2007), S. 195216-1-5

[129] Chan, W. L. ; Deibel, J. ; Mittelmann, D.M.: Imaging with THz radiation. In: Rep. Prog. Phys. 70 (2007), S. 1325

[130] Rosner, B. T. ; Weide, D. B.: High-Frequency near-field microscopy. In: Rev. Sci. Instrum. 73 (2002), S. 2505-2525

[131] C. Spielmann, T. B. P.F. Curley C. P.F. Curley ; Krausz, F.: Ultrabroadband femtosecond lasers. In: IEEE J. Quantum Electron 30 (1994), S. 1100-1114

[132] Koecher, W.: Solid-State Laser Engeneering. 5. Springer, 1999

[133] Matsubara, E. ; Yamane, K. ; Sekikawa, T. ; Yamashita, M.: Generation of 2.6 fs optical pulses using induced-phase modulation in a gas-filled hollow fiber. In: J. opt. Soc. Am. B 24 (2007), S. 958-989

[134] Binhammer, T. ; Rittweger, E. ; Morgner, U. ; Ell, R. ; KärtNER, F. X.: Spectral phase control and tmeporal superresolution toward the single-cycle pulse. In: Opt. Lett 31 (2006), S. 1552-1554 
[135] Skupin, S. ; Stibenz, G. ; Berge, L. ; Lederer, F. ; Sokollik, T. ; Schnurer, M. ; Zhavoronkov, N. ; Steinmeyer, G.: Selfcompression by femtosecond pulse filamentation: Experiments versus numerical simulations. In: Physical Review E (Statistical, Nonlinear, and Soft Matter Physics) 74 (2006), Nr. 5, S. 056604

[136] M. Hentschel, F. K. Z. Cheng C. Z. Cheng ; Spielmann, C.: Generation of 0.1-TW optical pulses with a single-stage Ti:Sapphire amplifier at a 1-kHz repetition rate. In: Appl. Phys. B 70 (2000), S. S161-S164

[137] Pockels, F.: Ueber den Einfluss des elektrostatischen Feldes auf das optische Verhalten piëzoelektrischer Krystalle. In: Abh. Ges. Wissensch. Göttingen 39 (1894), S. 1-204

[138] Reimann, K.: Table-top sources of ultrashort THz pulses. In: Rep. Prog. Phys. 70 (2007), S. 1597-1632

[139] Zhong, H. ; Karpowicz, N. ; Zhang, X.-C.: Terahertz emission profile from laser-induced air plasma. In: Appl. Phys. Lett. 88 (2006), S. 261103

[140] Hattori, T. ; Rundsawang, R. ; Ohta, K. ; Tukamoto, K.: Gaussian beam analysis of temporal waveform of focused terahertz pulses. In: Jpn. J. Appl. Phys. 41 (2002), S. 5198-5204

[141] Lindner, F. ; Paulus, G. G. ; Walther, H. ; Baltruska, A ; Goulielmakis, E ; Lezius, M. ; Krausz, F.: Gouy Phase Shift for Few-Cycle Laser Pulses. In: Phys. Rev. Lett. 92 (2004), S. 113001

[142] Feng, S. ; Winful, H. G.: Physical origin of the Gouy phase shift. In: Opt. Lett. 26 (2001), S. 485-487

[143] Hunsche, S. ; Feng, S. ; Winful, H. G. ; Leitenstorfer, A. ; Nuss, M. C. ; IPpen, E. P.: Spatiotemporal focusing of single-cycle light pulses. In: J. Opt. Soc. Am. A 16 (1999), S. 2025-2028

[144] Feng, S. ; Winful, H. G. ; Hellwarth, R. W.: Gouy shift and temporal reshaping of focused single-cycle electromagnetic pulses. In: Opt. Lett. 23 (1998), S. 385-387. - ; 23, 1141(E) (1998)

[145] Faust, W. L. ; Henry, C. H.: Mixing of Visible and Near-Resonance Infrared Light in GaP. In: Phys. Rev. Lett. 17 (1966), S. 1265 
[146] Leitenstorfer, A. ; Hunsche, S. ; Shah, J. ; Nuss, M. C. ; Knox, W. H.: Detectors and sources for ultrabroadband electro-optic sampling: Experiment and theory. In: Appl. Phys. Lett. 74 (1999), S. $1516-1518$

[147] Wu, Q. ; Zhang, X.-C.: 7 terahertz broadband GaP electro-optic sensor. In: Appl. Phys. Lett. 70 (1997), S. 1784-1786

[148] DAI, J. ; XIE, X. ; Zhang, X.-C.: Detection of broadband terahertz waves with a laser-induced plasma in gases. In: Phys. Rev. Lett. 97 (2006), S. 103903

[149] Mclaughlin, C. V. ; Zheng, X. ; Hayden, L. M.: Comparison of parallel-plate and in-plane polymer films for terahertz sensing. In: Appl. Opt. 46 (2007), S. 6283-6289

[150] Zheng, X. ; Mclaughlin, C. V. ; Cunningham, P. ; Hayden, L. M.: Organic Broadband Terahertz Sources and Sensors. In: J. Nanoelectron. Optoelectron. 2 (2007), S. 58-66

[151] Chen, C.-W. ; Hsu, Y.-K. ; Huang, J. Y. ; Chang, C.-S. ; Zhang, J.-Y.; PAN, C.-L.: Generation properties of coherent infrared radiation in the optical absorption region of GaSe crystal. In: Opt. Express 14 (2006), S. 10636-10644

[152] Dimiriev, V. G. ; Gurzadyan, G. G. ; Nikogosyan, D. N.: Handbook of nonlinear optical crystals, Springer series in optical science, VOL. 64. Springer, 1997

[153] Kaindl, R. A. ; Smith, D. C. ; Joschko, M. ; Hasselbeck, M. P. ; Woerner, M. ; Elsaesser, T.: Femtosecond infrared pulses tunable from 9 to $18 \mu \mathrm{m}$ at an 88-MHz repetition rate. In: Opt. Lett. 23 (1998), S. $861-863$

[154] Reimann, K. ; Smith, R. P. ; Weiner, A. M. ; Elsaesser, T. ; Woerner, M.: Direct field-resolved detection of terahertz transients with amplitudes of megavolts per centimeter. In: Opt. Lett. 28 (2003), S. $471-473$

[155] Vodopyanov, K. L. ; Voevodin, V. G.: $2.8 \mu \mathrm{m}$ laser pumped type I and type II travelling-wave optical parametric generator in GaSe. In: Opt. Commun. 114 (1995), S. 333-335 
[156] Bartal, B. ; Kozma, I. Z. ; Stepanov, A. G. ; Almási, G. ; Kuhl, J. ; Riedle, E. ; Hebling, J.: Toward generation of $\mu \mathrm{J}$ range sub-ps THz pulses by optical rectification. In: Appl. Phys. B 86 (2007), S. 419-423

[157] Hoffmann, M. C. ; Yeh, K. L. ; J, J. H. ; Nelson, K. A.: Efficient terahertz generation by optical rectification at $1035 \mathrm{~nm}$. In: Opt. Express 15 (2007), S. 11706-1171

[158] Stepanov, A. G. ; Kuhl, J. ; Kozma, I. Z. ; Riedle, E. ; Almási, G. ; Hebling, J.: Scaling up the energy of $\mathrm{THz}$ pulses created by optical rectification. In: Opt. Express 13 (2005), S. 5762-5768

[159] Hebling, J. ; Stepanov, A.G. ; Almasi, G. ; Bartal, B. ; Kuhl, J.: Tunable $\mathrm{THz}$ pulse generation by optical rectification of ultrashort laser pulses with tilted pulse fronts. In: Appl. Phys. B 78 (2004), S. $593-599$

[160] A. Schneider, P. G. I. Biaggio B. I. Biaggio: Optimized generation of $\mathrm{THz}$ pulses via optical rectification in the organic salt DAST. In: Opt. Comm. 224 (2003), S. 337-341

[161] Hamster, H. ; Falcone, R. W.: Proposed Source of Sub-picosecond Far Infrared Radiation. Springer, 1990

[162] Hamster, H. ; Sullivan, A. ; Gordon, S. ; White, W. ; FalCONE, R. W.: Subpicosecond, electromagnetic pulses from intense laser-plasma interaction. In: Phys. Rev. Lett. 71 (1993), Oct, Nr. 17, S. 2725-2728. http://dx.doi.org/10.1103/PhysRevLett.71.2725. - DOI 10.1103/PhysRevLett.71.2725

[163] Cook, D. J. ; Hochstrasser, R. M.: Intense terahertz pulses by four-wave rectification in air. In: Opt. Lett. 25 (2000), S. 1210-1212

[164] Corwin, J. P. ; Tsekouras, A. A. ; Iedema, M. J. ; Wu, K. ; Ellison, G. B.: Immobility of protons in ice from 30 to 190 K. In: Nature 398 (1999), S. 405-407

[165] Nahata, A. ; Heinz, T. F.: Detection of freely propagating terahertz radiation by use of optical second-harmonic generation. In: Opt. Lett 23 (1998), S. 67-69

[166] Kress, M. ; Löffler, T. ; Eden, S. ; Thomson, M. ; Roskos, H. G.: Terahertz-pulse generation by photoionization of air with laser 
pulses composed of both fundamental and second-harmonic waves. In: Opt. Lett. 29 (2004), S. 1120-1122

[167] Kim, K. Y. ; Glownia, J. H. ; Taylor, A. J. ; Rodriguez, G.: Terahertz emission from ultrafast ionizing air in symmetry-broken laser fields. In: Opt. Express 15 (2007), S. 4577

[168] Côté, D. ; Fraser, J. M. ; DeCamp, M. ; Bucksbaum, P. H. ; van DrIEL, H. M.: THz emission from coherently controlled photocurrents in GaAs. In: Appl. Phys. Lett. 75 (1999), S. 3959-3961

[169] Dupont, E. ; Corkum, P. B. ; Liu, H. C. ; Buchanan, M. ; Wasilewski, Z. R.: Phase-Controlled Currents in Semiconductors. In: Phys. Rev. Lett. 74 (1995), May, Nr. 18, S. 3596-3599. http:// dx.doi.org/10.1103/PhysRevLett.74.3596. - DOI 10.1103/PhysRevLett.74.3596

[170] Mlejnek, M. ; Wright, E. M. ; Moloney, J. V.: Femtosecond pulse propagation in argon: A pressure dependence study. In: Phys. Rev. E 58 (1998), Oct, Nr. 4, S. 4903-4910. http://dx.doi.org/10. 1103/PhysRevE.58.4903. - DOI 10.1103/PhysRevE.58.4903

[171] Aktturk, S. ; D’Amico, C. ; Franco, M. ; Couairon, A. ; MysyROWICZ, A.: Pulse shortening, spatial mode cleaning, and intense terahertz generation by filamentation in xenon. In: Phys. Rev. A 76 (2007), S. 063819

[172] Karpowicz, N. ; Dai, J. ; Lu, X. ; Chen, Y. ; Yamaguchi, M. ; Zhao, H. ; Zhang, X.-C. ; Zhang, L. ; Zhang, C. ; PriceGallagher, M. ; Fletcher, C. ; Mamer, O. ; Lesimple, A. ; Johnson, K.: Coherent heterodyne time-domain spectroscopy covering the entire terahertz gap. In: Appl. Phys. Lett. 93 (2008), S. 011131

[173] Fusi, T. ; Suzuki, T.: Generation of sub-two-cycle mid-infrared pulses by four-wave mixing through filamentation in air. In: Opt. Lett. 32 (2007), S. 3330-3332

[174] XIE, Xu ; DaI, J. ; Zhang, X.-C.: Coherent control of THz wave generation in ambient air. In: Phys. Rev. Lett. 96 (2006), S. 075005

[175] LÖFfleR, T.: Erzeugung intensiver Pulse im Terahertzfrequenzbereich mittels laser-generierter Plasmen, Universität Frankfurt am Main, Diss., 2003 
[176] Thomson, M. D. ; Kress, M. ; Löffler, T. ; Roskos, H. G.: Broadband $\mathrm{THz}$ emission from gas plasmas induced by femtosecond optical pulses: From fundamentals to application. In: Laser \& Photon. Rev. 1 (2007), S. 349-368

[177] Bartel, T. ; Gaal, P. ; Reimann, K. ; Woerner, M. ; Elsaesser, T.: Generation of single-cycle $\mathrm{THz}$ transients with high electric-field amplitudes. In: Opt. Lett. 30 (2005), S. 2805-2807

[178] Shih, T. ; Reimann, K. ; Woerner, M. ; Elsaesser, T. ; Waldmüller, I. ; Knorr, A. ; Hey, R. ; Ploog, K. H.: Nonlinear response of radiatively coupled intersubband transitions of quasi-twodimensional electrons. In: Phys. Rev. B 72 (2005), S. 195338-1-8

[179] Stroucken, T. ; Knorr, A. ; Thomas, P. ; Koch, S. W.: Coherent dynamics of radiatively coupled quantum-well excitons. In: Phys. Rev. B 53 (1996), S. 2026-2033

[180] Pollard, W. T. ; Mathies, R. A.: Analysis of femtosecond dynamic absorption spectra of nonstationary states. In: Annu. Rev. Phys. Chem. 43 (1992), S. 497-523

[181] Chachisvilis, M. ; Fidder, H. ; Sundström, V.: Electronic coherence in pseudo two-colour pump-probe spectroscopy. In: Chem. Phys. Lett. 234 (1995), S. 141-150

[182] LePore, J. J.: An improved technique for selective etching of GaAs and $\mathrm{Ga}_{1-x} \mathrm{Al}_{x}$ As. In: J. Appl. Phys. 51 (1980), S. 6441

[183] Knigge, S.: private communication. (2007)

[184] Ruf, T. ; Cardona, M. ; Pickles, C. S. J. ; Sussmann, R.: Temperature dependence of the refractive index of diamond up to $925 \mathrm{~K}$. In: Phys. Rev. B 62 (2000), S. 16578-16581

[185] Fu, K.-M. C. ; Santori, C. ; Stanley, C. ; Holland, M. C. ; Yамамото, Y.: Coherent population trapping of electron spins in a high-purity $n$-type GaAs semiconductor. In: Phys. Rev. Lett. 95 (2005), S. 187405

[186] Hughes, S.: Breakdown of the area theorem: Carrier-wave Rabi flopping of femtosecond optical pulses. In: Phys. Rev. Lett. 81 (1998), S. 3363-3366 
[187] Mücke, O. D. ; Tritschler, T. ; Wegener, M. ; Morgner, U. ; KÄrTner, F. X.: Signatures of carrier-wave Rabi flopping in GaAs. In: Phys. Rev. Lett. 87 (2001), S. 057401-1-4

[188] Ziolkowski, R. W. ; Arnold, J. M. ; Gogny, D. M.: Ultrafast pulse interactions with two-level atoms. In: Phys. Rev. A 52 (1995), S. 3082-3094

[189] Mücke, O. D. ; Tritschler, T. ; Wegener, M. ; Morgner, U. ; KäRTNER, F. X.: Role of the carrier-envelope offset phase of few-cycle pulses in nonperturbative resonant nonlinear optics. In: Phys. Rev. Lett. 89 (2002), S. 127401

[190] Udem, T.: Condensed-matter physics: Rabi flopping sees the light. In: Nature (London) 420 (2002), S. 469-472

[191] Barnett, R. N. ; Cleveland, C. L. ; Joy, A. ; Landman, U. ; Schuster, G.B.: Charge Migration in DNA: Ion-Gated Transport. In: Science 294 (2001), S. 567-571

[192] Li, B. ; Zhao, J. ; Onda, K. ; Jordan, K. D. ; Yang, J. ; Petek, H.: Ultrafast Interfacial Proton-Coupled Electron Transfer. In: Science 311 (2006), S. 1436-1440

[193] Braun, M. ; Korff, C. ; Kiel, M. ; Zhavoronkov, N. ; Dreyer, J. ; Bargheer, M. ; Elsaesser, T. ; Root, C. ; Schrader, T.E ; Gilch, P. ; Zinth, W. ; Woerner, M.: Ultrafast Changes of Molecular Crystal Structure Induced by Dipole Solvation. In: Phys. Rev. Lett. 98 (2007), S. 248301

[194] Huber, R. ; Tauser, F. ; Brodschelm, A. ; Bichler, M. ; AbStreiter, G. ; Leitenstorfer, A.: How many-particle interactions develop after ultrafast excitation of an electron-hole plasma. In: Nature 414 (2001), S. 286-289

[195] Hase, M. ; Kitajima, M. ; Constantinescu, A.M. ; Petek, H.: The birth of a quasiparticle in silicon observed in time-frequency space. In: Nature 426 (2003), S. 51-54

[196] Rannow, H. M. ; Ch, A. ; Aeppli, G. ; Kimura, T. ; Tokura, Y.: Polarons and confinement of electronic motion to two dimensions in a layered manganite. In: Nature 440 (2006), S. 1025-1028 
[197] Bingham, R.: On the crest of a wake. In: Nature 445 (2007), S. $72-722$

[198] AL., I. B.: Energy doubling of $42 \mathrm{GeV}$ electrons in a metre-scale plasma wakefield accelerator. In: Nature 445 (2007), S. 741-744

[199] Magnus, W. ; Schoenmaker, W.: Dissipative motion of an electronphonon system in a uniform electric field: An exact solution. In: Phys. Rev. B 47 (1993), S. 1276-1281

[200] Magnus, W. ; Schoenmaker, W.: Dissipative motion of an electronphonon system in a uniform electric field: An exact solution. In: Phy. Rev. B 47 (1993), S. 1276-1281

[201] Press, W. H. ; Flannery, B. P. ; Teukolsky, S. A. ; Vetterling, W. T.: Numerical Recipes. Cambridge : Cambridge University Press, 1986

[202] Averitt, R.: Quasiparticles undressed. In: Nature Physics 4 (2008), S. $14-15$

[203] Jackson, J. D.: Klassische Elektrodynamik. 3. de Gruyter, 2002

[204] Gaal, P. ; Raschke, M.B. ; Reimann, K. ; Woerner, M.: Measuring optical frequencies in the $0-40 \mathrm{THz}$ range with non-synchronized electro-optic sampling. In: Nature Photonics 1 (2007), S. 577-580

[205] Leitensdorfer, A. ; Fürst, C. ; Laubereau, A.: Widely tunable Ti:sapphire laser with pulse jitter of less than 2 fs. In: Opt. Lett. 20 (1995), S. 916-918

[206] Ramond, T. M. ; Diddams, S. A. ; Hollberg, L. ; Bartels, A.: Phase-coherent link from optical to microwave frequencies by means of the broadband continuum from a 1-GHz Ti:sapphire femtosecond oscillator. In: Opt. Lett. 27 (2002), S. 1842-1844 


\section{Danksagung}

Mein besonderer Dank gilt Herrn Prof. Dr. Thomas Elsaesser für die Aufnahme an das Max-Born-Institut und die Betreuung dieser Arbeit.

Prof. Benson und Prof. Unterrainer danke ich für die spontane Bereitschaft die Gutachten anzufertigen.

Besonders bedanken möchte ich mich bei Dr. Michael Wörner für die Betreung dieser Arbeit. Er hat mit Begeisterung und vielen Ideen wesentlich zu ihrem Gelingen beigetragen.

Gleiches gilt für Prof. Dr. Klaus Reimann. Ihm gebührt zusätzlicher Dank für die gründliche Einarbeitung in das THz-Labor und die uneigennützige Hilfe bei allen experimentellen Fragen sowie allen Problemen des Alltags.

Wilhelm Kühn danke ich für die gute Zusammenarbeit und für das engagierte Anfertigen seiner Diplomarbeit in unserer Arbeitsgruppe.

Darüber hinaus bedanke ich mich bei

- Dr. Rudolf Hey für das Wachsen exzellenter Halbleiterproben, die noch nach Jahren neue Geheimnisse preisgeben.

- Satoshi Ashihara, Jens Bethge, Jong Seok Lee, Dr. Ulrich Schade, Dr. Günter Steinmeyer and Wolfgang Partz für die angenehme und engagierte Zusammenarbeit in verschiedenen Projekten.

- Frau Gabriele Kordaß für die tatkräftige Unterstützung bei technischen und administrativen belangen, sowie für das gewissenhafte Einschalten meiner Laser jeden Morgen zu früher Stunde.

- den Mitarbeiterinnen und Mitarbeitern der Werkstatt des MBI für die zuverlässige und unkomplizierte Unterstützung.

- Cynthia Aku-Leh, Zunaira Ansari, Matthias Bargheer, Markus Breusing, Anders Harpoeth, Clemens von Korff-Schmiesing, Claus Ropers, Phillip Rothhardt, Flavio Zamponi und allen nicht namentlich genannten Mitgliedern des Bereichs C für die angenehme Arbeitsatmosphäre während der letzten Jahre. 


\section{Selbständigkeitserklärung}

Hiermit erkläre ich, die vorliegende Dissertation selbstständig und ohne unerlaubte Hilfe angefertigt zu haben.

Ich erkläre die Kenntnisnahme der dem Verfahren zugrundeliegenden Promotionsordnung der Mathematisch-Naturwissenschaftlichen Fakultät I der Humboldt-Universität zu Berlin. 